\title{
Gibbs Measures for Models on Lines and Trees
}

\author{
Eric Ossami Endo
}

TESE APRESENTADA

$\mathrm{AO}$

Instituto De Matemática e Estatística

DA

UNIVERSIDADE DE SÃO PAULO

PARA

OBTENÇÃO DO TÍTUlO

$\mathrm{DE}$

DOUTOR EM CIÊNCIAS

\author{
Programa: Matemática Aplicada \\ Orientador: Prof. Dr. Rodrigo Bissacot \\ Coorientador: Prof. Dr. Aernout C.D. van Enter
}

Durante o desenvolvimento deste trabalho o autor recebeu auxílio financeiro de processos $n^{\circ} 2014 / 10637-9$ e n $2015 / 14434-8$,

Fundação de Amparo à Pesquisa do Estado de São Paulo (FAPESP)

São Paulo, setembro de 2018 


\section{Gibbs Measures for Models on Lines and Trees}

Esta versão da tese contém as correções e alterações sugeridas pela Comissão Julgadora durante a defesa da versão original do trabalho, realizada em 31/07/2018. Uma cópia da versão original está disponível no

Instituto de Matemática e Estatística da Universidade de São Paulo.

Comissão Julgadora:

- Prof. Dr. Rodrigo Bissacot - IME-USP

- Prof. Dr. Aernout C. D. van Enter - University of Groningen

- Prof. Dr. Luiz Renato G. Fontes - IME-USP

- Prof. Dr. Christof Kuelske - Ruhr-Universität Bochum

- Prof. Dr. Arnaud Le Ny - Université Paris-Est 


\section{Agradecimentos}

The best part of the thesis is here, since I can write about the people who I can thank a lot, the good moments of my Ph. D., and add anything that, in the future, when someone opens this part, he or she will see that I am grateful for these wonderful four years.

First I thank my supervisors, Aernout and Rodrigo, and my cosupervisor, Daniel, to open the door of statistical mechanics. Thanks to you, I found the area that I will hopefully follow for the rest of my life. I started studying this area when I was in the master degree, and Rodrigo gave a lecture on the introduction of models for ferromagnetism. He showed for the students that the statistical mechanics is not a rocket science, and it is actually a nice area. Thanks Rodrigo to give this amazing lecture. Aernout, who showed me that it is possible to think in the qualitative ideas avoiding heavy maths to understand how a problem could be solved, opened my mind. Thanks for many discussions that we had in Groningen. Daniel, I would like to thank you to have nice discussions about the spatial Gibbs random graphs problem, because those discussions were fruitful to understand how an idea and a proof can be simplified. Thanks to many advices and to help me how to "survive" in Groningen.

I would like to thank Noga Alon, Arnaud Le Ny and Wioletta Ruzsel to show me that to do research with collaborations is very interesting and delightful.

Let me thank Irene, who rented a room for me when I was in Groningen. The room is very confortable, and it was very important for me, since I could say "I'm home" in a country where I have never been before. Thanks to show me many dutch cultures, to help me to solve many bureaucracies, to teach dutch language, and for the dinners. Dank u wel, het was gezellig!

Why not also thanks Groningen city? Het is een mooie stad. Ik ben elke dag gefietst en ik altijd vond culturele evenementen. Ik vind Groningen leuk! Dank u wel, Groningen.

There are many people to thanks when I lived in the Netherlands. First, I would like to thank all of the group of the Groningen university. Desiree, Ineke and Lineke to help me for the university bureaucracy. Pedro, it was very fun to talk a lot with you, let's go again to eat krokketjes and drink dutch beer. Réka, it was so nice to show me nice places in Groningen and invite me to watch nice movies. Vladimír, thanks a lot to invite me to the game party and pancakes evenings. Further I would like to thanks Mónika, Nikolay, Pan and all other former and present members of our group. I would like to thanks Sylvia and Sonia for many nice talks that we had.

Bruno, you are brazilian, we met in Brazil, but it is so nice that we both went to the Netherlands at the same time to do our Ph. D. Thanks to show me Delft and the research group. Let's spread the brazilian's culture and memes in the world! Richard, thanks (again) to invite me to the workshop, let's have a drink again. I would like to thanks Dan, Federico, Willem and all former and present members of Delft group.

I met many people around the world when I participated in conferences, summer schools and 
workshops. I would like to thank all of them. Specially Albert, Carlo, Daniel Meissner, Henri, Imanishi, Lucas, Markus, Narçiçeği, Niimura, Raphael, Rene, Sangchul Lee, Sascha, Stjepan, Sungsoo Byun, Swee Hong Chan, Tóbiás, Tuan, Tulasi, and Zsolt. I thanks Kamijima, Kilian and Satoshi with whom we discussed a lot of statistical mechanics, lace expansion and some problems.

I would like to thank Marzio Cassandro, Brian Marcus, Akira Sakai, Siamak Taati, Philippe Thieullen and Utkir Rozikov for many helps and interesting discussions of Ising model's problems.

People from Brazil! There are a lot of people to thank here. First, Rodrigo's group. Elmer, Gregorio, João, Lucas, Luis, Luísa, Rodrigo Frausino and Thiago, the great group of statistical mechanics! Let me specially thanks Elmer, who we have many discussion about symbolic dynamics. Let's go again to a peruvian restaurant! Lucas, who show me interesting results of quantum statistical mechanics. I hope we will write a paper together!

Here, I would like to thank all of my brazilian friends. Rodrigo Cabral, who teaches me great results of operator algebra. When is it our next meeting in the bar? Caio, Diego, Feulo, Flipper, Lucas, Renatão and Vini, who we graduated the bachelor of mathematics together. Every semester we meet to have good drink in a nice restaurant, sometimes two or three times in a semester. When is our next meeting? Patrícia and Fabrício, thanks to many supports and talks.

I would like to thank Lídia and Oscar, who encourage me the authonomy of studies and selflearning.

I thank Fundação de Amparo à Pesquisa do Estado de São Paulo (FAPESP) to support me with the grants $n^{\circ} 2014 / 10637-9$ and $n^{\circ} 2015 / 14434-8$.

I would like to thank Kyomi, who supports me everyday. She walked with me together along my Ph. D. student's life, I shared my problems and happiness to her. Without her, I couldn't be here now.

I would like to finish the acknowledgments thanking my parents and all my relatives. Julio, Angélica, Lucy, Raphael and Seiya. I could continue doing my Ph. D. thanks to you. 


\section{Resumo}

ENDO, E. O. Medidas de Gibbs para modelos em retas e árvores. 2018. 98 f. Tese (Doutorado) - Instituto de Matemática e Estatística, Universidade de São Paulo, São Paulo, 2018.

Nesta tese estudamos diversas propriedades dos modelos de spins, em particular, os modelos de Ising e Dyson. Estudamos a estabilidade da transição de fase no modelo de Ising ferromagnético de primeiros vizinhos quando adicionamos uma perturbação no campo externo crítico pela qual se torna mais fraca ao estar distante da raiz da árvore de Cayley. Estudamos a relação entre $g$-medidas e medidas de Gibbs, mostrando que a medida de Gibbs do modelo de Dyson a temperaturas suficientemente baixas não é uma $g$-medida. Também estudamos contagem de contornos em árvores, mostramos uma caracterização das árvores que possuem um número infinito de contornos de um tamanho fixo envolvendo um vértice, e comparamos entre diversas definições de contornos. Estudamos também as medidas de grafos aleatórios spatial Gibbs, e suas convergências locais.

Palavras-chave: Modelo de Ising, modelo de Dyson, $g$-medidas, árvores de Cayley, medidas de Gibbs 



\section{Abstract}

ENDO, E. O. Gibbs Measures for Models on Lines and Trees. 2018. 98 f. Thesis (Ph. D.) Institute os Mathematics and Statistics, University of São Paulo, São Paulo, 2018.

In this thesis we study various properties of the spins models, in particular, Ising and Dyson models. We study the stability of the phase transition of the nearest-neighbor ferromagnetic Ising model when we add a perturbation to the critical external field that becomes weaker far from the root of the Cayley tree. We also study the relation between $g$-measures and Gibbs measures, showing that the Dyson model at sufficiently low temperature is not a $g$-measure. Counting contours on trees is also studied, showing the characterization of the trees that have infinite number of contours, and comparisons between various definitions of contours. We also study the measures of the spatial Gibbs random graphs, and their local convergence.

Keywords: Ising model, Dyson model, $g$-measures, Cayley trees, Gibbs measures 



\section{Contents}

$\begin{array}{ll}\text { List of Figures } & \text { i }\end{array}$

1 Introduction $\quad 1$

2 Spin Models

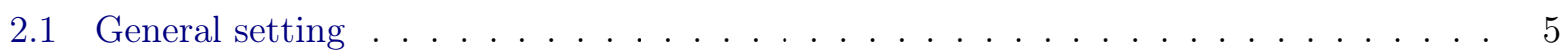

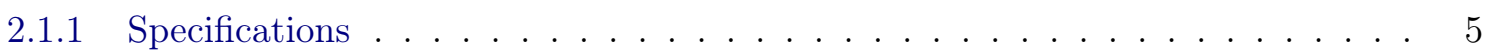

2.1 .2 Gibbsian specifications . . . . . . . . . . . . . . . . 7

$2.1 .3 \quad$ FKG inequality . . . . . . . . . . . . . . . . . . . . 9

2.2 Ising model . . . . . . . . . . . . . . . . . . . . . . . . . 11

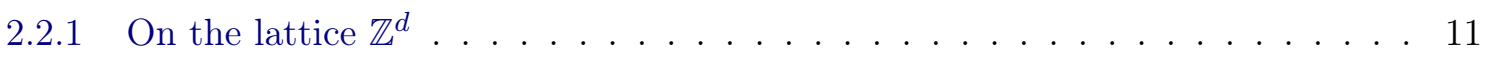

2.2 .2 On the Cayley Tree . . . . . . . . . . . . . . . . . . . . . 13

2.2 .3 Pressure and amenable graphs . . . . . . . . . . . . . . . 14

2.3 Dyson model . . . . . . . . . . . . . . . . . . . . . . . . . 15

3 Entropic repulsion and lack of the $g$-measure property for Dyson models $\quad 19$

3.1 Introduction . . . . . . . . . . . . . . . . . . . . . . . 19

3.2 Definitions and Notations . . . . . . . . . . . . . . . . . . . 20

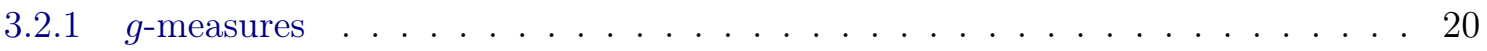

3.2.2 One-dimensional Gibbsian specification . . . . . . . . . . . . . . . 22

3.2.3 Not every $g$-measure is a Gibbs measure . . . . . . . . . . . . . . . . . 24

3.2.4 Gibbs vs $g$-measures for Dyson models in the Phase Transition region . . . . 24

3.2.5 Interfaces in Dyson models . . . . . . . . . . . . . . . . . 27

3.3 Entropic repulsion - Wetting transition . . . . . . . . . . . . . . . . . 30

3.4 Lack of the $g$-measure property: proof f . . . . . . . . . . . . . . . . . 34

3.5 Uncountable set of essentially discontinuous points . . . . . . . . . . . . . . 38

3.6 Final remarks and open questions . . . . . . . . . . . . . . . . . . . . 39

4 Stability of the Phase Transition of Critical-Field Ising Model on Cayley Trees 41

4.1 Introduction . . . . . . . . . . . . . . . . . . . . . 41

4.1 .1 Splitting Gibbs Measures . . . . . . . . . . . . . . . . . . . . . 42

4.2 Compatibility . . . . . . . . . . . . . . . . . . . . . 43

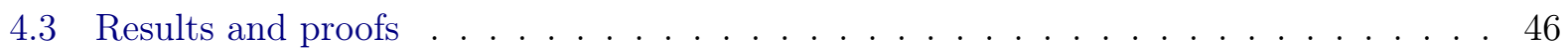

4.4 Final remarks and open questions . . . . . . . . . . . . . . . . . . . 52 
5 Counting Contours on Trees $\quad 53$

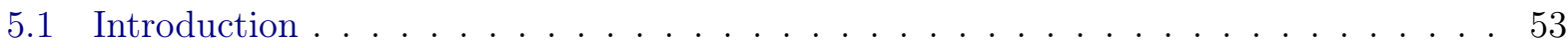

5.2 Definitions and Notations . . . . . . . . . . . . . . . . . 54

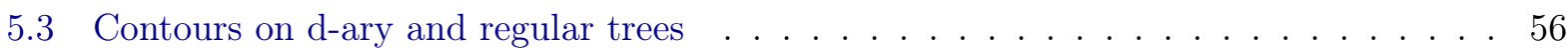

5.4 Infinitely many contours of size $n \ldots \ldots \ldots \ldots$. . . . . . . . . . . . . .

5.5 Appendix: Other definitions of contours and the phase transition . . . . . . . . 63

5.5 .1 Peierls contours . . . . . . . . . . . . . . . . . . 63

5.5 .2 Rozikov contours . . . . . . . . . . . . . . . . . . . . 67

5.6 Final remarks and open questions . . . . . . . . . . . . . . . . . . . . 69

6 Local Limits of Spatial Gibbs Random Graphs $\quad 71$

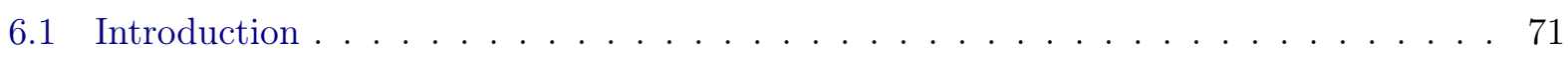

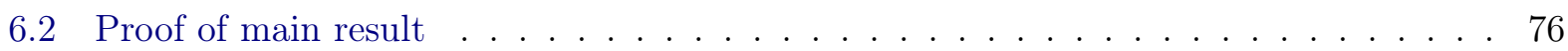

6.2.1 Truncated balls and proof of Theorem $42 \ldots \ldots \ldots \ldots$

6.2 .2 Estimates from $[132] \ldots \ldots \ldots \ldots$

6.2.3 Estimates for the reference measure and proof of Proposition 19 . . . . . . 82

6.3 No local convergence for $\gamma>1, p<\infty$ and $b>p+1 \ldots \ldots$. . . . . . . 85

6.4 Final remarks and open questions . . . . . . . . . . . . . 87

$\begin{array}{lr}\text { Bibliography } & 89\end{array}$ 


\section{List of Figures}

1.1 The spontaneous magnetisation, where $\beta_{c}$ is the inverse critical temperature. . . . . 2

3.1 Mesoscopic interval and the wet region. . . . . . . . . . . . . . 31

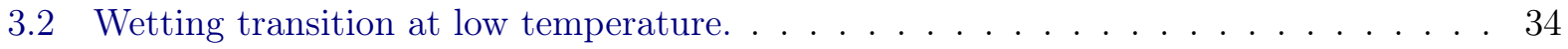

3.3 From wetting to essential discontinuity. Here $L_{1}=o(L)$ and $L N^{1-\alpha}=o(1) \ldots 37$

4.1 The graph of $\psi$ for $h=-h_{c}$ and the fixed points $b^{+}$and $b^{-}$, and the sequence $\psi^{n}(\infty)$

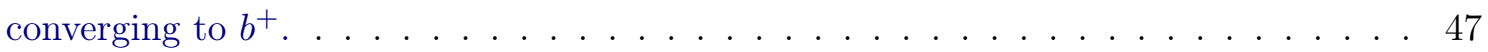

4.2 The Cayley tree with root $O$, and the auxiliary boundary fields. The circle means the depth of the tree. . . . . . . . . . . . . . . . . . . 50

5.1 Example of a contour of size four in a binary tree $T_{2} \ldots \ldots \ldots \ldots \ldots$

5.2 Example first iteration. . . . . . . . . . . . . . . . . . . . . . . 59

5.3 Part of the second iteration. . . . . . . . . . . . . . . . . . . . 59

5.4 Examples of connected component in $\mathcal{D}(\sigma) \ldots \ldots \ldots \ldots$. . . . . . . . . 64

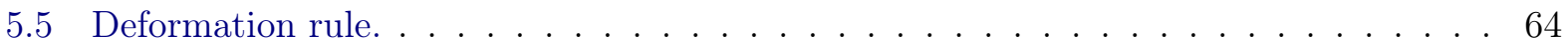

5.6 Set of contours on a finite set $\Lambda \ldots \ldots \ldots \ldots \ldots \ldots$

6.1 For each $p \in[1, \infty]$, the dark region represents the set $\mathcal{E}_{p}$, that is, the values of $b$ for which Theorem 41 does not cover the pair $(b, p)$ if $\gamma=1$. Note that, unless $p=1, \mathcal{E}_{p}$ only includes finitely many intervals and all numbers of the form $\frac{k-1}{k}, k \in \mathbb{N}$. . . . 72

6.2 Plot of the function $b \mapsto \alpha^{*}(\gamma, b)$ of Theorem 41 for the three cases $\gamma \in(0,1), \gamma>1$

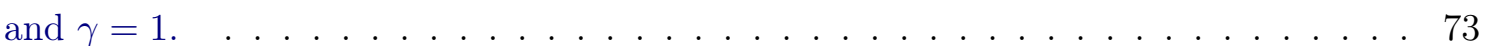

6.3 Hierarchical constructions that provide lower bounds for Theorem 41: case $\gamma<1$

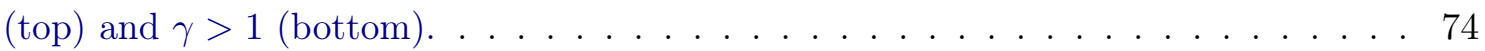

6.4 Hierarchical constructions that provide lower bounds for Theorem 41: case $\gamma=1$. . 74 



\section{Chapter 1}

\section{Introduction}

The Ising model, invented by Lenz and studied by Ising, is one of the first models that was studied in order to attempt to derive a phase transition by thermodynamical formalism. The model consists of configurations of molecules on a lattice $\mathbb{Z}^{d}$, in which each vertex $i$ is associated to a spin $\sigma_{i}$ which equals +1 or -1 (up or down). For each configuration $\sigma=\left(\sigma_{i}\right)_{i \in \mathbb{Z}^{d}} \in\{-1,1\}^{\mathbb{Z}^{d}}$, if two nearest-neighbor vertices on the lattice have the same spin values, then the interaction energy of the molecules is equal to $-J$, and if they are different, the interaction energy is equal to $J$. In other words, the interaction energy is equal to $-J \sigma_{i} \sigma_{j}$ for each nearest-neighbor pair $i$ and $j$ on $\mathbb{Z}^{d}$. In addition, for each spin we add the external magnetic field $-h$ if the spin is positive, and $h$ if it is negative. The formal Hamiltonian, which is the energy of a configuration, is given by

$$
H(\sigma)=-J \sum_{\langle i, j\rangle} \sigma_{i} \sigma_{j}-h \sum_{i \in \mathbb{Z}^{d}} \sigma_{i}
$$

where $\langle i, j\rangle$ means that $i$ and $j$ are nearest neighbors. If the coupling constant $J$ is positive, i.e., when the model is ferromagnetic, each spin tends to align with its neighbors and with the external magnetic field.

The partition function is given by

$$
Z_{\beta}=\sum_{\sigma \in\{-1,1\}^{Z^{d}}} e^{-\beta H(\sigma)}
$$

where $\beta=1 /(k T)$ is the inverse temperature, $k>0$ is the Boltzmann constant and $T>0$ is the temperature. The partition function is essential in statistical mechanics, since all thermodynamic functions come from it. In particular, the formal Gibbs measure is given by

$$
\mu_{\beta}(\sigma)=\frac{e^{-\beta H(\sigma)}}{Z_{\beta}}
$$

Note that the minus sign multiplying the Hamiltonian gives a high probability for the spins to have the same direction. In order to see the behavior of the spins, we measure the average of the spins, called magnetisation, projected along the direction of the magnetic field. Assume that a material is placed in a magnetic field. This means that each spin has a preference to point in the same direction. What happens when the magnetic field tends to zero? We have two different behaviors depending on the temperature. At high temperature, the material becomes paramagnetic, i.e., the magnetisation is zero, meaning that the spins are disordered; at low temperature, the material becomes ferromagnetic, meaning that the magnetisation remains positive (resp. negative) if the magnetic field approaches zero from the positive (resp. negative) side. Thus, the spins are ordered. This is called spontaneous magnetisation (this is especially when the field is zero, then there is a first-order transition in the magnetic field, that is a jump in the spontaneous magnetisation). Thus, there is a critical temperature, also known as the Curie temperature, separating the phases, 
corresponding to a phase transition.

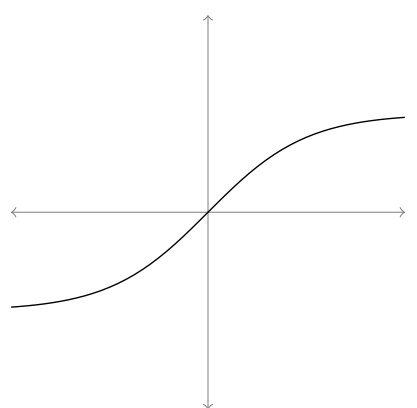

$\beta<\beta_{c}$.

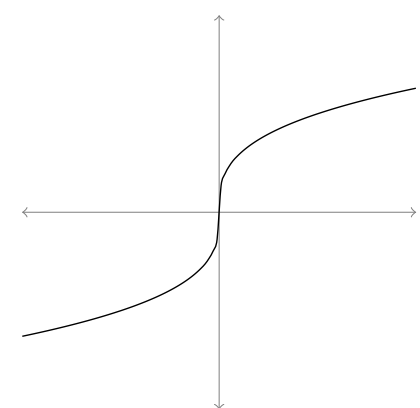

$\beta=\beta_{c}$.

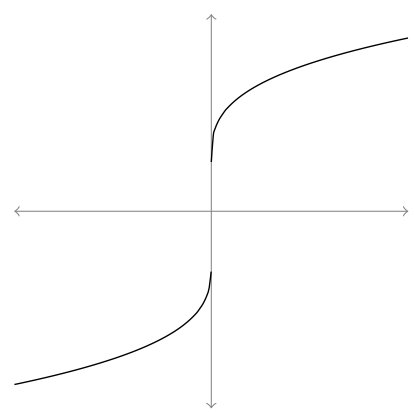

$\beta>\beta_{c}$.

Figure 1.1: The spontaneous magnetisation, where $\beta_{c}$ is the inverse critical temperature.

However, Ising [100], in 1922, showed that in the one-dimensional model the material is in a paramagnetic state at any temperature, since the ordered configuration is unstable, concluding that there is no phase transition. He did not realize that the argument holds only in one-dimensional case, and he also gave an idea of why the argument also should hold in two and three dimensions. Peierls published a paper showing that, contrary to Ising's prediciton, the model has a phase transition for the two- and three-dimensional lattices. He showed that, if we restrict the model in a plus boundary on a finite set, then, at low temperature, there are small regions of minus compared with the plus regions. The main technique to show it is the control of the number of the contours, invented by Peierls. Unfortunately, there was a incorrect step in the Peierls' proof, discovered by Fisher and Sherman. After being corrected, and proved for every dimension $d \geq 2$, this argument to show a phase transition is useful for many other models, and is now called the Peierls argument. For more details of the history of the Ising model, see [37].

Since the spins tend to be ordered when the magnetic field is non-null, it is natural to conjecture that the model has no phase transition when the absolute value of the magnetic field $|h|$ is big enough. Lee and Yang [125] showed more, they concluded that for every non-null $h$, the Ising model has no phase transition at any temperature. Also known as the Lee-Yang circle theorem, the proof uses complex analysis results, since they show that the pressure admits an analytic continuation to one of the regions

$$
\begin{aligned}
& H^{+}=\{z \in \mathbb{C}: \mathfrak{R e}(z)>0\}, \\
& H^{-}=\{z \in \mathbb{C}: \mathfrak{R e}(z)<0\} .
\end{aligned}
$$

The book of Ruelle [156] has an interesting chapter concerning this theorem.

Although the Ising model is recent, less than hundred years old, it has important contributions in the mathematical community, such as the work of Smirnov, who received the Fields medal in 2010 from the work in [160]. It was conjectured in 1990 that the scaling-limit of the bidimensional models in statistical mechanics is invariant under conformal mappings. Smirnov was the first to prove this rigorously in two different cases, percolation on the triangular lattice and the planar Ising model.

There is a variant of the Ising model called Dyson model, also known as Long-Range Ising model. Different from nearest-neighbour or short-range Ising models, the Dyson model has longrange interactions, this means that every two vertices $i$ and $j$ have a positive interaction of the form $J_{i j}=|i-j|^{-\alpha}$, where $\alpha \in(1,2]$. There are many results for this model $[3,4,39,41,54,55,62,77]$, such as the existence of a phase transition at low temperature, the notion of contours for the model, discontinuity at the critical temperature for $\alpha=2$ and continuity for $\alpha \in(1,2)$, phase-separation and the behavior of the interface point, and much more. In this thesis, we are going to present the relation between Gibbs measures and $g$-measures, which are measures which are compatible with a "one-sided specification" defined by Keane [112] in order to extend the theory of Markov chains of 
infinite order. The main result of [62] is that there exists a $g$-measure that is not a Gibbs measure. We are interested in studying the converse, proving that a low-temperature Gibbs measure for the Dyson model is not a $g$-measure.

Another work that we will present here, based on the paper [20], is the analysis of the speed of decay of an external magnetic field which depends on the vertices of a Cayley tree, thus an example of inhomogeneous external field, that converges to the critical external field $h_{c}>0$ identified by Preston [138], in order to see either a phase transition at low temperatures or the absence of the phase transition at any temperature in the nearest-neighbor Ising model. This problem is interesting due to the following reason. On the lattice $\mathbb{Z}^{d}$ with $d \geq 2$, as we explained before, Lee and Yang concluded the absence of a phase transition when there is a presence of an external magnetic field. Thus, instead of constant external field, if we consider the perturbed Ising model when each vertex $i$ is associated to an external field $h_{i}>0$ such that $h_{i}$ converges to zero when $i$ is going far from the origin, meaning that the model with the external field, far away from the origin, looks like the model without it, where there is a phase transition, how fast the external field should decay in order for the perturbed model still to undergo a phase transition? This problem is (partially) solved in $[20,45]$ when the external field is of the form $h_{i}=h^{*} \cdot\|i\|^{-\gamma}$, with $h^{*}>0$ and $\gamma>0$. We are going to present the work from [24], when, instead of the lattice $\mathbb{Z}^{d}$, we consider the Cayley tree.

Counting contours is an important combinatorial problem in statistical mechanics to have the possibility to show a phase transition in a model using a Peierls argument. Here we are going to count contours, defined by Babson and Benjamini [11], on trees. These contours, for graphs in general, are useful to estimate the critical probability for percolation on the graph. Although the critical probability for the tree is already known, the tools to estimate the number of contours are perhaps useful for find new ideas to control the number of contours of other graphs. We use the same idea as Balister and Bollobás [12] to compute the exact value of the number of contours on the regular trees and $d$-ary trees, and we compute an estimate for a family of trees where each vertex has at least $d$ children. We also characterize which trees have an infinite number of contours of a fixed size. In fact, since a Peierls argument needs that the number of contours of a finite size should be finite, we cannot apply the argument for those type of trees.

The presence of hierarchical structures in the connectivity network of neurons in the brain or of the Internet's routers [7, 113, 162] raises the question to define a model that captures it. Mourrat and Valesin [132] defined Gibbs-type measures on the lattice $\mathbb{Z}$ capturing the fundamental interplay between the geometry of the graph and of the underlying space. In a few words, the measure is over the long edges on $\mathbb{Z}$, where a typical graph on a finite interval avoids long edges and also large diameter. Here, we are going to show the local convergence properties of this measure.

In this thesis we present the works $[9,23,24,56]$. We organize the thesis in the following way.

1. Chapter 2: We present the Gibbsian specifications, FKG inequality, Ising model on the lattice $\mathbb{Z}^{d}$ and on the Cayley tree, and Dyson model. We discuss some classical and recent results.

2. Chapter 3: We present the work [23], showing that the Gibbs measures of the Dyson model at sufficiently low temperature are not $g$-measures.

3. Chapter 4: We present the work [24]. We add a spatially dependent inhomogeneous external field to the ferromagnetic Ising model on a Cayley tree, and we show the phase diagram depending on the decay of the external field.

4. Chapter 5: We present the work [9]. We count and estimate the number of contours on a family of trees, show a characterization of trees that has infinite number of contours of a fixed size, and we compare with the other definitions of contours, such as Peierls contours and Rozikov contours.

5. Chapter 6: We present the work [56]. We show the local limit behaviour of the spatial Gibbs random graphs defined in [132]. 



\section{Chapter 2}

\section{Spin Models}

\subsection{General setting}

\subsubsection{Specifications}

Let $S$ be a compact metric space called the state space, and let $\mathcal{S}$ be the $\sigma$-algebra of the state space $S$. For instance, when $S=\{-1,1\}$, we choose $\mathcal{S}=\mathcal{P}(S)$ to be the power set of $S$. Let $G$ be an infinite, locally finite and connected graph, for instance, the lattice $G=\mathbb{Z}^{d}$ or the Cayley tree $G=\Gamma^{d}$, which is the $d+1$-regular tree. Consider also $(\Omega, \mathcal{F})$ to be a measurable space, where $\Omega=S^{G}$ with $d \geq 1$ and let $\mathcal{F}$ be the $\sigma$-algebra generated by cylinder sets. Let us denote the set of probability measures on $(\Omega, \mathcal{F})$ by $\mathcal{M}_{1}(\Omega, \mathcal{F})$. For a fixed set $\Lambda \subset G, i \in \Lambda$ and $F \in \mathcal{S}$, we define

$$
C_{F}^{i}=\left\{\sigma \in \Omega: \sigma_{i} \in F\right\}
$$

Moreover, consider the set

$$
\mathcal{C}_{\Lambda}=\left\{C_{F}^{i}: i \in \Lambda, F \in \mathcal{S}\right\}
$$

We define $\mathcal{F}_{\Lambda}$ be the smallest sub- $\sigma$-algebra of $\mathcal{F}$ generated by $\mathcal{C}_{\Lambda}$.

For a finite set $\Lambda \subset G$, which we denote by $\Lambda \Subset G$, the set $C\left(\Omega, \mathcal{F}_{\Lambda}\right)$ is the set of $\mathcal{F}_{\Lambda}$-measurable continuous functions $f: \Omega \rightarrow \mathbb{R}$. We also denote by $\mathscr{L}$ be the set of all finite subsets of $G$.

A potential is a family $\Phi=\left\{\Phi_{A}\right\}_{A \in \mathscr{L}}$ of functions indexed by $\mathscr{L}$, where $\Phi_{A} \in C\left(\Omega, \mathcal{F}_{A}\right)$ for all $A \in \mathscr{L}$.

We denote by $\left\|\Phi_{A}\right\|_{\infty}$ the supremum norm of $\Phi_{A}$, i.e.,

$$
\left\|\Phi_{A}\right\|_{\infty}=\sup _{\sigma \in \Omega}\left|\Phi_{A}(\sigma)\right| .
$$

We say that the potential is absolutely summable if

$$
\sum_{\substack{A \ni i \\ A \in \mathscr{L}}}\left\|\Phi_{A}\right\|_{\infty}<\infty \quad \text { for every } i \in G .
$$

We define the Hamiltonian for every finite set $\Lambda \Subset G$ by

$$
H_{\Lambda}(\sigma)=\sum_{\substack{A \Subset G \\ A \cap \Lambda \neq \emptyset}} \Phi_{A}(\sigma) .
$$

Let us give an example of an absolutely summable potential. Let us consider $\Omega=\{-1,1\}^{G}$ and the 
following potential $\Phi=\left\{\Phi_{A}\right\}_{A \in \mathscr{L}}$,

$$
\Phi_{A}(\sigma)= \begin{cases}-J_{i j} \sigma_{i} \sigma_{j}, & \text { if } A=\{i, j\}, \\ -h_{i} \sigma_{i}, & \text { if } A=\{i\}, \\ 0, & \text { otherwise }\end{cases}
$$

where $J_{i j}$ and $h_{i}$ are real-valued numbers. The family $\left(J_{i j}\right)_{i, j \in G}$ is called set of coupling constants and $\left(h_{i}\right)_{i \in G}$ is called external field. The model associated to the potential $\Phi$ given in (2.6) is called an Ising model with pair interactions $\left(J_{i j}\right)_{i, j \in G}$ and external field $\left(h_{i}\right)_{i \in G}$. We say that the Ising model is ferromagnetic if $J_{i j} \geq 0$ for every $i, j \in G$.

For each $i, j \in G$, define the distance $\mathrm{d}(i, j)$ to be the smallest length of the paths (self-avoiding walks) from $i$ to $j$. Since $G$ is a connected graph, the distance is well-defined. We say that the Ising model is:

1. Nearest-neighbor if $J_{i j}=0$ for every d $(i, j)>1$.

2. Short-range if there exists $R>0$ such that $J_{i j}=0$ for every $\mathrm{d}(i, j)>R$.

3. Long-range if the model is not short-range.

It is easy to show that this potential is absolutely summable if, and only if,

$$
\left|h_{i}\right|+\sum_{\substack{j \in G \\ j \neq i}}\left|J_{i j}\right|<\infty
$$

for every $i \in G$.

For two configurations $\sigma, \omega \in \Omega$ and a finite set $\Lambda \Subset G$, let us define the configuration $\sigma_{\Lambda} \in S^{\Lambda}$ by $\sigma_{\Lambda}=\left(\sigma_{i}\right)_{i \in \Lambda}$, and the concatenation $\sigma_{\Lambda} \omega_{\Lambda^{c}}$ by

$$
\left(\sigma_{\Lambda} \omega_{\Lambda^{c}}\right)_{i}= \begin{cases}\sigma_{i}, & \text { if } i \in \Lambda, \\ \omega_{i}, & \text { if } i \in \Lambda^{c}\end{cases}
$$

where $\Lambda^{c}=G \backslash \Lambda$ is the complement of $\Lambda$.

Definition 1. Let $(\Omega, \mathcal{F})$ be a measurable space and $\Lambda \Subset G$. A probability kernel is a map $\gamma_{\Lambda}$ : $\mathcal{F} \times \Omega \rightarrow[0,1]$ with the following properties:

1. For every $\omega \in \Omega, \gamma_{\Lambda}(\cdot \mid \omega)$ is a probability measure on $(\Omega, \mathcal{F})$.

2. For every $F \in \mathcal{F}, \gamma_{\Lambda}(F \mid \cdot)$ is $\mathcal{F}_{\Lambda^{c}}$ measurable.

If, moreover,

$$
\gamma_{\Lambda}(F \mid \omega)=\mathbb{1}_{F}(\omega) \text { for every } \mathcal{F}_{\Lambda^{c}-\text { measurable set } F,}
$$

for all $\omega \in \Omega$, then $\gamma_{\Lambda}$ is called proper.

Consider a finite set $\Lambda \Subset G$ and $\omega \in \Omega$. Consider the set

$$
\Omega_{\Lambda}^{\omega}=\left\{\sigma \in \Omega: \sigma_{i}=\omega_{i} \text { for every } i \notin \Lambda\right\} .
$$

Note that

$$
\gamma_{\Gamma}\left(\Omega_{\Lambda}^{\omega} \mid \omega\right)=\mathbb{1}_{\Omega_{\Lambda}^{\omega}}(\omega)=1
$$

We will call $\omega$ the boundary condition of $\gamma_{\Lambda}(\cdot \mid \omega)$.

Definition 2. A specification is a family $\left\{\gamma_{\Lambda}\right\}_{\Lambda \in \mathscr{L}}$ of proper probability kernels that is consistent, i.e., for every $\Lambda \subseteq \Gamma \Subset G$, and $f: \Omega \rightarrow \mathbb{R}$ be a $\mathcal{F}$-measurable bounded function, and $\eta \in \Omega$, we have

$$
\int_{\Omega} \int_{\Omega} f\left(\sigma_{\Lambda} \omega_{\Gamma \backslash \Lambda} \eta_{\Gamma^{c}}\right) \gamma_{\Lambda}\left(\mathrm{d} \sigma \mid \omega_{\Gamma} \eta_{\Gamma^{c}}\right) \gamma_{\Gamma}(\mathrm{d} \omega \mid \eta)=\int_{\Omega} f\left(\omega_{\Gamma} \eta_{\Gamma^{c}}\right) \gamma_{\Gamma}(\mathrm{d} \omega \mid \eta) .
$$


The equation (2.12) is called a condition of compatibility, and we will also refer to this equation by the following notation,

$$
\gamma_{\Lambda} \gamma_{\Gamma}=\gamma_{\Gamma}
$$

for every $\Lambda \subseteq \Gamma \Subset G$. See e.g. [61, 65, 84, 86, 139, 161] for more details about specifications.

\subsubsection{Gibbsian specifications}

The main example of a specification is the Gibbsian specification shown below. We first take a probability measure $\nu$ on $(S, \mathcal{S})$ called a priori measure.

Theorem 1. Let $(S, \mathcal{S}, \nu)$ be a probability space and $\Phi$ be an absolutely summable potential defined on $(\Omega, \mathcal{F})$. For a fixed inverse temperature $\beta>0$, a finite set $\Lambda \Subset G$, and $F \in \mathcal{F}$, the expressions

$$
\mu_{\Lambda, \beta}^{\omega}(F)=\frac{1}{Z_{\Lambda, \beta}^{\omega}} \int_{S^{\Lambda}} \mathbb{1}_{F}\left(\sigma_{\Lambda} \omega_{\Lambda^{c}}\right) \cdot e^{-\beta H_{\Lambda}\left(\sigma_{\Lambda} \omega_{\Lambda^{c}}\right)} \prod_{i \in \Lambda} \mathrm{d} \nu\left(\sigma_{i}\right)
$$

where

$$
Z_{\Lambda, \beta}^{\omega}=\int_{S^{\Lambda}} e^{-\beta H_{\Lambda}\left(\sigma_{\Lambda} \omega_{\Lambda^{c}}\right)} \prod_{i \in \Lambda} \mathrm{d} \nu\left(\sigma_{i}\right),
$$

define a local specification, called Gibbsian specification for potential $\Phi$ with inverse temperature $\beta$.

The reader can find the proof of Theorem 1 in [28, 75, 84]. Let us also define the free boundary condition. For a fixed $\Lambda \Subset G$, define the Hamiltonian of the free boundary condition by

$$
H_{\Lambda}^{\text {free }}\left(\sigma_{\Lambda}\right)=\sum_{A \subset \Lambda} \Phi_{A}\left(\sigma_{\Lambda}\right)
$$

Let $\left(\Omega_{\Lambda}, \mathcal{F}_{\Lambda}\right)$ be a measurable space, where

$$
\Omega_{\Lambda}=\left\{\sigma_{\Lambda}=\left(\sigma_{i}\right)_{i \in \Lambda}: \sigma_{i} \in\{-1,1\}\right\} .
$$

If $F$ is $\mathcal{F}_{\Lambda}$-measurable, the measure

$$
\mu_{\Lambda, \beta}^{\text {free }}(F)=\frac{1}{Z_{\Lambda, \beta}^{\text {free }}} \int_{S^{\Lambda}} \mathbb{1}_{F}\left(\sigma_{\Lambda}\right) \cdot e^{-\beta H_{\Lambda}^{\text {free }}\left(\sigma_{\Lambda}\right)} \prod_{i \in \Lambda} \mathrm{d} \nu\left(\sigma_{i}\right),
$$

where

$$
Z_{\Lambda, \beta}^{\mathrm{free}}=\int_{S^{\Lambda}} e^{-\beta H_{\Lambda}^{\mathrm{free}}\left(\sigma_{\Lambda}\right)} \prod_{i \in \Lambda} \mathrm{d} \nu\left(\sigma_{i}\right)
$$

also defines a local specification. We call the measure $\mu_{\Lambda, \beta}^{\omega}$ the finite-volume Gibbs measure with boundary condition $\omega$, volume $\Lambda$ and inverse temperature $\beta$.

When $S=\{-1,1\}$ and $\mathcal{S}=\mathcal{P}(S)$ is the power set of $S$, the a priori measure is the product measure of the single-site measure $\nu=\frac{1}{2} \delta_{+}+\frac{1}{2} \delta_{-}$, where, for every $i \in G$, the function $\delta_{+}$(resp. $\left.\delta_{-}\right)$is the indicator function

$$
\delta_{ \pm}\left(\sigma_{i}\right)= \begin{cases}1, & \text { if } \sigma_{i}= \pm 1 \\ 0, & \text { otherwise }\end{cases}
$$

and $\Phi$ the potentials defined in (2.6), the probability measure $\mu_{\Lambda, \beta}^{\omega}$ is the finite-volume Gibbs measure of the Ising model.

The expectation value of a function $f: \Omega_{\Lambda}^{\omega} \rightarrow \mathbb{R}$ under $\mu_{\Lambda, \beta}^{\omega}$ is denoted by

$$
\mu_{\Lambda, \beta}^{\omega}(f)=\sum_{\sigma \in \Omega_{\Lambda}^{\omega}} f(\sigma) \mu_{\Lambda, \beta}^{\omega}(\sigma)
$$

We will sometimes denote the expectation value as $\langle f\rangle_{\Lambda, \beta}^{\omega}$. 
A function $f: \Omega \rightarrow \mathbb{R}$ is continuous at $\sigma \in \Omega$ if, for every $\varepsilon>0$, there exists $\Lambda \Subset G$ such that

$$
\sup _{\omega \in \Omega}\left|f\left(\sigma_{\Lambda} \omega_{\Lambda^{c}}\right)-f(\sigma)\right|<\varepsilon
$$

Moreover, $f$ is continuous if $f$ is continuous at every $\sigma \in \Omega$.

A function $f: \Omega \rightarrow \mathbb{R}$ is local if there exists a finite set $\Delta \subset G$ such that, for every $\sigma, \sigma^{\prime} \in \Omega$ with $\sigma_{i}=\sigma_{i}^{\prime}$ for $i \in \Delta$, we have $f(\sigma)=f\left(\sigma^{\prime}\right)$. The smallest such set $\Delta$ is called the support of $f$ and it is denoted by $\operatorname{supp}(f)$.

A function $f: \Omega \rightarrow \mathbb{R}$ is quasilocal if there exists a sequence of local functions $\left(f_{n}\right)_{n \geq 1}$ such that $\left\|f_{n}-f\right\|_{\infty} \rightarrow 0$ as $n \rightarrow \infty$. When the state space $S$ is compact and discrete, quasilocal functions and continuous functions are equivalent, see [75] (More generally, for compact state space $S$, continuous functions are quasilocal, and for discrete state space $S$, quasilocal functions are continuous). A specification $\left\{\gamma_{\Lambda}\right\}_{\Lambda \in \mathscr{L}}$ is called quasilocal if each probability kernel $\gamma_{\Lambda}$ is continuous with respect to its boundary condition. In other words, for all $F \in \mathcal{F}$, the map $\omega \mapsto \gamma_{\Lambda}(F \mid \omega)$ is continuous. If $\Phi$ is an absolutely summable potential, then the Gibbsian specification $\left\{\mu_{\Lambda, \beta}^{(\cdot)}\right\}_{\Lambda \in \mathscr{L}}$ for potential $\Phi$ with inverse temperature $\beta$ on $(\Omega, \mathcal{F})$ is quasilocal. See e.g. [58, 84, 118, 166]. In fact, in the context of possibly non-Gibbsian renormalized Gibbs measures [58, 59], the major characterisation used of the latter was precisely the lack of this quasilocality property (as well as the main drawback, preventing many standard results).

Let us now define the Gibbs measure given a potential $\Phi$ by using a Gibbsian specification. For this, for a fixed probability measure $\mu$ on a probability space $(\Omega, \mathcal{F})$ and $\mathcal{B}$ be a sub- $\sigma$-algebra of $\mathcal{F}$, we will write $\mu(\cdot \mid \mathcal{B}):=\mathbb{E}_{\mu}(\cdot \mid \mathcal{B})$ for the conditional probability.

Definition 3. Let $(S, \mathcal{S}, \nu)$ be a probability space and $\Phi$ be an absolutely summable potential defined on $(\Omega, \mathcal{F})$. For a fixed inverse temperature $\beta>0$, a probability measure $\mu_{\beta}$ is called Gibbs measure associated to the potential $\Phi$ if, for every $\Lambda \Subset G$ and $F \in \mathcal{F}$, we have

$$
\mu_{\beta}\left(F \mid \mathcal{F}_{\Lambda^{c}}\right)(\omega)=\mu_{\Lambda, \beta}^{\omega}(F) \quad \mu_{\beta}-\text { a.e. }
$$

The set of all Gibbs measures satisfying the above conditions is denoted by $\mathcal{G}_{\beta}^{\mathrm{DLR}}(\Phi)$. Note that $\mathcal{G}_{\beta}^{\mathrm{DLR}}(\Phi)$ is closed and convex (See $[28,84,75]$ ). Every Gibbs measure associated to some absolutely summable potential $\Phi$ satisfies the so called DLR-equation (namely for all volumes and almost all boundary conditions).

Theorem 2. Let $\left\{\mu_{\Lambda, \beta}^{(\cdot)}\right\}_{\Lambda \in \mathscr{L}}$ be a Gibbsian specification for potential $\Phi$ with inverse temperature $\beta$ on $(\Omega, \mathcal{F})$. A probability measure $\mu_{\beta} \in \mathcal{M}_{1}(\Omega, \mathcal{F})$ is a Gibbs measure associated to the regular potential $\Phi$ if, and only if,

$$
\mu_{\beta} \mu_{\Lambda, \beta}^{(\cdot)}=\mu_{\beta}
$$

for every finite set $\Lambda \Subset G$, i.e., for every local function $f$,

$$
\int_{\Omega} \int_{\Omega} f(\sigma) \mathrm{d} \mu_{\Lambda, \beta}^{\omega}(\mathrm{d} \sigma) \mu_{\beta}(\mathrm{d} \omega)=\int_{\Omega} f(\sigma) \mu_{\beta}(\mathrm{d} \sigma)
$$

The equations (2.24) are called DLR equations, in tribute to Dobrushin, Lanford and Ruelle. The so-called DLR approach is described also for example in [58, 75, 84, 101, 139]. The next theorem guarantees that every thermodynamical limit of a finite-volume Gibbs measures is a Gibbs measure. Given a sequence of finite sets $\left(\Lambda_{n}\right)_{n \geq 1}$, we write $\Lambda_{n} \uparrow G$ if for every $i \in \mathbb{Z}^{d}$ there exists $n_{0} \geq 1$ such that, for every $n \geq n_{0}$, we have $i \in \Lambda_{n}$. For given a sequence $\Lambda_{n} \uparrow G$ and $\left(\omega_{n}\right)_{n \geq 1}$, we say that a sequence of measures $\mu_{\Lambda_{n}, \beta}^{\omega_{n}}$ converges weakly to a probability measure $\mu$ if for every local function $f$, we have

$$
\lim _{n \rightarrow \infty} \mu_{\Lambda_{n}, \beta}^{\omega_{n}}(f)=\mu(f) .
$$

We will write $\lim _{n \rightarrow \infty} \mu_{\Lambda_{n}, \beta}^{\omega_{n}}=\mu$ for the weak limit. 
Theorem 3. Let $\Phi$ be a potential and $\beta$ be the inverse temperature. Let $\left(\Lambda_{n}\right)_{n \geq 1}$ be a sequence of finite sets on $G$ such that $\Lambda_{n} \uparrow G$. If, for some sequence $\omega_{n} \in \Omega$, the sequence of finite-volume Gibbs measures $\mu_{\Lambda_{n}, \beta}^{\omega_{n}}$ converges weakly to some probability measure $\mu$, then $\mu$ is a Gibbs measure associated to the absolutely summable potential $\Phi$.

Define $\mathcal{G}_{\beta}(\Phi)$ to be the closed convex hull of the set

$$
\left\{\mu \in \mathcal{M}_{1}(\Omega, \mathcal{F}): \text { there exists } \Lambda_{n} \uparrow G \text { and }\left(\omega_{n}\right)_{n \geq 1} \text { such that } \lim _{n \rightarrow \infty} \mu_{\Lambda_{n}, \beta}^{\omega_{n}}=\mu\right\} .
$$

Due to the compactness of the state space, the set $\mathcal{G}_{\beta}(\Phi)$ is non-empty since there always exist convergent subsequences. Clearly $\mathcal{G}_{\beta}(\Phi) \subseteq \mathcal{G}_{\beta}^{\mathrm{DLR}}(\Phi)$. Moreover, the sets are equal.

Theorem 4. Let $\left\{\mu_{\Lambda, \beta}^{(\cdot)}\right\}_{\Lambda \in \mathscr{L}}$ be a Gibbsian specification for potential $\Phi$ with inverse temperature $\beta$ on $(\Omega, \mathcal{F})$. Then

$$
\mathcal{G}_{\beta}(\Phi)=\mathcal{G}_{\beta}^{\mathrm{DLR}}(\Phi)
$$

We added the convex-hull in the definition of $\mathcal{G}_{\beta}(\Phi)$, but it is a natural question if the set $\mathcal{G}_{\beta}(\Phi)$ is already a convex set. Coquille [47] showed that, in the ferromagnetic nearest-neighbor Ising model on the lattice $\mathbb{Z}^{3}$, there exists a Gibbs measure which is not a thermodynamical limit of any sequence of Gibbs measures with boundary conditions.

The model has non-uniqueness at $\beta$, or undergoes a phase transition at $\beta$ if $\left|\mathcal{G}_{\beta}(\Phi)\right|>1$, and the model has uniqueness at $\beta$, or absence of phase transition at $\beta$, if $\left|\mathcal{G}_{\beta}(\Phi)\right|=1$.

Since the potential $\Phi$ is absolutely summable, by Dobrushin's Uniqueness Theorem [50], there exists $\beta_{c}>0$ such that the model has uniqueness for every $\beta<\beta_{c}$. The phenomenon of the phase transition depends on the model that we consider. In the next section we will present the Ising model and Dyson model, which undergoes a phase transition at low temperatures. However, not every model has a phase transition, for instance, the one-dimensional ferromagnetic nearest-neighbor Ising model is an example of the absence of phase transition at any temperature.

A Gibbs measure $\mu \in \mathcal{G}_{\beta}(\Phi)$ is extremal if $\mu$ is not a nontrivial convex combination of any other Gibbs measures, i.e., for every $\mu_{1}, \mu_{2} \in \mathcal{G}_{\beta}(\Phi)$ such that $\mu=\alpha \mu_{1}+(1-\alpha) \mu_{2}$ for some $\alpha \in[0,1]$, then $\alpha \in\{0,1\}$. Let $\operatorname{ex}\left(\mathcal{G}_{\beta}(\Phi)\right)$ be the set of all extremal Gibbs measures. By Krein-Millman Theorem [119], we have

$$
\mathcal{G}_{\beta}(\Phi)=\overline{\operatorname{conv}}\left(\operatorname{ex}\left(\mathcal{G}_{\beta}(\Phi)\right)\right)
$$

i.e., the set of all Gibbs measures is the closed convex hull of the set of all extremal Gibbs measures.

We use the term Gibbs measure in the Statistical Mechanics sense, as defined by Dobrushin, Lanford and Ruelle [50, 122]. In the Dynamical Systems community, often a somewhat different notion of Gibbs measure is defined following Sinai, Ruelle and Bowen [30, 155, 159], by providing uniformly bounded approximations of the measure on cylinders as exponential Boltzmann-Gibbs weights defined via (a slightly different notion of) potentials.

In symbolic dynamics, yet another notion is introduced either via Perron-Frobenius operators or via variational principles and a corresponding notion of equilibrium states. Compare e.g. [18] with sometimes different (non-)lattices, and again different notions of potentials compared to the ones used in Mathematical Statistical Mechanics. This yields different, typically more restrictive, classes of measures, in which phase transitions are usually excluded due to the corresponding potential being too short-range (in statistical mechanics terms). For example, a potential with summable variations defined on $\{-1,+1\}^{\mathbb{N}}$ admits a unique equilibrium measure, see [30, 153].

\subsubsection{FKG inequality}

Correlation inequalities are a fundamental tool to analyse various statistical mechanics models. There are several correlations inequalities in the literature, but one of them, called FKG inequality, after Fortuin, Kasteleyn and Ginibre [72], plays a particularly important role to show essential results in the area. We will present the version by Preston [140], who included the case of continuous spins. 
Let $\Lambda$ be a finite set, and for each $i \in \Lambda$, let $\left(X_{i}, \mathcal{F}_{i}, \nu_{i}\right)$ be a measure space with $\nu_{i}$ a nonnegative $\sigma$-finite measure. Suppose that $X_{i}$ is equipped with a total order $\geq$ that is $\mathcal{F}_{i}$-measurable, i.e.,

$$
\left\{\left(x_{i}, y_{i}\right) \in X_{i} \times X_{i}: x_{i} \geq y_{i}\right\} \in \mathcal{F}_{i} \times \mathcal{F}_{i} .
$$

Let us denote $X=\prod_{i \in \Lambda} X_{i}$ and the corresponding $\sigma$-algebra $\mathcal{F}=\prod_{i \in \Lambda} \mathcal{F}_{i}$, and let $\nu=\prod_{i \in \Lambda} \nu_{i}$. Suppose $f_{1}, f_{2}: X \rightarrow \mathbb{R}$ are $\mathcal{F}$-measurable with the properties

(1) $f_{1}, f_{2} \geq 0$

(2) $\int_{X} f_{1} \mathrm{~d} \nu=\int_{X} f_{2} \mathrm{~d} \nu=1$.

For $t=1,2$ let $\mu_{t}$ denote the probability measure $f_{t} \mathrm{~d} \nu$ on $(X, \mathcal{F})$. If $x=\left(x_{i}\right)_{i \in \Lambda}$ and $y=\left(y_{i}\right)_{i \in \Lambda}$, we define

$$
\begin{aligned}
& x \wedge y=\left(\min \left(x_{i}, y_{i}\right)\right)_{i \in \Lambda}, \\
& x \vee y=\left(\max \left(x_{i}, y_{i}\right)\right)_{i \in \Lambda} .
\end{aligned}
$$

We write $x \leq y$ if $x_{i} \leq y_{i}$ for every $i \in \Lambda$. We say that a function $f: X \rightarrow \mathbb{R}$ is nondecreasing if, for every $x \leq y$, we have $f(x) \leq f(y)$.

Theorem 5. Suppose $f_{1}, f_{2}$ satisfy

$$
f_{1}(x \wedge y) f_{2}(x \vee y) \geq f_{1}(x) f_{2}(y) \quad \text { for all } \quad x, y \in X .
$$

If $h: X \rightarrow \mathbb{R}$ is bounded, $\mathcal{F}$-measurable and nondecreasing, then

$$
\int_{X} h \mathrm{~d} \mu_{1} \geq \int_{X} h \mathrm{~d} \mu_{2}
$$

The Gibbs measures of the ferromagnetic Ising model (defined in (2.6)) satisfy the FKG inequality. Now, we will write the finite-volume Gibbs measures of the ferromagnetic Ising model as $\mu_{\Lambda, \beta, \bar{h}}^{(\cdot)}$.

Theorem 6. Let $\Lambda \Subset \mathbb{Z}^{d}$ be a finite set, $\beta>0,\left(h_{i}\right)_{i \in \mathbb{Z}^{d}},\left(J_{i j}\right)_{i, j \in G}$ with $J_{i j} \geq 0$ for every $i, j \in G$ and $\omega \in \Omega$ be any boundary condition. Then, for every pair of nondecreasing functions $f$ and $g$,

$$
\mu_{\Lambda, \beta, \bar{h}}^{\omega}(f g) \geq \mu_{\Lambda, \beta, \bar{h}}^{\omega}(f) \mu_{\Lambda, \beta, \bar{h}}^{\omega}(g) .
$$

We call plus boundary condition when $\omega_{i}=+1$ for every $i \in G$ and minus boundary condition when $\omega_{i}=-1$ for every $i \in G$, and we will denote the Gibbs measures, respectively, by $\mu_{\Lambda, \beta, \bar{h}}^{+}$ and $\mu_{\Lambda, \beta, \bar{h}}^{-}$.

By Theorem 6, there exist thermodynamical limits for plus, minus and free boundary conditions,

$$
\mu_{\beta, \bar{h}}^{+}:=\lim _{n \rightarrow \infty} \mu_{\Lambda_{n}, \beta, \bar{h}}^{+}, \quad \mu_{\beta, \bar{h}}^{-}:=\lim _{n \rightarrow \infty} \mu_{\Lambda_{n}, \beta, \bar{h}}^{-} \quad \text { and } \quad \mu_{\beta, \bar{h}}^{\text {free }}:=\lim _{n \rightarrow \infty} \mu_{\Lambda_{n}, \beta, \bar{h}}^{\text {free }},
$$

for every sequence $\Lambda_{n} \uparrow G$. Moreover, by Theorem 6 , we can show that $\mu_{\Lambda, \beta, \bar{h}}^{-}$is stochastically dominated by $\mu_{\Lambda, \beta, \bar{h}}^{\omega}$, and $\mu_{\Lambda, \beta, \bar{h}}^{\omega}$ is stochastically dominated by $\mu_{\Lambda, \beta, \bar{h}}^{+}$, for every $\omega \in\{-1,1\}^{G}$. See [75] for the proof.

Corollary 1. Let $f$ be an arbitrary nondecreasing function. Then, for any $\beta>0$,

$$
\mu_{\Lambda, \beta, \bar{h}}^{-}(f) \leq \mu_{\Lambda, \beta, \bar{h}}^{\omega}(f) \leq \mu_{\Lambda, \beta, \bar{h}}^{+}(f),
$$

for any boundary condition $\omega$ (including the free boundary condition) and any $\Lambda \Subset G$.

Let us emphasize the external field in the set of Gibbs measures $\mathcal{G}_{\beta}(\Phi)=\mathcal{G}_{\beta, \bar{h}}$. Using Corollary 1 , we have the following equivalence. 
Theorem 7. Let $\beta>0$ and $\bar{h}=\left(h_{i}\right)_{i \in G}$. The following statements are equivalent:

1. There is absence of phase transition at $(\beta, \bar{h})$.

2. $\mu_{\beta, \bar{h}}^{+}=\mu_{\beta, \bar{h}}^{-}$.

There are several generalizations or adaptations of the FKG inequality in the literature, see [1, $68,95,116,143,144,157,170]$. The other correlation inequality that is similar to the FKG inequality is the Griffiths inequality, also known as GKS inequality, after Griffiths, Kelly and Sherman [89, 90, 114].

\section{$2.2 \quad$ Ising model}

We are going to present some classical results of the Ising model, defined by the potential (2.6), on the lattice $\mathbb{Z}^{d}$ and on the Cayley tree $\Gamma^{d}$. The Hamiltonian of the ferromagnetic Ising model in a finite set $\Lambda \Subset G$ with boundary condition $\omega$ is a function on $\Omega_{\Lambda}^{\omega}$ given by

$$
H_{\Lambda, \bar{h}}^{\omega}(\sigma)=-\sum_{i, j \in \Lambda} J_{i j} \sigma_{i} \sigma_{j}-\sum_{i \in \Lambda, j \notin \Lambda} J_{i j} \sigma_{i} \omega_{j}-\sum_{i \in \Lambda} h_{i} \sigma_{i}
$$

where $J_{i j} \geq 0$ for every $i, j \in G$, and $h_{i} \in \mathbb{R}$ for every $i \in G$. When $\bar{h} \equiv 0$, i.e., $h_{i}=0$ for every $i \in G$, we denote the Hamiltonian by $H_{\Lambda}^{\omega}:=H_{\Lambda, 0}^{\omega}$. Here, when we consider the ferromagnetic nearest-neighbor Ising model, we will assume $J_{i j}=J$ for some $J>0$ if $\mathrm{d}(i, j)=1$.

Since we will consider the product measure by $\prod_{i \in \Lambda} \nu\left(\sigma_{i}\right)=\prod_{i \in \Lambda}\left(\frac{1}{2} \delta_{+}+\frac{1}{2} \delta_{-}\right)$for every $i \in G$, given the inverse temperature $\beta>0$, the Gibbs measure of the Ising model in $\Lambda$ with boundary condition $\omega$ is the probability measure on $\Omega_{\Lambda}^{\omega}$ given by

$$
\mu_{\Lambda, \beta, \bar{h}}^{\omega}(\sigma)=\frac{1}{Z_{\Lambda, \beta, \bar{h}}^{\omega}} e^{-\beta H_{\Lambda, \bar{h}}^{\omega}(\sigma)}
$$

where $Z_{\Lambda, \beta, \bar{h}}^{\omega}$ is the partition function given by

$$
Z_{\Lambda, \beta, \bar{h}}^{\omega}=\sum_{\sigma \in \Omega_{\Lambda}^{\omega}} e^{-\beta H_{\Lambda, \bar{h}}^{\omega}(\sigma)} .
$$

When $\bar{h} \equiv 0$, then we denote $\mu_{\Lambda, \beta}^{\omega}:=\mu_{\Lambda, \beta, 0}^{\omega}$.

\subsubsection{On the lattice $\mathbb{Z}^{d}$}

For $d \geq 1$, define the set of configurations of spins in $\mathbb{Z}^{d}$ by $\Omega=\{-1,1\}^{\mathbb{Z}^{d}}$, and the distance of two vertices $i$ and $j$ in $\mathbb{Z}^{d}$ by $\mathrm{d}(i, j):=\|i-j\|_{1}$, where

$$
\|i\|_{i}=\sum_{k=1}^{d}\left|i_{k}\right|, \quad \text { with } i=\left(i_{1}, \ldots, i_{d}\right) \in \mathbb{Z}^{d} .
$$

The problem to show a phase transition for the ferromagnetic nearest-neighbor Ising model was raised by Lenz, who defined this model. Ising, when he was a Ph. D. student of Lenz, showed the absence of phase transitions at any temperature when considering the one-dimensional model. Afterwards, Peierls [135] showed that the model, with zero external fields undergoes a phase transition at low temperatures for $d \geq 2$, showing that $\mu_{\beta}^{+} \neq \mu_{\beta}^{-}$for sufficiently large $\beta$. Peierls invented a famous argument to show a phase transition, now called Peierls argument. This argument shows a bijection between the space of the spin configurations and the space of contour configurations when the boundary condition is prescribed to be plus or minus. For more explanation, see Section 5.5.1. For non-zero constant external fields $h_{i}=h$ with $h \neq 0$ for every $i \in \mathbb{Z}^{d}$, Lee and Yang [125] showed 
that, for any $d \geq 1$, the model has uniqueness at any temperature, and the proof uses results from complex analysis.

A translation by $j \in \mathbb{Z}^{d}$, denoted by $\theta_{j}: \mathbb{Z}^{d} \rightarrow \mathbb{Z}^{d}$, is defined by

$$
\theta_{j} i=i+j
$$

A probability measure $\mu \in \mathcal{M}_{1}(\Omega, \mathcal{F})$ is translation invariant if $\theta_{j} \mu=\mu$ for all $j \in \mathbb{Z}^{d}$.

For $\bar{h} \equiv 0$, Aizenman and Higuchi [2, 93] independently showed that every translation invariant Gibbs measure of the two-dimensional lattice ferromagnetic nearest-neighbor Ising model at inverse temperature $\beta$ is a convex combination of $\mu_{\beta}^{+}$and $\mu_{\beta}^{-}$, i.e., writing

$$
\left[\mu_{\beta}^{-}, \mu_{\beta}^{+}\right]=\left\{\mu: \text { There exists } \alpha \in[0,1] \text { such that } \mu=\alpha \mu_{\beta}^{+}+(1-\alpha) \mu_{\beta}^{-}\right\}
$$

we have

$$
\mathcal{G}_{\beta, 0}=\left[\mu_{\beta}^{-}, \mu_{\beta}^{+}\right] .
$$

The first proof of the existence of non-translation-invariant Gibbs measures on $\mathbb{Z}^{d}$ with $d \geq 3$ at sufficiantly low temperatures is due to Dobrushin [52]. Bodineau [26] showed that, for $d \geq 3$, every translation-invariant Gibbs measure of the $d$-dimensional lattice ferromagnetic short-range Ising model at inverse temperature $\beta$ is a convex combination of $\mu_{\beta}^{+}$and $\mu_{\beta}^{-}$. Raoufi [141] showed that for any inverse temperature $\beta$ and on any transitive amenable graph, the automorphism-invariant Gibbs states of the ferromagnetic Ising model are convex combinations of $\mu_{\beta}^{+}$and $\mu_{\beta}^{-}$. There is also a finite-volume version of the Aizenman and Higuchi Theorem for the two-dimensional Ising model by Coquille and Velenik [48].

There are several results for inhomogeneous external field, i.e., the external field $\bar{h}=\left(h_{i}\right)_{i \in \mathbb{Z}^{d}}$ is not constant. Bissacot and Cioletti [21] showed the following result.

Theorem 8. Let $d \geq 2$. If the external field $\bar{h}=\left(h_{i}\right)_{i \in \mathbb{Z}^{d}}$ satisfies

$$
\sum_{i \in \mathbb{Z}^{d}}\left|h_{i}\right|<\infty
$$

then the ferromagnetic Ising model undergoes a phase transition at low temperatures.

Theorem 8 claims that, if the external field $\bar{h}$ is weak enough in the sense that $\bar{h}$ is summable, then, different to Lee and Yang Theorem, the Ising model undergoes a phase transition at low temperatures. The proof is done by a Peierls argument. In [84], Chapter 7.4, we have a general result for general specifications and graphs.

Moreover, in [21] we also have a condition so that the model has uniqueness at any temperature.

Theorem 9. Let $d \geq 1$. If the external field $\bar{h}=\left(h_{i}\right)_{i \in \mathbb{Z}^{d}}$ with $h_{i}>0$ for every $i \in \mathbb{Z}^{d}$ satisfies $\lim \inf _{i \in \mathbb{Z}^{d}} h_{i}>0$, then the ferromagnetic Ising model has uniqueness at any temperature.

Although, for the physical point of view, Theorem 9 naturally holds since we already know from the Lee and Yang Theorem that the Ising model with homogeneous external field $h=\liminf _{i \in \mathbb{Z}^{d}} h_{i}$ has uniqueness at any temperature, the main point of Theorems 8 and 9 is the fact that the model is non-translation-invariant. Thus, many properties depending on translation-invariance are lost. The authors controlled the Radon-Nikodym derivative to deal with the non-invariant case.

For the case when $\bar{h}$ is non-summable, Bissacot, Cassandro, Cioletti and Presutti [20] showed the following result.

Theorem 10. Let $d \geq 2$ and $\bar{h}=\left(h_{i}\right)_{i \in \mathbb{Z}^{d}}$ be the external field given by

$$
h_{i}= \begin{cases}\frac{h^{*}}{\|i\|^{\gamma}}, & \text { if } i \neq 0, \\ h^{*}, & \text { if } i=0 .\end{cases}
$$


where $h^{*}>0$ and $\gamma>0$. Let us consider the ferromagnetic nearest-neighbor Ising model with external field $\bar{h}$. Then,

(1) If $\gamma>1$, then the model undergoes a phase transition at low temperatures.

(2) If $0<\gamma<1$, then the model has uniqueness at sufficiently low temperatures.

(3) If $\gamma=1$, then the model undergoes a phase transition at low temperatures if $h^{*}$ is small enough.

The proof of item (1) is by a Peierls argument, combined with an isoperimetric inequality. For item (2), they based themselves on an iterative scheme introduced in [29], showing that if there exists a thermodynamical limit of a sequence of finite-volume Gibbs measures, then this limit is equal to $\mu_{\beta}^{+}$. Since the model is absolutely summable, we know by Dobrushin's Uniqueness Theorem that the model has uniqueness at high temperatures. Thus, there is a gap in the temperature interval when $0<\gamma<1$. Afterwards, Cioletti and Vila [45], using Random Cluster representation (see [73, 91] for informations about this representation), closed the gap.

Theorem 11. Let $d \geq 2$ and $\bar{h}=\left(h_{i}\right)_{i \in \mathbb{Z}^{d}}$ be the external field given by (2.44). Then, for $0<$ $\gamma<1$, the ferromagnetic nearest-neighbor Ising model with external field $\bar{h}$ has uniqueness at any temperature.

There are several results for other types of external field. For instance, see [5, 6, 32, 33, 44, 99] for the random-field Ising model, [88, 133] for the alternating sign external field.

\subsubsection{On the Cayley Tree}

Let $\Gamma^{d}=(V, L)$ be the Cayley tree of order $d$, i.e., a $d+1$-regular infinite tree. Katsura and Takizawa developed the statistical mechanics of Cayley trees by using Bethe approximation. Afterwards, Preston showed the following result, when we consider the homogeneous external fields $h_{i}=h$ for every $i \in \Gamma^{d}$.

Theorem 12. Consider the ferromagnetic nearest-neighbor Ising model with parameter $J>0$ and $h \in \mathbb{R}$ on the Cayley tree $\Gamma^{d}$ of order $d \geq 2$. Let

$$
\beta(d):=\operatorname{arccoth} d=\frac{1}{2} \log \frac{d+1}{d-1}
$$

and

$$
h(\beta, d):= \begin{cases}0, & \text { if } \beta \leq \beta(d), \\ d \operatorname{arctanh}\left(\frac{d w-1}{d \bar{w}-1}\right)^{1 / 2}-\operatorname{arctanh}\left(\frac{d-\bar{w}}{d-w}\right)^{1 / 2}, & \text { if } \beta>\beta(d),\end{cases}
$$

where $\bar{w}=\operatorname{coth} \beta=w^{-1}$.

(a) If $\beta \leq \beta(d)$ or $|h|>h(\beta, d)$, then the model has uniqueness at $(\beta, h)$.

(b) In the opposite case, the model undergoes a phase transition at $(\beta, h)$.

Theorem 12 shows that the thorn-shaped region

$$
\left\{(\beta, h) \in \mathbb{R}^{2}: \beta>\beta(d),|h| \leq h(\beta, d)\right\}
$$

is the phase transition region of the ferromagnetic Ising model on the Cayley tree. Note that on Cayley trees, even in the presence of an external field of small absolute value, the model undergoes a phase transition, which differs from the lattice $\mathbb{Z}^{d}$. Preston showed that there is a large class of Gibbs measures that can be written as Markov chains. See Chapter 4 for more details.

Ganikhodjaev [83] showed that, if we consider an inhomogeneous external field, then the Ising model on Cayley tree of order $d \geq 2$ undergoes a phase transition at low temperatures when

$$
-J<\inf _{i \in \Gamma^{d}} h_{i}<\sup _{i \in \Gamma^{d}} h_{i}<J
$$




\subsubsection{Pressure and amenable graphs}

The pressure at finite volume $\Lambda \Subset G$ is defined by

$$
P_{\Lambda}^{\omega}(\beta, \bar{h})=\frac{1}{|\Lambda|} \log Z_{\Lambda, \beta, \bar{h}}^{\omega}
$$

The pressure is defined by

$$
P(\beta, \bar{h})=\lim _{\Lambda_{n} \Uparrow G} P_{\Lambda_{n}}^{\omega}(\beta, \bar{h}),
$$

where the sequence $\Lambda_{n} \Uparrow G$ converges o $G$ in the sense of van Hove, i.e., $\Lambda_{n} \uparrow G$ and

$$
\lim _{n \rightarrow \infty} \frac{\left|\partial^{\text {int }} \Lambda_{n}\right|}{\left|\Lambda_{n}\right|}=0
$$

where $\partial^{\text {int }} \Lambda:=\{i \in \Lambda$ : there exists $j \notin \Lambda$ such that $\langle j, i\rangle\}$. For example, on $\mathbb{Z}^{d}$, a sequence that satisfies this convergence is the hypercube $B(n)=\{-n, \ldots, n\}^{d}$.

By using Hölder inequality, we can show that the pressure at finite volume is convex. Moreover, the limit (2.51) exists, does not depend on the boundary condition, and $P$ is convex. The proof is the same as in [75], Lemma 3.5 and Theorem 3.6.

When $h_{i}=h$ for every $i \in G$, we write $P(\beta, \bar{h})=P(\beta, h)$. We have the following result that we can use to investigate uniqueness using the pressure on $\mathbb{Z}^{d}$.

Theorem 13. The following identities hold for every values $\beta>0$ and $h \in \mathbb{R}$.

$$
\frac{\partial P}{\partial h^{+}}(\beta, h)=\mu_{\beta, h}^{+}\left(\sigma_{0}\right) \quad \text { and } \quad \frac{\partial P}{\partial h^{-}}(\beta, h)=\mu_{\beta, h}^{-}\left(\sigma_{0}\right) .
$$

In particular, $h \mapsto P(\beta, h)$ is differentiable at $h$ if and only if there is uniqueness at $(\beta, h)$.

The Cheeger constant for the graph $G$ is defined by

$$
\kappa(G)=\inf _{\Lambda \Subset G} \frac{\left|\partial^{\text {int }} \Lambda\right|}{|\Lambda|} .
$$

If $\kappa(G)=0, G$ is said to be amenable and in case $\kappa(G)>0, G$ is said to be nonamenable.

An infinite graph $G$ is quasi-transitive if there exists a finite number of vertices $v_{1}, \ldots, v_{k}$ in $G$ such that for every $v \in G$, there is an automorphism of $G$ taking $v$ to some $v_{i}$. If $k=1$, the graph is transitive.

Jonasson and Steif [108] showed the presence or absence of a phase transition assuming some amenability properties of the graph for the ferromagnetic nearest-neighbor Ising model.

Theorem 14. Let $G$ be a graph with maximum degree $d<\infty$.

(a) If $G$ is nonamenable, $h>0$ and $\beta>(2 \kappa(G))^{-1}(2 h+1+\log (3(d+1)))$, then the model undergoes a phase transition at $(\beta, h)$.

(b) If $G$ is amenable and quasi-transitive, then the Ising model on $G$ has uniqueness at any $(\beta, h)$ with $h>0$ and $\beta>0$.

Given a positive external field $\bar{h}=\left(h_{i}\right)_{i \in G}$, i.e., $h_{i}>0$ for every $i \in G$, we say that $\bar{h}$ decays to zero at infinity if, for every $\varepsilon>0$, there exists a finite set $\Lambda_{\varepsilon} \Subset G$ such that

$$
h_{i}<\varepsilon, \quad \text { for every } i \notin \Lambda_{\varepsilon} \text {. }
$$

From [20], we know that any system where the external field decays to zero at infinity has the same pressure function as the system without external field. Let us show this result. 
Proposition 1. Consider the ferromagnetic nearest-neighbor Ising model. If $G$ is amenable and $\bar{h}$ decays to zero at infinity, then

$$
P(\beta, \bar{h})=P(\beta, 0)
$$

Proof. For a fixed finite volume $\Lambda$ and boundary condition $\omega$,

$$
\left|P_{\Lambda}^{\omega}(\beta, \bar{h})-P_{\Lambda}^{\omega}(\beta, 0)\right| \leq \frac{\beta}{|\Lambda|}\left\|H_{\Lambda, \bar{h}}^{\omega}-H_{\Lambda}^{\omega}\right\|_{\infty} .
$$

Since we have

$$
\begin{aligned}
\left\|H_{\Lambda, \bar{h}}^{\omega}-H_{\Lambda}^{\omega}\right\|_{\infty} & =\sup _{\sigma \in \Omega}\left|H_{\Lambda, \bar{h}}^{\omega}(\sigma)-H_{\Lambda}^{\omega}(\sigma)\right| \\
& \leq 2 J\left|\partial^{\text {int }} \Lambda\right|+\sup _{\sigma \in \Omega}\left|\sum_{i \in \Lambda} h_{i} \sigma_{i}\right|,
\end{aligned}
$$

and $\bar{h}$ decays to zero at infinity, given $\varepsilon>0$, there exists $\Lambda_{\varepsilon} \Subset G$ such that $h_{i}<\varepsilon / 3$ for every $i \notin \Lambda_{\varepsilon}$. Choose a finite set $\Lambda_{0} \supset \Lambda_{\varepsilon}$ large enough such that

$$
\begin{aligned}
\frac{1}{\left|\Lambda_{0}\right|} \sum_{i \in \Lambda_{0}} h_{i} & =\frac{1}{\left|\Lambda_{0}\right|} \sum_{i \in \Lambda_{\varepsilon}} h_{i}+\frac{1}{\left|\Lambda_{0}\right|} \sum_{i \in \Lambda_{0} \backslash \Lambda_{\varepsilon}} h_{i} \\
& \leq \frac{1}{\left|\Lambda_{0}\right|} \sum_{i \in \Lambda_{\varepsilon}} h_{i}+\frac{\varepsilon}{3} \\
& <\frac{\varepsilon}{2} .
\end{aligned}
$$

Since $G$ is amenable, we choose $\Lambda_{0}$ large enough such that

$$
\frac{\left|\partial^{\text {int }} \Lambda_{0}\right|}{\left|\Lambda_{0}\right|}<\frac{\varepsilon}{4 J}
$$

as desired.

\subsection{Dyson model}

Let us consider the one-dimensional lattice $\mathbb{Z}$, and let $\Omega=\{-1,1\}^{\mathbb{Z}}$ be the set of configurations $\sigma=\left(\sigma_{i}\right)_{i \in \mathbb{Z}}$ on $\mathbb{Z}$. The Hamiltonian of the Dyson model in a finite volume $\Lambda$ with boundary condition $\omega$ is defined by

$$
H_{\Lambda, \bar{h}}^{\omega}(\sigma)=-\sum_{\substack{i, j \in \Lambda \\ i \neq j}} J_{i j} \sigma_{i} \sigma_{j}-\sum_{\substack{i \in \Lambda \\ j \notin \Lambda}} J_{i j} \sigma_{i} \omega_{j}-\sum_{i \in \Lambda} h_{i} \sigma_{i},
$$

where $h_{i} \in \mathbb{R}$ for every $i \in \mathbb{Z}$, and the coupling constants $J_{i j}$ are defined by

$$
J_{i j}= \begin{cases}J & \text { if }|i-j|=1, \\ |i-j|^{-\alpha} & \text { if }|i-j|>1,\end{cases}
$$

where $J(1)=J>0$ and $1<\alpha \leq 2$.

For an inverse temperature $\beta>0$, the Gibbs measure of the Dyson model in $\Lambda \subset \mathbb{Z}$ with boundary condition $\omega$ is given by the same expression (2.37), replacing the Hamiltonian by $H_{\Lambda}^{\omega}$. Since the FKG inequality holds for the Dyson model, there exists the infinite-volume Gibbs measure $\mu_{\Lambda, \beta}^{+}$(resp. $\mu_{\Lambda, \beta}^{-}$) with plus (resp. minus) boundary condition. Moreover, Corollary 1 and Theorem 7 hold for the Dyson model. 
It is well known that for $\alpha>2$ there is no phase transition at any temperature $[50,51,53,78$, 152]. Dyson showed in [54] via comparison with a hierarchical model, that, for $\alpha \in(1,2)$, the model undergoes a phase transition at low temperatures. Afterwards, Fröhlich and Spencer [77] showed the existence of a phase transition for $\alpha=2$. The proof of these authors was done by a contour argument; they invented a notion of one-dimensional contours on $\mathbb{Z}$ in order to prove the phase transition. Their strategy more or less followed the classical Peierls contour argument used for the standard nearest-neighbor Ising model, but with a substantially more sophisticated definition of contours. Phase transitions for smaller $\alpha \in(1,2)$ can then be deduced by Griffiths inequalities for low enough temperature.

Afterwards different proofs were invented to show the transition. One of them used Reflection Positivity [76]. The method of infrared bounds offers an alternative to obtain bounds on contour probabilities. In fact, the authors of [76] remark that they can cover a general class of long-range one-dimensional pair interactions including the ones treated in [54].

Another way to derive it was a comparison with independent percolation via Fortuin inequalities and Griffiths inequalities for the $\alpha=2$ case, as discussed in [3].

Cassandro et al. in [39] rigorously formalized the contour argument of [77] in the parameter regime $\alpha_{+}<\alpha \leq 2$, where $\alpha_{+}:=3-\log 3 / \log 2 \approx 1.4150$. The construction allows a more precise description of various properties of the model. It has been used in various follow-up papers $[23,40,41,42,43,126,127]$. We should emphasize that, although the use of contour arguments may look somewhat unwieldy in comparison with other approaches, it is much more robust. Indeed it has been used to analyse Dyson models in random [42, 43] and periodic fields [115], for interface behaviour and phase separation [40,41], for entropic repulsion [23], and [22] for the model in decaying magnetic fields, all problems where alternative methods appear to break down.

Let us briefly present the results in [22]. Cassandro et al. in [39] showed the phase transition at low temperatures in the parameter regime $\alpha_{+}<\alpha \leq 2$ and supposing that $J(1) \gg 1$. We showed that, reducing the regime of the parameter $\alpha$ to $\alpha_{*}<\alpha \leq 2$, where $\alpha_{*} \approx 1.729$ is defined by

$$
\sum_{n=1}^{\infty} \frac{1}{n^{\alpha_{*}}}=2,
$$

the model undergoes a phase transition at low temperatures, but assuming $J(1)=1$. Using [127], we can extend to any $\alpha \in(1,2]$ by using the quasi-additive property of the Hamiltonian.

Adding the external fields $\bar{h}=\left(h_{i}\right)_{i \in \mathbb{Z}}$ given by

$$
h_{i}=\frac{h^{*}}{(1+|i|)^{\gamma}},
$$

where $h^{*}>0$ and $\gamma>0$, we have the following result.

Theorem 15. Let $\alpha^{*}$ defined in (2.60). Consider the Dyson model on $\mathbb{Z}$ such that $\bar{h}=\left(h_{i}\right)_{i \in \mathbb{Z}}$ are defined in (2.61). We assume either

- $\alpha \in(1,2], J(1)=1$ and $\gamma>\max \left\{\alpha-1, \alpha^{*}-1\right\}$, or

- $\alpha \in\left(\alpha^{*}, 2\right], J(1)=1, \gamma=\alpha-1$ and $h^{*}$ small enough

Then there exists $\beta_{c}>0$ such that for all $\beta>\beta_{c}$ the model undergoes a phase transition.

The proof uses a Peierls argument where the contours are the same as in [39]. The heuristic argument is the following. The total energy between inside and outside of a finite interval $\Lambda$ is obtained by summing the coupling constants over pairs of vertices such that one vertex is inside and one outside the interval.

$$
\sum_{\substack{i \in \Lambda \\ j \notin \Lambda}} \frac{1}{|i-j|^{\alpha}}=O\left(|\Lambda|^{2-\alpha}\right) .
$$


On the other hand, the energy of the external field on $\Lambda$ is

$$
\sum_{i \in \Lambda} \frac{1}{|i|^{\gamma}}=O\left(|\Lambda|^{1-\gamma}\right) .
$$

Thus, if $2-\alpha>1-\gamma$, the model should undergo a phase transition. In the case $2-\alpha=1-\gamma$, we should compare the constants of the energy, and to have the phase transition, the constant of the energy (2.63) should be smaller than the constant of the energy (2.62). This is the reason why $h^{*}$ should be small enough. When $2-\alpha<1-\gamma$, we conjecture that the model has uniqueness at any temperature. We also conjecture that the model undergoes a phase transition at low temperatures for every $\alpha \in(1,2]$ and $\gamma>\alpha-1$. 



\section{Chapter 3}

\section{Entropic repulsion and lack of the $g$-measure property for Dyson models}

\subsection{Introduction}

Dyson models, long-range Ising models with ferromagnetic, polynomially decaying, pair interactions, have been studied for a considerable time. After Dyson [54, 55] proved the existence of a phase transition, confirming a conjecture due to Kac and Thompson [109], various alternative proofs and further properties have been derived. One recent low-temperature result which we will find particularly useful is the existence of phase separation, properly defined, with an "interface point", which is to some extent stable under infinite-volume limits with appropriate mixed boundary conditions similar to Dobrushin boundary conditions introduced in higher dimensions. Indeed, in [41] it was shown that a Dyson model in a finite interval of length L, with --boundary conditions on the left and +-boundary conditions on the right, has an interface of "mesoscopic size" for decay parameter values ${ }^{1} \alpha_{+}<\alpha<2$, once the temperature is low enough (but non-zero). This means that with overwhelming probability its location is in the middle of the interval, up to a Gaussian correction which grows sublinearly with $L$.

In this chapter we notice that this interface result implies in a fairly straightforward manner that a form of entropic repulsion occurs, in the sense that a large interval of minuses inserted in the +phase has two moderately large intervals around $i^{2}{ }^{2}$ in which the system will be in the --phase. We use this observation to show that the low-temperature Gibbs measures of the Dyson model are not $g$-measures: their conditional probabilities w.r.t. the past are not necessarily continuous functions of this past. It was shown before that there exist $g$-measures which are not Gibbs measures [62]; our result answers a question raised in [63] and shows that neither class of measures contains the other one. Although the question had been posed before, it seems to be the case that there were no precise conjectures whether these Dyson Gibbs measures actually were $g$-measures or not. We thus elucidate a somewhat unclear situation, about the connection between two similar-looking notions, originating in two different fields of research (namely Mathematical Statistical Mechanics and Dynamical Systems).

Warning: The case $\alpha=2$ is somewhat different; as the fluctuations in the location of the interface are macroscopic, rather than mesoscopic [41], our arguments do not fully work in that case. We also note that the proof(s) and even the properties of the phase transition for this borderline case had already required a special treatment before. The model gives rise to a more complex situation in which an intermediate phase arises [98], and also a discontinuity of the critical magnetisation occurs [3].

\footnotetext{
${ }^{1}$ Our results will be valid only for $\alpha$ satisfying the lower bound $\alpha>\alpha_{+}-$already present in [39, 41, 42]. In contrast to the upper bound $\alpha<2$, we believe this lower bound is technical only, as we shall see.

${ }^{2}$ They are the "wet" regions, while the frozen interval is a hard wall in a "complete wetting" situation.
} 


\subsection{Definitions and Notations}

\subsection{1 $g$-measures}

Let $S$ be a finite, countable or compact set. Here, we consider $S$ be a finite set, e.g. $S=\{0,1\}$. Let $m$ and $n$ be integer numbers with $m \leq n$, and the set

$$
S_{m}^{n}=\left\{\left(x_{k}\right)_{m \leq k \leq n}: x_{k} \in S\right\} .
$$

We denote by $x_{m}^{n}$ be the finite sequence $\left(x_{m}, \ldots, x_{n}\right)$. We also consider $n=\infty$, where $x_{m}^{\infty}$ is the infinite sequence $\left(x_{m}, x_{m+1}, \ldots\right)$, and $m=-\infty$, where $x_{-\infty}^{n}$ is the sequence $\left(\ldots, x_{n-1}, x_{n}\right)$. The set $S_{-\infty}^{0}$ is metrizable with the following metric: for two sequences $x, y \in S_{-\infty}^{0}$,

$$
\mathrm{d}(x, y)=2^{-\sup \left\{n \geq 0: x_{k}=y_{k},-n \leq k \leq 0\right\}} .
$$

Let $T: S_{-\infty}^{0} \rightarrow S_{-\infty}^{0}$ be the shift defined by $(T x)_{n}=x_{n-1}$. We denote by $\mathcal{P}=\mathcal{P}_{S_{-\infty}^{0}}$ the class of continuous positive functions $g: S_{-\infty}^{0} \rightarrow[0,1]$ such that

$$
\sum_{y \in T^{-1} x} g(y)=1, \quad \text { for all } x \in S_{-\infty}^{0} .
$$

These functions are called $g$-functions.

For a fixed set $\Omega$, a function $f: \Omega \rightarrow \mathbb{R}$ is called strongly non-null if

$$
\inf _{x \in \Omega} f(x)>0 .
$$

Note that, in our case, since $S$ is finite and $g$-functions are continuous, every $g$-function is strongly non-null.

Fernández, Gallo and Maillard called regular g-function a continuous probability kernel $P$ : $S \times S_{-\infty}^{-1}$ satisfying

$$
\sum_{x_{0} \in S} P\left(x_{0} \mid x_{-\infty}^{-1}\right)=1, \quad \text { for all } x_{-\infty}^{-1} \in S_{-\infty}^{-1} .
$$

Note that the conditions (3.3) and (3.5) are equivalent.

Let $\mathcal{F}$ be the $\sigma$-algebra generated by cylinder sets

$$
\left[c_{-n}^{0}\right]=\left\{x_{-\infty}^{0} \in S_{-\infty}^{0}: x_{k}=c_{k},-n \leq k \leq 0\right\},
$$

for every $c_{-n}^{0} \in S_{-n}^{0}$. For a fixed $g$-function $g$, a probability measure $\mu$ on $S_{-\infty}^{0}$, with $\sigma$-algebra $\mathcal{F}$ is called a $g$-measure if, for any continuous function $f: S_{-\infty}^{0} \rightarrow \mathbb{R}$,

$$
\int_{S_{-\infty}^{0}} f(x) \mu(\mathrm{d} x)=\int_{S_{-\infty}^{0}}\left[\sum_{y \in T^{-1} x} f(y) g(y)\right] \mu(\mathrm{d} x) .
$$

Note that $\mu$ is shift-invariant.

As discussed in $[63,64]$, which discuss a lot of the history, the terminology " $g$-measures" was introduced by Keane [112], but the notion is older. In those papers also the observation is made and exploited that the $g$-measure property is a kind of one-sided Gibbs property. However, this analogy appears to work properly mostly in various uniqueness regimes, as we illustrate here.

There are definitions less restrictive, for instance, assuming mensurability instead of continuity, removing the translation invariance (in that case the $g$-measure is called $G$-measure). See [35, 36, 80, 106, 112]. The complete formalism - providing all conditional probabilities w.r.t. to the past can be restored under extra conditions via the notion of a "Left Interval Specification" (LIS) [63, 64].

Note that $g$-measures are extensions of (one-sided) Markov properties, which have been studied 
under different names in various areas of mathematics for a long time, such as Chains with infinite connections [16], Chains of infinite order [92], Variable Length Markov Chains [145], uniform martingales [110] etc. For a number of papers addressing $g$-measures and related properties, see e.g. $[17,18,31,35,36,49,74,79,80,81,96,97,105,106,167]$. When the interactions are finite-range, $g$-measures are Markov chains.

Note that, by shift-invariance, the $g$-measure $\mu$ can be extended in a unique way to $\left(S^{\mathbb{Z}}, \mathcal{F}\right)$, where $S^{\mathbb{Z}}$ is the set of sequences of $S$ indexed by $\mathbb{Z}$,

$$
S^{\mathbb{Z}}=\left\{\left(x_{n}\right)_{n \in \mathbb{Z}}: x_{n} \in S\right\} .
$$

In fact, note that the $\mu_{n}=\mu$ is also a $g$-measure over $\left(S_{-\infty}^{n}, \mathcal{F}\right)$, since $S_{-\infty}^{n} \cong S_{-\infty}^{0}$.

For each $g$-function $g$, define the Ruelle operator by

$$
\left(L_{g} h\right)(x)=\sum_{y \in T^{-1} x} g(y) h(y)
$$

for all continuous functions $h$ on $S_{-\infty}^{0}$. The dual of $L_{g}$, denoted by $L_{g}^{*}$, is defined on the set of probability measures by

$$
L_{g}^{*} \mu(h)=\mu\left(L_{g} h\right)
$$

for all probability measures on $S_{-\infty}^{0}$ and all continuous functions $h$ on $S_{-\infty}^{0}$. The following result, due to Ledrappier [124], shows some equivalences for $g$-measures.

Theorem 16. Let $g \in \mathcal{P}$ be a $g$-function, and $\mu$ be a probability measure on $S_{-\infty}^{0}$. The following statements are equivalent:

1. $\mu$ is a g-measure.

2. For every $a \in S$,

$$
\mathbb{E}_{\mu}\left[\mathbb{1}_{[a]} \mid T^{-1} \mathcal{F}\right](x)=g\left(x_{-\infty}^{-1} a\right) \quad \text { н-a.e. } x \in S_{-\infty}^{0},
$$

where $[a]$ is the cylinder $[a]=\left\{x_{-\infty}^{0} \in S_{-\infty}^{0}: x_{0}=a\right\}$.

3. $L_{g}^{*} \mu=\mu$.

Palmer, Parry and Walters [134] showed the following equivalence for $g$-measures.

Theorem 17. A fully supported measure $\mu$ on $S_{-\infty}^{0}$ is a g-measure if and only if the sequence

$$
\mu\left(x_{0} \mid x_{-n}^{-1}\right):=\frac{\mu\left(\left[x_{-n}^{0}\right]\right)}{\mu\left(\left[x_{-n}^{-1}\right]\right)}
$$

converges uniformly as $n \rightarrow \infty$.

We define the $n$-th variation of $g \in \mathcal{P}$ by

$$
\operatorname{var}_{n}(g):=\sup _{\substack{x, y \in S_{-\infty}^{0} \\ x_{k}=y_{k},-n \leq k \leq-1}}|g(x)-g(y)| .
$$

Note that, since a $g$-function $g \in \mathcal{P}$ is continuous in $S_{-\infty}^{0}$ and hence $g$ is uniformly continuous (since $S$ is finite), the $n$-th variation of $g$ converges to zero as $n \rightarrow \infty$. We said that $g \in \mathcal{P}$ has summable variation if

$$
\sum_{n=1}^{\infty} \operatorname{var}_{n}(g)<\infty
$$

Walters [168] showed that every summable variation $g$-function admit an unique $g$-measure. There are a lot of results concerning the problem to find conditions for $g$-functions in order to admit 
an unique $g$-measure. See $[16,92,104,121,165]$ when the alphabet is finite, and $[46,112,129,168]$ when the alphabet is countable or compact.

Theorem 18. Suppose $g \in \mathcal{P}$ has summable variation, then $g$ admits an unique $g$-measure.

On the other hand, the problem to find an example of a $g$-function that admits more than one $g$-measure was first solved by Bramson and Kalikow [31]. We will present this $g$-function here. Consider $S=\{-1,1\}$ and let $\left(m_{j}\right)_{j \geq 1}$ be an increasing sequence of odd positive integers. Let $\left(p_{j}\right)_{j \geq 1}$ be an decreasing sequence of positive real numbers satisfying

$$
\sum_{j=1}^{\infty} p_{j}=1 \quad \text { and } \quad p_{k} \leq \frac{1}{2} \sum_{j>k} p_{j} \quad \text { for every } k .
$$

Let $\mu$ be the probability measure supported by $\left(m_{j}\right)_{j \geq 1}$ defined by $\mu\left(m_{j}\right)=p_{j}$. For $x \in S_{-\infty}^{0}$ and $\varepsilon \in\left(0, \frac{1}{2}\right)$, define

$$
W\left(x, m_{j}\right)= \begin{cases}1-\varepsilon, & \text { if } x_{0} \cdot \sum_{i=1}^{m_{j}} x_{-i}>0, \\ \varepsilon, & \text { if } x_{0} \cdot \sum_{i=1}^{m_{j}} x_{-i}<0 .\end{cases}
$$

Define the $g$-function by the following prescription,

$$
g(x)=\sum_{j=1}^{\infty} W\left(x, m_{j}\right) p_{j} .
$$

Theorem 19. The $g$-function $g$ defined in (3.17) is continuous and $g$ admits two extremal $g$ measures.

\subsubsection{One-dimensional Gibbsian specification}

For a fixed configuration $\sigma \in S^{\mathbb{Z}}$ and $m, n$ integers with $m \leq n$, we keep the notation $\sigma_{m}^{n} \in S_{m}^{n}$ for the finite sequence $\left(\sigma_{m}, \ldots, \sigma_{n}\right)$.

For fixed $m, n \in \mathbb{Z}$ with $m \leq n$ and $\sigma_{k} \in S$ for $m \leq k \leq n$, we will denote the cylinder by

$$
\left[\sigma_{m}^{n}\right]:=C_{m, \ldots, n}^{\sigma_{m}, \ldots, \sigma_{n}}=\left\{\omega \in S^{\mathbb{Z}}: \omega_{k}=\sigma_{k}, m \leq k \leq n\right\} .
$$

Here, the specification is a family of continuous probability kernels $\gamma_{\Lambda_{L}}$ with $L \in \mathbb{N}$, where $\Lambda_{L}=$ $[-L, L]$ is the set $\{-L, \ldots, L\}$, which prescribes its conditional probabilities jointly w.r.t. the past and future via

$$
\gamma_{\Lambda_{L}}\left(\left[\sigma_{-L}^{L}\right] \mid \omega_{\Lambda_{L}^{c}}\right):=\mathbb{E}_{\mu}\left(\left[\sigma_{-L}^{L}\right] \mid \mathcal{F}_{\Lambda_{L}^{c}}\right)(\omega) \quad \mu \text { - a.e. } \omega,
$$

where $\mu$ is a Gibbs measure. Thanks to their quasilocality properties, Gibbs measures are the non-null measures for which the $\gamma_{\Lambda_{L}}$ are continuous functions of $\omega$. In this case, it is possible to reconstruct all the conditional probabilities (3.19) from the single-site conditional probabilities at time $i \in \mathbb{Z}$, given for $\mu$ a.e. $\omega$ by

$$
\gamma_{i}(\omega):=\mathbb{E}_{\mu}\left(\left[\sigma_{i}\right] \mid \mathcal{F}_{\{i\}^{c}}\right)(\omega)
$$

or, more shortly

$$
\gamma_{i}(\omega):=\mu\left(\left[\sigma_{i}\right] \mid \mathcal{F}_{\{i\}^{c}}\right)(\omega),
$$

where $\left[\sigma_{i}\right]=C_{i}^{\sigma_{i}}$. Since the model is shift-invariant, it is enough to consider

$$
\gamma_{0}(\omega):=\mu\left(\left[\sigma_{0}\right] \mid \mathcal{F}_{\{0\}^{c}}\right)(\omega),
$$

We shall encounter later the past and future $\sigma$-algebras $\mathcal{F}_{<0}$ and $\mathcal{F}_{>0}$ generated by the projections indexed by negative and positive integers. The function $\gamma_{0}$ is a $\mathcal{F}_{\{0\}}$-measurable function and when the measure is a Gibbs measure, this function is continuous, jointly in past and future. 
Definition 4. A specification $\gamma$ is regular if, for every $i \in \mathbb{Z}$,

1. the function $\gamma_{i}\left(\left[\sigma_{i}\right] \mid \cdot\right)$ is continuous for every $\sigma_{i} \in S$, i.e., for every $\varepsilon>0$, there exists $n, m \geq 0$ such that

$$
\left|\gamma_{i}\left(\left[\sigma_{i}\right] \mid \omega_{\{i\}^{c}}\right)-\gamma_{i}\left(\left[\sigma_{i}\right] \mid \eta_{\{i\}^{c}}\right)\right|<\varepsilon,
$$

for every $\omega, \eta \in S^{\mathbb{Z}}$ with $\omega_{k}=\eta_{k}$, for $-m \leq k \leq n$.

2. the function $\gamma_{i}\left(\left[\sigma_{i}\right] \mid \cdot\right)$ is strongly non-null for every $\sigma_{i} \in S$, i.e.,

$$
\inf _{\omega \in S^{\mathbb{Z}}} \gamma_{i}\left(\left[\sigma_{i}\right] \mid \omega_{\{i\}^{c}}\right)>0
$$

The following result is the Gibbs measure's version of Theorem 17. See [18] for the proof.

Theorem 20. A shift-invariant probability measure $\mu$ on $\left(S^{\mathbb{Z}}, \mathcal{F}\right)$ is a Gibbs measure if and only if $\mu$ is non-null and the sequence

$$
\mu\left(\left[\sigma_{0}\right] \mid \sigma_{-n}^{-1}, \sigma_{1}^{m}\right):=\frac{\mu\left(\left[\sigma_{-n}^{m}\right]\right)}{\mu\left(\left[\sigma_{-n}^{1}\right] \cup\left[\sigma_{1}^{m}\right]\right)}
$$

converges uniformly as $n, m \rightarrow \infty$.

Kozlov [118] shows a characterization when a specification is Gibbsian.

Theorem 21. A specification is Gibbsian if, and only if, it is strongly non-null and quasilocal.

Remember that, when the state space $S$ is compact and discrete, quasilocal functions and continuous functions are equivalent.

In our one-dimensional setting, a basis of neighborhoods for a configuration $\omega$ in the configuration space $\Omega:=\{-1,+1\}^{\mathbb{Z}}$ can be chosen of the form

$$
\mathcal{N}_{L}(\omega):=\left\{\sigma \in \Omega: \sigma_{\Lambda_{L}}=\omega_{\Lambda_{L}}\right\}, L \in \mathbb{N}
$$

where $\omega_{\Lambda_{L}}$ is the restriction of $\omega$ to the sites in $\Lambda_{L}$. For any integers $N>L$, we shall also consider particular open subsets of neighborhoods $\mathcal{N}_{N, L}^{+}(\omega)\left(\operatorname{resp} . \mathcal{N}_{N, L}^{-}(\omega)\right)$ on which the configuration is + (resp. -) on the annulus $\Lambda_{N} \backslash \Lambda_{L}$ for $N>L$ :

$$
\mathcal{N}_{N, L}^{+}(\omega):=\left\{\sigma \in \mathcal{N}_{L}(\omega): \sigma_{\Lambda_{N} \backslash \Lambda_{L}}=+_{\Lambda_{N} \backslash \Lambda_{L}}\right\}\left(\operatorname{resp} . \mathcal{N}_{N, L}^{-}(\omega)\right)
$$

where for $\Lambda \subset \mathbb{Z},+_{\Lambda}$ is the configuration in $\Lambda$ in which all the spins are plus. Similarly we define the one-sided equivalent objects, such as $\mathcal{N}_{N, L}^{+, \text {left }}(\omega)\left(\operatorname{resp} . \mathcal{N}_{N, L}^{-, \text {left }}(\omega)\right)$ when the $N$ spins to the left of the interval $\Lambda_{L}$ are constrained to be plus (resp. minus).

$$
\begin{aligned}
\mathcal{N}_{L}^{\text {left }}(\omega) & :=\left\{\sigma \in \Omega: \sigma_{k}=\omega_{k},-L \leq k<0\right\}, L \in \mathbb{N} . \\
\mathcal{N}_{N, L}^{+, \text {left }}(\omega) & :=\left\{\sigma \in \mathcal{N}_{L}^{\text {left }}(\omega): \sigma_{k}=+1,-N \leq k<-L\right\} .
\end{aligned}
$$

Considering the lattice $\mathbb{Z}$ as a bi-infinite sequence of "times", it is tempting to consider measures on $\Omega$ as stochastic processes (and to transfer the Gibbs property to some Markovian-like or almostMarkovian property). This equivalence holds in particular under conditions of weak coupling, such as when a Dobrushin uniqueness condition holds, for example for long-range Dyson models at high temperature, as well as for short-range models in which the coupling between two infinite half-lines is uniformly bounded. In the latter case the equivalence holds at all temperatures. However, it is far from obvious if such a description is always easily possible (see e.g. [62, 63, 64]). In fact, the non-equivalence between one-sided and two-sided conditionings, which we will demonstrate in detail later, serves as a warning to a too easy identification. 


\subsubsection{Not every $g$-measure is a Gibbs measure}

When the interactions are short-range, i.e., there exists $k \geq 1$ such that $P\left(x_{0} \mid x_{-\infty}^{-1}\right)=P\left(x_{0} \mid x_{-k}^{-1}\right)$ for every $x_{0} \in S$ and $x_{-\infty}^{-1} \in S_{\infty}^{-1}, g$-measures are Markov chains. These coincide with Gibbs measures, which then are Markov fields, expressible in two-sided conditional probabilities, see e.g. [84], Chapter 3. In fact, this equivalence applies for a large class of interactions which satisfy a strong uniqueness condition [63,64]. In the case when the range of the interactions of the $g$-measure are infinite, we need some regularity of the potentials, i.e., potentials that have a sufficiently fast decay, such as summable variation, to imply that the $g$-measures are Gibbs. See [63] for more informations. Berghout, Fernández and Verbitskiy [18] showed a characterization of the Gibbs measures assuming that the measure is a $g$-measure. They also discussed when Gibbs measures are $g$-measures.

Theorem 22. Let $\mu$ be a g-measure. Then $\mu$ is Gibbs if and only if the sequences of functions $\left\{f_{n}^{\sigma_{0}, \eta_{0}}\right\}_{n \geq 1}$, given by

$$
f_{n}^{\sigma_{0}, \eta_{0}}(\omega)=\prod_{i=-n}^{-1} \frac{g\left(\omega_{i}^{-1} \sigma_{0} \omega_{1}^{\infty}\right)}{g\left(\omega_{i}^{-1} \eta_{0} \omega_{1}^{\infty}\right)}
$$

converges, for all $\sigma_{0}, \eta_{0} \in\{-1,1\}$, uniformly in $\omega \in\{-1,1\}^{\mathbb{Z}}$, as $n \rightarrow \infty$.

See also [19] for the proof that the Schonmann projection is almost surely a regular $g$-measure.

Fernández, Gallo and Maillard [62] showed that there exists $g$-measures that are not Gibbs measures. This mean that we need more information than continuity of the $g$-function to conclude that the class of $g$-measures is contained in the class of Gibbs measures.

Definition 5. Let $g$ be a measurable function and $\mu$ a probability measure on $(\Omega, \mathcal{F})$. Let $\omega \in \Omega$. We say that $g$ is $\mu$-essentially discontinuous at $\omega$ if every function continuous at $\omega$ differs from $g$ in a set of non-zero $\mu$-measure.

Consider a converging sequence $\left\{p_{i}\right\}_{i \geq 0}$ with $p_{i} \in(0,1)$ satisfying:

$$
\inf _{i \geq 0} p_{i}=\varepsilon>0 \quad \text { and } \quad p_{\infty}=\lim _{i \rightarrow \infty} p_{i} .
$$

For $\omega \in\{-1,1\}^{\mathbb{Z}}$, consider

$$
\ell\left(\omega_{-\infty}^{-1}\right)=\min \left\{j \geq 0: \omega_{-j-1}=1\right\} .
$$

Define

$$
g\left(\omega_{-\infty}^{-1} 1\right)=p_{\ell\left(\omega_{-\infty}^{-1}\right)}
$$

Theorem 23. There exists choices of the sequences $\left\{p_{i}\right\}_{i \geq 0}$ satisfying (3.29) for which

$$
\mu\left(\omega_{0}=-1 \mid \cdot\right) \text { is essentially discontinuous at }-1_{-\infty}^{+\infty},
$$

where $\mu$ is the (unique and non-null) $g$-measure compatible with the $g$-function defined in (3.31).

Theorem 21 implies that this $g$-measure is not a Gibbs measure.

\subsubsection{Gibbs vs $g$-measures for Dyson models in the Phase Transition region}

In general, where the interactions of the $g$-measure are necessarily long-range, there is not that much known in the phase transition region. Phase transitions in the Gibbs measure context have been known to occur since Dyson, and in the $g$-measure context they are also known to be possible [17, 31, 49, 74, 97]. Nevertheless, there seems little known about the equivalence of the Gibbs measure property and the $g$-measure property in any such general context. 
Let $G$ be an infinite, locally finite and connected graph. A probability measure $\mu$ on $S^{G}$ has the local Markov property such that, for each finite set $\Lambda \Subset G$,

$$
\mu\left(F \mid \mathcal{F}_{\mathbb{Z}^{d} \backslash \Lambda}\right)=\mu\left(F \mid \mathcal{F}_{\partial \Lambda}\right),
$$

for every $\Lambda$-measurable $F$. A probability measure $\mu$ has the global Markov property (see e.g. [70]) if (3.33) holds for arbitrary (not necessary finite) subset $\Lambda$ of $G$. In higher dimension, one could interpret the local Markov property as a Gibbs property, and the global Markov property to some extent as the equivalent of the $g$-measure property. It is known that there are measures having the local, but not the global Markov property [87, 102, 169]. Here we will show the somewhat analogous result that the Gibbs measures of the Dyson model are not $g$-measures.

We consider configurations lying in the probability space $(\Omega, \mathcal{F}, \rho)=\left(S, \mathcal{S}, \rho_{0}\right)^{\mathbb{Z}}$ where $S=$ $\{-1,+1\}$ is equipped with the a priori product measure

$$
\rho_{0}=\frac{1}{2} \delta_{-1}+\frac{1}{2} \delta_{+1}
$$

For a configuration $\omega \in \Omega$ and any $\Lambda \subset \mathbb{Z}$, we consider the restriction $\omega_{\Lambda}$ and the corresponding configuration spaces at volume $\Lambda$ as the product probability spaces $\left(\Omega_{\Lambda}, \mathcal{F}_{\Lambda}, \rho_{\Lambda}\right)$ defined in a standard way. The Hamiltonian of the Dyson model is given by

$$
H_{\Lambda}^{\omega}(\sigma)=-\sum_{\substack{i, j \in \Lambda \\ i \neq j}} J_{i j} \sigma_{i} \sigma_{j}-\sum_{\substack{i \in \Lambda \\ j \notin \Lambda}} J_{i j} \sigma_{i} \omega_{j},
$$

where the coupling constants $J_{i j}$ are defined by

$$
J_{i j}= \begin{cases}J(1) & \text { if }|i-j|=1, \\ |i-j|^{-\alpha} & \text { if }|i-j|>1,\end{cases}
$$

where $J(1)>0$ and $1<\alpha \leq 2$.

To specify the two-sided conditional probabilities of our Dyson measures, we consider the set $\mathscr{L}$ of finite subsets of $\mathbb{Z}$ and introduce the following Gibbsian specification.

Definition 6. Let $\beta>0$ be the inverse temperature. We call a Dyson specification the collection of probability kernels $\gamma^{D}=\left(\gamma_{\Lambda}^{D}\right)_{\Lambda \in \mathscr{L}}$ from $\mathcal{F}_{\Lambda^{c}}$ to $\Omega_{\Lambda}$ defined by

$$
\gamma_{\Lambda}^{D}(\sigma \mid \omega)=\frac{1}{Z_{\Lambda}^{\omega}} e^{-\beta H_{\Lambda}^{\omega}(\sigma)}
$$

where the normalization $Z_{\Lambda}^{\omega}$ is the usual partition function.

The specification $\gamma^{D}$ is monotonicity-preserving (or FKG): for all $\Lambda \in \mathscr{L}$ and any $f$ bounded increasing, so is $\gamma_{\Lambda}^{D} f$. The extremal (maximal and minimal) elements of this partial order " $\leq$ " already allow us to define the extremal elements of $\mathcal{G}\left(\gamma^{D}\right)$. Moreover, the weak limits

$$
\mu^{-}(\cdot):=\lim _{\Lambda} \gamma_{\Lambda}^{D}(\cdot \mid-) \text { and } \mu^{+}(\cdot):=\lim _{\Lambda} \gamma_{\Lambda}^{D}(\cdot \mid+)
$$

are well-defined, translation-invariant and extremal elements of $\mathcal{G}\left(\gamma^{D}\right)$.

For $1<\alpha \leq 2$, the Dyson model undergoes a phase transition at low temperature. There exists $\beta_{c}^{D}>0$ such that, for all $\beta>\beta_{c}^{D}$, we have $\mu^{-} \neq \mu^{+}$and moreover, for every $\beta>\beta_{c}^{D}$,

$$
\mathcal{G}\left(\gamma^{D}\right)=\left[\mu^{-}, \mu^{+}\right]
$$

See $[54,66,77,96]$ for the details. 
The Dobrushin boundary condition is the configuration $\omega \in \Omega$ defined by

$$
\omega_{i}= \begin{cases}+1, & \text { if } i \geq 0 \\ -1, & \text { if } i<0\end{cases}
$$

We denote the Gibbs measure with Dobrushin boundary condition by $\mu^{-+}$.

To get a candidate to represent the $g$-functions, i.e. the conditional probabilities w.r.t. the past, one needs to extend (3.37) to possibly infinite sets $\Pi$, because the complement of the past - our future - is infinite. Although we are far from the uniqueness regime, this has nevertheless been shown to be possible in our context following a general construction of [66], made for attractive and right-continuous ${ }^{3}$ specifications.

Definition 7. A Global Specification $\Gamma$ on $\mathbb{Z}$ is a family of probability kernels $\Gamma=\left(\Gamma_{\Pi}\right)_{\Pi \subset \mathbb{Z}}$ on $(\Omega, \mathcal{F})$ with $\Gamma_{\Pi}: \mathcal{F}_{\Pi^{c}} \times \Omega_{\Pi} \rightarrow \mathbb{R}$ such that, for any subset $\Pi$ of $\mathbb{Z}$,

1. $\Gamma_{\Pi}(B \mid \omega)=\mathbf{1}_{B}(\omega)$ for all $\omega \in \Omega$ when $B \in \mathcal{F}_{\Pi^{c}}$.

2. For all $\Pi_{1} \subset \Pi_{2} \subset \mathbb{Z}$, we have $\Gamma_{\Pi_{2}} \Gamma_{\Pi_{1}}=\Gamma_{\Pi_{2}}$.

We write $\mu \in \mathcal{G}(\Gamma)$ if for all $A \in \mathcal{F}$ and any $\Pi \subset \mathbb{Z}$,

$$
\mu\left[A \mid \mathcal{F}_{\Pi^{c}}\right](\omega)=\Gamma_{\Pi}(A \mid \omega), \mu-\text { a.e. } \omega .
$$

Following the construction of Fernández and Pfister's result [66] in the general monotonicitypreserving case, van Enter and Le Ny [59] showed the following result.

Theorem 24. Consider the Dyson model on $\mathbb{Z}$ at inverse temperature $\beta>0$, i.e. the specification $\gamma^{D}$ given by (3.37) and its extremal Gibbs measure $\mu^{+}$defined by (3.38). A global specification $\Gamma^{+}$ such that $\mu^{+} \in \mathcal{G}\left(\Gamma^{+}\right)$can be given as follows:

- For $\Pi=\Lambda$ finite, for all $\omega \in \Omega$, set $\Gamma_{\Lambda}^{+}(d \sigma \mid \omega):=\gamma_{\Lambda}^{D}(d \sigma \mid \omega)$.

- For $\Pi$ infinite, for all $\omega \in \Omega$, set $\Gamma_{\Pi}^{+}(d \sigma \mid \omega):=\mu_{\Pi}^{\omega,+} \otimes \delta_{\omega_{\Pi c}}(d \sigma)$ where $\mu_{\Pi}^{\omega,+}$ is the constrained measure on $\left(\Omega_{\Pi}, \mathcal{F}_{\Pi}\right)$ defined as the (well-defined) weak limit

$$
\mu_{\Pi}^{\omega,+}\left(\mathrm{d} \sigma_{\Pi}\right):=\lim _{\Delta \uparrow \Pi} \gamma_{\Delta}^{D}\left(\mathrm{~d} \sigma \mid \omega_{\Pi^{c}+\Pi}\right)
$$

A similar construction yields a global specification $\Gamma^{-}$so that $\mu^{-} \in \mathcal{G}\left(\Gamma^{-}\right)$.

These constructions allow us to consider, for given pasts, the expression of the $g$-functions as the magnetisations of Dyson models under various conditionings, see equation (3.44) below, and studying continuity will reduce to studying possible phase transition under constraints combined with the study of the stability of interfaces.

Starting from $\mu^{+}$, we introduce $g^{+}$to be the candidate to be the $g$-function representing (a version of) the single-site conditional probabilities (Theorem 16, item 2), as a function of the past. Just as in $[59,66]$, we introduce thus for any "past" configuration $\omega \in \Omega$ :

$$
g^{+}(\omega):=\mu^{+}\left(\left[\omega_{0}\right] \mid \mathcal{F}_{<0}\right)(\omega) .
$$

Using the expression of Theorem 24 in terms of global specifications and constrained measures with $\Pi=\mathbb{Z}_{+}=\{0,1,2,3, \ldots\}$, one gets, for $\mu^{+}$-a.s. $\omega$,

$$
g^{+}\left(\omega_{-\infty}^{-1} \sigma_{0}\right)=\Gamma_{\mathbb{Z}_{+}}^{+}\left(\left[\sigma_{0}\right] \mid \omega\right)=\mu_{\mathbb{Z}_{+}}^{\omega,+} \otimes \delta_{\omega_{\mathbb{Z}_{+}^{c}}}\left(\left[\sigma_{0}\right]\right), \quad \text { for every } \sigma_{0} \in\{-1,1\}
$$

\footnotetext{
${ }^{3}$ Right- or left-continuity corresponds to "continuity in the direction + or -", see e.g. [130].
} 
where $\mu_{\mathbb{Z}_{+}}^{\omega,+}$ is the constrained measure on $\left(\Omega_{\mathbb{Z}_{+}}, \mathcal{F}_{\mathbb{Z}_{+}}\right)$as the (well-defined) weak limit

$$
\mu_{\mathbb{Z}_{+}}^{\omega,+}\left(\mathrm{d} \sigma_{\mathbb{Z}_{+}}\right)=\lim _{\Delta \uparrow \mathbb{Z}_{+}} \gamma_{\Delta}^{D}\left(\mathrm{~d} \sigma \mid \omega_{\mathbb{Z}_{+}^{c}}+\mathbb{Z}_{+}\right) .
$$

Previous works and specific properties ${ }^{4}$ insure $\mu^{+}$is then indeed "specified" by $g^{+}$, in the sense that it is invariant by its left action: $\mu^{+} g^{+}=\mu^{+}$.

An essentially non-continuous (= non-regular) $g$-function gives rise to a measure which is NOT a $g$-measure. To be a "proper" $g$-function of the past, we would need that in addition to consistency, the function $g^{+}$is essentially continuous.

Discontinuity of any candidate $g^{+}$to represent a $g$-function for $\mu^{+}$- i.e. discontinuity of any possible version of a suitable chosen conditional probability - will be a consequence of the next lemma, proved using an entropic repulsion phenomenon, which we obtain as a fairly direct corollary of the interface localisation result of [41]. To use these results of Cassandro et al., we will require the same technical lower bound $\alpha \in\left(\alpha_{+}, 2\right)$, where $\alpha_{+} \in(1,2)$ is given by

$$
\alpha_{+}:=3-\frac{\log 3}{\log 2} .
$$

In the following lemma, $\mu_{\mathbb{Z}_{+}}^{\omega,+}(f)$ denotes expectation of $f$ under the constrained measure $\mu_{\mathbb{Z}_{+}}^{\omega,+}$.

Lemma 1. Consider the alternating configuration $\omega_{\mathrm{alt}}=\left(\left(\omega_{\mathrm{alt}}\right)_{i}\right)_{i \in \mathbb{Z}}$ defined by $\left(\omega_{\mathrm{alt}}\right)_{i}=(-1)^{i}$, and take a Dyson model with polynomial decay $\alpha_{+}<\alpha<2$ at sufficiently low temperature. Then, there exist $L_{0} \geq 1$ and $\delta>0$ such that for any $L>L_{0}$ there is an $N>L$, with $L N^{1-\alpha}=o(1)$, such that for every two configurations $\omega^{+} \in \mathcal{N}_{N, L}^{+, \text {left }}\left(\omega_{\text {alt }}\right)$ and $\omega^{-} \in \mathcal{N}_{N, L}^{-, \text {left }}\left(\omega_{\text {alt }}\right)$,

$$
\left|\mu_{\mathbb{Z}_{+}}^{\omega^{+},+}\left(\sigma_{0}\right)-\mu_{\mathbb{Z}_{+}}^{\omega^{-},+}\left(\sigma_{0}\right)\right|>\delta
$$

As a corollary, we obtain our main result.

Theorem 25. For $\mu$ being either the plus or the minus phase of a Dyson model with exponent $\alpha_{+}<\alpha<2$ at sufficiently low temperature, the one-sided conditional probability $\mu\left(\left[\omega_{0}\right] \mid \mathcal{F}_{<0}\right)(\cdot)$ is essentially discontinuous at $\omega_{\text {alt }}$. Therefore, none of the Gibbs measures $\mu$ for the Dyson model in this phase transition region ${ }^{5}$ is a g-measure.

Similarly to e.g. [59], which exhibited (two-sided) discontinuity points by considering an alternating configuration $\omega_{\text {alt }}$, we will prove that for $L$ large and $N$ large compared to $L$, the putative $g$-function $g^{+}$can take significantly different values on sub-neighborhoods $\mathcal{N}_{N, L}^{ \pm \text {,left }}\left(\omega_{\text {alt }}\right) \subset \mathcal{N}_{L}\left(\omega_{\text {alt }}\right)$. To prove the essential discontinuity and in some sense "some" wetting beyond the origin through the alternating region, we first use the interface result of [41] (see also [40]) to state and prove in Section 3.3 a wetting result that we relate to entropic repulsion (see e.g. [137], or [85] for similar terminology in the setting of random polymers).

\subsubsection{Interfaces in Dyson models}

We will thus derive our entropic repulsion argument from the interface result of [41]. We start by describing and summarizing the latter and in particular briefly recall the contour construction based on triangles, that was first described in [39] to formalize the contour argument of [77]. Then we describe the Peierls estimate they obtain in this one-dimensional long-range context. In addition, this triangle construction also allows an unambiguous notion of interface in the phase transition region, as we describe now.

Let $L \geq 1$, and consider $\Lambda=\Lambda_{L}=[-L, L]$. Define the dual lattice $\Lambda^{*}=\Lambda+\frac{1}{2}$ as the set $\Lambda$ shifted by $1 / 2$. Given a configuration $\omega \in\{-1,+1\}^{\Lambda}$, let us define configurations of triangles. A

\footnotetext{
${ }^{4}$ Attractivity and right-continuity, see previous footnote and also [59, 66].

${ }^{5}$ Note that we again impose the technical restriction $\alpha_{+}<\alpha<2$ on the decay parameter.
} 
spin-flip point is a site $i$ in $\Lambda^{*}$ such that $\omega_{i-\frac{1}{2}} \neq \omega_{i+\frac{1}{2}}$. For each spin-flip point $i$, let us consider the interval $\left[i-\frac{1}{100}, i+\frac{1}{100}\right] \subset \mathbb{R}$ and choose a real number $r_{i}$ in this interval such that, for every four distinct points $r_{i_{1}}, r_{i_{2}}, r_{i_{3}}, r_{i_{4}}$, we have $\left|r_{i_{1}}-r_{i_{2}}\right| \neq\left|r_{i_{3}}-r_{i_{4}}\right|$. The $r_{i}$ 's will be the bases of the triangles, and the last condition is asked to avoid ambiguity in the construction of the triangle.

For each spin-flip point $i$, we start growing a " $\vee$-line" at $r_{i}$ where this $\vee$-line is embedded in $\mathbb{R}^{2}$ with angles $\pi / 4$ and $3 \pi / 4$. If at some time two $\vee$-lines starting from different spin-flip points touch, the other two lines starting from those two spin-flip points stop growing, and are removed without forming a triangle. Then we repeat this procedure. This process can also be seen in the following way: for each $r_{i}$, draw a straight vertical line passing through it. Take the smallest distance between these lines, call the correponding $r_{i}$ and $r_{j}$ the spin-flip points of these lines, and draw a isosceles triangle with base angle $\pi / 4$. Then, remove the lines associated to $r_{i}$ and $r_{j}$. Re-start.

Note that, for homogeneous boundary conditions, since the number of spin-flip points is even, every $r_{i}$ is a vertex of some triangle. On the other hand, if we consider the Dobrushin boundary condition, then the number of spin-flip points is odd, and so there exists a unique spin-flip point which is not the vertex of any triangle. This point is called the "interface point".

The first notion of interface point in this long-range one-dimensional context appeared in [107] in the terms of a "thick interface", and afterwards [41] defined the interface point according to the construction above.

Let

$$
\begin{aligned}
T_{L} & :=\left\{\frac{k}{2 L}: k \in[-2 L-1,2 L+1]\right\} \\
& =\left\{-1-\frac{1}{2 L},-1+\frac{1}{2 L}, \ldots,-\frac{1}{2 L}, \frac{1}{2 L}, \ldots, 1+\frac{1}{2 L}\right\},
\end{aligned}
$$

and consider the Dobrushin boundary condition with all spins to the left of $\Lambda$ fixed to be minus and all spins to the right of $\Lambda$ fixed to be plus. Given a configuration $\omega$ in $\Lambda$, let $I^{*} \equiv I^{*}(\omega) \in \Lambda^{*}$ be the interface point of the configuration $\omega$, and given $\theta \in T_{L}$, denote by

$$
\Omega_{\Lambda, \theta}:=\left\{\omega \in \Omega_{\Lambda}^{-+}: I^{*}=\theta L\right\}
$$

the set of spin configurations in $\Lambda$ for which the interface point is situated in $\theta L$. Note that this forms a partition of $\Omega$ (if $\theta \neq \theta^{\prime}$, then $\Omega_{\Lambda, \theta} \cap \Omega_{\Lambda, \theta^{\prime}}=\emptyset$ ). We also consider the definitions (3.48) and (3.49) for translated intervals $\Delta=[a-L, a+L]$ where $a \in \mathbb{Z}$. In that case,

$$
\begin{aligned}
T_{\Delta} & :=a+T_{L}=\left\{a+\theta: \theta \in T_{L}\right\} . \\
\Omega_{\Delta, \theta} & :=\left\{\omega \in \Omega_{\Delta}^{-+}: I^{*}=\theta L\right\}, \text { with } \theta \in T_{\Delta} .
\end{aligned}
$$

We use it to define for each $\theta \in T_{L}$ the probability to have an interface in $\theta L$ by

$$
\mu_{\Lambda}^{-+}\left(I^{*}=\theta L\right):=\frac{Z_{\theta, \Lambda}^{-+}}{Z_{\Lambda}^{-+}}
$$

where the partition functions

$$
Z_{\theta, \Lambda}^{-+}=\sum_{\omega \in \Omega_{\Lambda, \theta}} e^{-\beta H_{\Lambda}^{-+}(\omega)} \text { and } Z_{\Lambda}^{-+}=\sum_{\theta \in T_{L}} Z_{\theta, \Lambda}^{-+}
$$

are defined via the Hamiltonian $H_{\Lambda}^{-+}$in volume $\Lambda$ with Dobrushin boundary conditions.

For $i \in \Lambda$, the conditional expectation of $\omega_{i}$, given $I^{*}=\theta L$, is

$$
\mu_{\theta, \Lambda}^{-+}\left(\omega_{i}\right):=\mu_{\Lambda}^{-+}\left(\omega_{i} \mid I^{*}=\theta L\right)=\frac{1}{Z_{\theta, \Lambda}^{-+}} \sum_{\omega \in \Omega_{\Lambda, \theta}} \omega_{i} e^{-\beta H_{\Lambda}^{-+}(\omega)} .
$$


Moreover, the expectation of $\omega_{i}$ in terms of in terms of $\mu_{\theta, \Lambda}^{-+}\left[\omega_{i}\right]$ is

$$
\mu_{\Lambda_{L}}^{-+}\left(\omega_{i}\right)=\sum_{\theta \in T_{L}} \mu_{\theta, \Lambda_{L}}^{-+}\left(\omega_{i}\right) \mu_{\Lambda_{L}^{+}}^{-+}\left(I^{*}=\theta L\right)
$$

These constructions of triangles and associated contours are used in [41] to get cluster expansions of partition functions that yield first the following proposition, which will be an essential tool for us. Let $Z_{\Lambda}^{-}$be the partition function on $\Lambda$ with minus boundary condition, and let

$$
\zeta(\alpha)=\sum_{k=1}^{\infty} \frac{1}{k^{\alpha}}
$$

be the Riemann zeta function. For two real-valued numbers $x$ and $y$ with $y \geq 0$, we write $x= \pm y$ if $|x| \leq y$.

Proposition 2. For all $\alpha \in\left(\alpha_{+}, 2\right)$, there exists $\beta_{0} \equiv \beta_{0}(\alpha)>0$ such that for all $\beta>\beta_{0}$ and $\theta \in T_{L}$, the following occurs:

$$
\begin{aligned}
& \log Z_{\theta, \Lambda}^{-+}-\log Z_{\Lambda}^{-} \\
& =-\beta c_{L}(\alpha) L^{2-\alpha}+e^{-2 \beta(\zeta(\alpha)+J)} \frac{L^{2-\alpha}}{(2-\alpha)(\alpha-1)} f_{\alpha}(\theta)\left(1 \pm e^{-c_{1}(\alpha) \beta}\right)(1+o(1)),
\end{aligned}
$$

where $f_{\alpha}(\theta)=(1+\theta)^{2-\alpha}+(1-\theta)^{2-\alpha}, c_{L}$ and $c_{1}$ are two positive constants depending on $\alpha$, once we require that the nearest-neighbor interaction $J=J(1) \gg 1$, and o(1) converges to zero when $L \rightarrow \infty$.

The restriction of $\alpha>\alpha_{+}$appears since in [39] the proof of the phase transition of the Dyson model by a contour argument needs it $^{6}$, while the contours introduced are based on the triangles defined above.

From Proposition 2 and the observation that, at finite volume $\Lambda$, for any $i \in \Lambda$,

$$
\mu_{\theta, \Lambda}^{-+}\left(\omega_{i}\right)=\left.\frac{\mathrm{d}}{\mathrm{d} g}\left(\log Z_{\theta, \Lambda}^{g, i}\right)\right|_{g=0}
$$

where for any $g \in \mathbb{R}$,

$$
Z_{\theta, \Lambda}^{g, i}=\sum_{\sigma_{\Lambda} \in \Omega_{\Lambda, \theta}} e^{-\beta H_{\Lambda}^{-+}\left(\sigma_{\Lambda}\right)+g \sigma_{i}},
$$

Cassandro et al. also obtained in [41] the following estimate for important conditional magnetisations, which will provide our first step towards wetting and entropic repulsion in the next section.

Proposition 3. For all $\alpha \in\left(\alpha_{+}, 2\right]$, there exists $\beta_{0} \equiv \beta_{0}(\alpha)$ such that for all $\beta>\beta_{0}$, the spins left and right of the interface point are minus, respectively plus; for other sites we can compute the expected local magnetisation. In other words,

$$
\mu_{\theta, \Lambda}^{-+}\left(\omega_{i}\right)= \begin{cases}1, & \text { if } i=\theta L+\frac{1}{2} \\ -1, & \text { if } i=\theta L-\frac{1}{2}\end{cases}
$$

and

$$
\begin{aligned}
\mu_{\theta, \Lambda}^{-+}\left(\omega_{i}\right)= & {\left[1-2 e^{-2 \beta(\zeta(\alpha)+J)} e^{\frac{2 \beta}{\alpha-1} \frac{1}{|i-\theta L|^{\alpha-1}}}\left(1+O\left(e^{-c_{1}(\alpha) \beta}\right)\right)(1+o(1))\right] } \\
& \times\left[\mathbb{1}_{i>\theta L+\frac{1}{2}}-\mathbb{1}_{i<\theta L-\frac{1}{2}}\right] .
\end{aligned}
$$

\footnotetext{
${ }^{6}$ Although for the existence of a transition the validity can be extended to the whole range of phase-transition decays by FKG arguments. This does not work for inhomogeneous situations such as disordered systems [42] or interface fluctuations [41].
} 
Moreover, Cassandro et al. [41] also showed the following estimate for the magnetisation with plus boundary condition.

Theorem 26. For all $\alpha \in\left(\alpha_{+}, 2\right)$, there exists a $\beta_{0} \equiv \beta_{0}(\alpha)$ and a strictly positive constant $c_{1}$ such that for all $\beta \geq \beta_{0}$, uniformly with respect to $\Lambda \subset \mathbb{Z}$, for all $i \in \Lambda$ we have

$$
\left\langle\omega_{i}\right\rangle_{\Lambda}^{+}=1-\left[2 e^{-2 \beta(\zeta(\alpha)+J)}\left(1 \pm e^{-c_{1}(\alpha) \beta}\right)(1+o(1))\right] .
$$

Thus, after the thermodynamical limit, the magnetisation satisfies the following inequality at low temperature,

$$
1-\left[2 e^{-2 \beta(\zeta(\alpha)+J)}\left(1+e^{-c_{1}(\alpha) \beta}\right)\right] \leq\left\langle\omega_{i}\right\rangle^{+} \leq 1-\left[2 e^{-2 \beta(\zeta(\alpha)+J)}\left(1-e^{-c_{1}(\alpha) \beta}\right)\right] .
$$

From Proposition 2 and Proposition 3, the main theorem of Cassandro et al. [41] claims that the interface point is located in the middle of the interval of $\Lambda$, up to a Gaussian correction which grows sublinearly in $L$. This means that the correction describes mesoscopic fluctuations. In particular, this implies that macroscopic fluctuations are extremely improbable.

Theorem 27. For all $\alpha \in\left(\alpha_{+}, 2\right)$, there exists $\beta_{0} \equiv \beta_{0}(\alpha)$ such that for all $\beta>\beta_{0}$, if we denote $\frac{I^{*}}{L^{\alpha / 2}}=\mathcal{I}_{\alpha}^{*}$ then for all $s \in \mathbb{R}$,

$$
\lim _{L \rightarrow \infty} \mu_{\Lambda}^{-+}\left(\mathcal{I}_{\alpha}^{*} \leq s\right)=\int_{-\infty}^{s} \gamma_{\sigma(\beta, \alpha)}(z) \mathrm{d} z
$$

where $\gamma_{\sigma}(t)$ is the Gaussian density with mean zero and variance $\sigma^{2}=\sigma^{2}(\beta, \alpha)$,

$$
\gamma_{\sigma}(t)=\frac{1}{\sigma \sqrt{2 \pi}} e^{-\frac{1}{2} \cdot \frac{t^{2}}{\sigma^{2}}}
$$

and, for $\beta \geq \beta_{0}$, the variance $\sigma^{2}(\beta, \alpha)$ can be expressed as

$$
\sigma^{2}(\beta, \alpha)=\frac{1}{2} e^{2 \beta(J+\zeta(\alpha))}\left(1 \pm e^{-c_{1}(\alpha) \beta}\right)^{-1}
$$

for some constant $c_{1}(\alpha)$. Moreover for all $s \in \mathbb{R}$,

$$
\begin{aligned}
& m(s, \beta)=\lim _{L \rightarrow \infty} \mu_{\Lambda}^{-+}\left(\sigma_{\left[s L^{\alpha / 2}\right]}\right) \\
& =\left[1-2 e^{-2 \beta(\zeta(\alpha)+J)}\left(1 \pm e^{-c_{1}(\alpha) \beta}\right)\right]\left(\int_{-\infty}^{s} \gamma_{\sigma(\beta, \alpha)}(z) \mathrm{d} z-\int_{s}^{\infty} \gamma_{\sigma(\beta, \alpha)}(z) \mathrm{d} z\right),
\end{aligned}
$$

where $\left[s L^{\alpha / 2}\right]$ is the integer part of $s L^{\alpha / 2}$.

By Theorem 27 we can compute the following limit,

$$
\begin{aligned}
\lim _{L \rightarrow \infty} \mu_{\Lambda}^{-+}\left(\left|\mathcal{I}_{\alpha}^{*}\right| \leq s\right) & =\lim _{L \rightarrow \infty} \mu_{\Lambda}^{-+}\left(-s \leq \mathcal{I}_{\alpha}^{*} \leq s\right) \\
& =\lim _{L \rightarrow \infty} \mu_{\Lambda}^{-+}\left(\mathcal{I}_{\alpha}^{*} \leq s\right)-\mu_{\Lambda}^{-+}\left(\mathcal{I}_{\alpha}^{*} \leq-s\right) \\
& =\int_{-s}^{s} \gamma_{\sigma(\beta, \alpha)}(z) \mathrm{d} z
\end{aligned}
$$

\subsection{Entropic repulsion - Wetting transition}

For a fixed $N>1$, we will consider the plus phase $\mu^{+}$, conditioned on the event ${ }_{-}{ }_{N,-1}$ of there being an interval $[-N,-1]$ of minus spins. We claim that there are two intervals of length of order $L$, namely $\left[-N-\frac{\left(1-s L^{\frac{\alpha}{2}-1}\right)}{2} L,-N-1\right]$, and $\left[0, \frac{\left(1-s L^{\frac{\alpha}{2}-1}\right)}{2} L\right]$, left and right of the fixed interval, such 
that for $N \gg L$ both large enough, satisfying $L N^{1-\alpha}=o(1)$, the magnetisation of the spins in $\Xi$ conditioned on the event $-{ }_{-N,-1}$ is negative, whenever $\Xi$ is in one of those intervals. These intervals play the role of a "completely wet region" in a wetting transition" (See Figure 3.2). In other words,

Proposition 4. Let $\alpha \in\left(\alpha_{+}, 2\right)$ and $\beta_{0} \equiv \beta_{0}(\alpha)$ from Theorem 27. Then, there exists $\beta_{1}>\beta_{0}$ such that, for any $\beta>\beta_{1}$, there exist $s=s(\beta, \alpha), \lambda=\lambda(\beta, \alpha, s)>0$ and $L_{0} \equiv L_{0}(\alpha, \beta) \geq 1$ such that, for any $L>L_{0}$, there exists $N_{0}(L)>L$ such that, for any $N \geq N_{0}(L)$,

$$
\mu^{+}\left(\omega_{i} \mid--N,-1\right) \leq-\lambda m
$$

for every $i \in\left[-N-\frac{\left(1-s L^{\frac{\alpha}{2}-1}\right)}{2} L,-N-1\right] \cup\left[0, \frac{\left(1-s L^{\frac{\alpha}{2}-1}\right)}{2} L\right]$, where $m=\left\langle\omega_{0}\right\rangle^{+}>0$.

Proof. Fix $\alpha \in\left(\alpha_{+}, 2\right)$ and $\beta_{0} \equiv \beta_{0}(\alpha)$ from Proposition 2. We will first prove the statement for $i \in\left[0, \frac{\left(1-s L^{\frac{\alpha}{2}-1}\right)}{2} L\right]$.

The main idea of our proof is to choose $N$ large enough for the total influence of all spins left of the interval to be bounded by a (small) constant, so that one can neglect boundary effects beyond $-N$ as in [34]. Then inside the interval of length $L$, the interface separating the plus and minus phases is with large probability within the same window as with the Dobrushin boundary conditions. If afterwards we move the plus-boundary to the right, the location of the interface can also move only to the right, that is away from the frozen interface (by an FKG argument).

To make this precise we proceed as follows. Since $\mu^{+}$is translational invariant, it is enough to show

$$
\mu^{+}\left(\omega_{i} \mid-{ }_{-N,-L-1}\right)<-\lambda m,
$$

for every $i \in \Xi_{L}:=\left[-L, \frac{-L-s L^{\frac{\alpha}{2}}}{2}\right]$ and $N>L+1$ large enough. From Theorem 27, if we would consider the interval $\Lambda=[-L, L]$ with Dobrushin boundary condition, the interface point will with overwhelming probability lie about halfway, with fluctuations which are "mesoscopic", that is, there exists $\beta_{0}>0$ such that, for every $\beta>\beta_{0}$ and $s \geq 0$,

$$
\lim _{L \rightarrow \infty} \mu_{\Lambda}^{-+}\left(\left|I^{*}\right| \leq s L^{\alpha / 2}\right)=\int_{-s}^{s} \gamma_{\sigma(\beta, \alpha)}(z) \mathrm{d} z .
$$

Thus, for a fixed $\varepsilon>0$, there exists $L_{\varepsilon} \geq 1$ such that, for every $L \geq L_{\varepsilon}$,

$$
\left|\mu_{\Lambda}^{-+}\left(\left|I^{*}\right| \leq s L^{\alpha / 2}\right)-\int_{-s}^{s} \gamma_{\sigma(\beta, \alpha)}(z) \mathrm{d} z\right|<\varepsilon
$$

Let us take $i \in \Xi_{L}$. Note that, for every $\theta \in T_{L}$ with $-s L^{\alpha / 2} \leq \theta L \leq s L^{\alpha / 2}$, we have

$$
\begin{aligned}
& |i-\theta L|^{\alpha-1} \geq\left(\frac{L-s L^{\alpha / 2}}{2}\right)^{\alpha-1} \cdot \\
& -L
\end{aligned}
$$

Figure 3.1: Mesoscopic interval and the wet region.

By Proposition 3 and Inequality (3.61), for every $\beta \geq \beta_{0}$,

$$
\mu_{\theta, \Lambda}^{-+}\left(\omega_{i}\right)=-1+2 e^{-2 \beta(\zeta(\alpha)+J)} e^{\frac{2 \beta}{\alpha-1} \frac{1}{|i-\theta L|^{\alpha-1}}}\left[1+O\left(e^{-c_{1} \beta}\right)\right][1+o(1)]
$$

\footnotetext{
${ }^{7}$ Note that this wetting is a positive-temperature effect. Indeed, at zero temperature the interface with Dobrushin boundary conditions is homogeneously distributed, and a frozen interval of minuses, inserted in a plus configuration, will have only pluses to the left and to the right.
} 


$$
\begin{aligned}
& \leq-1+2 e^{-2 \beta(\zeta(\alpha)+J)} e^{\frac{2 \beta}{\alpha-1}\left(\frac{2}{L-s L^{\alpha / 2}}\right)^{\alpha-1}}\left[1+O\left(e^{-c_{1} \beta}\right)\right][1+o(1)] \\
& \leq-1+(1-m) e^{\frac{2 \beta}{\alpha-1}\left(\frac{2}{L-s L^{\alpha / 2}}\right)^{\alpha-1}}\left[\frac{1+O\left(e^{-c_{1} \beta}\right)}{1-e^{-c_{1} \beta}}\right][1+o(1)]
\end{aligned}
$$

For each $\delta>0$, there exist $\beta_{\delta}>\beta_{0}$ such that, for every $\beta>\beta_{\delta}$, there exists $L_{\beta} \geq 1$ such that, for every $L \geq L_{\beta}$,

$$
e^{\frac{2 \beta}{\alpha-1}\left(\frac{2}{L-s L^{\alpha / 2}}\right)^{\alpha-1}}\left[\frac{1+O\left(e^{-c_{1} \beta}\right)}{1-e^{-c_{1} \beta}}\right][1+o(1)]<1+\delta .
$$

Since $m(\beta) \rightarrow 1$ as $\beta \rightarrow \infty$, there exists $\beta_{1}>\beta_{\delta}$ such that, for every $\beta \geq \beta_{1}$,

$$
\mu_{\theta, \Lambda}^{-+}\left(\omega_{i}\right) \leq-1+(1-m)(1+\delta)<0 .
$$

Define

$$
\Theta_{s, L}=\left\{\theta \in T_{L}:|\theta| \leq s L^{\alpha / 2-1}\right\} .
$$

By (3.53), (3.70) and (3.73), for every $i \in \Xi_{L}$, with $\beta \geq \beta_{1}$ and $L \geq \max \left\{L_{\varepsilon}, L_{\beta}\right\}$,

$$
\begin{aligned}
\mu_{\Lambda}^{-+}\left(\omega_{i}\right) & =\sum_{\theta \in \Theta_{s, L}} \mu_{\theta, \Lambda}^{-+}\left(\omega_{i}\right) \mu_{\Lambda}^{-+}\left(I^{*}=\theta L\right)+\sum_{\theta \notin \Theta_{s, L}} \underbrace{\mu_{\theta, \Lambda}^{-+}\left(\omega_{i}\right)}_{\leq 1} \mu_{\Lambda}^{-+}\left(I^{*}=\theta L\right) \\
& \leq(-1+(1-m)(1+\delta))\left(\int_{-s}^{s} \gamma_{\sigma(\beta, \alpha)}(z) \mathrm{d} z-\varepsilon\right)+\varepsilon+1-\int_{-s}^{s} \gamma_{\sigma(\beta, \alpha)}(z) \mathrm{d} z .
\end{aligned}
$$

Choose $s_{0} \geq 0$ such that, for $s \geq s_{0}$,

$$
1-\int_{-s}^{s} \gamma_{\sigma(\beta, \alpha)}(z) \mathrm{d} z<\varepsilon
$$

Writing

$$
-1+(1-m)(1+\delta)=-m+\delta(1-m)
$$

we have

$$
\begin{aligned}
\mu_{\Lambda}^{-+}\left(\omega_{i}\right) & \leq(-m+\delta(1-m))\left(\int_{-s}^{s} \gamma_{\sigma(\beta, \alpha)}(z) \mathrm{d} z-\varepsilon\right)+2 \varepsilon \\
& =-m \int_{-s}^{s} \gamma_{\sigma(\beta, \alpha)}(z) \mathrm{d} z+\varepsilon m+\delta(1-m)\left(\int_{-s}^{s} \gamma_{\sigma(\beta, \alpha)}(z) \mathrm{d} z-\varepsilon\right)+2 \varepsilon .
\end{aligned}
$$

Choose $\varepsilon>0$ and $\delta>0$ small enough (and so $\beta, L, s$ large enough) such that

$$
\varepsilon m+\delta(1-m)\left(\int_{-s}^{s} \gamma_{\sigma(\beta, \alpha)}(z) \mathrm{d} z-\varepsilon\right)+2 \varepsilon<\frac{m}{2} \int_{-s}^{s} \gamma_{\sigma(\beta, \alpha)}(z) \mathrm{d} z .
$$

Thus,

$$
\mu_{\Lambda}^{-+}\left(\omega_{i}\right) \leq-\frac{m}{2} \int_{-s}^{s} \gamma_{\sigma(\beta, \alpha)}(z) \mathrm{d} z<0 .
$$

For any $N>L+1$, if we lift the constraint that all spins are minus to the left of site $-N$, the total 
energy due to the boundary condition changing inside the interval $\Xi_{L}$ is bounded by

$$
\begin{aligned}
\left|\sum_{j<-N} \sum_{i=-L}^{L} \frac{1}{|i-j|^{\alpha}} \omega_{i} \omega_{j}\right| & \leq(2 L+1) \sum_{i>N} \frac{1}{i^{\alpha}} \\
& \leq 3 L \int_{N}^{+\infty} \frac{1}{x^{\alpha}} \mathrm{d} x \\
& \leq \frac{3}{\alpha-1} L N^{1-\alpha}
\end{aligned}
$$

Thus,

$$
\lim _{N \rightarrow \infty} \sum_{j<-N} \sum_{i=-L}^{L} \frac{1}{|i-j|^{\alpha}} \omega_{i} \omega_{j}=0
$$

for every configuration $\omega$. Let us denote by $\omega^{+}$be the plus configuration $\omega_{i}^{+}=+1$ for every $i \in \mathbb{Z}$, and by $\omega^{-}$be the minus configuration $\omega_{i}^{-}=-1$ for every $i \in \mathbb{Z}$. Consider $\tilde{\Lambda}=[-N, L]$. For a fixed $\omega$ such that $\omega_{i}=-1$ with $i \in[-N,-L-1]$,

$$
\begin{aligned}
H_{\tilde{\Lambda}}^{+}(\omega) & =-\sum_{i, j \in \tilde{\Lambda}} J_{i j} \omega_{i} \omega_{j}-\sum_{\substack{i \in \tilde{\Lambda} \\
j<-N}} J_{i j} \omega_{i} \omega_{j}^{+}-\sum_{\substack{i \in \tilde{\Lambda} \\
j>L}} J_{i j} \omega_{i} \omega_{j}^{+} \\
& =H_{\Lambda}^{-+}(\omega)-2 \sum_{\substack{i \in \Lambda \\
j<-N}} J_{i j} \omega_{i} \omega_{j}^{+}+C_{L, N},
\end{aligned}
$$

where $C_{L, N}$ does not depend on $\omega$. For $i \in \Xi_{L}$,

$$
\mu_{\tilde{\Lambda}}^{+}\left(\omega_{i} \mid--N,-L-1\right)=\frac{\mu_{\Lambda}^{-+}\left(\omega_{i} \exp \left(2 \beta \sum_{k \in \Lambda, j<-N} J_{k j} \omega_{k} \omega_{j}^{+}\right)\right)}{\mu_{\Lambda}^{-+}\left(\exp \left(2 \beta \sum_{k \in \Lambda, j<-N} J_{k j} \omega_{k} \omega_{j}^{+}\right)\right)} .
$$

Let us recall that $\mu_{\Lambda}^{-+}\left(\omega_{i}\right)<0$. By (3.79), there exists $N_{0}(L)>L+1$ such that, for every $N \geq N_{0}(L)$,

$$
\mu_{\tilde{\Lambda}}^{+}\left(\omega_{i} \mid--N,-L-1\right)<\frac{1}{2} \mu_{\Lambda}^{-+}\left(\omega_{i}\right)
$$

Thus, by (3.78),

$$
\mu_{\tilde{\Lambda}}^{+}\left(\omega_{i} \mid--N,-L-1\right)<-\frac{m}{4} \int_{-s}^{s} \gamma_{\sigma(\beta, \alpha)}(z) \mathrm{d} z=-\lambda m
$$

where

$$
\lambda=\frac{1}{4} \int_{-s}^{s} \gamma_{\sigma(\beta, \alpha)}(z) \mathrm{d} z .
$$

Due to the FKG property, for any $\Delta$ containing $\tilde{\Lambda}$, we have

$$
\mu_{\Delta}^{+}\left(\omega_{i} \mid-{ }_{-N,-L-1}\right) \leq \mu_{\tilde{\Lambda}}^{+}\left(\omega_{i} \mid-{ }_{-N,-L-1}\right),
$$

for all $i \in \Xi_{L}$. Therefore, for any site $i \in \Xi_{L}$, there exists $L_{0} \geq 1$ such that, for $L>L_{0}$ and $N \geq N_{0}(L)$,

$$
\mu^{+}\left(\omega_{i} \mid-{ }_{-N,-L-1}\right)<-\lambda m
$$

For the wetting of sites $i$ in the other interval $\left[-N-\frac{\left(1-s L^{\frac{\alpha}{2}-1}\right)}{2} L,-N-1\right]$, we consider the Gibbs measure with reverse Dobrushin boundary condition $\mu^{+-}$, i.e., $\omega_{i}=1$ if $i<0$, and $\omega_{i}=-1$ if $i \geq 0$, 
and apply the same argument as above. Thus, for $N$ large enough,

$$
\mu^{+}\left(\omega_{i} \mid-1, N\right)<-\lambda m
$$

for every $i \in\left[-\frac{\left(1-s L^{\frac{\alpha}{2}-1}\right)}{2} L, 0\right]$, where $-_{1, N}$ is the event of there being an interval $[1, N]$ of minus spins. Since the Dyson model is translational invariant, when we shift all sites by $-N$, we are done.

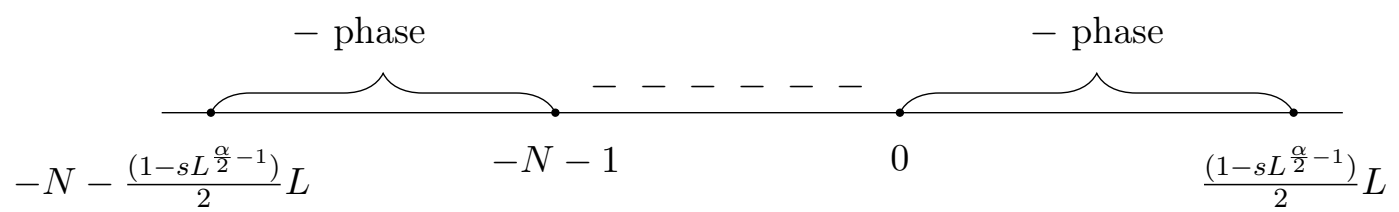

Figure 3.2: Wetting transition at low temperature.

\subsection{Lack of the $g$-measure property: proof}

In this section, we provide the proof of Theorem 25 .

The main idea is first to decouple the spins in a subinterval $\left[1, L_{1}\right]$ of the "wet" minus interval of length $o(L)$, such that $L_{1}$ is large, but sufficiently small compared to $L$. As the energy difference due to the decoupling is small compared to the free energy cost of moving the interface, the location of the interface as analyzed in [41] does not change, when viewed on scale $L$. If then, in the next step, the decoupled region is frozen in an alternating configuration and recoupled, this causes an extra finite-energy term -as compared to being decoupled-, which again will hardly influence the location of the interface (and thus the size of the wet region).

Let us first present a lemma.

Lemma 2. Let $\alpha \in(1,2)$ and $L_{1}>1$. Consider the observable $B$ in $\Omega$ given by

$$
B(\omega)=\sum_{\substack{i \in\left[-L_{1},-1\right] \\ j \notin\left[-L_{1},-1\right]}} \frac{(-1)^{i}}{|i-j|^{\alpha}} \omega_{j} .
$$

Then, there exists $c>0$ such that $\sup _{\omega}|B(\omega)|=\|B\| \leq c$, where $c$ does not depend on $L_{1}$.

Proof. From (3.87),

$$
\|B\| \leq \sum_{j \notin\left[-L_{1},-1\right]}\left|R_{j}\right|
$$

with

$$
R_{j}=\sum_{i \in\left[-L_{1},-1\right]} \frac{(-1)^{i}}{|i-j|^{\alpha}} .
$$

As $R_{j}$ is an alternating series with terms tending to zero monotonically in absolute value, its absolute value is no longer than that of its first term

$$
\left|R_{j}\right| \leq \frac{1}{|-1-j|^{\alpha}}
$$

Hence,

$$
\|B\| \leq \sum_{k \geq 1} \frac{1}{k^{\alpha}}:=c
$$


as we desired.

Bricmont, Lebowitz and Pfister [34] showed that, if two boundary conditions for general spin system on $\mathbb{Z}^{d}$ such that the difference in the energies of any spin configuration is uniformly bounded, then any finite-volume Gibbs states obtained with these boundary conditions have the same measure-zero sets. This implies that the decompositions of these Gibbs states into extremal Gibbs states are equivalent, i.e., mutually absolutely continuous. We will present a similar result.

Two Hamiltonian $\left(H_{\Lambda}\right)_{\Lambda \in \mathscr{L}}$ and $\left(H_{\Lambda}^{\prime}\right)_{\Lambda \in \mathscr{L}}$ have a finite energy difference if

$$
C=\sup _{\Lambda \in \mathscr{L}} \sup _{\sigma \in \Omega_{\Lambda}}\left|H_{\Lambda}(\sigma)-H_{\Lambda}^{\prime}(\sigma)\right|<\infty .
$$

A measure $\mu_{1}$ is absolutely continuous with respect to $\mu_{2}$ if $\mu_{2}(A)=0$ implies $\mu_{1}(A)=0$. We write $\mu_{1} \ll \mu_{2}$. Two measures $\mu_{1}$ and $\mu_{2}$ are equivalent if $\mu_{1} \ll \mu_{2}$ and $\mu_{2} \ll \mu_{1}$. The following statements are known:

(1) For any $\rho \in \mathcal{G}_{\beta}$, there exists an unique probability measure $\mu_{\rho}$ concentrated on the extremal set of Gibbs measures ex $\mathcal{G}_{\beta}$, such that

$$
\rho=\int \omega \mu_{\rho}(\mathrm{d} \omega)
$$

(2) Two Gibbs states are equivalent if and only if $\mu_{\rho}$ and $\mu_{\rho^{\prime}}$ are equivalent.

Proposition 5. Given two Hamiltonians $\left(H_{\Lambda}\right)_{\Lambda \in \mathscr{L}}$ and $\left(H_{\Lambda}^{\prime}\right)_{\Lambda \in \mathscr{L}}$ having a finite energy difference and a sequence $\Lambda_{n} \uparrow \mathbb{Z}^{d}$ such that

$$
\lim _{n \rightarrow \infty} \mu_{\Lambda_{n}, \beta}=\rho \quad \text { and } \quad \lim _{n \rightarrow \infty} \mu_{\Lambda_{n}, \beta}^{\prime}=\rho^{\prime} .
$$

then $\rho$ and $\rho^{\prime}$ are equivalent and so are $\mu_{\rho}$ and $\mu_{\rho^{\prime}}$.

Proof. Note that

$$
e^{-\beta C} \leq \frac{Z_{\Lambda, \beta}}{Z_{\Lambda, \beta}^{\prime}}=\frac{\sum_{\sigma \in \Omega_{\Lambda}} e^{-\beta H_{\Lambda}(\sigma)+\beta H_{\Lambda}^{\prime}(\sigma)} \cdot e^{-\beta H_{\Lambda}^{\prime}(\sigma)}}{\sum_{\sigma \in \Omega_{\Lambda}} e^{-\beta H_{\Lambda}^{\prime}(\sigma)}} \leq e^{\beta C} .
$$

Then, for every local function $\mathcal{F}_{\Lambda}$-measurable $f$, we have

$$
e^{-2 \beta C} \mu_{\Lambda, \beta}(f) \leq \mu_{\Lambda, \beta}^{\prime}(f) \leq e^{2 \beta C} \mu_{\Lambda, \beta}(f) .
$$

Letting $\Lambda=\Lambda_{n}$ and $n \rightarrow \infty$, we have

$$
e^{-2 \beta C} \rho(f) \leq \rho^{\prime}(f) \leq e^{2 \beta C} \rho(f),
$$

concluding that $\rho$ and $\rho^{\prime}$ are equivalent.

Proof of Lemma 1. For $L_{1} \geq 1$, consider

$$
A_{L_{1}}=\left\{\omega \in \Omega: \omega_{k}=\omega_{k}^{\text {alt }},-L_{1} \leq k<0\right\},
$$

Let $I=[-n, n]$ with $n$ large enough and $N>L_{1}$. Define the set

$$
\Psi=\left\{\{i, j\}: \text { Or }\{i, j\} \subset\left[-L_{1},-1\right] \text { either } i \in\left[-L_{1},-1\right], j \notin\left[-L_{1},-1\right]\right\} .
$$

Let $\tilde{H}_{I, L_{1}}^{\omega}$ be the Hamiltonian defined by

$$
\tilde{H}_{I, L_{1}}^{\omega}(\sigma)=H_{I}^{\omega}(\sigma)+\sum_{\substack{\{i, j\} \in \Psi \\ i, j \in I}} J_{i j} \sigma_{i} \sigma_{j}+\sum_{\substack{\{i, j\} \in \Psi \\ i \in I, j \notin I}} J_{i j} \sigma_{i} \omega_{j},
$$


i.e., we remove the interactions between $\left[-L_{1},-1\right]$ and its complement. Let $\tilde{\mu}_{I, L_{1}}^{\omega}$ be the probability measure defined by

$$
\tilde{\mu}_{I, L_{1}}^{\omega}(\sigma)=\frac{1}{\tilde{Z}_{I, L_{1}}^{\omega}} e^{-\beta \tilde{H}_{I, L_{1}}^{\omega}(\sigma)}
$$

where $\tilde{Z}_{I, L_{1}}^{\omega}$ is the partition function

$$
\tilde{Z}_{I, L_{1}}^{\omega}=\sum_{\sigma \in \Omega_{I}^{\omega}} e^{-\beta \tilde{H}_{I, L_{1}}^{\omega}(\sigma)}
$$

Note that there exists the thermodynamical limit of $\tilde{\mu}_{I, L_{1}}^{+}$,

$$
\lim _{n \rightarrow \infty} \tilde{\mu}_{I, L_{1}}^{+}:=\tilde{\mu}_{L_{1}}^{+}
$$

Computing the magnetisation given the set $A_{L_{1}}$, we have

$$
\mu_{I}^{+}\left(\omega_{0} \mid A_{L_{1}}\right)=\frac{\mu_{I}^{+}\left(\omega_{0} \cdot \mathbb{1}_{A_{L_{1}}}\right)}{\mu_{I}^{+}\left(A_{L_{1}}\right)}=\frac{\mu_{I}^{+}\left(\omega_{0} \cdot \exp (\beta B(\omega))\right)}{\mu_{I}^{+}(\exp (\beta B(\omega)))},
$$

where $B(\omega)$ is defined in (3.87). By Lemma 2,

$$
|B(\omega)|=\left|\sum_{\substack{i \in\left[-L_{1},-1\right] \\ j \notin\left[-L_{1},-1\right]}} J_{i j}(-1)^{i} \omega_{j}\right|<c,
$$

for every configuration $\omega$. Thus, by Proposition 5 , the measures $\mu^{+}\left(\cdot \mid A_{L_{1}}\right)$ and $\tilde{\mu}_{L_{1}}^{+}$are equivalent. Therefore, it is enough to show that the alternating configuration is an essentially discontinuous point for $\tilde{\mu}_{L_{1}}^{+}\left(\left[\omega_{0}\right] \mid \mathcal{F}_{<0}\right)(\cdot)$. In fact, we will show that every configuration is essentially discontinuous point for $\tilde{\mu}_{L_{1}}^{+}\left(\left[\omega_{0}\right] \mid \mathcal{F}_{<0}\right)(\cdot)$. Note that

$$
\left|H_{I}^{-+}(\omega)-\tilde{H}_{I, L_{1}}^{-+}(\omega)\right| \leq \sum_{\{i, j\} \in \Psi} J_{i j} \leq \kappa L_{1}^{2-\alpha},
$$

for some constant $\kappa>0$. Thus, for $\Lambda=[-L, L]$,

$$
\left|\log \tilde{Z}_{\theta, \Lambda, L_{1}}^{-+}-\log Z_{\theta, \Lambda}^{-+}\right| \leq \beta \kappa L_{1}^{2-\alpha},
$$

where $\tilde{Z}_{\theta, \Lambda, L_{1}}^{-+}$is defined as in (3.51). Let us choose $L_{1}=L^{\delta}$ with $\delta<1$, set $y=\theta L^{1-\frac{\alpha}{2}}$ and $\mathcal{Y}_{L}=T_{L} L^{1-\frac{\alpha}{2}}$. We have

$$
\mu_{\Lambda, L_{1}}^{-+}\left(\theta \leq s L^{-1+\frac{\alpha}{2}}\right)=\sum_{\theta \in T_{L}} \mathbb{1}_{\left\{\theta \leq s L^{-1+\frac{\alpha}{2}}\right\}} \frac{Z_{\theta, \Lambda, L_{1}}^{-+}}{Z_{\Lambda, L_{1}}^{-+}} \geq \sum_{\theta \in T_{L}} \mathbb{1}_{\left\{\theta \leq s L^{-1+\frac{\alpha}{2}}\right\}} \frac{Z_{\theta, \Lambda}^{-+}}{Z_{\Lambda}^{-+}} \exp \left\{-2 \beta \kappa L_{1}^{2-\alpha}\right\} .
$$

By Proposition 2,

$$
\begin{aligned}
& \mu_{\Lambda, L_{1}}^{-+}\left(\theta \leq s L^{-1+\frac{\alpha}{2}}\right) \geq \\
& \geq \frac{\sum_{y \in \mathcal{Y}_{L}} \mathbb{1}_{\{y \leq s\}} \exp \left\{\frac{1}{2} \sigma^{-2}(\beta, \alpha) \frac{L^{2-\alpha}}{(2-\alpha)(\alpha-1)} f_{\alpha}\left(y L^{-1+\frac{\alpha}{2}}\right)(1+o(1))-2 \beta \kappa L^{(2-\alpha) \delta}\right\}}{\sum_{z \in \mathcal{Y}_{L}} \exp \left\{\frac{1}{2} \sigma^{-2}(\beta, \alpha) \frac{L^{2-\alpha}}{(2-\alpha)(\alpha-1)} f_{\alpha}\left(z L^{-1+\frac{\alpha}{2}}\right)(1+o(1))\right\}}
\end{aligned}
$$




$$
=\frac{\sum_{y \in \mathcal{Y}_{L}} \mathbb{1}_{\{y \leq s\}} \exp \left\{\frac{1}{2} \sigma^{-2}(\beta, \alpha) L^{2-\alpha}\left(\frac{f_{\alpha}\left(y L^{-1+\frac{\alpha}{2}}\right)(1+o(1))}{(2-\alpha)(\alpha-1)}-o(1)\right)\right\}}{\sum_{z \in \mathcal{Y}_{L}} \exp \left\{\frac{1}{2} \sigma^{-2}(\beta, \alpha) \frac{L^{2-\alpha}}{(2-\alpha)(\alpha-1)} f_{\alpha}\left(z L^{-1+\frac{\alpha}{2}}\right)(1+o(1))\right\}} .
$$

Since, by [41],

$$
\begin{aligned}
& \lim _{L \rightarrow \infty} \frac{\sum_{y \in \mathcal{Y}_{L}} \mathbb{1}_{\{y \leq s\}} \exp \left\{\frac{1}{2} \sigma^{-2}(\beta, \alpha) \frac{L^{2-\alpha}}{(2-\alpha)(\alpha-1)} f_{\alpha}\left(y L^{-1+\frac{\alpha}{2}}\right)(1+o(1))\right\}}{\sum_{z \in \mathcal{Y}_{L}} \exp \left\{\frac{1}{2} \sigma^{-2}(\beta, \alpha) \frac{L^{2-\alpha}}{(2-\alpha)(\alpha-1)} f_{\alpha}\left(z L^{-1+\frac{\alpha}{2}}\right)(1+o(1))\right\}}= \\
& =\int_{-\infty}^{s} \frac{e^{-\frac{y^{2}}{2 \sigma^{2}(\beta, \alpha)}}}{\int_{-\infty}^{+\infty} e^{-\frac{z^{2}}{2 \sigma^{2}(\beta, \alpha)}} \mathrm{d} z} \mathrm{~d} y \\
& =\int_{-\infty}^{s} \gamma_{\sigma(\beta, \alpha)}(z) \mathrm{d} z .
\end{aligned}
$$

We showed that with large probability the interface still moves mesoscopically after we remove the bonds in $\Psi$ when $L_{1}$ increases sufficiently slower then $L$. Thus, we can apply Proposition 4 for the measure $\tilde{\mu}_{L_{1}}^{+}$. For $\beta$ large enough, there exists $\lambda=\lambda(\beta, \alpha)>0$ and $L_{0} \equiv L_{0}(\alpha, \beta) \geq 1$ such that, for any $L>L_{0}$, there exists $N_{0}(L)>L$ such that, for any $N \geq N_{0}(L)$,

$$
\tilde{\mu}_{L_{1}}^{+}\left(\omega_{i} \mid-{ }_{-N,-L_{1}}\right) \leq-\lambda m
$$

for every $i \in\left[0, \frac{\left(1-s L^{\frac{\alpha}{2}-1}\right)}{2} L\right]$. In particular, $\tilde{\mu}_{L_{1}}^{+}\left(\omega_{0} \mid--N,-L_{1}\right) \leq-\lambda m$.

Note that, if we consider the frozen interval of plus on $\left[-N,-L_{1}\right]$, we have

$$
\tilde{\mu}_{L_{1}}^{+}\left(\omega_{0} \mid+-N,-L_{1}\right) \geq \lambda m
$$

for $N$ sufficiently large. Thus,

$$
\tilde{\mu}_{L_{1}}^{+}\left(\omega_{i} \mid+-N,-L_{1}\right)-\tilde{\mu}_{L_{1}}^{+}\left(\omega_{i} \mid-{ }_{-N,-L_{1}}\right) \geq 2 \lambda m,
$$

concluding the proof.

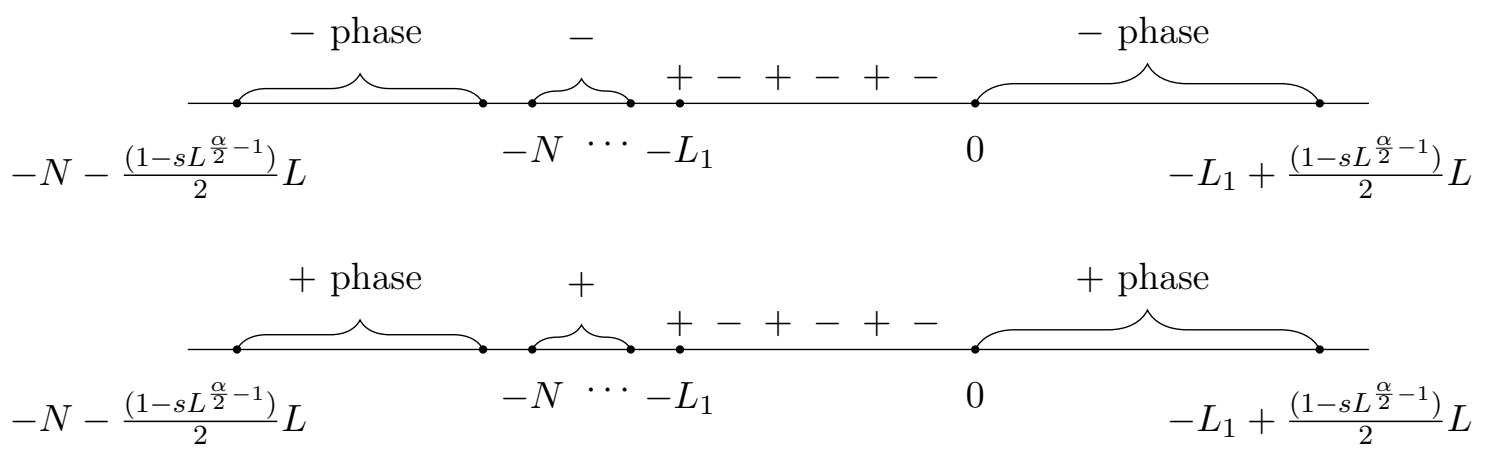

Figure 3.3: From wetting to essential discontinuity. Here $L_{1}=o(L)$ and $L N^{1-\alpha}=o(1)$. 


\subsection{Uncountable set of essentially discontinuous points}

Note that, by the proof of Lemma 25 , every configuration $\left(\sigma_{i}\right)_{i \in \mathbb{Z}}$ satisfying

$$
\sup _{\omega}\left|\sum_{i<0} \sum_{j>0} \frac{1}{|i-j|^{\alpha}} \sigma_{i} \omega_{j}\right|<c
$$

for some constant $c>0$ that does not depend on $\sigma$ is an essentially discontinuous point. It is natural to ask how many essentially discontinuous points there are. We will show that, if we reduce the interval of $\alpha$ to be $\left(\frac{3}{2}, 2\right)$, then we have an uncountable set of essentially discontinuous points.

Consider $\nu$ be the i.i.d. a priori measure on $E=\{-1,1\}$ defined by

$$
\nu(X=1)=\nu(X=-1)=\frac{1}{2} .
$$

Let $\left(X_{i}\right)_{i \in \mathbb{Z}}$ be a family of random variables on $(E, \nu)$. For $\omega=\left(\omega_{j}\right)_{j>0}$ be a fixed configuration on $\{-1,1\}^{\mathbb{Z}_{+}}$, define

$$
Y=Y(\omega):=\sum_{i<0} \sum_{j>0} \frac{1}{|i-j|^{\alpha}} X_{i} \omega_{j}
$$

be the "random energy" of the past.

Proposition 6. If $\alpha>3 / 2$, then $\operatorname{Var}(Y)<\infty$.

Proof. Since $\mathbb{E}\left(X_{i}\right)=0$ for every $i \in \mathbb{Z}$, the variance of $Y$ is given by $\operatorname{Var}_{\nu}(Y)=\mathbb{E}\left(Y^{2}\right)$.

$$
\begin{aligned}
Y^{2} & =\left(\sum_{i<0} \sum_{j>0} \frac{1}{|i-j|^{\alpha}} X_{i} \omega_{j}\right)\left(\sum_{l<0} \sum_{k>0} \frac{1}{|l-k|^{\alpha}} X_{l} \omega_{k}\right) \\
& =\sum_{i<0} \sum_{j>0} \sum_{l<0} \sum_{k>0} \frac{1}{|i-j|^{\alpha}} \cdot \frac{1}{|l-k|^{\alpha}} X_{i} X_{l} \omega_{j} \omega_{k} .
\end{aligned}
$$

Thus, since for $i \neq l$ we have $\mathbb{E}_{\nu}\left(X_{i} X_{l}\right)=\mathbb{E}_{\nu}\left(X_{i}\right) \mathbb{E}_{\nu}\left(X_{l}\right)=0$, and $\mathbb{E}_{\nu}\left(X_{i}^{2}\right)=1$ for every $i \in \mathbb{Z}$,

$$
\begin{aligned}
\mathbb{E}_{\nu}\left(Y^{2}\right) & =\sum_{i<0} \sum_{j>0} \sum_{l<0} \sum_{k>0} \frac{1}{|i-j|^{\alpha}} \cdot \frac{1}{|l-k|^{\alpha}} \mathbb{E}\left(X_{i} X_{l}\right) \omega_{j} \omega_{k} \\
& =\sum_{i<0} \sum_{j>0} \sum_{k>0} \frac{1}{|i-j|^{\alpha}} \cdot \frac{1}{|i-k|^{\alpha}} \omega_{j} \omega_{k} .
\end{aligned}
$$

Thus, the variance is bounded by

$$
\begin{aligned}
\operatorname{Var}_{\nu}(Y) & \leq \sum_{i<0} \sum_{j>0} \sum_{k>0} \frac{1}{|i-j|^{\alpha}} \cdot \frac{1}{|i-k|^{\alpha}} \\
& =\sum_{i>0} \sum_{j>0} \frac{1}{(i+j)^{\alpha}} \sum_{k>0} \frac{1}{(i+k)^{\alpha}} .
\end{aligned}
$$

Since $|i+x|^{-\alpha}$ is a decreasing function on $x$, we have

$$
\sum_{k>0} \frac{1}{(i+k)^{\alpha}} \leq \int_{0}^{\infty} \frac{1}{(i+x)^{\alpha}} \mathrm{d} x=\frac{1}{\alpha-1} i^{1-\alpha}
$$

Then,

$$
\operatorname{Var}_{\nu}(Y) \leq \frac{1}{(\alpha-1)^{2}} \sum_{i>0} i^{2-2 \alpha}<\infty
$$


when $2-2 \alpha<-1$, i.e., $\alpha>3 / 2$.

\subsection{Final remarks and open questions}

We have as our main result shown that between the class of Gibbs measures and the class of $g$ measures, neither of them contains the other one. Thus one-sided continuity and two-sided continuity of conditional probabilities are really different properties and there exists a clear distinction between these two notions.

The result on entropic repulsion which we used in the proof presumably can be improved in various respects. We mention a few open questions regarding these issues.

It is not clear to us whether entropic repulsion holds for the case $\alpha=2$. The interface in that case has macroscopic, rather than mesoscopic fluctuations, which makes our proof break down.

Neither is it clear to us whether the methods of Littin and Picco [127] will allow to extend the entropic repulsion results to other $\alpha$ values, although we expect them to hold also in the regime $\alpha \in(1,2]$.

In [22] it is possible to show the phase transition of the Dyson model by using contour argument assuming $J(1)=1$ rather than $J(1) \gg 1$, but reducing the interval of $\alpha$. Since our result should suppose $J(1)$ large enough, we are also interested if it is possible to extend the entropic repulsion result assuming $J(1)=1$.

We give lower bounds for the entropic repulsion, that is the size of the "wet" region but have neither checked if upper bounds are feasible, nor if the entropic repulsion holds all the way up to the critical point.

We showed in Proposition 6 that, reducing the interval of $\alpha$ to $\left(\frac{3}{2}, 2\right]$, there are uncountable number of configurations that the "random energy" of the past is uniformly finite. However, we conjecture that this property should happen for every $\alpha \in(1,2]$. 



\section{Chapter 4}

\section{Stability of the Phase Transition of Critical-Field Ising Model on Cayley Trees}

\subsection{Introduction}

The ferromagnetic Ising model on a Cayley tree has been extensively studied. Some early treatments which were mathematically rigorous can be found in papers by Katsura and Takizawa [111] and by Preston [138]. As opposed to the situation on $\mathbb{Z}^{d}$, the phase transition on Cayley trees can appear even when the model has a non-zero homogeneous external field, because these trees are non-amenable, see [108].

Preston analysed the phase transition using Markov chains. More precisely, he proved that a large class of Gibbs measures can be written as Markov chains, and the set of translational invariant Gibbs measures contains one, two or three completely homogeneous Markov chains. For more details, see [84] and [150].

The main approach to show the phase transition of the Ising model on a Cayley tree uses the fact that we can restrict ourselves to this well-behaved class of Gibbs measures, to which (among others) each extremal Gibbs measure belongs; this is the class of probability measures named "splitting Gibbs measures" or "Markov chains" (see [84, 150] and [120]). The advantage to work with this class of probability measures is the fact that, on Cayley trees, we have a notion of compatibility (see the definition in the next section), which implies that such a probability measure is a Markov chain. By this approach Preston showed that there exists a positive critical value for homogeneous external fields depending on the temperature and the order of the Cayley tree $\Gamma^{d}$, indicated by $h_{c}(\beta, d)$, and a critical temperature, indicated by $\beta_{c}(d)$, such that, if $\beta \leq \beta_{c}(d)$ or $|h|>h_{c}(\beta, d)$, there is no phase transition, and for $\beta>\beta_{c}(d)$ and $|h| \leq h_{c}(\beta, d)$, the model undergoes a phase transition. Moreover, when $|h|=h_{c}(\beta, d)$, there exist exactly two homogeneous splitting Gibbs measures, while for $|h|<h_{c}(\beta, d)$ there exist exactly three homogeneous splitting Gibbs measures.

Technically, the advantage is that the study of Gibbs measures reduces to the study of a set of recurrence equations on "boundary laws" (which we sometimes also call "boundary fields"). This reduction actually does not need any translation invariance; for some earlier work where nontranslation-invariant versions play a role, see e.g. [146], and [57] and in particular the discussion in its Appendix.

When we assume inhomogeneous external fields, we want to consider perturbations of this critical field, with the perturbations decaying to zero when the distance to the root approaches infinity.

We consider this regime, as it is the most sensitive, and presents the closest equivalent of the question considered in [20,21]. We want to look how fast the external fields can decay to the critical value in order to still be able to see a phase transition.

Moreover, we remark that spin systems in decaying fields have been studied to model systems 
in traps, see e.g. [38].

On the lattice $\mathbb{Z}^{d}$, we know by the Peierls argument [135] that in zero field there is a transition for $d \geq 2$. On the other hand, Lee and Yang [125, 153] showed that the Ising model with non-zero homogeneous external fields has uniqueness for any temperature. Thus, the critical value in this case is $h_{c}=0$.

The purpose of this paper is to look at the ferromagnetic Ising model on the Cayley tree $\Gamma^{d}$ with spatially dependent external fields. As in [20], the external fields are decaying to the critical value, which here is non-zero, $h_{c}=h_{c}(\beta, d)$. We will show that, if the external fields are of the form $h_{n}=-h_{c}-\epsilon_{n}$, with $\epsilon_{n}$ positive, decreasing, decaying to zero and satisfying the following condition,

$$
\lim _{n \rightarrow \infty} \sum_{j=1}^{n}\left(\sum_{i=j}^{n} \epsilon_{i}\right)^{2}<\infty
$$

then the model undergoes a phase transition at low temperature. On the other hand, when the condition is violated, and the sum diverges, we will obtain uniqueness of the Gibbs measure for the perturbed model.

Note that the above condition is substantially weaker than the one from [21]. In fact, the condition that we would get from the arguments of [21] is

$$
\sum_{n \geq 1} d^{n} \epsilon_{n}<\infty
$$

It is easy to see that every sequence $\left(\epsilon_{n}\right)_{n \geq 1}$ satisfying (4.2) also satisfies (4.1). We will say that the phase transition persists if the maximal measure $\mu^{+}$is different from the minimal measure $\mu^{-}$. We will consider in particular the persistence of the plus state (that is the positively magnetised state) in a negative critical field. We notice that this state is unstable (disappears) if we add a homogeneous negative external field; we ask the question what happens once we add a negative field decaying to zero. If the decay is fast the transition persists, if the decay is slow enough it may disappear. The condition mentioned above indicates the threshold between those two behaviours.

Some other aspects of the Ising model in a critical field have been studied by Bleher et al [25]. The stability for decaying interactions of the Ising model in non-critical fields, and also for summable inhomogeneous fields as in [21], has been considered by Ganikhodjaev [83].

\subsubsection{Splitting Gibbs Measures}

Let $\Gamma^{d}=(V, L)$ be the Cayley tree of order $d$, i.e., a $d+1$-regular infinite tree. For a fixed $x_{0} \in V$, called the root, define generation $n$ by $W_{n}=\left\{x \in V: \mathrm{d}\left(x, x_{0}\right)=n\right\}$ and $V_{n}=\cup_{k=0}^{n} W_{k}$. For each $x \in W_{n}$, denote $S(x)=\left\{y \in W_{n+1}: \mathrm{d}(x, y)=1\right\}$ for the set of children of $x$. Define also $L_{n}$ to be the edges of the subtree of $\Gamma^{d}$ restricted to the vertices $V_{n}$. For each $x \in V$, we denote the distance of $x$ from the root $x_{0}$ by $\|x\|=\mathrm{d}\left(x, x_{0}\right)$.

Let $\sigma \in \Omega$ and $J>0$. We will recall the definition of the Hamiltonian of the ferromagnetic Ising model on the volume $V_{n}$,

$$
H_{n}^{0}(\sigma)=-J \sum_{\langle x, y\rangle \in L_{n}} \sigma_{x} \sigma_{y} .
$$

Let $h_{n} \in \mathbb{R}$ for $n \geq 0$ and $b_{x} \in \mathbb{R}$ for each $x \in V$. The $b_{x}$ will be the boundary fields, and often we will write $b_{n}$, in the situation where the boundary fields only depend on the generation $n$. We define the probability measure $\mu_{n}$ on the volume $V_{n}$ as

$$
\mu_{n}(\sigma)=\frac{1}{Z_{n}} \exp \left\{-\beta H_{n}^{0}(\sigma)+\sum_{k=0}^{n-1} \sum_{x \in W_{k}} h_{k} \sigma_{x}+\sum_{x \in W_{n}} b_{x} \sigma_{x}\right\},
$$


where $\beta$ is the inverse temperature, and $Z_{n}$ is the partition function given by

$$
Z_{n}=\sum_{\sigma_{V_{n}} \in \Omega_{V_{n}}} \exp \left\{-\beta H_{n}^{0}(\sigma)+\sum_{k=0}^{n-1} \sum_{x \in W_{k}} h_{k} \sigma_{x}+\sum_{x \in W_{n}} b_{x} \sigma_{x}\right\} .
$$

We say that the probability measures $\mu_{n}$ are compatible if for all $n \geq 1$ and $\sigma_{V_{n-1}} \in \Omega_{V_{n-1}}$ :

$$
\sum_{\omega \in \Omega_{W_{n}}} \mu_{n}\left(\sigma_{V_{n-1}} \omega_{W_{n}}\right)=\mu_{n-1}\left(\sigma_{V_{n-1}}\right) .
$$

Here $\sigma_{V_{n-1}} \omega_{W_{n}}$ is the concatenation of the configurations.

Under condition (4.6), by Kolmogorov's Theorem, there exists a unique measure $\mu$ on $\Omega$ such that, for all $n$ and $\sigma_{n} \in \Omega_{V_{n}}$,

$$
\mu\left(\left.\sigma\right|_{V_{n}}=\sigma_{n}\right)=\mu_{n}\left(\sigma_{n}\right) .
$$

This measure is called a splitting Gibbs measure (For Kolmogorov's Theorem, see [158]).

By [84], we know that the Gibbs measures with boundary condition plus and minus are extremal, and all extremal Gibbs measures are splitting Gibbs measures. The free-boundary Gibbs measure $\mu^{\sharp}$ at sufficiently low temperatures is not extremal, but still a splitting Gibbs measure. Taking an arbitrary convex mixture of those three states in general will give a non-splitting measure, however ${ }^{1}$.

\subsection{Compatibility}

From now on, we will assume that $b_{x}$ for all $x \in V$ is such that $b_{x}=b(\|x\|)$, i.e., $b_{x}$ depends only on the distance of $x$ from the root. Writing $b_{n}=b_{x}$ when $\|x\|=n$, we will usually also assume that $b_{n}$ is decreasing. The following result is adapted from Rozikov ([150] Theorem 2.1), who treated general inhomogeneous fields.

Theorem 28. The probability measures $\left(\mu_{n}\right)_{n \geq 1}$ are compatible if and only if for every $n \geq 2$ the following equation holds,

$$
b_{n-1}=h_{n-1}+d F\left(b_{n}, \theta\right),
$$

where $\theta=\tanh (\beta J)$ and $F(x, \theta)=\operatorname{arctanh}(\theta \tanh x)$.

Proof. Suppose that (4.6) holds. Substituting in the probability measure (4.4), we obtain, by multiplying both left-hand side and right-hand side of (4.4) by $Z_{n-1}$,

$$
\begin{aligned}
& \frac{Z_{n-1}}{Z_{n}} \sum_{\omega \in \Omega_{W_{n}}} \exp \left\{-\beta H_{n-1}^{0}(\sigma)+\beta J \sum_{x \in W_{n-1}} \sum_{y \in S(x)} \sigma_{x} \omega_{y}+\sum_{k=0}^{n-1} \sum_{x \in W_{k}} h_{k} \sigma_{x}+b_{n} \sum_{x \in W_{n}} \omega_{x}\right\} \\
& =\exp \left\{-\beta H_{n-1}^{0}(\sigma)+\sum_{k=0}^{n-2} \sum_{x \in W_{k}} h_{k} \sigma_{x}+b_{n-1} \sum_{x \in W_{n-1}} \sigma_{x}\right\} .
\end{aligned}
$$

This implies the following equation,

$$
\frac{Z_{n-1}}{Z_{n}} \sum_{\omega \in \Omega_{W_{n}}} \exp \left\{\sum_{x \in W_{n-1}} \sum_{y \in S(x)}\left(\beta J \sigma_{x} \omega_{y}+b_{n} \omega_{y}\right)\right\}=\exp \left\{\left(b_{n-1}-h_{n-1}\right) \sum_{x \in W_{n-1}} \sigma_{x}\right\} .
$$

\footnotetext{
${ }^{1}$ There is an active interest under which conditions the free-boundary measure, and its analogues in external fields, for Potts models etc, are extremal. This is also known as the "reconstruction problem". See e.g. [60, 71, 131]. However, for the present work this question plays no role.
} 
Thus,

$$
\frac{Z_{n-1}}{Z_{n}} \sum_{\omega \in \Omega_{W_{n}}} \prod_{x \in W_{n-1}} \prod_{y \in S(x)} \exp \left\{\beta J \sigma_{x} \omega_{y}+b_{n} \omega_{y}\right\}=\prod_{x \in W_{n-1}} \exp \left\{\left(b_{n-1}-h_{n-1}\right) \sigma_{x}\right\},
$$

and therefore,

$$
\frac{Z_{n-1}}{Z_{n}} \prod_{x \in W_{n-1}}\left(\sum_{u \in\{-1,1\}} \exp \left\{\beta J \sigma_{x} u+b_{n} u\right\}\right)^{d}=\prod_{x \in W_{n-1}} \exp \left\{\left(b_{n-1}-h_{n-1}\right) \sigma_{x}\right\} .
$$

Substituting in the last equality $\sigma_{x}=1$ and $\sigma_{x}=-1$ for all $x \in V$, and dividing the first expression by the second one, we obtain

$$
\left(\frac{\sum_{u \in\{-1,1\}} \exp \left\{\beta J u+b_{n} u\right\}}{\sum_{u \in\{-1,1\}} \exp \left\{-\beta J u+b_{n} u\right\}}\right)^{d}=\exp \left\{2\left(b_{n-1}-h_{n-1}\right)\right\}
$$

Taking logarithms, we get the desired formula. Note that

$$
F(x, \theta)=\operatorname{arctanh}(\theta \tanh x)=\frac{1}{2} \log \frac{(1+\theta) e^{2 x}+(1-\theta)}{(1-\theta) e^{2 x}+(1+\theta)} .
$$

For the converse, note that

$$
\begin{aligned}
& \sum_{\omega \in \Omega_{W_{n}}} \mu_{n}\left(\sigma_{V_{n-1}} \omega_{W_{n}}\right) \\
& =\frac{1}{Z_{n}} \exp \left\{-\beta H_{n-1}^{0}(\sigma)+\sum_{k=0}^{n-1} \sum_{x \in W_{k}} h_{k} \sigma_{x}\right\} \prod_{x \in W_{n-1}}\left(\sum_{u \in\{-1,1\}} \exp \left\{\beta J \sigma_{x} u+b_{n} u\right\}\right)^{d} .
\end{aligned}
$$

For any $t \in\{-1,1\}$, we have the following identity,

$$
\left(\sum_{u \in\{-1,1\}} \exp \left\{\beta J t u+b_{n} u\right\}\right)^{d}=a_{n} \exp \left\{t\left(b_{n-1}-h_{n-1}\right)\right\}
$$

for some $a_{n}>0$. Consider the function $A_{n}=a_{n}^{s}$ where $s=\left|W_{n-1}\right|$. We have

$$
\begin{aligned}
& \sum_{\omega \in \Omega_{W_{n}}} \mu_{n}\left(\sigma_{V_{n-1}} \omega_{W_{n}}\right) \\
= & \frac{A_{n}}{Z_{n}} \exp \left\{-\beta H_{n-1}^{0}(\sigma)+\sum_{k=0}^{n-1} \sum_{x \in W_{k}} h_{k} \sigma_{x}\right\} \prod_{x \in W_{n-1}} \exp \left\{\sigma_{x}\left(b_{n-1}-h_{n-1}\right)\right\} .
\end{aligned}
$$

Using the fact that $\sum_{\sigma \in \Omega_{V_{n-1}}} \sum_{\omega \in \Omega_{W_{n}}} \mu_{n}\left(\sigma_{V_{n-1}} \omega_{W_{n}}\right)=1$, we have $A_{n}=Z_{n} / Z_{n-1}$.

By Theorem 28, there is a bijection between sets $\mathbf{b}=\left\{b_{n}, n \geq 1\right\}$ satisfying equation (4.8) and splitting Gibbs measures $\mu$. Thus, in particular, the extremal Gibbs measures $\mu_{\beta, \bar{h}}^{ \pm}$are associated to the boundary fields $\tilde{\mathbf{b}}^{ \pm}=\left\{\tilde{b}_{n}^{ \pm}, n \geq 1\right\}$.

The homogeneous splitting Gibbs measures for the Ising model in homogeneous fields, i.e., $h_{n}=h$ for all $n \geq 0$, are very well known, see e.g. [84] and [150]. The translation-invariant solutions $\left(b_{n}\right)_{n \geq 1}$ to the recurrence equation (4.8) when $h_{n}=h$ for all $n \geq 0$ are constant, i.e., $b_{n}=b^{*}$ for all 
$n \geq 1$. Thus, we have the equation

$$
b^{*}=h+d F\left(b^{*}, \theta\right):=\psi_{h}\left(b^{*}\right)
$$

where $\psi_{h}(x)=h+d F(x, \theta)$.

By [84], [150] we know that there exists $\beta_{c}(d)>0$ and $h_{c}(\beta, d)>0$ such that:

(1) If $\beta \leq \beta_{c}(d)$ or $|h|>h_{c}(\beta, d)$, the function $\psi_{h}$ has exactly one fixed point. We define the solution as the sequence $\mathbf{b}^{\sharp}=\left\{b_{n}^{\sharp}\right\}_{n \geq 1}$ such that $b_{n}^{\sharp}=b^{\sharp}$ is constant.

(2) If $\beta>\beta_{c}(d)$ and $|h|<h_{c}(\beta, d)$, the function $\psi_{h}$ has exactly three fixed points. The solutions are the sequences $\mathbf{b}^{\sharp}, \mathbf{b}^{+}=\left\{b_{n}^{+}\right\}_{n \geq 1}$ and $\mathbf{b}^{-}=\left\{b_{n}^{-}\right\}_{n \geq 1}$ in which $b_{n}^{+}=b^{+}$and $b_{n}^{-}=b^{-}$with $b^{-}<b^{+}$are constant for all $n \geq 1$.

(3) If $\beta>\beta_{c}(d)$ and $|h|=h_{c}(\beta, d)$, the function $\psi_{h}$ has exactly two fixed points. For $h=h_{c}$ the sequences $\mathbf{b}^{\sharp}$ and $\mathbf{b}^{-}$coincide, and for $h=-h_{c}$ the sequences $\mathbf{b}^{\sharp}$ and $\mathbf{b}^{+}$coincide.

We have the following properties of the function $F(x, \theta)$.

1. $F(-x, \theta)=-F(x, \theta)$.

2. $\lim _{x \rightarrow \infty} F(x, \theta)=\operatorname{arctanh}(\theta)$.

3. $\left.\frac{\mathrm{d}}{\mathrm{d} x} F(x, \theta)\right|_{x=0}=\theta$, and $0 \leq \frac{\mathrm{d}}{\mathrm{d} x} F(x, \theta) \leq \theta$.

4. $\frac{\mathrm{d}^{2}}{\mathrm{~d} x^{2}} F(x, \theta)<0$ for every $x>0$.

Note that $b^{+}$is a saddle node of $\psi:=\psi_{-h_{c}}$, i.e., $\psi^{\prime}\left(b^{+}\right)=1$; this means that $\mathbf{b}^{+}$is (marginally) stable in a minus field. It attracts higher values, but repels lower ones. The "stable" $\mathbf{b}^{-}$is an attractor of all initial $b$ below $b^{+}$. In fact it attracts exponentially fast, due to the map $\psi(b)-b$ near $b^{-}$being contracting.

Also, we note that similarly, in a positive critical field, $b^{-}$is the saddle node of $\psi_{h}$ for $h=h_{c}$, and thus $\mathbf{b}^{-}$is marginally stable and $\mathbf{b}^{+}$is the stable attractor in a plus field.

For every $h \in \mathbb{R}$, let

$$
\begin{aligned}
\psi_{h}(\infty) & :=\lim _{x \rightarrow \infty} \psi_{h}(x)=h+d \beta J, \\
\psi_{h}(-\infty) & :=\lim _{x \rightarrow-\infty} \psi_{h}(x)=h-d \beta J .
\end{aligned}
$$

Proposition 7. Let $J>0$ and $h_{n}=h$ for every $n \geq 1$. If $\left(b_{n}\right)_{n \geq 1}$ is a solution to (4.8), then

$$
b^{-} \leq b_{n} \leq b^{+} \quad \text { for every } n \geq 1 \text {. }
$$

Proof. Note that, for every $b \in \mathbb{R}$,

$$
-h_{c}+d \lim _{x \rightarrow-\infty} F(x, \theta) \leq-h_{c}+d F(b, \theta) \leq-h_{c}+d \lim _{x \rightarrow \infty} F(x, \theta) .
$$

Then,

$$
\psi_{h}(-\infty) \leq \psi_{h}(b) \leq \psi_{h}(\infty) .
$$

For every $n \geq 1$ and $k \geq 1$, we have

$$
\psi_{h}^{k}(-\infty) \leq \psi_{h}^{k}\left(b_{n+k}\right) \leq \psi_{h}^{k}(\infty) .
$$

This implies

$$
\psi_{h}^{k}(-\infty) \leq b_{n} \leq \psi_{h}^{k}(\infty) .
$$

By (4.12) we have $\psi_{h}^{n+1}(\infty) \leq \psi_{h}^{n}(\infty)$ for every $n \geq 1$, i.e., the sequence $\psi_{h}^{n}(\infty)$ is decreasing. Since $\psi_{h}(\infty) \geq b^{+}$and $b^{+}$is a fixed point of $\psi_{h}$, we have $\psi_{h}^{n}(\infty) \geq b^{+}$for every $n \geq 1$. Thus, the sequence 
$\psi_{h}^{n}(\infty)$ has limit equals to $L$, and $L \geq b^{+}$. Since $L$ is a fixed point of $\psi_{h}$, and there is no fixed point in $\left(b^{+}, \infty\right)$, we conclude that $L=b^{+}$. The proof that the limit of $\psi_{h}^{n}(-\infty)$ is equal to $b^{-}$is analogous.

Let us also show the inhomogeneous version of Proposition 7.

Proposition 8. Let $J>0$ and $h_{n} \in \mathbb{R}$ for every $n \geq 1$. If $\left(b_{n}\right)_{n \geq 1}$ is a solution to (4.8), then

$$
\tilde{b}_{n}^{-} \leq b_{n} \leq \tilde{b}_{n}^{+} \quad \text { for every } n \geq 1
$$

where the boundary fields $\tilde{\mathbf{b}}^{ \pm}=\left\{\tilde{b}_{n}^{ \pm}, n \geq 1\right\}$ are associated to the extremal Gibbs measures $\mu_{\beta, \bar{h}}^{ \pm}$.

Proof. Let us show that $b_{n} \leq \tilde{b}_{n}^{+}$for every $n \geq 1$. The proof for the inequality $\tilde{b}_{n}^{-} \leq b_{n}$ is analogous. Fix $n \geq 1$. Consider the function

$$
f(\sigma)= \begin{cases}1, & \text { if } \sigma_{x}=1 \text { for every } x \in V_{n} \\ 0, & \text { otherwise }\end{cases}
$$

By FKG inequality,

$$
\begin{aligned}
& \frac{1}{Z_{n}} \sum_{\sigma \in \Omega_{V_{n}}} f(\sigma) \exp \left\{-\beta H_{n}^{0}(\sigma)+\sum_{k=0}^{n-1} \sum_{x \in W_{k}} h_{k} \sigma_{x}+\sum_{x \in W_{n}} b_{x} \sigma_{x}\right\} \\
& \leq \frac{1}{Z_{n}^{+}} \sum_{\sigma \in \Omega_{V_{n}}} f(\sigma) \exp \left\{-\beta H_{n}^{0}(\sigma)+\sum_{k=0}^{n-1} \sum_{x \in W_{k}} h_{k} \sigma_{x}+\sum_{x \in W_{n}} \tilde{b}_{n}^{+} \sigma_{x}\right\},
\end{aligned}
$$

where

$$
Z_{n}^{+}=\sum_{\sigma \in \Omega_{V_{n}}} \exp \left\{-\beta H_{n}^{0}(\sigma)+\sum_{k=0}^{n-1} \sum_{x \in W_{k}} h_{k} \sigma_{x}+\sum_{x \in W_{n}} \tilde{b}_{n}^{+} \sigma_{x}\right\}
$$

Thus,

$$
\exp \left\{\left|W_{n}\right|\left(\tilde{b}_{n}^{+}-b_{n}\right)\right\} \geq \frac{Z_{n}^{+}}{Z_{n}}=\mu_{n}\left(\exp \left\{\left(\tilde{b}_{n}^{+}-b_{n}\right) \sum_{x \in W_{n}} \sigma_{x}\right\}\right),
$$

and the inequality (4.18) holds if, and only if, $\tilde{b}_{n}^{+} \geq b_{n}$, as we desired.

\subsection{Results and proofs}

Our analysis is based on the behavior of the sum $\sum_{j=1}^{n}\left(\sum_{i=j}^{n} \epsilon_{i}\right)^{2}$ for the perturbation of the field $\left(\epsilon_{k}\right)_{k \geq 1}$. Firstly, the following inequality in the next proposition is inspired by the rearrangement inequality and somehow helps to see the behavior of the sum.

Proposition 9. For any decreasing sequence $\left(\epsilon_{n}\right)_{n \geq 1}$ of positive numbers,

$$
\sum_{i=1}^{n}\left(i \epsilon_{i}\right)^{2} \leq \sum_{j=1}^{n}\left(\sum_{i=j}^{n} \epsilon_{i}\right)^{2} \leq \sum_{i=1}^{n}\left((n-i+1) \epsilon_{i}\right)^{2} .
$$

Proof. For the upper bound, using that $\epsilon_{i} \leq \epsilon_{j}$ for any $i \geq j$, we have

$$
\sum_{j=1}^{n}\left(\sum_{i=j}^{n} \epsilon_{i}\right)^{2} \leq \sum_{j=1}^{n}\left(\sum_{i=j}^{n} \epsilon_{j}\right)^{2}=\sum_{i=1}^{n}\left((n-i+1) \epsilon_{i}\right)^{2} .
$$




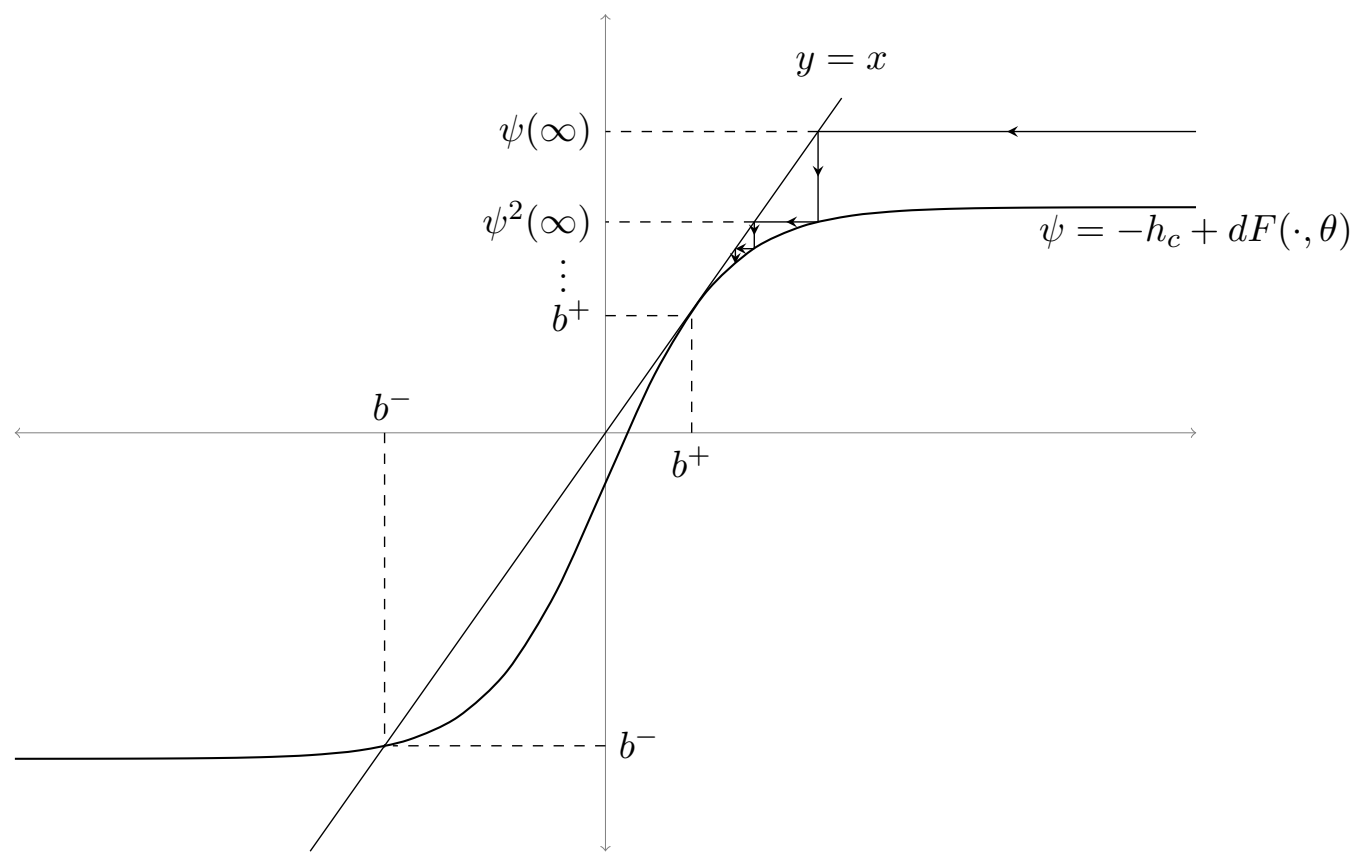

Figure 4.1: The graph of $\psi$ for $h=-h_{c}$ and the fixed points $b^{+}$and $b^{-}$, and the sequence $\psi^{n}(\infty)$ converging to $b^{+}$.

For the lower bound, we use the following expression,

$$
\sum_{j=1}^{n}\left(\sum_{i=j}^{n} \epsilon_{i}\right)^{2}=\sum_{i=1}^{n} i \epsilon_{i}^{2}+2 \sum_{i=2}^{n} \epsilon_{i}\left(\sum_{j=1}^{i-1} j \epsilon_{j}\right) .
$$

Thus,

$$
\sum_{j=1}^{n}\left(\sum_{i=j}^{n} \epsilon_{i}\right)^{2} \geq \sum_{i=1}^{n} i \epsilon_{i}^{2}+2 \sum_{i=2}^{n} \epsilon_{i}^{2}\left(\sum_{j=1}^{i-1} j\right)=\sum_{i=1}^{n}\left(i \epsilon_{i}\right)^{2}
$$

as we wanted.

The results will be based on an estimate of the influence from infinity on boundary fields near the origin. If the influence due to the inhomogeneous fields is small enough, the non-uniqueness of Gibbs measures will not change, if the influence of the inhomogeneous terms gets too big, then we will have a unique Gibbs measure.

The external fields that we are working with are of the form $h_{n}=-h_{c}-\epsilon_{n}$. Consider the function $\tilde{\psi}_{n}(x):=h_{n}+d F(x, \theta)=\psi(x)-\epsilon_{n}$, where $\psi(x)=-h_{c}+d F(x, \theta)$. For each $n \geq 1$ and $k \leq n$, define

$$
\tilde{\psi}_{k, n}(x)= \begin{cases}\tilde{\psi}_{n}(x), & \text { if } k=n, \\ \tilde{\psi}_{k}\left(\tilde{\psi}_{k+1, n}(x)\right), & \text { if } k<n,\end{cases}
$$

i.e., $\tilde{\psi}_{k, n}(x)=\tilde{\psi}_{k} \circ \ldots \circ \tilde{\psi}_{n}(x)$. Define

$$
\begin{aligned}
\tilde{\psi}_{n}(\infty) & :=\lim _{x \rightarrow \infty} \tilde{\psi}_{n}(x)=h_{n}+d \beta J, \\
\tilde{\psi}_{n}(-\infty) & :=\lim _{x \rightarrow-\infty} \tilde{\psi}_{n}(x)=h_{n}-d \beta J .
\end{aligned}
$$

We have the following identity for the boundary fields $\tilde{\mathbf{b}}^{ \pm}$. 
Proposition 10. For every $k \geq 1$,

$$
\lim _{n \rightarrow \infty} \tilde{\psi}_{k, n}(\infty)=\tilde{b}_{k}^{+} \quad \text { and } \quad \lim _{n \rightarrow \infty} \tilde{\psi}_{k, n}(-\infty)=\tilde{b}_{k}^{-} .
$$

Proof. Let $L_{k}=\lim _{n \rightarrow \infty} \tilde{\psi}_{k, n}(\infty)$. We have that $\left(L_{k}\right)_{k \geq 1}$ is a solution of (4.8). In fact,

$$
\begin{aligned}
\tilde{\psi}_{k-1}\left(L_{k}\right) & =\tilde{\psi}_{k-1}\left(\lim _{n \rightarrow \infty} \tilde{\psi}_{k, n}(\infty)\right) \\
& =\lim _{n \rightarrow \infty} \tilde{\psi}_{k-1, n}(\infty) \\
& =L_{k-1} .
\end{aligned}
$$

Then, by Proposition $8, L_{k} \leq \tilde{b}_{k}^{+}$for every $k \geq 1$. Let us show that $\tilde{\psi}_{k, n}(\infty) \geq \tilde{b}_{k}^{+}$for every $n \geq k$. First, we have

$$
\tilde{\psi}_{k}(\infty)=h_{k}+d \lim _{x \rightarrow \infty} F(x, \theta) \geq h_{k}+d F\left(\tilde{b}_{k+1}^{+}, \theta\right)=\tilde{b}_{k}^{+}
$$

for every $k \geq 1$. For $n>k$, since $F$ is an increasing function, we have

$$
\tilde{\psi}_{k, n}(\infty)=\tilde{\psi}_{k, n-1}\left(\tilde{\psi}_{n}(\infty)\right) \geq \tilde{\psi}_{k, n-1}\left(\tilde{b}_{n}^{+}\right)=\tilde{b}_{k}^{+} .
$$

This concludes that $L_{k} \geq \tilde{b}_{k}^{+}$for every $k \geq 1$. The proof for $\lim _{n \rightarrow \infty} \tilde{\psi}_{k, n}(-\infty)=\tilde{b}_{k}^{-}$is analogous.

Note that, for any $k \geq 1$,

$$
\begin{gathered}
\tilde{b}_{k}^{+}=\lim _{n \rightarrow \infty} \tilde{\psi}_{k, n}(\infty) \geq \lim _{n \rightarrow \infty} \tilde{\psi}_{k, n}\left(b^{+}\right), \\
\tilde{b}_{k}^{-}=\lim _{n \rightarrow \infty} \tilde{\psi}_{k, n}(-\infty) \leq \lim _{n \rightarrow \infty} \tilde{\psi}_{k, n}\left(b^{-}\right) .
\end{gathered}
$$

Theorem 29. Consider the ferromagnetic Ising model on a Cayley tree $\Gamma^{d}$ with external fields $\left(-h_{c}-\epsilon_{n}\right)_{n \geq 1}$, where the sequence $\left(\epsilon_{n}\right)_{n \geq 1}$ of positive numbers decreases to zero. Suppose that $\left(\epsilon_{n}\right)_{n \geq 1}$ satisfies the following condition,

$$
\lim _{n \rightarrow \infty} \sum_{j=1}^{n}\left(\sum_{i=j}^{n} \epsilon_{i}\right)^{2}<\infty .
$$

Then the perturbed model undergoes a phase transition.

Proof. Suppose that $\beta>\beta_{c}(d)$, which implies that we have phase transition for the Ising model with external field $-h_{c}$, and let $b^{-}<b^{+}$be the solutions of the equation (4.9). Remind that $b^{+}$is a saddle node, i.e., $\psi^{\prime}\left(b^{+}\right)=1$.

By Taylor expansion on $\psi$, we have

$$
\psi\left(b^{+}-\epsilon_{n}\right)=\psi\left(b^{+}\right)-\psi^{\prime}\left(b^{+}\right) \epsilon_{n}+\frac{1}{2} \psi^{\prime \prime}\left(b^{+}\right) \epsilon_{n}^{2}+O\left(\epsilon_{n}\right)^{3} .
$$

Using the fact that $\psi$ is a concave function around $b^{+}$, we get

$$
\psi\left(b^{+}-\epsilon_{n}\right)=b^{+}-\epsilon_{n}-\frac{1}{2}\left|\psi^{\prime \prime}\left(b^{+}\right)\right| \epsilon_{n}^{2}+O\left(\epsilon_{n}\right)^{3} .
$$

Now, if we apply $\psi$ on $\psi\left(b^{+}-\epsilon_{n}\right)-\epsilon_{n-1}$, we have

$$
\psi\left(\psi\left(b^{+}-\epsilon_{n}\right)-\epsilon_{n-1}\right)=\psi\left(b^{+}-\epsilon_{n}-\epsilon_{n-1}-\frac{1}{2}\left|\psi^{\prime \prime}\left(b^{+}\right)\right| \epsilon_{n}^{2}+O\left(\epsilon_{n}\right)^{3}\right)
$$




$$
\begin{aligned}
& =b^{+}-\left(\epsilon_{n}+\epsilon_{n-1}\right)-\frac{1}{2}\left|\psi^{\prime \prime}\left(b^{+}\right)\right| \epsilon_{n}^{2}-\frac{1}{2}\left|\psi^{\prime \prime}\left(b^{+}\right)\right|\left(\epsilon_{n}+\epsilon_{n-1}+\frac{1}{2}\left|\psi^{\prime \prime}\left(b^{+}\right)\right| \epsilon_{n}^{2}\right)^{2}+O\left(\epsilon_{n-1}\right)^{3} \\
& =b^{+}-\left(\epsilon_{n}+\epsilon_{n-1}\right)-\frac{1}{2}\left|\psi^{\prime \prime}\left(b^{+}\right)\right|\left(\epsilon_{n}^{2}+\left(\epsilon_{n}+\epsilon_{n-1}\right)^{2}\right)+O\left(\epsilon_{n-1}\right)^{3} .
\end{aligned}
$$

Thus, by induction, we obtain our main formula:

$$
\tilde{\psi}_{k, n}\left(b^{+}\right)=b^{+}-\sum_{i=k}^{n} \epsilon_{i}-\frac{1}{2}\left|\psi^{\prime \prime}\left(b^{+}\right)\right| \sum_{i=k+1}^{n}\left(\sum_{j=i}^{n} \epsilon_{j}\right)^{2}+O\left(\epsilon_{k+1}\right)^{3} .
$$

Since the sequence $\left(\epsilon_{n}\right)_{n \geq 1}$ satisfies (4.28), for any $\varepsilon>0$, there exists $k_{1} \geq 1$ such that, for all $k \geq k_{1}$,

$$
\sum_{j=k+1}^{n}\left(\sum_{i=j}^{n} \epsilon_{i}\right)^{2}<\frac{\varepsilon}{\left|\psi^{\prime \prime}\left(b^{+}\right)\right|}
$$

for every $n \geq k$. Moreover, $\left(\epsilon_{n}\right)_{n \geq 1}$ is summable. Thus, there exists $k_{2} \geq 1$ such that, for all $k \geq k_{2}$,

$$
\sum_{i=k}^{n} \epsilon_{i}<\frac{\varepsilon}{2}
$$

for every $n \geq k$. Thus, for all $k \geq \max \left\{k_{1}, k_{2}\right\}$,

$$
\tilde{\psi}_{k, n}\left(b^{+}\right)>b^{+}-\varepsilon+O\left(\epsilon_{k+1}\right)^{3} .
$$

for every $n \geq k$. Let us take $\varepsilon>0$ sufficiently small such that $b^{+}-\varepsilon>b^{-}$. From (4.30), there exists $k_{0} \geq \max \left\{k_{1}, k_{2}\right\}$ and $\delta=\delta(k)>0$ such that, for all $k \geq k_{0}$,

$$
\tilde{\psi}_{k, n}\left(b^{+}\right)>b^{+}-\delta
$$

for every $n>k$. Thus, by (4.26), $\tilde{b}_{k}^{+}>b^{+}-\delta>b^{-}$for all $k \geq k_{0}$. Note also that $b^{-} \geq \tilde{b}_{k}^{-}$, as $b^{-}$ is even stable for perturbation under homogeneous fields, so much the more it is for $\tilde{\mathbf{b}}^{-}$. Therefore $\tilde{b}_{k}^{+}>\tilde{b}_{k}^{-}$for all $k \geq k_{0}$. Since $\tilde{\mathbf{b}}^{+}$and $\tilde{\mathbf{b}}^{-}$are associated to extremal Gibbs measures, namely the Gibbs measures with plus and minus-boundary condition, these measures are distinct.

It is easy to see that the above result also works when we will consider the stability of the minus state in a plus field, under addition of a positive spatially dependent perturbation $\left(\epsilon_{n}\right)_{n \geq 1}$. In that case, we consider the ferromagnetic Ising model on a Cayley tree $\Gamma^{d}$ with external fields $\left(h_{c}+\epsilon_{n}\right)_{n \geq 1}$.

Our second result says that if the inhomogeneous field is negative and such that it does not satisfy the above condition, it is strong enough to remove the phase transition, and indeed there will be one single Gibbs measure.

Theorem 30. Consider the ferromagnetic Ising model on a Cayley tree $\Gamma^{d}$ with external fields $\left(-h_{c}-\epsilon_{n}\right)_{n \geq 1}$, where the sequence $\left(\epsilon_{n}\right)_{n \geq 1}$ of positive numbers decreases to zero. Suppose that $\left(\epsilon_{n}\right)_{n \geq 1}$ satisfies the following condition,

$$
\lim _{n \rightarrow \infty} \sum_{j=1}^{n}\left(\sum_{i=j}^{n} \epsilon_{i}\right)^{2}=\infty
$$

Then the perturbed model has uniqueness for any temperature. 
Proof. For $1 \leq k<n<N$, let us consider the auxiliary boundary fields $\left(b_{m}^{+, k, n, N}\right)_{1 \leq m \leq N}$ in which

$$
b_{m-1}^{+, k, n, N}= \begin{cases}+\infty, & \text { if } m=N+1, \\ \psi\left(b_{m}^{+, k, n, N}\right), & \text { if } n<m \leq N \\ \tilde{\psi}_{m-1}\left(b_{m}^{+, k, n, N}\right), & \text { if } k<m \leq n,\end{cases}
$$

and $b_{m}^{+, k, n, N}$ satisfies the compatibility equation (4.8) for $m \leq k$. This sequence means that we are taking plus boundary condition at distance $N$ from the origin, between $n$ and $N$ the sequence is in the homogeneous case, and between $k$ and $n$ the sequence is in the inhomogeneous case. Note that this provides us with an upper bound for $\tilde{\mathbf{b}}^{+}$.

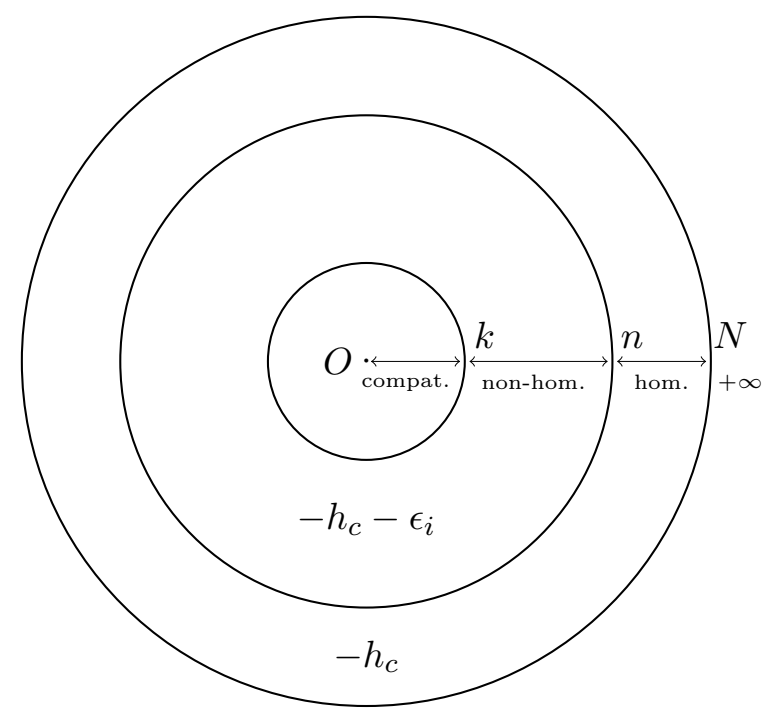

Figure 4.2: The Cayley tree with root $O$, and the auxiliary boundary fields. The circle means the depth of the tree.

It is easy to see that $b_{n}^{+, k, n, N}=\psi^{N-n}(\infty)$. In order for all Taylor expansions in (4.30) to hold, for a fixed $\varepsilon>0$, there exist $N \geq 1$ sufficiently large and $n \leq N$ such that $b^{+}<b_{n}^{+, k, n, N}<b^{+}+\varepsilon$, since $\psi^{p}(\infty)$ converges to $b^{+}$as $p \rightarrow \infty$. Note that, for a larger $N$, we can increase $n$ as well. Thus, taking the limit in $N$, we have $\lim _{N \rightarrow \infty} b_{n}^{+, k, n, N}=b^{+}$. So, we can consider the sequence $\left(b_{m}^{+, k, n}\right)_{m \geq 1}$ defined on the whole Cayley tree by

$$
b_{m-1}^{+, k, n}= \begin{cases}b^{+}, & \text {if } m \geq n+1 \\ \tilde{\psi}_{m-1}\left(b_{m}^{+, k, n}\right), & \text { if } 1<m \leq n\end{cases}
$$

For a fixed $\delta>0$, there exists $k \leq n$ such that

$$
\sum_{j=k+1}^{n}\left(\sum_{i=j}^{n} \epsilon_{i}\right)^{2}>\frac{2 \delta}{\left|\psi^{\prime \prime}\left(b^{+}\right)\right|} .
$$

By (4.30), we thus have $\tilde{\psi}_{k, n}\left(b^{+}\right)<b^{+}-\delta+O\left(\epsilon_{k+1}\right)^{3}+O(\varepsilon)$. Consider $\bar{b}<0$ such that $\psi^{\prime}(\bar{b})=1$. Note that, for every $x<\bar{b}$, we have $0<\psi^{\prime}(x)<1$. Let us choose $\delta>b^{+}-\bar{b}$ and $k$ very large (and thus also $\mathrm{n}$ is large) such that $\epsilon_{k}>0$ is small enough, and $\varepsilon>0$ small enough, in which case there exists $\delta^{\prime}>b^{+}-\bar{b}$ satisfying

$$
b_{k}^{+, k, n}<b^{+}-\delta^{\prime}<\bar{b} .
$$

Note that $\delta^{\prime}$ does not depend on $k$ in the sense that $\delta^{\prime}$ does not change when $k$ increases, once $n$ is large enough, satisfying (4.38). The bound (4.39) means that the perturbation of the external 
fields $\left(\epsilon_{k}\right)_{k>1}$ is strong enough in the sense of condition (4.35) for the boundary field $b_{m}^{+, k, n}$ to be strictly below $\bar{b}$ for every $m \leq k$. Choose $k_{1}$ such that, for every $k \geq k_{1}$, we have the inequality (4.39). Thus, by the fact that $\psi^{\prime}(x)$ is an increasing function for $x<\overline{\bar{b}}$, we have

$$
0<\psi^{\prime}\left(b_{k}^{+, k, n}\right)<\psi^{\prime}\left(b_{k_{1}}^{+, k_{1}, n}\right)=1-\delta^{\prime \prime}
$$

where $\delta^{\prime \prime}:=1-\psi^{\prime}\left(b_{k_{1}}^{+, k_{1}, n}\right)>0$. So, for any $m \leq k$, we have

$$
0<\psi^{\prime}\left(b_{m}^{+, k, n}\right)<1-\delta^{\prime \prime}
$$

Note that $\delta^{\prime \prime}$ does not change when $k$ increases. Thus, for every $k_{0} \geq 1$, there exist $k>\max \left\{k_{0}, k_{1}\right\}$ and $n_{k}>k$ such that (4.39) and (4.40) hold. Note that

$$
\tilde{b}_{k_{0}}^{+}=\lim _{k \rightarrow \infty} b_{k_{0}}^{+, k, n_{k}}
$$

Now, define the boundary fields $\left(b_{m}^{-, n, N}\right)_{1 \leq m \leq N}$ in which

$$
b_{m-1}^{-, n, N}= \begin{cases}-\infty, & \text { if } m=N+1 \\ \psi_{m-1}\left(b_{m}^{-, n, N}\right), & \text { if } n<m \leq N \\ \tilde{\psi}_{m-1}\left(b_{m}^{-, n, N}\right), & \text { if } 1<m \leq n\end{cases}
$$

For a fixed $n<N$, taking $N$ to infinity, we have $\lim _{N \rightarrow \infty} b_{n}^{-, N}=b^{-}$. Thus, we can consider the sequence $\left(b_{m}^{-, n}\right)_{m \geq 1}$ defined in the whole Cayley tree so that

$$
b_{m-1}^{-, n}= \begin{cases}b^{-}, & \text {if } m \geq n+1, \\ \tilde{\psi}_{m-1}\left(b_{m}^{-, n}\right), & \text { if } 1<m \leq n .\end{cases}
$$

Note that

$$
\tilde{b}_{k_{0}}^{-}=\lim _{n \rightarrow \infty} b_{k_{0}}^{-, n}=\lim _{n \rightarrow \infty} \tilde{\psi}_{k_{0}, n-1}\left(b^{-}\right)
$$

for all $k_{0} \geq 1$. The Taylor expansion below shows that these boundary fields decay exponentially by contraction, since $0<\psi^{\prime}\left(b^{-}\right)<1$. In fact,

$$
\tilde{\psi}_{k, n-1}\left(b^{-}\right)=b^{-}-\sum_{i=k}^{n-1} \psi^{\prime}\left(b^{-}\right)^{i-k} \epsilon_{i}+O\left(\epsilon_{k+1}\right)^{2} .
$$

Since the map $\psi$, and similarly $\tilde{\psi}_{m}$, act as contractions on the interval $\left(-\infty, b^{+}-\delta^{\prime}\right)$, with a uniform contraction bound $1-\delta^{\prime \prime}$, we have that in the limit $k$ to infinity the difference in influence on the boundary field at sites $k_{0}$ between negative boundary fields and positive boundary fields less than $b^{+}-\delta^{\prime}$ at sites at distance $k_{0}$ disappears. In fact, we know that the sequence $b_{k}^{-, n}$ converges to $b^{-}$as $k$ is going to infinity (and so $n$ goes to infinity), since $b_{k}^{-, n_{k}} \leq b_{k}^{+, k, n_{k}} \leq b^{+}{ }^{-} \delta^{\prime}$, we have that $\left|b_{k}^{+, k, n_{k}}-b_{k}^{-, n_{k}}\right|<C$ for some $C>0$. Note that $C$ does not depend on $k$. Thus, by the Mean Value Theorem,

$$
\begin{aligned}
\tilde{b}_{k_{0}}^{+}-\tilde{b}_{k_{0}}^{-} & =\lim _{k \rightarrow \infty} b_{k_{0}}^{+, k, n_{k}}-\lim _{n \rightarrow \infty} b_{k_{0}}^{-, n} \\
& =\lim _{k \rightarrow \infty} \tilde{\psi}_{k_{0}, k-1}\left(b_{k}^{+, k, n_{k}}\right)-\lim _{k \rightarrow \infty} \tilde{\psi}_{k_{0}, k-1}\left(b_{k}^{-, n_{k}}\right) \\
& =\lim _{k \rightarrow \infty}\left(\tilde{\psi}_{k_{0}, k-1}\left(b_{k}^{+, k, n_{k}}\right)-\tilde{\psi}_{k_{0}, k-1}\left(b_{k}^{-,, n_{k}}\right)\right) \\
& \leq \lim _{k \rightarrow \infty}\left(\sup _{c \in\left[b_{k}^{\left.-, n_{k}, b_{k}^{+, k, n_{k}}\right]}\right.} \tilde{\psi}_{k_{0}, k-1}^{\prime}(c)\left|b_{k}^{+, k, n_{k}}-b_{k}^{-, n_{k}}\right|\right)
\end{aligned}
$$




$$
\begin{aligned}
& \leq C \lim _{k \rightarrow \infty}\left(1-\delta^{\prime \prime}\right)^{k-k_{0}} \\
& =0
\end{aligned}
$$

Thus, the sequences $\tilde{\mathbf{b}}^{+}$and $\tilde{\mathbf{b}}^{-}$are equal. Therefore the extremal Gibbs measures associated to these sequences, $\mu_{\beta, \bar{h}}^{+}$and $\mu_{\beta, \bar{h}}^{-}$respectively, are equal for any $\beta>0$.

Example. As in $[20,45]$, let us consider the sequence $\epsilon_{k}=1 / k^{\gamma}$, where $\gamma>0$. Note that

$$
\sum_{j=1}^{n}\left(\sum_{i=j}^{n} i^{-\gamma}\right)^{2}=\sum_{j=1}^{n} O\left(j^{2-2 \gamma}\right)
$$

Thus, the sum (4.47) converges when $2-2 \gamma<-1$ and diverges when $2-2 \gamma \geq-1$, and we conclude that the critical power is $\gamma_{c}=3 / 2$. Note also that the model has uniqueness at the critical power, since the sum diverges.

\subsection{Final remarks and open questions}

Our results hold for Cayley trees. One might of course ask similar questions for more general trees, such as, for example, the class considered in [128]. However, even in the situation of constant field, it is so far not clear to us which conditions on the tree would be needed to conclude whether there is a unique or a non-unique Gibbs measure in a critical field. Whether a related kind of perturbation analysis might help to elucidate this, remains to be seen. 


\section{Chapter 5}

\section{Counting Contours on Trees}

\section{$5.1 \quad$ Introduction}

After the seminal paper of Rudolf Peierls [135], the standard technique to prove the existence of phase transitions in spin systems (Ising model type, for instance) goes by a contour argument. Roughly speaking, we need to define objects usually called contours, notions of size (length or surface) and interior for these objects. Furthermore, for a fixed vertex $x$ of a graph $G$ and, for each $n \in \mathbb{N}$, we need to estimate the number of contours of size $n$ in $G$ with $x$ in their interiors.

A standard calculation in this approach is to control expressions as below:

$$
\sum_{C \odot x} w(|C|)=\sum_{n=1}^{\infty} \sum_{\substack{C \odot x \\|C|=n}} w(|C|)=\sum_{n=1}^{\infty} w(n) \sum_{\substack{C \odot x \\|C|=n}} 1,
$$

where $|C|$ denotes the size of the contour $C$ and $C \odot x$ denotes the fact that $x$ belongs to the interior of $C$. Usually the function $w:\{$ contours $\} \rightarrow \mathbb{R}^{+}$depends only on the size of the contour and not on its position in the graph $G$. For the standard Ising model on $\mathbb{Z}^{2}$, the function is given by $w(C)=w(|C|)=\exp (-2 \beta|C|)$ where $\beta$ is the inverse of the temperature. Then, to control (5.1) we need to estimate $\sum_{\substack{C|=n\\| C \mid}} 1$ and for this purpose generating functions are very powerful tools. We can find similar expressions to (5.1) in almost all papers using the Peierls argument. The readers interested in the proof of the existence of phase transitions using the Peierls contours can check standard books in the field $[28,84,142,154]$. The original Peierls argument [135] was done for the Ising model on $\mathbb{Z}^{2}$, but we can define contours for any $\mathbb{Z}^{d}$ with $d \geq 3$ and the argument works as well. The estimates of the number of contours help us to give bounds for the critical temperature of the models, see $[12,123]$. These facts show that the mathematical problem of counting contours on graphs has important consequences in statistical physics and naturally emerges.

Moreover, the problem of counting finite objects on graphs (subgraphs, paths with a fixed length, etc) is important for mathematicians and it is a classical problem in discrete mathematics. The history about the question of counting contours of the same size containing a fixed unit cube on $\mathbb{Z}^{d}(d \geq 2)$ is the following: David Ruelle proved that there exist at most $3^{n}$ contours of size $n$ containing a fixed unit cube; Lebowitz and Mazel [123] proved that there are between $\left(C_{1} d\right)^{n / 2 d}$ and $\left(C_{2} d\right)^{64 n / d}$; and finally, differently from the previous approaches and using generating functions, Balister and Bollobás [12] improved these bounds showing that there are between $\left(C_{3} d\right)^{n / d}$ and $\left(C_{4} d\right)^{2 n / d}$ contours of size $n\left(C_{1}, C_{2}, C_{3}\right.$ and $C_{4}$ are constants).

In the last years, some attention was given to the Ising model on trees instead of $\mathbb{Z}^{d}$, and there is more than one definition of contour for trees and general graphs [10, 11, 123, 147, 149, 150, 151]. In our chapter we consider a definition proposed by Babson and Benjamini [11], where the contours are edge cuts which cut out exactly one finite component and they are minimal with this property. We will see that this definition on trees implies that the number of contours of size $n$ coincides with the number of external boundaries with $n$ vertices, a standard notion used by the combinatorics 
community. In the original paper they used the term cut sets as is usual for combinatorialists, the context was percolation theory, see also [14]. This definition was later considered in [10] in the study of bounds for the critical percolation probability $p_{c}$ in general graphs.

Our contribution is to clarify the connection between contours on trees and natural objects in graph theory. Inspired by Balister and Bollobás [12], we show that in the case of regular trees (and $d$-ary trees) we can calculate the exact number of contours of size $n$ containing a fixed vertex $x$. We also obtain a characterization for locally finite rooted trees with infinitely many contours of some fixed size $n$ involving the root. In particular, we prove that we have infinitely many contours of the same size if and only if the tree has an infinite independent path. Nonamenable trees are the trees in which the length of the independent paths is uniformly bounded. In particular, trees which contain an infinite independent path are amenable trees. On the other hand, for nonamenable graphs with bounded degree, (in particular, $d$-ary trees) one possibility for the proof of the phase transition in Ising models and for the study of ground states is to count the number of connected components of a fixed size containing a vertex, instead of counting the number of contours, see [82, 108].

This chapter is organized as follows: in Section 5.2 we give some basic definitions of graph theory, introduce the precise definition of a contour, and show the connection of these objects with external boundaries in graphs. In Section 5.3 we give explicit expressions for the number of contours of size $n$ in regular and $d$-ary trees. In addition, we show that the binary trees are extremal objects with respect to the number of contours of a fixed size. More precisely, if we fix $n$, the number of contours of size $n$ containing a fixed vertex is maximum in binary trees when we consider locally finite trees in which each vertex has at least two children. In Section 5.4 we give a geometric characterization of trees with infinitely many contours of the same size containing a fixed vertex. It turnes out that this is equivalent to the existence of what is called an infinite independent path in the tree.

\subsection{Definitions and Notations}

The graphs $G=(V, E)$ considered are always simple, undirected, connected, with countably infinite number of vertices. All the graphs are locally finite, in other words, with finite degree for each vertex of $V$. The degree of a vertex $x$ is the number of edges which are incident to $x$, denoted by $\mathrm{d}(x)$. A path $\gamma$ is an alternating sequence of vertices and edges $\gamma=\left(v_{0}, e_{1}, v_{1}, e_{2}, \ldots, e_{k}, v_{k}\right)$ where $e_{i}=v_{i-1} v_{i}=:\left\{v_{i-1}, v_{i}\right\}$ and all vertices are distinct, with the possible exception of $v_{0}, v_{k}$. The vertices $v_{1}, v_{2}, \ldots, v_{k-1}$ are called inner vertices of $\gamma$. An independent path $\gamma$ in a graph $G$ is a path where all inner vertices of $\gamma$ have degree two in $G$. When $v_{0}=v_{k}$ we say that the path $\gamma$ is a cycle. We say that a graph $G$ is a tree if it is connected and has no cycles.

Given a vertex $x$ and a subset of vertices $A \subset V$, let $\mathrm{d}_{G}(x, A)$ denote the number

$$
\mathrm{d}_{G}(x, A)=\min \{|\gamma| ; \gamma \text { is a path in } G \text { connecting } x \text { to a vertex of } A\},
$$

where for each path $\gamma$ in $G,|\gamma|$ denotes the number of edges of $\gamma$. $\operatorname{Thus~}_{G}(x, A)$ is the usual distance in the graph $G$ between $x$ and $A$. The set

$$
\partial_{\mathrm{v}}^{\text {ext }} A=\left\{x \in V \backslash A: \mathrm{d}_{G}(x, A)=1\right\}
$$

is the external boundary of $A$.

A rooted graph is a pair $(G, x)$, where $G$ is a graph and $x$ is a vertex of $G$. The vertex $x$ is called a root, and, for abuse of nomenclature, we say that $G$ is a rooted graph if there exists a root in $G$. For a rooted tree $(T, x)$, the generation $n$ is the set of vertices $y$ of $T$ with $\mathrm{d}(y, x)=n$. If $z$ is a vertex in $T$, a child of $z$ is a vertex $w$ incident to $z$ and $w$ belongs to the generation one after the generation where $z$ belongs to. In other words, the set of children of $z$ is $\left\{w \in \partial_{\mathrm{v}}^{\operatorname{ext}}(\{z\}): \mathrm{d}_{T}(x, w)=\mathrm{d}_{T}(x, z)+1\right\}$. A leaf on a tree is a vertex with no child, i.e., with degree one.

Let $G=(V, E)$ be a graph, we say that a graph $\tilde{G}$ is a minor of $G$, denoting by $\tilde{G} \preceq G$, when $\tilde{G}$ is obtained from $G$ after a sequence of the following operations: contracting some edges, deleting some edges and/or isolated vertices. We contract an edge $e=x y$ and obtain a graph that we denote 
by $G / e$ when we delete the edge $e$ from $E$, add to $E$ the collection of edges $\{a z ; x z \in E$ or $y z \in E\}$ where $a$ is a new vertex replacing the vertices $x$ and $y$, and remove all resulting parallel edges. Thus $V(G / e)=V(G) \backslash(\{x, y\}) \cup\{a\}$. We delete an edge $e=x y$ when we remove the edge from the graph but keep the vertices on it, after the process we obtain a new graph $G \backslash e=(V, E \backslash\{e\})$, for a finite collection of edges $C$ the procedure is the same, keeping the vertices and deleting the edges: $G \backslash C=(V, E \backslash C)$.

Definition 8. Given a graph $G=(V, E)$, a finite set $C \subset E$ is called a contour if $G \backslash C$ has exactly one finite connected component, and it is minimal with respect to this property. That is, for all edges e $\in C$ the graph $(V, E \backslash(C \backslash e))$ does not have a finite connected component.

If $C$ is a contour in $G$ then we denote by $G_{C}=\left(I_{C}, E_{C}\right)$ the unique finite connected component of $G \backslash C$.

This notion was originally defined by Babson and Benjamini in [11] where the authors used minimal cut set instead of contour. The definition was later used in [10] in the study of percolation problems on graphs.

Let $\mathcal{F}_{G}$ be the set of all contours of $G$. We denote by $\mathcal{F}_{G}^{n}$ the set of all contours of $G$ of size $n$; by $\mathcal{F}_{G}(x)\left(\mathcal{F}_{G}^{n}(x)\right)$ the set of all contours $C \in \mathcal{F}_{G}\left(C \in \mathcal{F}_{G}^{n}\right)$ such that $x \in I_{C}$.

Let $T_{d}$ be a rooted tree such that all vertices have $d$ children, i.e., the root has degree $d$ and the other vertices have degree $d+1$. The tree $T_{d}$ is called $d$-ary tree. A 2-tree is called binary tree. When all the vertices of a tree have the same degree $d$ we say that the tree is a $d$-regular tree.

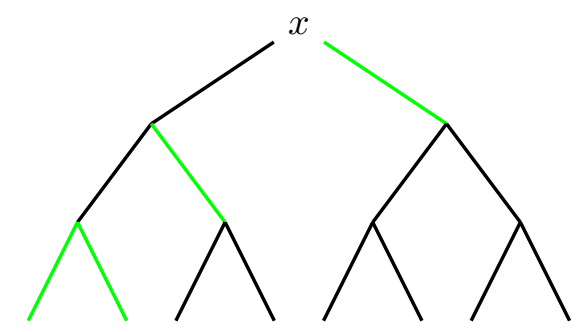

Figure 5.1: Example of a contour of size four in a binary tree $T_{2}$.

We conclude this section showing that in the case of trees there is a one-to-one correspondence between contours of size $\mathrm{n}$ in which the finite component contains the root and external boundaries of size $n$ of sets containing the root.

Proposition 11. Let $T=(V, E)$ be a rooted, locally finite and infinite tree. Let $x$ be the root and suppose that $T$ does not have any leaves. Let

$$
\mathcal{B}_{T}^{n}(x)=\left\{B \subset V: B \text { is finite, connected, } x \in B \text { and }\left|\partial_{\mathrm{v}}^{\operatorname{ext}}(B)\right|=n\right\}
$$

be the set of finite subtrees (induced by the vertices) of $T$ containing $x$ with external boundary of size $n$. Then there is a bijection between $\mathcal{B}_{T}^{n}(x)$ and $\mathcal{F}_{T}^{n}(x)$.

Proof. We define the function $f: \mathcal{F}_{T}^{n}(x) \rightarrow \mathcal{B}_{T}^{n}(x)$ in the following way: let $C$ be a contour in $\mathcal{F}_{T}^{n}(x)$. Remove all edges of $C$ from the tree $T$. By definition of contour, we get a finite connected component $B:=I_{C}$ containing $x$. Define $f(C)=B$. To show that $f$ is well defined, we shall prove that $\left|\partial_{\mathrm{v}}^{\operatorname{ext}}(B)\right|=n$.

By definition of contour, each edge in $C$ has one endpoint in $B$ and the other in $V \backslash B$. Let $V^{\text {ext }}(C)$ be the set of endpoints in $C \cap(V \backslash B)$. As $|C|=n$ and the graph is a tree, i.e., there is no cycle, we have $\left|V^{\mathrm{ext}}(C)\right|=n$. Clearly $V^{\mathrm{ext}}(C) \subseteq \partial_{\mathrm{v}}^{\text {ext }}(B)$. In fact, if $u \in V^{\text {ext }}(C)$, then there is a vertex $v \in B$ with $u v \in C$. Thus $\mathrm{d}(u, B)=1$.

Suppose that some element $u$ of $\partial_{\mathrm{v}}^{\text {ext }}(B)$ does not belong to $V^{\operatorname{ext}}(C)$, i.e., there is a vertex $v \in B$ incident to $u$ with $u v \notin C$. Since $C$ is a contour, then both vertices $u$ and $v$ should be in the same connected component. Since $v \in B$, then $u \in B$, a contradiction. Thus $f$ is well defined. 
To see that $f$ is surjective, note that for every $B \in \mathcal{B}_{T}^{n}(x)$ we can define $C_{B}$ be the set of edges where one of the endpoints belongs to $\partial_{\mathrm{v}}^{\operatorname{ext}}(B)$. Thus $\left|C_{B}\right|=n$. Let us show that $C=C_{B}$ is a contour. Note that the connected component containing the root $x$ after removing all the edges of $C$ on $T$ is the set $B$. Moreover, note also that $B$ is the unique finite connected component since the tree $T$ has no leaves. Suppose that there exists another finite connected component $B^{\prime}$ different of $B$, and consider $V\left(B^{\prime}\right)$ be the set of vertices of $B^{\prime}$. Since $C$ is finite and $T$ is a tree, one of the vertices in $V\left(B^{\prime}\right)$ is a leaf on $T$, contradicting the assumption that $T$ has no leaves. Thus, $C$ is a contour of size $n$.

The function $f$ is injective, since if $I_{C_{1}}=I_{C_{2}}$ are connected components containing $x$, then $C_{1}=C_{2}$.

\subsection{Contours on d-ary and regular trees}

The main technique in this section is the use of generating functions in the investigation of counting problems on trees; this approach produces very clean proofs. Classical references to the technique are the two books of Richard P. Stanley [163, 164].

The well known Catalan numbers $C_{n-1}=\frac{1}{n}\left(\begin{array}{c}2 n-2 \\ n-1\end{array}\right)$, with $n \in \mathbb{N}$, have a lot of interpretations in Combinatorics. In particular, see, e.g., [163, 164], these numbers count the number of contours in binary trees by Proposition 11. In fact, let $T_{2}$ be the binary tree with root $x$. For all $n \geq 2$, we have

$$
\left|\mathcal{F}_{T_{2}}^{n}(x)\right|=\frac{1}{n}\left(\begin{array}{c}
2 n-2 \\
n-1
\end{array}\right) .
$$

Here we present a proof where we calculate the exact number of contours in $d$-ary trees using generating functions, an alternative derivation can be found in [164]. Moreover, Proposition 12 allows us to conclude that for binary trees the number of contours of size $n$ containing the root is the $n$-th Catalan number. Let $\mathbb{R}((z))$ be the ring of formal series defined by

$$
\mathbb{R}((z))=\left\{\sum_{k \geq 0} a_{k} z^{k}: a_{k} \in \mathbb{R}\right\} .
$$

We define the operator $\left[z^{n}\right]$ which extracts the coefficient of $z^{n}$ in the series, that is,

$$
\left[z^{n}\right]\left(\sum_{k \geq 0} a_{k} z^{k}\right)=a_{n}
$$

We define $z \mathbb{R}((z))$ by

$$
z \mathbb{R}((z))=\left\{\sum_{k \geq 1} a_{k} z^{k}: a_{k} \in \mathbb{R}\right\} .
$$

The Lagrange Inversion Theorem states that we can compute exactly the coefficients of a series under certain conditions. The reader can find a proof of this theorem in $[69,164]$.

Theorem 31. Let $\phi \in \mathbb{R}((z))$ with $\phi(0) \neq 0$ and $f(z) \in z \mathbb{R}((z))$ defined by $f(z)=z \phi(f(z))$, then

$$
\left[z^{n}\right] f(z)=\left[z^{n-1}\right] \frac{1}{n} \phi(z)^{n} .
$$

Proposition 12. Let $d \geq 2, n \geq 1, T_{d}$ be a d-ary tree with root $x$. Then $\left|\mathcal{F}_{T_{d}}^{1}(x)\right|=0$ and, for $n \geq 2$,

$$
\left|\mathcal{F}_{T_{d}}^{n}(x)\right|=\left\{\begin{array}{ll}
\frac{1}{n}\left(\frac{d}{d-1}(n-1)\right. \\
\frac{1}{d-1}(n-1)
\end{array}\right), \quad \text { if } n \equiv 1 \quad(\bmod d-1),
$$


Proof. For each edge with endvertex $x$, we can either include this edge in the contour or not. If we do not include it, we carry the root $x$ to the other endvertex of this edge and apply again the same procedure.

Consider $f(X)=\sum_{n \geq 1} a_{n} X^{n}$ be the generating function in which the coefficients are $a_{n}=$ $\left|\mathcal{F}_{T_{d}}^{n}(x)\right|$ for all $n \geq 1$. We have

$$
f(X)=(X+f(X))^{d} .
$$

Consider $h(X)=X+f(X)$, we have $h(X)=X+h(X)^{d}$, so

$$
h(X)=X\left(1-h(X)^{d-1}\right)^{-1} .
$$

Applying Lagrange Inversion Theorem with $\phi(X)=\left(1-X^{d-1}\right)^{-1}$ we obtain

$$
\left[X^{n}\right] h(X)=\frac{1}{n}\left[X^{n-1}\right] \phi(X)^{n} .
$$

Now,

$$
\phi(X)^{n}=\left(1-X^{d-1}\right)^{-n}=\sum_{k \geq 0}\left(\begin{array}{c}
n+k-1 \\
k
\end{array}\right) X^{(d-1) k} .
$$

Thus, if $n-1=(d-1) k$ for some $k$, we have

$$
\left[X^{n}\right] h(X)=\frac{1}{n}\left(\begin{array}{c}
n+k-1 \\
k
\end{array}\right)=\frac{1}{n}\left(\begin{array}{c}
\frac{d}{d-1}(n-1) \\
\frac{1}{d-1}(n-1)
\end{array}\right),
$$

as we desired.

Remark: There is a geometric interpretation for the equation $h(X)=X+h(X)^{d}$. Consider $T_{d}$ be a $d$-ary tree with root $x$. Add an edge $e$ for which $x$ will be a leaf, and it will be an endpoint of $e$. Now we can either include the edge $e$ in the contour or not. If we do not include it, we carry the root $x$ to the other endvertex of this edge and apply the same procedure again.

Corollary 2. Let $d \geq 2, T_{d}$ be a d-ary tree with root $x$, and let $n \geq 1$ be such that $n \equiv 1(\bmod d-1)$, and $k=(n-1) /(d-1)$. We have

$$
\frac{1}{n} d^{k} \leq\left|\mathcal{F}_{T_{d}}^{n}(x)\right| \leq \frac{1}{n}(e d)^{k}
$$

Proof. This is a consequence of the following inequality. For all integers $0 \leq k \leq n$,

$$
\left(\frac{n}{k}\right)^{k} \leq\left(\begin{array}{l}
n \\
k
\end{array}\right) \leq\left(\frac{e n}{k}\right)^{k}
$$

Thus,

$$
\frac{1}{n} d^{k} \leq \frac{1}{n}\left(\begin{array}{c}
\frac{d}{d-1}(n-1) \\
\frac{1}{d-1}(n-1)
\end{array}\right) \leq \frac{1}{n}(e d)^{k},
$$

as we desired applying Proposition 12 .

Proposition 13. Let $d \geq 2, n \geq 1, T$ be $a(d+1)$-regular tree, and $x$ be a vertex of $T$. Then

$$
\left|\mathcal{F}_{T}^{n}(x)\right|=a_{n-1}+\sum_{k=1}^{n-1} a_{k} a_{n-k}
$$

where $a_{n}=\left|\mathcal{F}_{T_{d}}^{n}(x)\right|$. 
Proof. Let

$$
g(X)=\sum_{n \geq 1} b_{n} X^{n}
$$

be the generating function with coefficients $b_{n}=\left|\mathcal{F}_{T}^{n}(x)\right|$, and

$$
f(X)=\sum_{n \geq 1} a_{n} X^{n}
$$

be the generating function with coefficients $a_{n}=\left|\mathcal{F}_{T_{d}}^{n}(x)\right|$. Note that, since $f(X)=(X+f(X))^{d}$,

$$
\begin{aligned}
g(X) & =(X+f(X))^{d+1} \\
& =X f(X)+f(X)^{2} \\
& =X \sum_{n \geq 1} a_{n} X^{n}+\left(\sum_{n \geq 1} a_{n} X^{n}\right)^{2} \\
& =\sum_{n \geq 1} a_{n} X^{n+1}+\sum_{n \geq 2}\left(\sum_{k=1}^{n-1} a_{k} a_{n-k}\right) X^{n} \\
& =\sum_{n \geq 2}\left(a_{n-1}+\sum_{k=1}^{n-1} a_{k} a_{n-k}\right) X^{n} .
\end{aligned}
$$

Thus,

$$
b_{n}=a_{n-1}+\sum_{k=1}^{n-1} a_{k} a_{n-k}
$$

as we desired.

A natural question is to compare the number of contours between different infinite trees. We next show that the binary tree is extremal in the class of all locally finite trees in which every vertex has at least two children.

Theorem 32. Let $T$ be a locally finite and infinite rooted tree. Let $x$ be the root of $T$ and suppose that all vertices in $T$ have at least two children. Then, for all $n \geq 1$, we have $\left|\mathcal{F}_{T}^{n}(x)\right| \leq\left|\mathcal{F}_{T_{2}}^{n}(x)\right|$.

Proof. We will construct a binary labeled tree $T^{\prime}$ such that $T$ is a minor of $T^{\prime}$ as follows. We process the vertices of $T$ according to a Breadth-First-Search order, that is, we start from the root $x$, then process its neighbors, followed by their neighbors and so on. When we process a vertex $y$ of $T$ that has $s>2$ children, say $z_{1}, z_{2}, \ldots, z_{s}$, we replace $y$ by $s-1$ vertices $y_{1}, y_{2}, \ldots, y_{s-1}$. For each $i$ with $1 \leq i \leq s-2$, the children of $y_{i}$ are $y_{i+1}$ and $z_{i}$, and the children of $y_{s-1}$ are $z_{s-1}$ and $z_{s}$. When a vertex $y$ of $T$ has 2 children, we keep the vertex $y$ (See an example in Figures 5.2 and 5.3 for the first and the second iteration). We call the edges $y_{i} y_{i+1}$ by green edges (see Figures 5.2 and 5.3). Clearly $T^{\prime}$ is a binary tree. We call $x^{\prime}$ the root of $T^{\prime}$. We will show that there exists an injective map $f$ which takes each contour $C$ in $\mathcal{F}_{T}^{n}(x)$ and produces a contour $f(C)$ in $\mathcal{F}_{T^{\prime}}^{n}\left(x^{\prime}\right)$. In fact, for each edge of the form $y z_{i}$ in $C$, we associate the edge $y_{i} z_{i}$ in $T^{\prime}$ (for $y z_{s}$ take $y_{s-1} z_{s}$ ), and for $y$ with $s=2$ children we keep the edge $y z_{i}$. The collection of edges produced by this procedure is defined as $f(C)$.

We should prove that $f: \mathcal{F}_{T}^{n}(x) \rightarrow \mathcal{F}_{T^{\prime}}^{n}\left(x^{\prime}\right)$, in other words, that $f(C)$ belongs to $\mathcal{F}_{T^{\prime}}^{n}(x)$. By definition of the function $f$, there are no green edges in $f(C)$. Suppose by contradiction that $T^{\prime} \backslash f(C)$ has no finite connected component containing the root $x^{\prime}$. Thus there exists an infinite path $\gamma^{\prime}$ in $T^{\prime}$ starting at the root $x^{\prime}$ of $T^{\prime}$ without any green edges. When we contract all the green edges in $T^{\prime}$, in particular in $\gamma^{\prime}$, we obtain the original tree $T$ and a path $\gamma$ in $T$ starting in the root $x$. Since there are no green edges in the path $\gamma^{\prime}$, we have now an infinite path $\gamma$ in $T$ starting at the root $x$ with $E(\gamma) \cap C=\emptyset$, a contradiction. To see that $f(C)$ has the minimality property, let 
us show that for every edge $e^{\prime} \in f(C)$, the subgraph induced by the edges $E\left(T^{\prime}\right) \backslash\left(f(C) \backslash\left\{e^{\prime}\right\}\right)$ has an infinite component containing the root $x^{\prime}$. When we contract all the green edges and add the corresponding edge $e \in C$ (the edge associated to $e^{\prime}$ by $f$ ), since $C$ is a contour, there exists an infinite path $\alpha$ starting at the root $x$ in $T$ such that $e \in E(\alpha)$. We will construct, using the path $\alpha$, an infinite path $\alpha^{\prime}$ in $T^{\prime}$ starting at $x^{\prime}$ such that $e^{\prime} \in E\left(\alpha^{\prime}\right)$. Indeed, consider the process to construct the tree $T^{\prime}$ in the vertices of $\alpha$. Starting at the root $x$, for each edge $z y \in E(\alpha)$, where $y$ is a child of $z$, after processing $z$ there exists $1 \leq j \leq s-1$ such that $z_{j} y$ is an edge of $T^{\prime}$. Add $z_{j} y$ to $E\left(\alpha^{\prime}\right)$. If $j=1$ we add the edge $z_{1} y$ to $\alpha$, if $j>1$ we add the finite path starting in $z_{1}$ and ending in $z_{j}$, (which consists of green edges: $z_{1} z_{2}, z_{2} z_{3}, \ldots, z_{j-1} z_{j}$ ) and the edge $z_{j} y$ to $\alpha^{\prime}$. Since the path $\alpha$ is infinite and $e \in E(\alpha)$ we construct an infinite path $\alpha^{\prime}$, starting in $x^{\prime}$ such that $e^{\prime}$ belongs to $\alpha^{\prime}$. This shows that $f(C)$ is indeed a contour.

It is also easy to check that $f$ is injective and that $|C|=n$ implies $|f(C)|=n$.
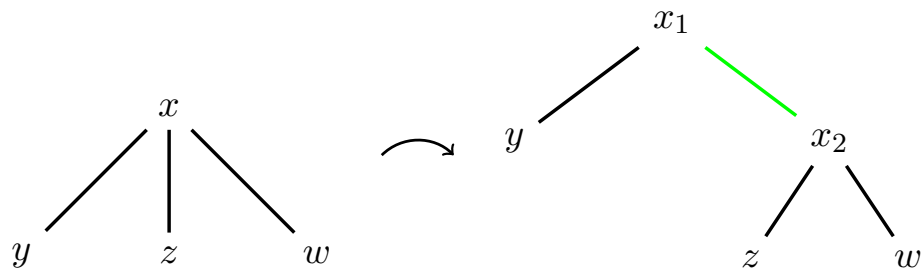

Figure 5.2: Example first iteration.

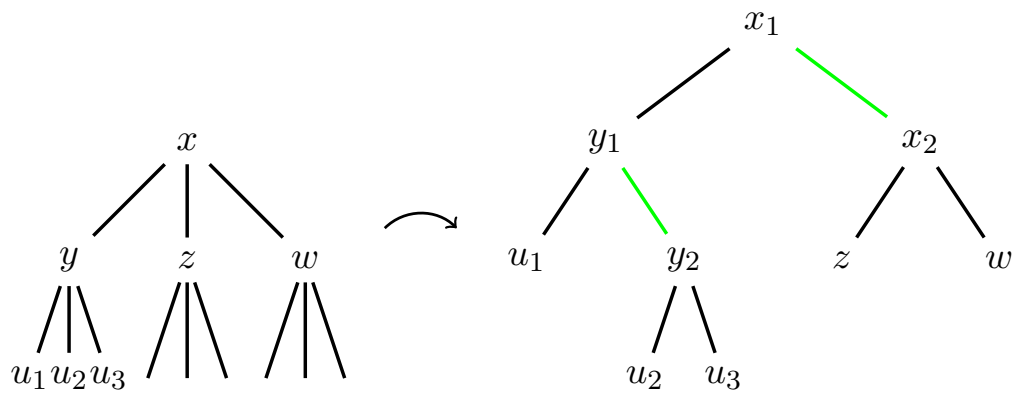

Figure 5.3: Part of the second iteration.

By Theorem 32 we have an estimate for trees in which each vertex has at least $r$ children, where $r \geq 2$. However, we have a better estimate for these trees. To prove this estimate we use the inequality below, which is a classical theorem in extremal combinatorics proved in [27], see also [8] and its references for several variants and extensions.

Theorem (Bollobás, 1965). Let $\left\{\left(A_{i}, B_{i}\right): i \in I\right\}$ be a finite collection of pairs of finite sets such that $A_{i} \cap B_{j}=\emptyset$ if and only if $i=j$. Then

$$
\sum_{i \in I}\left(\begin{array}{c}
\left|A_{i}\right|+\left|B_{i}\right| \\
\left|A_{i}\right|
\end{array}\right)^{-1} \leq 1 .
$$

In particular, if for all $i \in I$ we have $\left|A_{i}\right| \leq a$ and $\left|B_{i}\right| \leq b$, then

$$
|I| \leq\left(\begin{array}{c}
a+b \\
a
\end{array}\right)
$$


Theorem 33. Let $T$ be a rooted, locally finite and infinite tree with root $x$. Suppose that all vertices of $T$ have at least $r$ children, with $r \geq 2$. Then, for all $n \geq 1$,

$$
\left|\mathcal{F}_{T}^{n}(x)\right| \leq\left(\begin{array}{c}
n+\left\lfloor\frac{n-r}{r-1}\right\rfloor \\
\left\lfloor\frac{n-r}{r-1}\right\rfloor
\end{array}\right) .
$$

where $\lfloor x\rfloor=\max \{n \in \mathbb{Z}: n \leq x\}$.

Proof. Let $C \in \mathcal{F}_{T}^{n}(x)$ and let $I_{C}$ be the finite connected component when we remove $C$ from $T$. We will find an upper bound for the number of edges $\left|E\left(I_{C}\right)\right|$ in $I_{C}$. Let $B$ be the induced subgraph of $T$ on the union of $I_{C}$ and $C$. Note that $B=(V, E)$ is a rooted finite subtree of $T$ with $n$ leaves, and each vertex of $B$ that is not a leaf has at least $r$ children. Let $t$ be the number of vertices of $B$ and consider the number $k=t-n$. Note that $k$ is the number of vertices in $I_{C}$. Using the fact that all vertices of $T$ have at least $r$ children, we have

$$
2(t-1)=\sum_{v \in V} \mathrm{~d}(v) \geq(k-1)(r+1)+r+n .
$$

Thus, $k \leq(n-1) /(r-1)$.

Since $I_{C}$ is a tree, the number of edges in $I_{C}$ is $\left|E\left(I_{C}\right)\right|=k-1 \leq(n-r) /(r-1)$.

To finish the proof we need the following:

Fact: If $C_{1}$ and $C_{2}$ are two distinct contours of a vertex $x$ in $T$, each of size $n$, and if $I_{C_{1}}$ is the finite connected component when we remove $C_{1}$ from $T$, then $E\left(I_{C_{1}}\right) \cap C_{2} \neq \emptyset$.

Proof of the fact: Suppose, by contradiction, that there exist two contours $C_{1}$ and $C_{2}$ as above in $T$, each of size $n$, such that $E\left(I_{C_{1}}\right) \cap C_{2}=\emptyset$. Let $I_{C_{2}}$ be the finite connected component when we remove $C_{2}$ from $T$. Then $I_{C_{1}}$ is a subgraph of $I_{C_{2}}$ and $I_{C_{1}} \neq I_{C_{2}}$. Since $I_{C_{1}}$ and $I_{C_{2}}$ are subtrees of $T$, and as all vertices in $T$ have at least $r$ children, we have $\left|\partial_{e}\left(I_{C_{1}}\right)\right|<\left|\partial_{e}\left(I_{C_{2}}\right)\right|=n$, a contradiction. This proves the fact.

Finally, let us prove the desired result. Let $\left(C, E\left(I_{C}\right)\right)$ be a pair of a contour $C$ of size $n$, where $I_{C}$ is the finite connected component when we remove $C$ from $T$. We have $|C|=n$ and $\left|E\left(I_{C}\right)\right| \leq\lfloor(n-r) /(r-1)\rfloor$. The set $\left\{\left(C, E\left(I_{C}\right)\right): C \in \mathcal{F}_{T}^{n}(x)\right\}$ is finite, and $C_{1} \cap E\left(I_{C_{2}}\right)=\emptyset$ if and only if $C_{1}=C_{2}$. By the theorem above,

$$
\left|\mathcal{F}_{T}^{n}(x)\right| \leq\left(\begin{array}{c}
n+\left\lfloor\frac{n-r}{r-1}\right\rfloor \\
\left\lfloor\frac{n-r}{r-1}\right\rfloor
\end{array}\right)
$$

concluding the result.

It is sometimes desirable to consider contours whose edges cover a fixed vertex, see [147]. We obtain some bounds for this case as well.

Definition 9. Let $T$ be an infinite tree with root $x$. A rooted contour is a contour $C$ such that there exists an edge $l \in C$ incident with the root $x$.

We denote by $\mathcal{F}_{r, T}^{n}(x)$ the set of rooted contours $C$ on $T$ of size $n$. Clearly $\left|\mathcal{F}_{r, T}^{n}(x)\right| \leq\left|\mathcal{F}_{T}^{n}(x)\right|$. We can calculate exactly $\left|\mathcal{F}_{r, T}^{n}(x)\right|$ for $d$-ary trees and regular trees.

Proposition 14. Let $T_{d}$ be a d-ary tree with root $x$. Then, for all $n \geq d$ :

$$
\left|\mathcal{F}_{r, T_{d}}^{n}(x)\right|=a_{n}-\sum_{m_{1}+\ldots+m_{d}=n} a_{m_{1}} \ldots a_{m_{d}},
$$

where $a_{n}=\left|\mathcal{F}_{T_{d}}^{n}(x)\right|$. (Note that $\left|\mathcal{F}_{r, T_{d}}^{n}(x)\right|=0$ if $\left.n<d\right)$.

Proof. Let $f_{T_{d}}(X)=\sum_{n \geq 1} a_{n} X^{n}$ and $f(X)=\sum_{n \geq 1} c_{n} X^{n}$ be the generating functions with coefficients $a_{n}=\left|\mathcal{F}_{T_{d}}^{n}(x)\right|$ and $c_{n}=\left|\mathcal{F}_{r, T_{d}}^{n}(x)\right|$ respectively. For each edge incident with $x$ we can add 
it or not to the contour $C$. Repeating the same process as we did in Proposition 12, if we do not add an edge we carry the root to the other endpoint of this edge. By the same proposition we know $f_{T_{d}}(X)=\left(X+f_{T_{d}}(X)\right)^{d}$. Thus

$$
\begin{aligned}
f(X) & =\left(X+f_{T_{d}}(X)\right)^{d}-\left(f_{T_{d}}(X)\right)^{d} \\
& =f_{T_{d}}(X)-\left(f_{T_{d}}(X)\right)^{d} \\
& =\sum_{n \geq 1} a_{n} X^{n}-\left(\sum_{n \geq 1} a_{n} X^{n}\right)^{d} \\
& =\sum_{n \geq 1} a_{n} X^{n}-\sum_{n \geq d}\left(\sum_{m_{1}+\ldots+m_{d}=n} a_{m_{1}} \ldots a_{m_{d}}\right) X^{n} \\
& =\sum_{n \geq d}\left(a_{n}-\sum_{m_{1}+\ldots+m_{d}=n} a_{m_{1}} \ldots a_{m_{d}}\right) X^{n} .
\end{aligned}
$$

Thus, we have, for every $n \geq d$,

$$
c_{n}=a_{n}-\sum_{m_{1}+\ldots+m_{d}=n} a_{m_{1}} \ldots a_{m_{d}}
$$

as we desired.

Proposition 15. Let $T$ be a $(d+1)$-regular tree with root $x$. Then, for $n \geq d+1$,

$$
\left|\mathcal{F}_{r, T}^{n}(x)\right|=b_{n}-\sum_{m_{1}+\ldots+m_{d+1}=n} a_{m_{1}} \ldots a_{m_{d+1}},
$$

where $a_{n}=\left|\mathcal{F}_{T_{d}}^{n}(x)\right|$ and $b_{n}=\left|\mathcal{F}_{T}^{n}(x)\right|$.

Proof. Using a similar argument to the one used in the previous proof, let $f(X)=\sum_{n \geq 1} d_{n} X^{n}$ be the generating function with coefficients $d_{n}=\left|\mathcal{F}_{r, T}^{n}(x)\right|$ and let $g(X)=\sum_{n \geq 1} b_{n} X^{n}$ be the generating function from Proposition 13. Then

$$
\begin{aligned}
f(X) & =g(X)-\left(f_{T_{d}}(X)\right)^{d+1} \\
& =\sum_{n \geq 1} b_{n} X^{n}-\left(\sum_{n \geq 1} a_{n} X^{n}\right)^{d} \\
& =\sum_{n \geq d}\left(b_{n}-\sum_{m_{1}+\ldots+m_{d+1}=n} a_{m_{1}} \ldots a_{m_{d+1}}\right) X^{n} .
\end{aligned}
$$

Thus, for every $n \geq d$,

$$
d_{n}=b_{n}-\sum_{m_{1}+\ldots+m_{d+1}=n} a_{m_{1}} \ldots a_{m_{d+1}},
$$

as we desired.

\subsection{Infinitely many contours of size $n$}

Here we will characterize the rooted trees that have infinitely many contours for some size $n$ whose finite connected component contains the root. 
Notation 1. Let $G=(V, E)$ be a (possibly, infinite) graph. For each finite independent path $\gamma$ of $G$ linking two vertices $x$ and $y$, remove all the edges (and inner vertices) of $\gamma$ and add the edge $x y$. Denote by $\tilde{G}$ this new graph.

Note that $\tilde{G}$ is a minor of $G$, possibly with fewer edges.

In the next lemma and proposition the notation $\tilde{G}$ is used for this special case of minor.

Lemma 3. Let $T$ be a rooted, locally finite and infinite tree with root $x$. Suppose that $T$ has no leaves, and each independent path of $T$ has finite length. Then

$$
\left|\mathcal{F}_{T}^{n}(x)\right|<+\infty \text { if and only if }\left|\mathcal{F}_{\tilde{T}}^{n}(x)\right|<+\infty .
$$

Proof. Each contour $C=\left\{e_{1}, \ldots, e_{n}\right\}$ of $\tilde{T}$ is associated to a (unique) family of independent paths $\left\{\gamma_{1}, \ldots, \gamma_{n}\right\}$ of $T$. Then,

$$
\sum_{C \in \mathcal{F}_{\tilde{T}}^{n}(x)} \prod_{i=1}^{n}\left|\gamma_{i}\right|=\left|\mathcal{F}_{T}^{n}(x)\right| .
$$

Since the sum and the product are finite, we obtain $\left|\mathcal{F}_{T}^{n}(x)\right|<+\infty$. The converse is analogous.

Thus we obtain the following characterization.

Theorem 34. Let $T$ be a rooted, locally finite and infinite tree with a root $x$, and suppose that $T$ has no leaves. Then there exists $n \geq 1$ such that $\left|\mathcal{F}_{T}^{n}(x)\right|=+\infty$ if and only if $T$ has an infinite independent path.

Proof. Suppose that every independent path $T$ has finite length. Consider the minor $\tilde{T}$. We have that all vertices of $\tilde{T}$ have at least two children. By Theorem 32, we have $\left|\mathcal{F}_{\tilde{T}}^{n}(x)\right|<+\infty$. Therefore, by Lemma $3,\left|\mathcal{F}_{T}^{n}(x)\right|<+\infty$ for every $n \geq 1$.

For the converse, take an infinite independent path $\gamma$. Let $C$ be a contour of $T$ that contains an edge $e$ of $\gamma$. For all edge $e^{\prime}$ of $\gamma \backslash\{e\}$, we have that $C^{\prime}=(C \backslash\{e\}) \cup\left\{e^{\prime}\right\}$ is a contour of $T$ and $|C|=\left|C^{\prime}\right|$. Therefore, taking $n=|C|$ we obtain $\left|\mathcal{F}_{T}^{n}(x)\right|=+\infty$.

Proposition 16. Let $T$ be a rooted, locally finite and infinite tree with root $x$. Suppose that $T$ has no leaves and $T$ has an infinite independent path. Then there exists a sequence $\left(n_{i}\right)_{i \geq 1}$ such that $\left|\mathcal{F}_{T}^{n_{i}}(x)\right|=+\infty$ if and only if there is an infinite number of vertices in $T$ with degree at least three.

Proof. Suppose that there exists a finite number of vertices in $T$ with degree at least three. For every finite independent path in $T$, we do the same procedure to contract the path as in Notation 1. If an independent path in $T$ is infinite, we replace this independent path by a leaf. We denote this new tree by $T^{\prime}$. Since $T$ has an infinite independent path, $T^{\prime}$ has at least one leaf. Moreover, $T^{\prime}$ is a finite tree because $T$ has only a finite number of vertices with degree at least three. Let $B$ be a subtree of $T^{\prime}$ such that $x \in B$ and $B$ does not contain any leaf. Let $C$ be the set of external boundary edges of $B$. For each $C$ constructed in this way we obtain a family of contours of $T$ of the same size and any contour in $T$ comes from some external boundary edges for some such $B$. As we have a finite number of subtrees of $T^{\prime}$ that do not contain any leaves, there exists $n_{0} \geq 1 \mathrm{such}$ that for all $n \geq n_{0}$ we have $\left|\mathcal{F}_{T}^{n}(x)\right|=0$.

For the converse, suppose that there exists an infinite number of vertices in $T$ with degree at least three. Let $\mathcal{G}_{k}$ be the set of vertices in generation $k$, and $E_{k}$ be the set of edges $u v$ where $u \in \mathcal{G}_{k}$ and $v$ is a child of $u$. Note that $E_{k}$ is a contour. Since the number of vertices in $T$ with degree at least three is infinite, the number of edges in each $E_{k}$ in tending to infinity when we increase $k$. Let $\left(k_{i}\right)_{i}$ be an increasing sequence of natural numbers such that $n_{i}=\left|E_{k_{i}}\right|$ is also an increasing sequence. Let $\gamma$ be an infinite independent path. Note that there exists $i_{0}$ such that $E_{k_{i}}$ contains an edge $e_{i}$ of the infinite independent path $\gamma$ for all $i \geq i_{0}$. Then, since we can replace $e_{i}$ by any other edge of $\gamma$ and obtain a new contour of the same size $n_{i}$, we have infinitely many contours of size $n_{i}$. 


\subsection{Appendix: Other definitions of contours and the phase transi- tion}

Counting contours on graphs, such as the lattice $\mathbb{Z}^{d}$ and the Cayley trees, is useful to show phase transitions of statistical mechanical models by contour arguments. Let us present some notions of contours and they bounds. We will present Peierls' contours and bounds proved by Ruelle [154], Lebowitz and Mazel [123], and Balister and Bollobás [12], and also Rozikov's contours. Here, to avoid confusion with other definition of contours, the contours defined in Definition 8 will be called Babson-Benjamini contours.

\subsubsection{Peierls contours}

The first result on this way was by Peierls [135], who showed the phase transition of the ferromagnetic nearest-neighbor Ising model on the lattice $\mathbb{Z}^{d}$, with $d \geq 2$. Let us briefly explain the notion of Peierls' contour.

To be easier for visualization, let us consider the lattice $\mathbb{Z}^{2}$, and a finite connected set $\Lambda$ in $\mathbb{Z}^{2}$. Fix a configuration $\omega \in\{-1,1\}^{\mathbb{Z}^{2}}$, and consider the set of configurations inside the set $\Lambda$ with boundary condition $\omega$ by

$$
\Omega_{\Lambda}^{\omega}=\left\{\sigma \in\{-1,1\}^{\mathbb{Z}^{2}}: \sigma_{i}=\omega_{i} \text { for every } i \notin \Lambda\right\} .
$$

We write $\Omega_{\Lambda}^{+}$when the configuration of the boundary condition is $\omega_{i}=+1$ for every $i \in \mathbb{Z}^{2}$, and $\Omega_{\Lambda}^{-}$if $\omega_{i}=-1$ for every $i \in \mathbb{Z}^{2}$.

Now, to each vertex $i \in \mathbb{Z}^{d}$, consider the unit square centered at $i$ by

$$
\mathscr{S}_{i}=i+\left[-\frac{1}{2}, \frac{1}{2}\right]^{2}
$$

and, for two nearest-neighbors vertices $i$ and $j$, consider the set

$$
\pi_{i j}=\mathscr{S}_{i} \cap \mathscr{S}_{j}
$$

Note that $\pi_{i j}$ is an edge where the endpoints belong to the dual lattice $\mathbb{Z}_{*}^{2}$ defined by

$$
\mathbb{Z}_{*}^{2}=\mathbb{Z}^{2}+\left(\frac{1}{2}, \frac{1}{2}\right)=\left\{\left(i_{1}+\frac{1}{2}, i_{2}+\frac{1}{2}\right):\left(i_{1}, i_{2}\right) \in \mathbb{Z}^{2}\right\}
$$

Thus, let us call $\pi_{i j}$ by an dual-edge, and the edge $\{i, j\}$ by primal edge. Note that every primal edge intersects to an unique dual edge. Thus, for a given primal edge $e$ will denote by $e_{\perp}$ be the dual edge intersecting $e$. For a set of primal edges $C$, we denote by $C_{\perp}$ be the set of dual edges intersecting the set $C$.

For a fixed configuration $\sigma \in \Omega_{\Lambda}^{+}$, we are interested in the edges $\pi_{i j}$ where $i$ and $j$ are nearestneighbors with different spins. Consider the following set of dual-edges in $\Lambda$,

$$
\mathcal{D}(\sigma)=\bigcup_{\substack{i, j \in \mathbb{Z}^{2} \\\langle i, j\rangle \\ \sigma_{i} \neq \sigma_{j}}} \pi_{i j} .
$$

Note that $\mathcal{D}(\sigma)$ is a subgraph on $\mathbb{Z}_{*}^{2}$, and every vertex has even degree, 0,2 or 4 . For each connected component $B$ of $\mathcal{D}(\sigma)$, the interior of $B$, denoted by $\operatorname{Int}(B)$, is the set of vertices in $\mathbb{Z}^{2}$ of the bounded region when we consider $B$ as a curve in $\mathbb{R}^{2}$. We say $B$ is primitive if every vertex of $B$ has degree two. Figure 5.4 (found in [12]) shows examples of connected components.

For each connected component of $\mathcal{D}(\sigma)$ which is not primitive, we will apply the following deformation rule in Figure 5.5. 


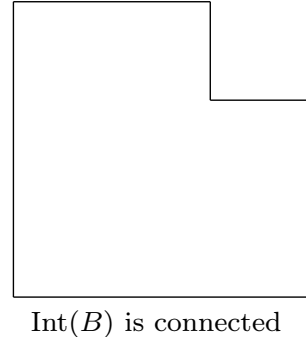
$B$ is primitive

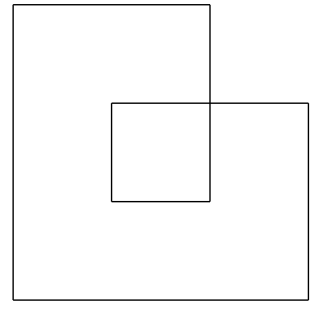

$\operatorname{Int}(B)$ is connected $B$ is not primitive

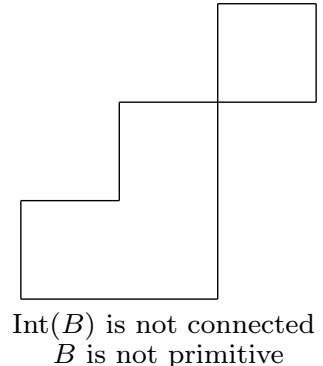

$B$ is not primitive

Figure 5.4: Examples of connected component in $\mathcal{D}(\sigma)$.
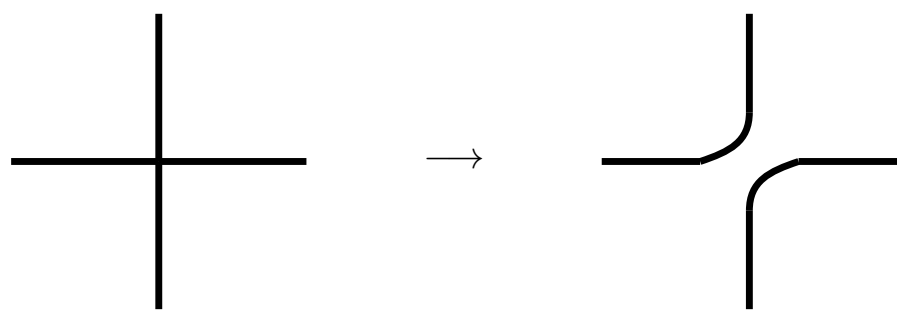

Figure 5.5: Deformation rule.

Thus, the set $\mathcal{D}(\sigma)$ is a set of disjoint simple cycles on the dual lattice,

$$
\mathcal{D}(\sigma)=\gamma_{1} \cup \ldots \cup \gamma_{k}
$$

these cycles $\gamma_{i}$ are called Peierls contours.

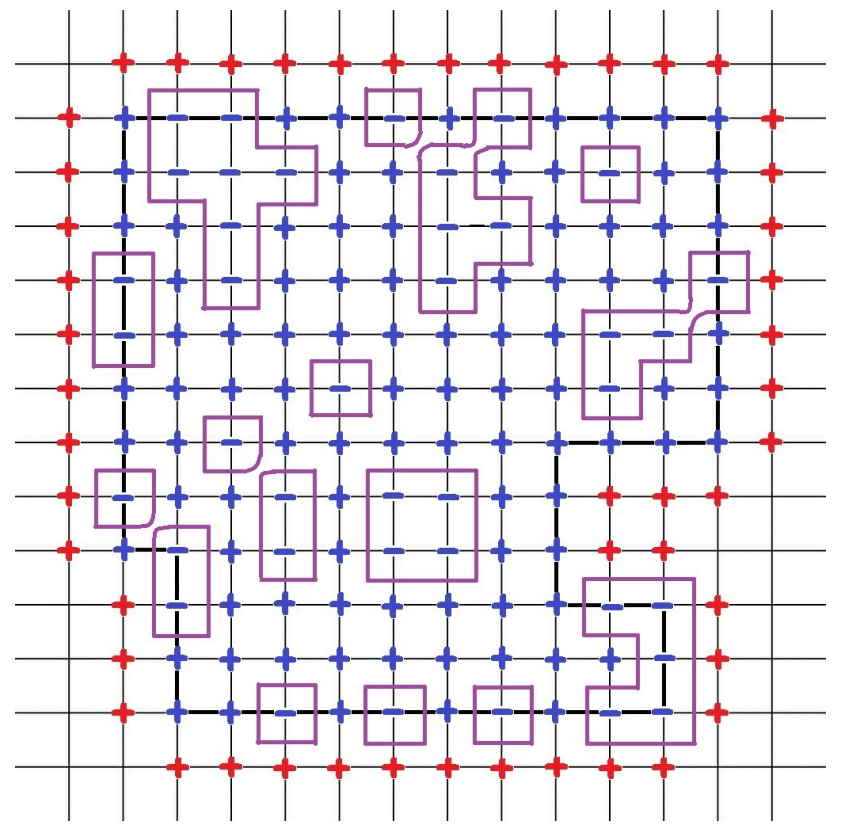

Figure 5.6: Set of contours on a finite set $\Lambda$.

Denote by $\Gamma(\sigma)=\left\{\gamma_{1}, \ldots, \gamma_{k}\right\}$ be the set of contours of $\sigma$. Denote by $|\gamma|$ be the number of dual edges in a contour $\gamma$, and by $\operatorname{Int}(\gamma)$ be the set of vertices in $\mathbb{Z}^{2}$ of the bounded region when we look $\gamma$ as a curve in $\mathbb{R}^{2}$.

We say that a Peierls contour $\gamma$ is primitive if, before the deformation rule, $\gamma$ is a primitive connected component.

Lemma 4. Let $C$ be a finite set of edges in $\mathbb{Z}^{2}$. The following are equivalent: 
1. $C$ is a Babson-Benjamini contour.

2. There exists a primitive Peierls contour $\gamma$ such that $C_{\perp}=\gamma$.

Proof. For a fixed Babson-Benjamini contour $C$ on $\mathbb{Z}^{2}$, let us show that there exists a configuration $\sigma \in\{-1,1\}^{\mathbb{Z}^{2}}$ such that $\Gamma(\sigma)=\left\{C_{\perp}\right\}$. Consider $G_{C}=\left(I_{C}, E_{C}\right)$ be the unique finite connected component of $\mathbb{Z}^{2} \backslash C$, and the following configuration $\sigma$.

$$
\sigma_{i}= \begin{cases}-1, & \text { if } i \in I_{C}, \\ +1, & \text { otherwise }\end{cases}
$$

Note that $u v \in C$ if, and only if, $\sigma_{u} \neq \sigma_{v}$. Thus,

$$
\mathcal{D}(\sigma)=\bigcup_{u v \in C} \pi_{u v}
$$

Suppose that there exists a connected component $B$ in $\mathcal{D}(\sigma)$ that is not primitive. If $\operatorname{Int}(B)$ is connected, then there exists an edge $e \in B$ such that $\mathbb{Z}^{2} \backslash(C \backslash e)$ contains a finite connected component, a contradiction. Otherwise, if $\operatorname{Int}(B)$ is not connected, then $\mathbb{Z}^{2} \backslash C$ contains two finite connected components, also a contradiction. Thus, every connected component in $\mathcal{D}(\sigma)$ is primitive.

Note that, if there are two disjoint primitive connected components $B_{1}$ and $B_{2}$ in $\mathcal{D}(\sigma)$, then we have two cases: If $\operatorname{Int}\left(B_{1}\right) \cup \operatorname{Int}\left(B_{2}\right)=\emptyset$, then $\operatorname{Int}\left(B_{1}\right)$ and $\operatorname{Int}\left(B_{2}\right)$ are two finite connected components on $\mathbb{Z}^{2} \backslash C$, contradicting the fact that $C$ is a Babson-Benjamini contour. Otherwise, if $\operatorname{Int}\left(B_{1}\right) \subset \operatorname{Int}\left(B_{2}\right)$, then for every dual edge $e_{\perp} \in B_{1}$, when we give back the primal edge $e$ intersecting $e_{\perp}$ in $\mathbb{Z}^{2} \backslash C$, we have that $\operatorname{Int}\left(B_{2}\right)$ is a finite connected component, contradicting again the fact that $C$ is a Babson-Benjamini contour. Therefore, $\mathcal{D}(\sigma)$ contains one connected component $\gamma$ which is primitive, and $\gamma=C_{\perp}$.

Now, suppose that $\gamma$ is a primitive Peierls contour, and consider the set of primal edges $C$ such that $C_{\perp}=\gamma$. Note that the induced graph of $\operatorname{Int}(\gamma)$ is connected and finite. Consider $\sigma \in\{-1,1\}^{\mathbb{Z}^{2}}$ be the configuration defined by

$$
\sigma_{i}= \begin{cases}-1, & \text { if } i \in \operatorname{Int}(\gamma) \\ +1, & \text { otherwise }\end{cases}
$$

Note that $\Gamma(\sigma)=\{\gamma\}$. Thus, $C$ is the set of edges $u v$ with $u \in \partial_{\mathrm{v}}^{\operatorname{ext}}(\operatorname{Int}(\gamma))$ and $v \in \operatorname{Int}(\gamma)$. Moreover, the unique finite connected component of $\mathbb{Z}^{2} \backslash C$ is $\operatorname{Int}(\gamma)$. Take any $e \in C$. Since $\gamma$ is primitive, the graph $\mathbb{Z}^{2} \backslash(C \backslash e)$ has no finite connected component. Thus, $C$ is a Babson-Benjamini contour.

Balister and Bollobás showed an estimate for $\left|\mathcal{F}_{\mathbb{Z}^{d}}^{k}(o)\right|$, where $o$ is the origin.

Theorem 35. There exist $C_{1}>0$ and $C_{2}>0$ such that, for every $d \geq 2$ and even $k \geq 4 d^{2}$,

$$
\left(C_{1} d\right)^{k / 2 d} \leq\left|\mathcal{F}_{\mathbb{Z}^{d}}^{k}(o)\right| \leq\left(C_{2} d\right)^{2 k / d} .
$$

The following lemma is sufficient to conclude the phase transition of the ferromagnetic nearestneighbor Ising model at low temperature, see [84, 135] for the proof.

Lemma 5. Consider a finite set $\Lambda$ on $\mathbb{Z}^{2}$. For every $\beta>0$ and any contour $\gamma$,

$$
\mu_{\Lambda, \beta}^{+}(\Gamma \ni \gamma) \leq e^{-2 \beta|\gamma|} .
$$

However, we need an estimate of the number of contours with a fixed size containing the origin, i.e., we need to find a good upper bound of the cardinality

$$
\rho(k)=\#\{\gamma: \operatorname{Int}(\gamma) \ni o,|\gamma|=k\},
$$


where $k \geq 1$. Here, good means an upper bound which is at most exponential order. Note that, by Lemma 4, we have

$$
\left|\mathcal{F}_{\mathbb{Z}^{d}}^{k}(o)\right| \leq \rho(k) .
$$

Let us now go back to the general lattice $\mathbb{Z}^{d}$ with $d \geq 2$. One easy upper bound is the following: For the first dual-edge there are $2 d$ options, and for the remaining $k-1$ dual-edges there are $2 d-1$ options for each one. Since every contour with size $k$ containing the origin should contain some vertex of the set $\left\{\left(i, i-\frac{1}{2}\right): 1 \leq i \leq k / 2\right\}$, we have

$$
\rho(k) \leq \frac{k}{2} \cdot 2 d \cdot(2 d-1)^{k} .
$$

Ruelle showed a better bound for (5.45). For $k$ sufficiently large and any $d \geq 2$,

$$
\rho(k) \leq k^{d} \cdot 3^{k} .
$$

The following theorem is due to Lebowitz and Mazel [123], who showed a better bound comparing with Ruelle.

Theorem 36. There exist $C_{3}>0$ and $C_{4}>0$ such that, for every $d \geq 2$ and sufficiently large even $k$,

$$
\left(C_{3} d\right)^{k / 2 d} \leq \rho(k) \leq\left(C_{4} d\right)^{64 k / d} .
$$

Theorem 36 implies the following upper bound for the critical inverse temperature of the Ising model. The "Peierls temperature" in high dimension gets further away from the true critical temperature, which approaches the mean-field temperature. Thus the $\log d$ is unavoidable, but indicates that we make larger mistakes in high $d$ (there also percolation of minuses in the plus phase easily happens; below the Peierls temperature this is excluded.)

Theorem 37. The polymer expansion constructed for the Ising model in terms of Peierls contours converges at inverse temperature $\beta$ for all $\beta \geq \frac{64}{d} \log d$. In particular, the critical inverse temperature is bounded by

$$
\beta_{c} \leq \frac{64}{d} \log d .
$$

So far, the best upper bound is due by Balister and Bollobás [12].

Theorem 38. There exist $C_{5}>0$ and $C_{6}>0$ such that, for every $d \geq 2$ and sufficiently large even $k$,

$$
\left(C_{5} d\right)^{k / d} \leq \rho(k) \leq\left(C_{6} d\right)^{2 k / d} .
$$

The proof is by using generating function and the geometry of the lattice $\mathbb{Z}^{d}$. Using Theorem 38 , and the result of Kotecký and Preiss [117] about the convergence of cluster expansion, it is possible to find the following upper bound for the critical temperature of the Ising model.

Theorem 39. The polymer expansion constructed for the Ising model in terms of Peierls contours converges at inverse temperature $\beta$ for all $\beta \geq \frac{2}{d} \log (11 d)$. In particular, the critical inverse temperature is bounded by

$$
\beta_{c} \leq \frac{2}{d} \log (11 d) .
$$

Indeed, there is a slightly better bound in [12],

$$
\beta_{c} \leq \frac{1}{d} \log \left(8 e^{21 / 8} d^{2}\right) \leq \frac{2}{d} \log (11 d) .
$$

However, Fernández and Procacci [67] obtained a new convergence criterion of cluster expansions that, comparing with Kotecký and Preiss, for some family of models it makes possible to find better estimates for the critical temperature, and this happens for Ising model. Let us present this result adapted for our purpose. 
Theorem 40. The polymer expansion constructed for the Ising model with coupling constant $J=1$, in terms of Peierls contours is convergent at inverse temperature $\beta$ if there exists a constant $\alpha>0$ such that, for any contour $\gamma$,

$$
\sum_{\gamma^{\prime}} e^{-(\beta-\alpha)\left|\gamma^{\prime}\right|} \leq e^{\alpha|\gamma|}-1
$$

where the sum is taken over all contours $\gamma^{\prime}$ that intersects $\gamma$.

Corollary 3. The polymer expansion constructed for the Ising model in terms of Peierls contours converges at inverse temperature $\beta$ for all

$$
\beta \geq \frac{1}{d} \log \left[8\left(\frac{1}{2 d}+1\right)^{d} e^{17 / 8} d^{2}\right] .
$$

In particular, the critical inverse temperature is bounded by

$$
\beta_{c} \leq \frac{1}{d} \log \left[8\left(\frac{1}{2 d}+1\right)^{d} e^{17 / 8} d^{2}\right] .
$$

The proof of Corollary 3 has the same proof of Theorem 12 in [12] applying Theorem 40. Note that this upper bound is asymptotically equal to $\frac{1}{d}\left(8 e^{21 / 8} d^{2}\right)$ for $d$ large enough. However, for small $d$ we have a better bound comparing with (5.52).

\subsubsection{Rozikov contours}

Rozikov also defined a notion of contours on trees in [147]. In this section, we are going to present the definition of Rozikov contours, and we will compare them with the set of rooted contours.

Let $T=(V, E)$ be an infinite, locally finite and rooted tree, and $x_{0} \in V$ be the root. Define the set $S=\left\{v_{1}, v_{2}, \ldots, v_{q}\right\}$, with $q \geq 2$. A configuration $\sigma$ on $V$ is defined as a function $x \in V \mapsto \sigma(x) \in S$.

Let $\Lambda \subset V$ be a finite set, $\Omega_{\Lambda}=S^{\Lambda}$ and $\sigma_{\Lambda} \in \Omega_{\Lambda}$ given configuration. For each $i$ we extend the configuration $\sigma_{\Lambda}$ inside $\Lambda$ to the entire tree by

$$
\sigma_{\Lambda}^{(i)}(t):= \begin{cases}\sigma_{\Lambda}(t), & \text { if } t \in \Lambda, \\ v_{i}, & \text { if } i \in \Lambda^{c} .\end{cases}
$$

Define $\Omega_{\Lambda}^{(i)}=\left\{\sigma_{\Lambda}^{(i)}: \sigma_{\Lambda} \in \Omega_{\Lambda}\right\}$ and the sets

$$
V_{n}:=\left\{x \in V: \mathrm{d}_{T}\left(x_{0}, x\right) \leq n\right\} \quad \text { and } \quad E_{n}:=\left\{x y \in E: x, y \in V_{n}\right\} .
$$

For a given configuration $\sigma_{\Lambda}^{(i)} \in \Omega_{\Lambda}^{(i)}$ and $j=1, \ldots, q$, denote

$$
V_{n}^{(j)}=V_{n}^{(j)}\left(\sigma_{\Lambda}^{(i)}\right):=\left\{t \in V_{n}: \sigma_{\Lambda}^{(i)}(t)=v_{j}\right\}
$$

Let $T^{n, j}=\left(V_{n}^{(j)}, E_{n}^{(j)}\right)$ be the tree, where the set of edges is defined by

$$
E_{n}^{(j)}=\left\{x y \in E: x, y \in V_{n}^{(j)}\right\} .
$$

It is clear that the tree $T^{n, j}$ contains a finite number $(=m)$ of maximal connected subtrees $T_{r}^{n, j}$, where $T_{r}^{n, j}=\left(V_{n, r}^{(j)}, E_{n, r}^{(j)}\right)$ for every $r=1, \ldots, m$, and $V_{n, r}^{(j)}$ is the set of vertices of $T_{r}^{n, j}$ and $E_{n, r}^{(j)}$ is the set of edges of $T_{r}^{n, j}$.

We define $i(x y)=\{x, y\}$ for every edge $x y \in E$. Two different edges $e_{1}, e_{2} \in E$ are called nearest neighboring edges if $\left|i\left(e_{1}\right) \cap i\left(e_{2}\right)\right|=1$, and we write $\left\langle e_{1}, e_{2}\right\rangle_{1}$.

For any connected component $K \subset T$, denote by $E(K)$ the set of edges of $K$, and

$$
b(K)=\left\{e \in E \backslash E(K): \text { there exists } e_{1} \in E(K) \text { such that }\left\langle e, e_{1}\right\rangle_{1}\right\} .
$$


The set $K=\operatorname{Int} b(K)$ is called interior of $b(K)$.

Definition 10. An edge $x y \in E_{n+1}$ is called a boundary edge of a configuration $\sigma_{V_{n}}^{(i)}$ if $\sigma_{V_{n}}^{(i)}(x) \neq$ $\sigma_{V_{n}}^{(i)}(y)$. The set of boundary edges of $\sigma_{V_{n}}^{(i)}$ is called boundary of $\sigma_{V_{n}}^{(i)}$, denoted by $\Gamma=\Gamma\left(\sigma_{V_{n}}^{(i)}\right)$.

A boundary $\Gamma$ can be decomposed as

$$
\Gamma=\bigcup_{i j \in Q_{q}} \Gamma_{i j}
$$

where $Q_{q}=\{i j: i<j$ and $i, j \in\{1, \ldots, q\}\}$ and $\Gamma_{i j}$ is the set of edges $x y$ with $\sigma(x)=v_{i}$ and $\sigma(y)=v_{j}$.

A subcontour of a boundary $\Gamma$ is a set

$$
\gamma=\gamma(n, j, r):=\left\{(x y, v(x), v(y)): x y \in b\left(T_{r}^{n, j}\right)\right\},
$$

where $j=1, \ldots, q$ and $r=1, \ldots, m$.

The set of edges of a subcontour $\gamma$ is denoted by $\operatorname{supp} \gamma$. We call mark, denoted by $v(\gamma) \in S$, the state of the spin of the configuration $\sigma_{V_{n}}^{(j)}$ in the interior of $\gamma$.

The collection of subcontours $\tau=\tau\left(\sigma_{V_{n}}^{(i)}\right):=\left\{\gamma_{r}: r=1, \ldots, m\right\}$ generated by the boundary $\Gamma$ of the configuration $\sigma_{V_{n}}^{(i)}$ satisfies the following properties:

(a) Every support of the subcontour $\gamma \in \tau$ lies inside the set $E_{n+1}$.

(b) For every two subcontours $\gamma_{1}, \gamma_{2} \in \tau$, their supports supp $\gamma_{1}$ and $\operatorname{supp} \gamma_{2}$ satisfy

$$
\left|\operatorname{supp} \gamma_{1} \cap \operatorname{supp} \gamma_{2}\right| \in\{0,1\} \text {. }
$$

The subcontours $\gamma_{1}, \gamma_{2} \in \tau$ are called adjacent if $\left|\operatorname{supp} \gamma_{1} \cap \operatorname{supp} \gamma_{2}\right|=1$.

(c) For any two adjacent subcontours $\gamma_{1}, \gamma_{2} \in \tau$ we have $v\left(\gamma_{1}\right) \neq v\left(\gamma_{2}\right)$.

A set of subcontours $\tilde{\tau} \subset \tau$ is connected if for any two subcontours $\gamma_{1}, \gamma_{2} \in \tilde{\tau}$ there exists a sequence of subcontours $\gamma_{1}=\tilde{\gamma}_{1}, \tilde{\gamma}_{2}, \ldots, \tilde{\gamma}_{l}=\gamma_{2}$ in $\tilde{\tau}$ such that, for each $i=1, \ldots, l-1$, the subcontours $\tilde{\gamma}_{i}$ and $\tilde{\gamma}_{i+1}$ are adjacent.

Definition 11. Any maximal connected (component) set of subcontours is called a Rozikov contour of boundary $\Gamma$.

Let $\Upsilon=\bigcup_{r \in I} \gamma_{r}$ be a contour of $\Gamma$. Denote:

(1) The interior of the Rozikov contour by Int $\Upsilon=\bigcup_{r \in I} \operatorname{Int} \gamma_{r}$.

(2) The support of the Rozikov contour by supp $\Upsilon=\bigcup_{r \in I} \operatorname{supp} \gamma_{r}$.

(3) The size of the Rozikov contour by $|\Upsilon|=|\operatorname{supp} \Upsilon|$.

For $n \geq 1$, we define $\mathcal{R F}_{T}^{n}\left(x_{0}\right)$ be the set of edges $\Upsilon$ with size $|\Upsilon|=n$ and $x_{0} \in \operatorname{Int} \Upsilon$ such that there exists a configuration $\sigma_{\Lambda}^{(i)}$ for some $i \in S$ and $\Lambda \Subset T$ such that $\Upsilon$ is a Rozikov contour of the configuration $\sigma_{\Lambda}^{(i)}$. We will show that there exists an injection from $\mathcal{F}_{r, T}^{n}\left(x_{0}\right)$ to $\mathcal{R F}_{T}^{n}\left(x_{0}\right)$.

Proposition 17. Let $S=\{-1,1\}$ and $T$ be a rooted, locally finite and infinite tree with root $x_{0}$, and $n \geq 1$. Then

$$
\left|\mathcal{F}_{r, T}^{n}\left(x_{0}\right)\right| \leq\left|\mathcal{R F}_{T}^{n}\left(x_{0}\right)\right|
$$


Proof. Let $C$ be a contour in $\mathcal{F}_{r, T}^{n}\left(x_{0}\right)$. Let $I_{C}$ be the finite connected component after removing the edges of $C$ on $T$. Define a configuration $\sigma \in\{-1,1\}^{V}$ by

$$
\sigma_{i}= \begin{cases}-1, & \text { if } i \in I_{C}, \\ 1, & \text { if } i \notin I_{C} .\end{cases}
$$

Note that $C$ is a Rozikov contours of the configuration $\sigma_{I_{C}}^{(1)}$.

\subsection{Final remarks and open questions}

The Peierls strategy to look for contours involving a vertex fails if $w$ in (5.1) depends only on the size of the contours when we have infinitely many contours of the same size. However, in [148] Rozikov studied an example of an Ising model type on $\mathbb{Z}$ where we have infinitely many contours of size 2 involving the vertex 0 . He adapted the Peierls argument to prove the phase transition for the model. In this case $w(C)$ must depend on the position of the contour $C$ in the graph, this is the usual situation when the Hamiltonian of the model is not translation invariant, see [21, 136].

Note that for nonamenable trees, every independent path is uniformly bounded by a constant, and thus the number of contours of size $n$ grows at maximum exponentially. For amenable trees, this does not happen anymore, since a tree with infinite independent paths is amenable. However, note that the trees containing an infinite number of finite independent paths, with length arbitrarily long, are also amenable. A question that we can raise is to find a characterization of the amenable trees that has the property that the number of contours of size $n$ grows at maximum exponentially for every $n$. 



\section{Chapter 6}

\section{Local Limits of Spatial Gibbs Random Graphs}

\subsection{Introduction}

In [132], the authors introduced and studied a class of random graphs which they called spatial Gibbs random graphs. These are random graphs embedded in an ambient space, which in [132], was a finite line segment. They are distributed according to a measure that penalizes the presence of edges whose extremities are distant (in terms of the ambient space geometry), but also penalizes graphs with large graph-theoretic diameter. Graphs sampled from this measure may thus be thought of as answering to a compromise between the conflicting requirements of using few long edges and having vertices close to each other in graph distance. The main result of [132] describes the typical aspect of these graphs depending on the various parameters that define them. Here, we continue the study of spatial Gibbs random graphs on line segments by considering their local convergence properties.

Let us explain the definition of spatial Gibbs random graphs and briefly present the results of [132]. Define the set of graphs on $\mathbb{Z}$ as

$$
\mathcal{G}=\{g=(V, E): V \subset \mathbb{Z} \text { and } g \text { is locally finite }\} .
$$

Given a graph $g=(V, E) \in \mathcal{G}$ and two vertices $x, y \in V$, the distance between $x$ and $y$ in $g$ is the smallest length over all paths in $g$ with endpoints $x$ and $y$. We denote this distance by $\mathrm{d}_{g}(x, y)$. Let $p \in[1, \infty]$; in case $g=(V, E) \in \mathcal{G}$ is finite, we define

$$
\mathcal{H}_{p}(g)= \begin{cases}\left(\frac{1}{\left(\begin{array}{c}
N \\
2
\end{array}\right)} \sum_{\substack{x, y \in V: \\
x<y}}\left(\mathrm{~d}_{g}(x, y)\right)^{p}\right)^{\frac{1}{p}} & \text { if } p \in[1, \infty) ; \\
\sup \left\{\mathrm{d}_{g}(x, y): x, y \in V\right\} & \text { if } p=\infty,\end{cases}
$$

that is, $\mathcal{H}_{\infty}(g)$ is the graph-theoretic diameter of $g$ and, if $p \in[1, \infty), \mathcal{H}_{p}(g)$ is a measure of typical distances in $g$.

For each $N \geq 1$ and $\gamma>0$, let $\mathbb{P}_{N, \gamma}$ be the probability measure on $\mathcal{G}$ supported on graphs $g=(V, E)$ with $V=[N]:=\{1, \ldots, N\}$ and $E \supset\{\{x, y\}: x, y \in V,|x-y|=1\}$, and so that the events

$$
\{\{x, y\} \in E\}_{x, y \in V,|x-y|>1}
$$

are independent, each having probability

$$
p_{\{x, y\}}=\exp \left\{-|x-y|^{\gamma}\right\} .
$$


We think of $\mathbb{P}_{N, \gamma}$ as a "reference measure" which we multiply by a Gibbs-type weight, thus obtaining a measure

$$
\mathbb{P}_{N, \gamma}^{b, p}(g)=\frac{1}{Z_{N, \gamma}^{b, p}} \cdot \exp \left\{-N^{b} \cdot \mathcal{H}_{p}(g)\right\} \cdot \mathbb{P}_{N, \gamma}(g), \quad g \in \mathcal{G},
$$

where $b \in \mathbb{R}, p \in[1, \infty]$ and $Z_{N, \gamma}^{b, p}$ is the normalization constant. In summary, this measure has four parameters: $N \in \mathbb{N}$ is the number of vertices of graphs over which it is supported, $\gamma>0$ controls the probabilities of the presence of edges in the reference measure, $p \in[1, \infty]$ determines the notion of typical distance that is used, and $b \in \mathbb{R}$ controls the sensitivity of the measure to the value of the typical distance. We denote by $G_{N}$ a random graph sampled from $\mathbb{P}_{N, \gamma}$ or $\mathbb{P}_{N, \gamma}^{b, p}$, depending on the context.

Under the reference measure $\mathbb{P}_{N, \gamma}$, the geometry of the random graph $G_{N}$ is not too different from that of the line segment on $[N]$. Indeed, using a simple analysis of "cutpoints" carried out in [132], it is not hard to show that, if $\varepsilon>0$ is small enough,

$$
\lim _{N \rightarrow \infty} \mathbb{P}_{N, \gamma}\left(\mathcal{H}_{p}\left(G_{N}\right)<\varepsilon N\right)=0 .
$$

This changes drastically by the introduction of the Gibbs weight (at least if the parameter $b$ is large enough). The main result of [132], reproduced as Theorem 41 below, is the convergence in probability of the random variable $N^{-1} \log \mathcal{H}_{p}\left(G_{N}\right)$ under $\mathbb{P}_{N, \gamma}^{b, p}$, when $\gamma, b, p$ are fixed and $N$ is taken to infinity. The limit is deterministic and given explicitly as a function of the parameters. Not all triples $(\gamma, b, p) \in(0, \infty) \times \mathbb{R} \times[1, \infty]$ are covered by the theorem: the case $\gamma=1$ is technically challenging and the proof of convergence for certain values of $(b, p)$ in that case is still missing. To identify this set of values, define for each $p \in[1, \infty]$ :

$$
\mathcal{E}_{p}= \begin{cases}\bigcup_{k=1}^{\infty}\left[\frac{k-1}{k}, \frac{k-1}{k}+\left(0 \vee \frac{2 p-(p-1) k}{k(k+1)(k+2 p)}\right)\right] & \text { if } p<\infty \\ {\left[0, \frac{1}{4}\right] \cup \bigcup_{k=2}^{\infty}\left\{\frac{k-1}{k}\right\}} & \text { if } p=\infty\end{cases}
$$

This set is plotted on Figure 6.1.

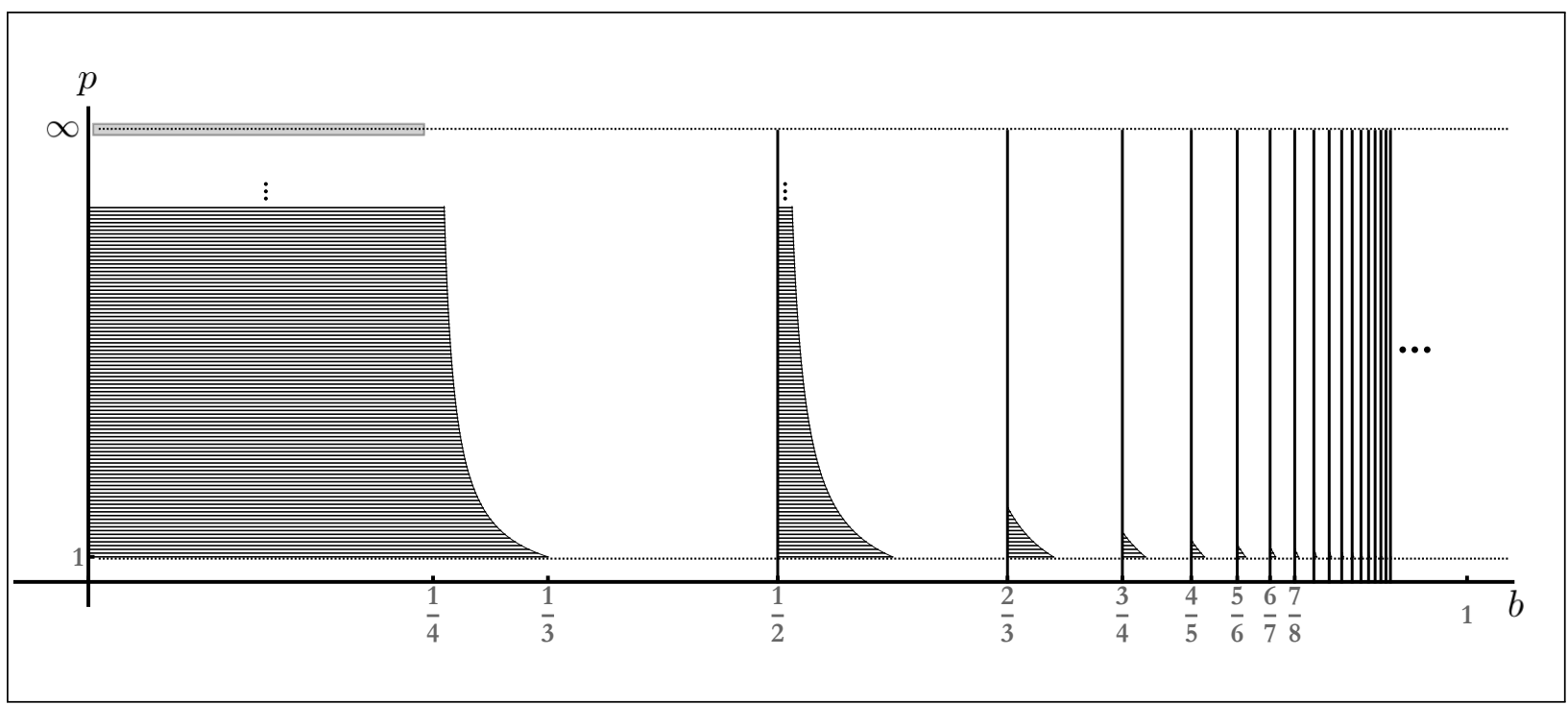

Figure 6.1: For each $p \in[1, \infty]$, the dark region represents the set $\mathcal{E}_{p}$, that is, the values of $b$ for which Theorem 41 does not cover the pair $(b, p)$ if $\gamma=1$. Note that, unless $p=1, \mathcal{E}_{p}$ only includes finitely many intervals and all numbers of the form $\frac{k-1}{k}, k \in \mathbb{N}$. 
Theorem 41 ([132]). In case

$$
\text { either } \gamma \neq 1, p \in[1, \infty], b \in \mathbb{R} \quad \text { or } \gamma=1, p \in[1, \infty], b \in \mathbb{R} \backslash \mathcal{E}_{p},
$$

for any $\varepsilon>0$,

$$
\mathbb{P}_{N, \gamma}^{b, p}\left(N^{\alpha^{*}-\varepsilon}<\mathcal{H}_{p}\left(G_{N}\right)<N^{\alpha^{*}+\varepsilon}\right) \stackrel{N \rightarrow \infty}{\longrightarrow} 1
$$

where

$$
\alpha^{*}=\alpha^{*}(\gamma, b)= \begin{cases}\left(\frac{1-b}{2-\gamma} \wedge 1\right) \vee 0 & \text { if } \gamma \in(0,1) ; \\ \left(\frac{\gamma-b}{\gamma} \wedge 1\right) \vee 0 & \text { if } \gamma>1 ; \\ \mathbb{1}_{(-\infty, 0)}(b)+\sum_{k=1}^{\infty} \frac{1}{k+1} \cdot \mathbb{1}_{\left[\frac{k-1}{k}, \frac{k}{k+1}\right)}(b) & \text { if } \gamma=1 .\end{cases}
$$

Note that the theorem identifies a "transition window" for the parameter $b$, given by the intervals $(-1+\gamma, 1),(0, \gamma)$ and $(0,1)$ respectively in the cases $\gamma \in(0,1), \gamma>1$ and $\gamma=1$. See Figure 6.2.

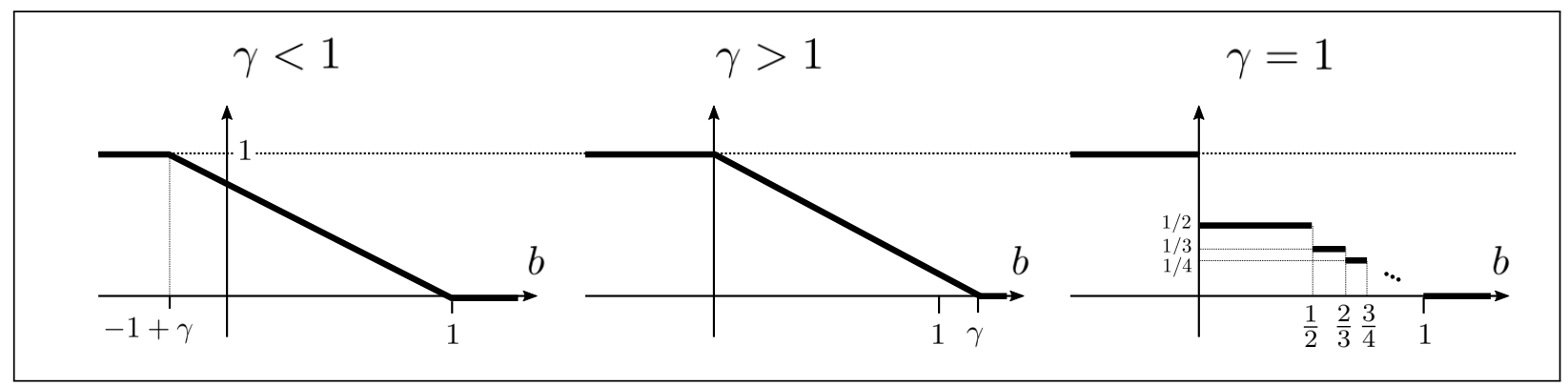

Figure 6.2: Plot of the function $b \mapsto \alpha^{*}(\gamma, b)$ of Theorem 41 for the three cases $\gamma \in(0,1), \gamma>1$ and $\gamma=1$.

In order to motivate our results, it is useful to give a brief exposition of what is involved in the proof of Theorem 41, carried out in [132]. Most of the work involves studying the reference measure; specifically, estimating $\mathbb{P}_{N, \gamma}\left(\mathcal{H}_{p}\left(G_{N}\right) \leq N^{\alpha}\right)$ as $N \rightarrow \infty$ for all values of $\alpha \in(0,1)$. Upper and lower bounds whose orders roughly match are obtained for these probabilities. To obtain a lower bound, the authors exhibit a graph $g^{\star}=g^{\star}(N, \gamma, \alpha)$ with $\mathcal{H}_{p}\left(g^{\star}\right)$ close to $N^{\alpha}$ and use the inequality

$$
\mathbb{P}_{N, \gamma}\left(\mathcal{H}_{p}\left(G_{N}\right) \leq N^{\alpha}\right) \geq \mathbb{P}_{N, \gamma}\left(g^{\star} \text { is a subgraph of } G_{N}\right) .
$$

The definition of $g^{\star}$ is completely different for the three cases $\gamma \in(0,1), \gamma>1$ and $\gamma=1$. In order to explain it, let us define, for $N \in \mathbb{N}$ and $\ell \in[N]$, the "layer" of edges

$$
\mathscr{E}_{N, \ell}=\{\{1,1+\ell\},\{1+\ell, 1+2 \ell\}, \ldots,\{1+(k-1) \ell, 1+k \ell\},\{1+k \ell, N\}\},
$$

where $k \geq 0$ is the integer satisfying $1+k \ell<N, 1+(k+1) \ell \geq N$. Then, $g^{\star}=([N], E)$ is defined as follows:

- in case $\gamma \in(0,1), E=\mathscr{E}_{N, 1} \cup \bigcup_{j=0}^{i} \mathscr{E}_{N, N 2^{-j}}$, where $i$ is the smallest integer with $N 2^{-i}<N^{1-\alpha}$;

- in case $\gamma>1, E=\bigcup_{j=0}^{i} \mathscr{E}_{N, 2^{j}}$, where $i$ is the smallest integer with $2^{i}>N^{\alpha}$;

- in case $\gamma=1, E=\mathscr{E}_{N, 1} \cup \bigcup_{j=1}^{i-1} \mathscr{E}_{N, N^{j / i}}$, where $i \geq 2$ is the integer such that $\alpha \in\left(\frac{1}{i}, \frac{1}{i-1}\right)$.

In all three cases, the layers which constitute $g^{\star}$ form hierarchical or fractal structures; they are added "from the top", "from the bottom" and "from the middle", respectively, when $\gamma<1, \gamma>1$ and $\gamma=1$. See Figures 6.3 and 6.4 for depictions of these graphs. The proof of the matching upper bound does not quite establish that the mentioned fractal structures are likely to be present in $G_{N}$. However, it does show that, in agreement with the definition of $g^{\star}$, the large-deviation event 
$\left\{\mathcal{H}_{p}\left(G_{N}\right) \leq N^{\alpha}\right\}$ with $\alpha \in(0,1)$ is most likely to occur due to a coordinated presence of long edges in case $\gamma \leq 1$ and a coordinated presence of short edges in case $\gamma>1$.

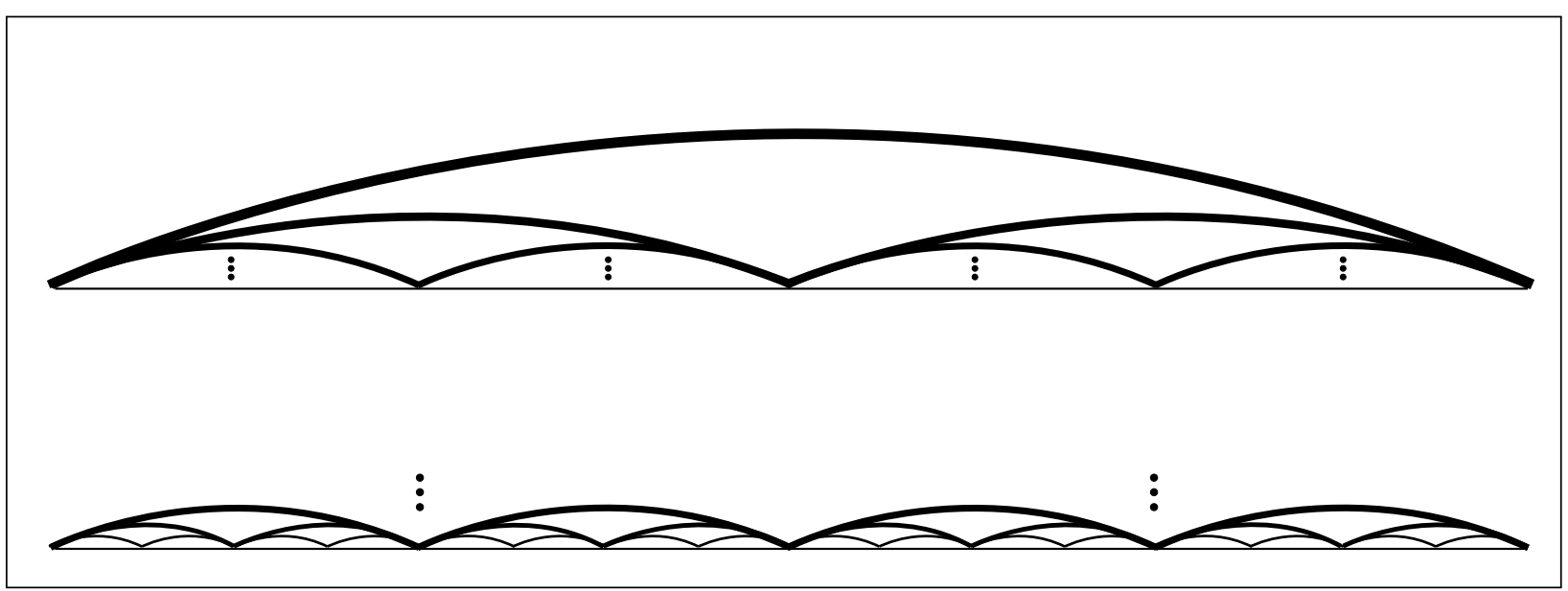

Figure 6.3: Hierarchical constructions that provide lower bounds for Theorem 41: case $\gamma<1$ (top) and $\gamma>1$ (bottom).

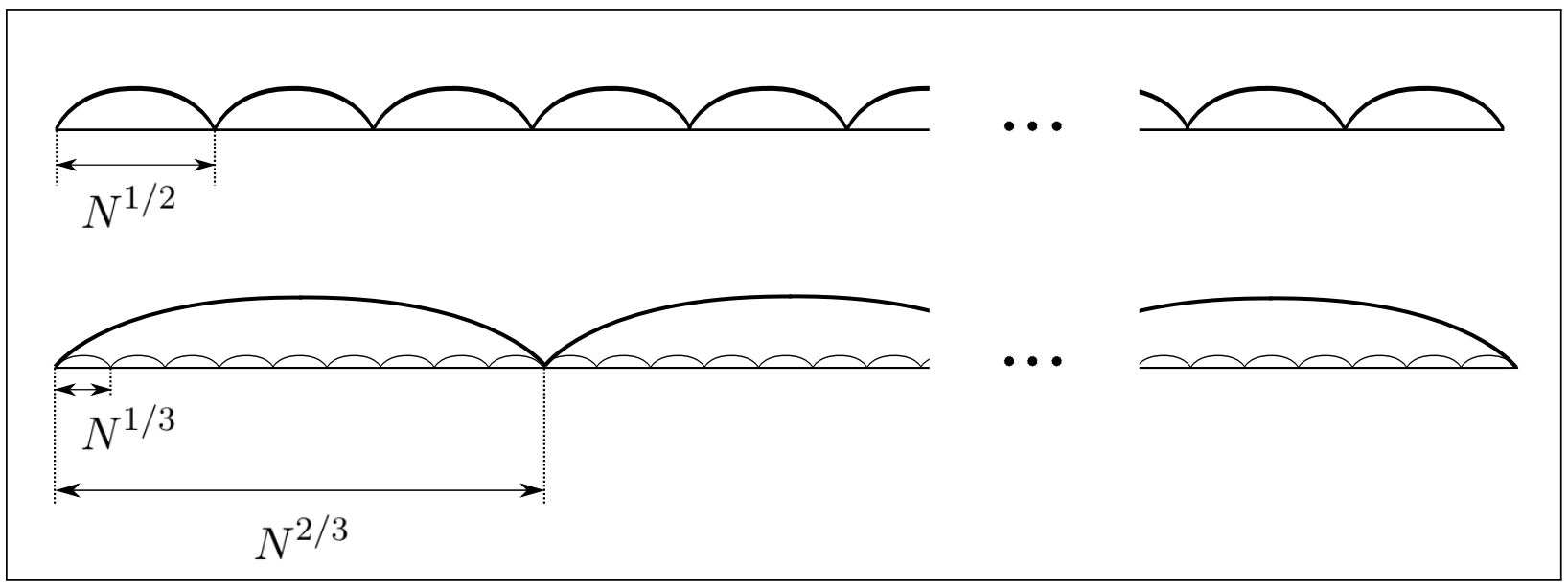

Figure 6.4: Hierarchical constructions that provide lower bounds for Theorem 41: case $\gamma=1$.

As already mentioned, in this paper we consider the local picture of the spatial Gibbs random graphs. The standard topology for local graph convergence is the one introduced by Benjamini and Schramm in [15]. This topology involves comparing rooted graphs by asking whether there are graph automorphisms between balls of different radii around the roots. Since here we consider graphs on $\mathbb{Z}$, the vertices of our graphs are labeled by natural numbers, so it makes sense to modify the Benjamini-Schramm convergence so as to demand that the automorphisms between balls respect the relative positions of the labels. This modification produces a finer topology (that is, if a sequence of rooted graphs converges in the sense to be given below, then it converges in the sense of [15]). Let us also mention that [13] also deals with an example of local convergence of rooted graphs endowed with labels or marks.

We now explain the ideas of the previous paragraph precisely. The set of rooted graphs on $\mathbb{Z}$ is defined by

$$
\mathcal{G}_{\bullet}=\{(g, o): g \in \mathcal{G}, o \text { is a vertex of } g\} .
$$

For $o, o^{\prime} \in \mathbb{Z}$, let $\varphi_{o, o^{\prime}}: \mathbb{Z} \rightarrow \mathbb{Z}$ be the translation

$$
\varphi_{o, o^{\prime}}(x)=x-o+o^{\prime}
$$


With abuse of notation, for a rooted graph $(g, o) \in \mathcal{G}$ • with $g=(V, E)$, and $o^{\prime} \in \mathbb{Z}$, we define $\varphi_{o, o^{\prime}}(g, o)=\left(\left(V_{\varphi}, E_{\varphi}\right), o^{\prime}\right) \in \mathcal{G}_{\bullet}$ as the rooted graph with

$$
V_{\varphi}=\varphi_{o, o^{\prime}}(V), \quad E_{\varphi}=\left\{\left\{\varphi_{o, o^{\prime}}(x), \varphi_{o, o^{\prime}}(y)\right\}:\{x, y\} \in E\right\} .
$$

For $g=(V, E) \in \mathcal{G}, o \in V, g^{\prime}=\left(V^{\prime}, E^{\prime}\right) \in \mathcal{G}$ and $o^{\prime} \in V^{\prime}$, we write $(g, o) \simeq\left(g^{\prime}, o^{\prime}\right)$ if $\varphi_{o, o^{\prime}}(g, o)=$ $\left(g^{\prime}, o^{\prime}\right)$.

Given $R>0$ and $(g, o) \in \mathcal{G}_{\bullet}$ with $g=(V, E)$, a ball with center $o$ and radius $R$ in $g$ is the rooted graph $B_{(g, o)}(R)=\left(\left(V_{B}, E_{B}\right), o\right) \in \mathcal{G}_{\bullet}$ of $g$ with

$$
V_{B}=\left\{x \in V: \mathrm{d}_{g}(o, x) \leq R\right\}, \quad E_{B}=\left\{\{x, y\} \in E: \mathrm{d}_{g}(o, x) \leq R \text { and } \mathrm{d}_{g}(o, y) \leq R\right\} .
$$

A sequence $\left(g_{n}, o_{n}\right) \in \mathcal{G}_{\bullet}$ is defined to converge to $(g, o) \in \mathcal{G}_{\bullet}$ in case

$$
\forall R \exists n_{0}: n \geq n_{0} \Longrightarrow B_{\left(g_{n}, o_{n}\right)}(R) \simeq B_{(g, o)}(R) .
$$

The associated notion of convergence in distribution is as follows. Given a sequence of random rooted graphs $\left(G_{n}, \mathcal{O}_{n}\right)$ defined under the probability measure $\mu_{n}$ and a random rooted graph $(G, \mathcal{O})$ defined under the probability measure $\mu$, the sequence $\left(G_{n}, \mathcal{O}_{n}\right)$ converges in distribution to $(G, \mathcal{O})$ if for all $R>0$, and for any deterministic rooted graph $(g, o) \in \mathcal{G}_{\bullet}$, we have

$$
\lim _{n \rightarrow \infty} \mu_{n}\left(B_{\left(G_{n}, \mathcal{O}_{n}\right)}(R) \simeq(g, o)\right)=\mu\left(B_{(G, \mathcal{O})}(R) \simeq(g, o)\right) .
$$

Let us give an example that will be useful for the statement of our main result. Let $\mathbb{P}_{\gamma}$ be the measure on $\mathcal{G}$ supported on graphs $g=(V, E)$ with $V=\mathbb{Z}$ and $E \supset\{\{x, y\}: x, y \in V,|x-y|=1\}$, and so that the events as in (6.3) are independent, with probabilities as in (6.4). If $G_{N}$ is sampled from $\mathbb{P}_{N, \gamma}, G$ is sampled from $\mathbb{P}_{\gamma}$ and $a_{N}$ is a sequence with $1 \ll a_{N} \ll N$, then it is easy to see that $\left(G_{N}, a_{N}\right)$ converges in distribution to $(G, 0)$.

Note that this convergence is stronger than the well-known local convergence of graphs defined by Benjamini and Schramm in [15]. In fact, the definition of the local convergence by Benjamini and Schramm is the following: Let $(G, o)$ and $\left(G_{j}, o_{j}\right)_{j \geq 1}$ be random connected rooted graphs. We say that $(G, o)$ is the Benjamini-Schramm limit of $\left(G_{j}, o_{j}\right)$ as $j \rightarrow \infty$ if for every $r>0$ and for every finite rooted graph $\left(H, o^{\prime}\right)$, the probability that $\left(H, o^{\prime}\right)$ is isomorphic to $\left(B_{\left\{G_{j}, o_{j}\right\}}(r), o_{j}\right)$ converges to the probability that $\left(H, o^{\prime}\right)$ is isomorphic to $\left(B_{\{G, o\}}(r), o\right)$. Thus, while in the Benjamini-Schramm convergence needs the existence of some isomorphisms, the isomorphism in our convergence should be $\varphi_{o, o^{\prime}}$.

We now state our main result.

Theorem 42. Assume $p \in[1, \infty]$ and either of the following conditions hold:

$$
[\gamma \in(0,1), b \in(-\infty, 1)], \quad\left[\gamma=1, b \in(-\infty, 1) \backslash \mathcal{E}_{p}\right], \quad \text { or } \quad[\gamma>1, b \in(-\infty, 0)] .
$$

Let $\mathcal{U}_{N}$ be the uniform measure on $\{1, \ldots, N\}$. Then, $\left(G_{N}, \mathcal{O}_{N}\right)$ sampled from $\mathbb{P}_{N, \gamma}^{b, p} \otimes \mathcal{U}_{N}$ converges in distribution to $(G, \mathcal{O})$ sampled from $\mathbb{P}_{\gamma} \otimes \delta_{\{0\}}$.

Intuitively, this result states that, if one of the three conditions holds, then graphs sampled from $\mathbb{P}_{N, \gamma}$ and $\mathbb{P}_{N, \gamma}^{b, p}$ are indistinguishable from the point of view of local convergence; in other words, the presence of the Gibbs weight $\exp \left\{-N^{b} \cdot \mathcal{H}_{p}(g)\right\}$ has no impact on the local picture. Note that, for the regimes $\gamma \in(0,1)$ and $\gamma=1$, this is compatible with the heuristic explanation we have provided above for the proof of Theorem 41: in both cases, graphs are most likely to achieve a small diameter by deviating from the reference measure in their long-edge configuration. In the remaining case $[\gamma>1, b<0]$, the idea is that the Gibbs weight is not sufficiently large to cause the random graph to deviate from its local aspect under the reference measure.

Taking this into account, it is not surprising that the study of local convergence is harder for $\gamma>1, b \geq 0$ : in that case, short edges do most of the job of reducing the diameter of the graph, so 
the local picture should be affected by the Gibbs weight. If there is a limiting distribution at all, it would likely differ from $\mathbb{P}_{\gamma}$. Our results in this direction are more modest: we show that for a certain subset of the relevant parameters, there is no convergence in distribution.

Proposition 18. For $L>0$, let $\mathcal{L}_{L}$ be the set of graphs $g=(V, E) \in \mathcal{G}$ with the property that, if $x, y \in V$ with $0<|x-y| \leq L$, then $\{x, y\} \in E$. For any $L>0, \gamma>1, p<\infty$ and $b>p+1$, then

$$
\mathbb{P}_{N, \gamma}^{b, p}\left(G_{N} \in \mathcal{L}_{L}\right) \stackrel{N \rightarrow \infty}{\longrightarrow} 1 \text {. }
$$

In particular (since $\mathcal{G}$ consist only of locally finite graphs), the sequence $\left(G_{N}, \mathcal{O}_{N}\right)$ sampled from $\mathbb{P}_{N, \gamma}^{b, p} \otimes \mathcal{U}_{N}$ does not converge in distribution.

\subsection{Proof of main result}

\subsubsection{Truncated balls and proof of Theorem 42}

In order to prove Theorem 42, it is enough to prove the following statement.

The natural approach to prove (6.14) is to first show that, under the reference measure $\mathbb{P}_{N, \gamma}$, the graph $G_{N}$ has certain desirable properties with high probability, and then to use this to draw the desired conclusion about the weighted measure $\mathbb{P}_{N, \gamma}^{b, p}$. This approach is indeed natural because of the independence properties of the reference measure, which make it easier to study than the weighted measure. However, note that for $i, j \in[N]$, events of the form $\left\{B_{\left(G_{n}, i\right)}(k) \simeq(g, o)\right\}$ and $\left\{B_{\left(G_{n}, j\right)}(k) \simeq(g, o)\right\}$ are not independent even if $|i-j|$ is large, as both events could be influenced by the presence of long edges with extremities in the vicinities of $i$ and $j$. To deal with this problem, we will introduce truncated balls below.

Given an edge $e=\{i, j\}$ of a graph on $\mathbb{Z}$, we define the length of $e$ as $|e|:=|i-j|$. Given the rooted graph $(g, o)$ and $k, L \in \mathbb{N}$, we define the truncated ball $B_{(g, o)}^{L}(k)$ as follows. Let $g^{\prime}$ be the graph obtained from $g$ by removing all edges with length larger than $L$; then, we let $B_{(g, o)}^{L}(k)=B_{\left(g^{\prime}, o\right)}(k)$.

The essential ingredients in our proof of (6.14) are given in the following result.

Proposition 19. Fix $\gamma, p, b$ as in (6.10).

1. For any $k, L>0,(g, o) \in \mathcal{G}_{\bullet}$ and $\varepsilon>0$,

$$
\mathbb{P}_{N, \gamma}^{b, p}\left(\left|\frac{\#\left\{i \in[N]: B_{\left(G_{N}, i\right)}^{L}(k) \simeq(g, o)\right\}}{N}-\mu_{\gamma}^{L}(k,(g, o))\right|>\varepsilon\right) \stackrel{N \rightarrow \infty}{\longrightarrow} 0,
$$

where

$$
\mu_{\gamma}^{L}(k,(g, o))=\mathbb{P}_{\gamma}\left(B_{(G, 0)}^{L}(k) \simeq(g, o)\right) .
$$

2. For any $\varepsilon>0$ there exists $L>0$ such that

$$
\mathbb{P}_{N, \gamma}^{b, p}\left(G_{N} \text { has more than } \varepsilon N \text { edges with length larger than } L\right) \stackrel{N \rightarrow \infty}{\longrightarrow} 0 .
$$

Let us show how Proposition 19 gives the proof of Theorem 42; the proof of Proposition 19 will be given afterwards.

Proof of Theorem 42. Fix $\gamma, p, b$ as in (6.10). Also fix $k \in \mathbb{N},(g, o) \in \mathcal{G} \bullet$ and $\varepsilon>0$. First, let us show that

$$
\mathbb{P}_{N, \gamma}^{b, p}\left(\left|\frac{\#\left\{i \in[N]: B_{\left(G_{N}, i\right)}(k) \simeq(g, o)\right\}}{N}-\mu_{\gamma}(k,(g, o))\right|>\varepsilon\right) \stackrel{N \rightarrow \infty}{\longrightarrow} 0,
$$

where

$$
\mu_{\gamma}(k,(g, o)):=\mathbb{P}_{\gamma}\left(B_{(G, 0)}(k) \simeq(g, o)\right)
$$


For each $L>0$, let $\mu_{\gamma}^{L}(k,(g, o))$ be as in (6.12). Using the fact that $\mathbb{P}_{\gamma}$ is supported on locally finite graphs, it is easy to verify that

$$
\lim _{L \rightarrow \infty} \mu_{\gamma}^{L}(k,(g, o))=\mu_{\gamma}(k,(g, o)) .
$$

We can thus choose $L$ large enough that

$$
\left|\mu_{\gamma}^{L}(k,(g, o))-\mu_{\gamma}(k,(g, o))\right|<\varepsilon / 2 .
$$

Then, by the triangle inequality,

$$
\begin{aligned}
\varepsilon & <\left|\frac{\#\left\{i: B_{\left(G_{N}, i\right)}^{L}(k) \simeq(g, o)\right\}}{N}-\mu_{\gamma}(k,(g, o))\right| \\
& \leq\left|\frac{\#\left\{i: B_{\left(G_{N}, i\right)}(k) \simeq(g, o)\right\}}{N}-\frac{\#\left\{i: B_{\left(G_{N}, i\right)}^{L}(k) \simeq(g, o)\right\}}{N}\right| \\
& +\left|\frac{\#\left\{i: B_{\left(G_{N}, i\right)}^{L}(k) \simeq(g, o)\right\}}{N}-\mu_{\gamma}^{L}(k,(g, o))\right| \\
& +\left|\mu_{\gamma}^{L}(k,(g, o))-\mu_{\gamma}(k,(g, o))\right|
\end{aligned}
$$

implying that

$$
\mid \begin{aligned}
& \left|\frac{\#\left\{i: B_{\left(G_{N}, i\right)}^{L}(k) \simeq(g, o)\right\}}{N}-\mu_{\gamma}^{L}(k,(g, o))\right|>\frac{\varepsilon}{4} ; \text { or } \\
& \left|\frac{\#\left\{i: B_{\left(G_{N}, i\right)}(k) \simeq(g, o)\right\}}{N}-\frac{\#\left\{i: B_{\left(G_{N}, i\right)}^{L}(k) \simeq(g, o)\right\}}{N}\right|>\frac{\varepsilon}{4} .
\end{aligned}
$$

It is then sufficient to prove that

$$
\mathbb{P}_{N, \gamma}^{b, p}\left(\left|\frac{\#\left\{i: B_{\left(G_{N}, i\right)}^{L}(k) \simeq(g, o)\right\}}{N}-\mu_{\gamma}^{L}(k,(g, o))\right|>\frac{\varepsilon}{4}\right) \stackrel{N \rightarrow \infty}{\longrightarrow} 0
$$

and

$$
\mathbb{P}_{N, \gamma}^{b, p}\left(\left|\frac{\#\left\{i: B_{\left(G_{N}, i\right)}(k) \simeq(g, o)\right\}}{N}-\frac{\#\left\{i: B_{\left(G_{N}, i\right)}^{L}(k) \simeq(g, o)\right\}}{N}\right|>\frac{\varepsilon}{4}\right) \stackrel{N \rightarrow \infty}{\longrightarrow} 0 .
$$

The convergence (6.16) is given directly by (6.11). For (6.17), first observe that, if $L$ is large (regardless of $N)$,

$$
\left\{i \in[N]: B_{\left(G_{N}, i\right)}(k) \simeq(g, o)\right\} \subseteq\left\{i \in[N]: B_{\left(G_{N}, i\right)}^{L}(k) \simeq(g, o)\right\} .
$$

Moreover, if $i_{0} \in[N]$ belongs to the set on the right-hand side but not to the set on the left-hand side, then there exists a vertex $x$ of $g$ such that $i_{0}+x-o$ is an extremity of some edge $e$ of $G_{N}$ with $|e|>L$. Using these observations, we obtain

$$
\begin{aligned}
& \mid \#\left\{i \in[N]: B_{\left(G_{N}, i\right)}(k)\right.\simeq(g, o)\}-\#\left\{i \in[N]: B_{\left(G_{N}, i\right)}^{L}(k) \simeq(g, o)\right\} \mid \\
& \leq 2 \cdot \#\{\text { vertices of } g\} \cdot \#\left\{\text { edges of } G_{N} \text { with length larger than } L\right\} .
\end{aligned}
$$

Hence, (6.17) follows from (6.13), concluding (6.14). 
Let us consider the event

$$
A=A(\gamma,(g, o), \varepsilon, k, N)=\left\{\left|\frac{\#\left\{i: B_{\left(G_{N}, i\right)}(k) \simeq(g, o)\right\}}{N}-\mu_{\gamma}(k,(g, o))\right|>\varepsilon\right\} .
$$

For a fixed $\delta>0$ and $N$ sufficiently large such that

$$
\mathbb{P}_{N, \gamma}^{b, p}(A(\gamma,(g, o), \delta / 3, k, N))<\delta / 3,
$$

we have

$$
\begin{aligned}
\mathbb{P}_{N, \gamma}^{b, p} \otimes \mathcal{U}_{N}\left(\left\{B_{\left(G_{N}, \mathcal{O}_{N}\right)}(k) \simeq(g, o)\right\} \cap A\right) & \leq \mathbb{E}_{N, \gamma}^{b, p}\left(\mathbb{1}_{A} \cdot \frac{\#\left\{i: B_{\left(G_{N}, i\right)}(k) \simeq(g, o)\right\}}{N}\right) \\
& \leq \mathbb{P}_{N, \gamma}^{b, p}(A) \\
& <\frac{\delta}{3}
\end{aligned}
$$

and

$$
\begin{aligned}
\mathbb{P}_{N, \gamma}^{b, p} \otimes \mathcal{U}_{N}\left(\left\{B_{\left(G_{N}, \mathcal{O}_{N}\right)}(k) \simeq(g, o)\right\} \cap A^{c}\right) & =\mathbb{E}_{N, \gamma}^{b, p}\left(\mathbb{1}_{A^{c}} \cdot \frac{\#\left\{i: B_{\left(G_{N}, i\right)}(k) \simeq(g, o)\right\}}{N}\right) \\
& =\sum_{h \in A^{c}} \frac{\#\left\{i: B_{(h, i)}(k) \simeq(g, o)\right\}}{N} \cdot \mathbb{P}_{N, \gamma}^{b, p}(h) .
\end{aligned}
$$

Summing and subtracting $\mu_{\gamma}(k,(g, o)) \cdot \mathbb{P}_{N, \gamma}^{b, p}\left(A^{c}\right)$, and since every $h \in A^{c}$,

$$
\left|\frac{\#\left\{i: B_{(h, i)}(k) \simeq(g, o)\right\}}{N}-\mu_{\gamma}(k,(g, o))\right| \leq \frac{\delta}{3},
$$

we have

$$
\begin{aligned}
& \left|\sum_{h \in A^{c}} \frac{\#\left\{i: B_{(h, i)}(k) \simeq(g, o)\right\}}{N} \cdot \mathbb{P}_{N, \gamma}^{b, p}(h)-\mu_{\gamma}(k,(g, o))\right| \leq \\
& \leq\left|\sum_{h \in A^{c}} \frac{\#\left\{i: B_{(h, i)}(k) \simeq(g, o)\right\}}{N} \cdot \mathbb{P}_{N, \gamma}^{b, p}(h)-\mu_{\gamma}(k,(g, o)) \cdot \mathbb{P}_{N, \gamma}^{b, p}\left(A^{c}\right)\right|+\mu_{\gamma}(k,(g, o)) \cdot \mathbb{P}_{N, \gamma}^{b, p}(A) \\
& \leq \underbrace{\mu_{\gamma}(k,(g, o))}_{\leq 1} \cdot \underbrace{\mathbb{P}_{N, \gamma}^{b, p}(A)}_{<\delta / 3}+\frac{\delta}{3} \cdot \underbrace{\mathbb{P}_{N, \gamma}^{b, p}\left(A^{c}\right)}_{\leq 1} \\
& \leq \frac{2 \delta}{3} .
\end{aligned}
$$

Therefore, for $N$ sufficiently large,

$$
\left|\mathbb{P}_{N, \gamma}^{b, p} \otimes \mathcal{U}_{N}\left(\left\{B_{\left(G_{N}, \mathcal{O}_{N}\right)}(k) \simeq(g, o)\right\}\right)-\mu_{\gamma}(k,(g, o))\right| \leq \frac{2 \delta}{3}+\frac{\delta}{3}=\delta,
$$

as we desired.

\subsubsection{Estimates from [132]}

We now import some estimates that we will need from [132] (Lemmas 6 and 7 below) and state and prove a consequence of them (Corollary 4).

Lemma 6 ([132]). $\quad$ 1. Assume $\gamma \in(0,1)$. For each $\alpha \in(0,1)$ and $N$ large enough there exists a 
graph $\hat{g}_{\alpha, N}$ such that

$$
\begin{aligned}
& \mathcal{H}_{p}\left(\hat{g}_{\alpha, N}\right) \leq N^{\alpha} \text { for all } p \in[1, \infty] \text { and } \\
& \qquad \mathbb{P}_{N, \gamma}\left(\hat{g}_{\alpha, N} \text { is a subgraph of } G_{N}\right) \geq \exp \left\{-c N^{1-\alpha(1-\gamma)}\right\},
\end{aligned}
$$

where $c>0$ depends on $\gamma, \alpha$ but not on $N$.

2. Assume $\gamma=1$. For each $k \in \mathbb{N}$ and $N$ large enough there exists a graph $\hat{\hat{g}}_{k, N}$ such that

$$
\begin{aligned}
\mathcal{H}_{p}\left(\hat{\hat{g}}_{k, N}\right) \leq 3(k+1) N^{\frac{1}{k+1}} \text { for all } p \in[1, \infty] \text { and } \\
\mathbb{P}_{N, 1}\left(\hat{\hat{g}}_{k, N} \text { is a subgraph of } G_{N}\right) \geq \exp \{-k N\} .
\end{aligned}
$$

Lemma 7 ([132]). Assume $p \in[1, \infty], k \in \mathbb{N}$ and $\alpha<\frac{1}{k}$. There exists $\delta>0$ and a function $o_{1}(N)$ with $o_{1}(N) / N \stackrel{N \rightarrow \infty}{\longrightarrow} 0$ such that, for $N$ large enough,

$$
\text { if } g=([N], E) \in \mathcal{G} \text { with } \mathcal{H}_{p}(g) \leq N^{\alpha}, \text { then } \sum_{\substack{e \in E: \\|e| \geq N^{\delta}}}|e| \geq k N-o_{1}(N) \text {. }
$$

For the following corollary we will use Chernoff's inequality: Let $X$ be a random variable under a probability measure $\mathbb{P}$ and $t \in \mathbb{R}$. For every $\theta>0$,

$$
\mathbb{P}(X \geq t) \leq e^{-\theta t} \cdot \mathbb{E}\left(e^{\theta X}\right) .
$$

Corollary 4. 1. Assume $p \in[1, \infty]$ and either of the following conditions hold:

$$
[\gamma \in(0,1), b \in(-\infty, 1)] \text { or }[\gamma \geq 1, b<0] .
$$

If $E_{N}$ are events with $\mathbb{P}_{N, \gamma}\left(E_{N}\right)<\exp \{-\beta N\}$ for some $\beta>0$ and $N$ large, then

$$
\mathbb{P}_{N, \gamma}^{b, p}\left(E_{N}\right) \stackrel{N \rightarrow \infty}{\longrightarrow} 0 \text {. }
$$

2. Assume $\gamma=1, p \in[1, \infty]$ and $b \in[0,1) \backslash \mathcal{E}_{p}$. Then,

2a. there exists $C>0$ such that, if $E_{N}$ are events with $\mathbb{P}_{N, \gamma}\left(E_{N}\right)<\exp \{-C N\}$ for all $N$, then

$$
\mathbb{P}_{N, 1}^{b, p}\left(E_{N}\right) \stackrel{N \rightarrow \infty}{\longrightarrow} 0 ;
$$

2b. if $E_{N}$ are events such that $\mathbb{P}_{N, \gamma}\left(E_{N}\right)<\exp \{-c N\}$ for some $c>0$, and each $E_{N}$ only depends on $\{e:|e| \leq L\}$ for a fixed $L$, then

$$
\mathbb{P}_{N, 1}^{b, p}\left(E_{N}\right) \stackrel{N \rightarrow \infty}{\longrightarrow} 0 .
$$

Proof. We start assuming that

$$
p \in[1, \infty], \gamma>0, b<0 .
$$

In this case, using that $\mathcal{H}_{p}(g) \leq N$ for every $g \in \mathcal{G}$,

$$
Z_{N, \gamma}^{b, p}=\sum_{\substack{g \in \mathcal{G} \\ V(g)=\{1, \ldots, N\}}} \exp \left\{-N^{b} \cdot \mathcal{H}_{p}(g)\right\} \cdot \mathbb{P}_{N, \gamma}(g) \geq \exp \left\{-N^{b+1}\right\} .
$$

Thus, by the fact that $b<0$,

$$
\mathbb{P}_{N, \gamma}^{b, p}\left(E_{N}\right) \leq\left(Z_{N, \gamma}^{b, p}\right)^{-1} \cdot \mathbb{P}_{N, \gamma}\left(E_{N}\right) \leq \exp \left\{-\beta N+N^{b+1}\right\} \stackrel{N \rightarrow \infty}{\longrightarrow} 0 .
$$


This proves part of statement 1 of the corollary; to complete the proof of statement 1 , we now assume

$$
p \in[1, \infty], \gamma \in(0,1), b \in(-1+\gamma, 1) .
$$

Applying Lemma 6 , for any $\alpha \in(0,1)$ we obtain the following lower bound for the partition function, for any $\alpha \in(0,1)$ :

$$
\begin{aligned}
& Z_{N, \gamma}^{b, p} \geq \sum_{\substack{g \in \mathcal{G} \\
V(g)=\{1, \ldots, N\} \\
\hat{g}_{\alpha, N} \text { is a subgraph of } g}} \exp \left\{-N^{b} \cdot \mathcal{H}_{p}(g)\right\} \cdot \mathbb{P}_{N, \gamma}(g) \\
& \mathcal{H}_{p}\left(\hat{g}_{\alpha, N}\right) \leq \mathcal{H}_{p}(g) \rightarrow \geq \exp \left\{-N^{b} \cdot \mathcal{H}_{p}\left(\hat{g}_{\alpha, N}\right)\right\} \cdot \mathbb{P}_{N, \gamma}\left(\hat{g}_{\alpha, N} \text { is a subgraph of } G_{N}\right) \\
& \text { Lemma } 6 \rightarrow \geq \exp \left\{-N^{b+\alpha}-c N^{1-\alpha(1-\gamma)}\right\} .
\end{aligned}
$$

Thus,

$$
\mathbb{P}_{N, \gamma}^{b, p}\left(E_{N}\right) \leq\left(Z_{N, \gamma}^{b, p}\right)^{-1} \cdot \mathbb{P}_{N, \gamma}\left(E_{N}\right) \leq \exp \left\{-\beta N+N^{b+\alpha}+c N^{1-\alpha(1-\gamma)}\right\}
$$

Since $b<1$, setting $\alpha=\frac{1-b}{2-\gamma}$ gives

$$
b+\alpha=b+\frac{1-b}{2-\gamma}=\frac{b(1-\gamma)+1}{2-\gamma}<1
$$

and $1-\alpha(1-\gamma)<1$, proving (6.20).

Now suppose $\gamma=1, p \in[1, \infty]$ and $b \in[0,1) \backslash \mathcal{E}_{p}$. Let $k \in \mathbb{N}$ be the unique integer such that

$$
\frac{k-1}{k}<b<\frac{k}{k+1} \text {. }
$$

From Lemma 6, we have

$$
\begin{aligned}
& Z_{N, 1}^{b, p} \geq \sum_{\substack{g \in \mathcal{G} \\
V(g)=\{1, \ldots, N\}}} \exp \left\{-N^{b} \cdot \mathcal{H}_{p}(g)\right\} \cdot \mathbb{P}_{N, \gamma}(g) \\
& \hat{\hat{g}}_{k, N} \text { is a subgraph of } g \\
& \mathcal{H}_{p}\left(\hat{\hat{g}}_{k, N}\right) \leq \mathcal{H}_{p}(g) \rightarrow \geq \exp \left\{-N^{b} \cdot \mathcal{H}_{p}\left(\hat{\hat{g}}_{k, N}\right)\right\} \cdot \mathbb{P}_{N, \gamma}\left(\hat{\hat{g}}_{k, N} \text { is a subgraph of } G_{N}\right) \\
& \text { Lemma } 6 \rightarrow \geq \exp \left\{-3(k+1) N^{b+\frac{1}{k+1}}-k N\right\} .
\end{aligned}
$$

Then

$$
Z_{N, 1}^{b, p} \geq \exp \{-k N+o(N)\},
$$

where $o(N) / N \rightarrow 0$. The last equality holds by (6.24). Then, for $C=C(b)>k$, we have

$$
\mathbb{P}_{N, \gamma}^{b, p}\left(E_{N}\right) \leq\left(Z_{N, \gamma}^{b, p}\right)^{-1} \cdot \mathbb{P}_{N, \gamma}\left(E_{N}\right) \leq \exp \{-C N+k N-o(N)\} \stackrel{N \rightarrow \infty}{\longrightarrow} 0,
$$

proving (6.21). To prove (6.22), fix an arbitrary $\frac{1}{k+1}<\alpha<\frac{1}{k}$. Take $\delta>0$ and $o_{1}(N)$ as in Lemma 7 .

Define

$$
\begin{aligned}
& B=\left\{\mathcal{H}_{p}\left(G_{N}\right) \leq N^{\alpha}\right\} \text { and } \\
& C=\left\{G_{N}=([N], E): \sum_{e \in E:|e| \geq N^{\delta}}|e| \geq k N-o_{1}(N)\right\} .
\end{aligned}
$$

Then,

$$
\mathbb{P}_{N, 1}^{b, p}\left(E_{N}\right)=\mathbb{P}_{N, 1}^{b, p}\left(E_{N} \cap B\right)+\mathbb{P}_{N, 1}^{b, p}\left(E_{N} \cap B^{c}\right)
$$


Choose $\varepsilon>0$ such that

$$
\frac{1}{k+1}+\varepsilon<\alpha
$$

From Theorem 41, since $b$ satisfies (6.24),

$$
\mathbb{P}_{N, 1}^{b, p}\left(N^{\frac{1}{k+1}-\varepsilon}<\mathcal{H}_{p}\left(G_{N}\right)<N^{\frac{1}{k+1}+\varepsilon}\right) \stackrel{N \rightarrow \infty}{\longrightarrow} 1 .
$$

Thus,

$$
\mathbb{P}_{N, 1}^{b, p}\left(E_{N} \cap B^{c}\right) \leq \mathbb{P}_{N, 1}^{b, p}\left(\mathcal{H}_{p}\left(G_{N}\right)>N^{\alpha}\right) \stackrel{N \rightarrow \infty}{\longrightarrow} 0 .
$$

Lemma 7 claims that $B \subseteq C$. Moreover, since the event $E_{N}$ depends only on edges with length at most $L$, we can take $N$ large enough so that the events $E_{N}$ and $C$ are independent under the reference measure $\mathbb{P}_{N, 1}$. Thus,

$$
\begin{aligned}
\mathbb{P}_{N, 1}^{b, p}\left(E_{N} \cap B\right) & \leq \mathbb{P}_{N, 1}^{b, p}\left(E_{N} \cap C\right) \\
& \leq\left(Z_{N, 1}^{b, p}\right)^{-1} \cdot \mathbb{P}_{N, 1}\left(E_{N}\right) \cdot \mathbb{P}_{N, 1}(C)
\end{aligned}
$$

By $(6.25) \rightarrow \leq \exp \{k N-o(N)\} \cdot \exp \{-c N\} \cdot \mathbb{P}_{N, 1}(C)$.

Now, by Chernoff's inequality,

$$
\begin{aligned}
& \mathbb{P}_{N, 1}(C)=\mathbb{P}_{N, 1}\left(\sum_{e \in E\left(G_{N}\right):|e| \geq N^{\delta}}|e| \geq k N-o_{1}(N)\right) \\
& \text { Chernoff's inequality } \rightarrow \leq \exp \left\{-\theta\left(k N-o_{1}(N)\right)\right\} \cdot \mathbb{E}_{N, 1}\left(\exp \left\{\theta \sum_{e \in E\left(G_{N}\right):|e| \geq N^{\delta}}|e|\right\}\right) \\
& \text { Independence } \rightarrow=\exp \left\{-\theta\left(k N-o_{1}(N)\right)\right\} \cdot \prod_{\substack{e \in \mathcal{E}_{N}: \\
|e| \geq N^{\delta}}} \mathbb{E}_{N, 1}\left(\exp \left\{\theta \cdot|e| \cdot \mathbb{1}_{\left\{e \in E\left(G_{N}\right)\right\}}\right\}\right) \\
& \leq \exp \left\{-\theta\left(k N-o_{1}(N)\right)\right\} \cdot \prod_{\substack{e \in \mathcal{E}_{N}: \\
|e| \geq N^{\delta}}}(1+\exp \{(\theta-1)|e|\}) \\
& \text { Using } x+1 \leq e^{x} \rightarrow \leq \exp \left\{-\theta\left(k N-o_{1}(N)\right)\right\} \cdot \prod_{\substack{e \in \mathcal{E}_{N}: \\
|e| \geq N^{\delta}}} \exp \{\exp \{(\theta-1)|e|\}\} \\
& \leq \exp \left\{-\theta\left(k N-o_{1}(N)\right)\right\} \cdot \prod_{i=\left\lfloor N^{\delta}\right\rfloor}^{N} \prod_{\substack{e \in \mathcal{E}_{N}: \\
|e|=i}} \exp \{\exp \{(\theta-1) i\}\} \\
& \leq \exp \left\{-\theta\left(k N-o_{1}(N)\right)+\sum_{i=\left\lfloor N^{\delta}\right\rfloor}^{N} \sum_{\substack{e \in \mathcal{E}_{N}: \\
|e|=i}} \exp \{(\theta-1) i\}\right\} \\
& \#\left\{e \in \mathcal{E}_{N}:|e|=i\right\} \leq N \rightarrow \leq \exp \left\{-\theta\left(k N-o_{1}(N)\right)+N \sum_{i=\left\lfloor N^{\delta}\right\rfloor}^{\infty} \exp \{(\theta-1) i\}\right\} \text {. }
\end{aligned}
$$

Taking any $\delta^{\prime}<\delta$, and $\theta=1-N^{-\delta^{\prime}}$, for $N$ large enough,

$$
N \sum_{i=\left\lfloor N^{\delta}\right\rfloor}^{\infty} \exp \{(\theta-1) i\}=N \sum_{i=\left\lfloor N^{\delta}\right\rfloor}^{\infty} \exp \left\{-N^{-\delta^{\prime}} i\right\} \leq 2 .
$$


Thus,

$$
\mathbb{P}_{N, 1}(C) \leq \exp \left\{-\left(1-N^{-\delta^{\prime}}\right)\left(k N-o_{1}(N)\right)+2\right\}=\exp \{-k N+o(N)\} .
$$

This completes the proof of $(6.22)$.

\subsubsection{Estimates for the reference measure and proof of Proposition 19}

We will use the following concentration result [103] for sums of bounded random variables with finite-range dependence. Let $(\Omega, \mathcal{F}, \mathbb{P})$ be a probability space, $\mathcal{A}$ be some index set and $\left\{Y_{\alpha}\right\}_{\alpha \in \mathcal{A}}$ be a family of random variables.

- A subset $\mathcal{A}^{\prime}$ of $\mathcal{A}$ is independent if the corresponding random variables $\left\{Y_{\alpha}\right\}_{\alpha \in \mathcal{A}^{\prime}}$, are independent.

- A family $\left\{\mathcal{A}_{j}\right\}_{j}$ of subsets of $\mathcal{A}$ is a cover of $\mathcal{A}$ if $\bigcup_{j} \mathcal{A}_{j}=\mathcal{A}$.

- A family $\left\{\left(\mathcal{A}_{j}, w_{j}\right)\right\}_{j}$ of pairs $\left(\mathcal{A}_{j}, w_{j}\right)$, where $\mathcal{A}_{j}$ is a subset of $\mathcal{A}$ and $w_{j} \in[0,1]$ is a fractional cover of $\mathcal{A}$ if $\sum_{j: \alpha \in \mathcal{A}_{j}} w_{j} \geq 1$ for each $\alpha \in \mathcal{A}$.

- A (fractional) cover is proper if each set $\mathcal{A}_{j}$ is independent.

- $\chi(\mathcal{A})$ is the size of the smallest proper cover of $\mathcal{A}$, i.e. the smallest $m$ such that $\mathcal{A}$ is the union of $m$ independent subsets (in combinatory, this number is the chromatic number of the set $\mathcal{A}$ ).

- $\chi^{*}(\mathcal{A})$ is the minimum of $\sum_{j} w_{j}$ over all proper fractional covers $\left\{\left(\mathcal{A}_{j}, w_{j}\right)\right\}_{j}$.

Note that, taking $w_{j}=1$ for every $j$, we have $\chi^{*}(\mathcal{A}) \leq \chi(\mathcal{A})$. The following theorem is the main result in [103].

Theorem 43. Let $(\Omega, \mathcal{F}, \mathbb{P})$ be a probability space, and $X$ be the random variable $X=\sum_{\alpha \in \mathcal{A}} Y_{\alpha}$ of a family of random variables $Y_{\alpha}$, with $\alpha$ ranging over some index set $\mathcal{A}$, and with $a_{\alpha} \leq Y_{\alpha} \leq b_{\alpha}$ for every $\alpha \in \mathcal{A}$ and some real numbers $a_{\alpha}$ and $b_{\alpha}$. Then, for $t>0$,

$$
\mathbb{P}(X \geq \mathbb{E}(X)+t) \leq \exp \left(-2 \frac{t^{2}}{\chi^{*}(\mathcal{A}) \sum_{\alpha \in \mathcal{A}}\left(b_{\alpha}-a_{\alpha}\right)^{2}}\right) .
$$

As said in [103], Theorem 43 extends Theorem 2 of [94], which is the case of independent variables (and so $\chi^{*}(\mathcal{A})=1$ ). We will use a particular case of Theorem 43 .

Lemma 8. Let $Y_{1}, \ldots, Y_{n}$ be random variables such that, for some $m, L>0$ and for each $i$, $0 \leq Y_{i} \leq m$ and $Y_{i}$ is independent of $\left\{Y_{j}:|j-i|>L\right\}$. Then, letting $X=\sum_{i=1}^{n} Y_{i}$, we have

$$
\mathbb{P}(|X-\mathbb{E}(X)|>t) \leq 2 \exp \left\{-\frac{2 t^{2}}{(2 L+1) n m^{2}}\right\} .
$$

We now state and prove two lemmas which give upper bounds to the probabilities of the same events that appear in the two parts of Proposition 19; however, in these lemmas, the probability measure under consideration is the reference measure $\mathbb{P}_{N, \gamma}$ rather than the weighted measure $\mathbb{P}_{N, \gamma}^{b, p}$.

Lemma 9. Let $\gamma>0, k, L>0,(g, o) \in \mathcal{G} \bullet$ and $\varepsilon>0$. For $N$ large enough we have

$$
\mathbb{P}_{N, \gamma}\left(\left|\frac{\#\left\{i \in[N]: B_{\left(G_{N}, i\right)}^{L}(k) \simeq(g, o)\right\}}{N}-\mu_{\gamma}^{L}(k,(g, o))\right|>\varepsilon\right)<2 \exp \left\{-\frac{\varepsilon^{2} N}{8 k L+2}\right\}
$$

where $\mu_{\gamma}^{L}(k,(g, o))$ is as in $(6.12)$. 
Proof. Fix $\gamma, k, L,(g, o)$ and $\varepsilon$. Also let $N>L$. Define

$$
\begin{aligned}
& Y_{N, i}=N^{-1} \cdot \mathbb{1}\left\{B_{(G, i)}^{L}(k) \simeq(g, o)\right\}, i \in[N], \\
& X_{N}=\sum_{i=1}^{N} Y_{N, i}, \quad \nu_{N, \gamma}^{L}(k,(g, o))=\mathbb{E}_{N, \gamma}\left(X_{N}\right) .
\end{aligned}
$$

If $1+k L<i<N-k L$, then

$$
\mathbb{P}_{N, \gamma}\left(B_{\left(G_{N}, i\right)}^{L}(k) \simeq(g, o)\right)=\mu_{\gamma}^{L}(k,(g, o)),
$$

so, we have

$$
\begin{aligned}
\left|\nu_{N, \gamma}^{L}(k,(g, o))-\mu_{\gamma}^{L}(k,(g, o))\right| & =\left|\sum_{i=1}^{N} N^{-1} \mathbb{P}_{N, \gamma}\left(B_{\left(G_{N}, i\right)}^{L}(k) \simeq(g, o)\right)-\mu_{\gamma}^{L}(k,(g, o))\right| \\
& \leq\left|\sum_{i=1+k L}^{N-k L} N^{-1} \mu_{\gamma}^{L}(k,(g, o))-\mu_{\gamma}^{L}(k,(g, o))\right|+\frac{2 k L}{N} \\
& =\frac{2 k L}{N} \cdot \mu_{\gamma}^{L}(k,(g, o))+\frac{2 k L}{N},
\end{aligned}
$$

and then, for $N$ large enough,

$$
\left|\nu_{N, \gamma}^{L}(k,(g, o))-\mu_{\gamma}^{L}(k,(g, o))\right|<\frac{\varepsilon}{2} .
$$

Moreover, for each $i \in[N]$ we have $0 \leq Y_{N, i} \leq N^{-1}$ and $Y_{N, i}$ is independent of $\left\{Y_{N, j}:|j-i|>2 k L\right\}$ under $\mathbb{P}_{N, \gamma}$, so Lemma 8 yields

$$
\begin{aligned}
\mathbb{P}_{N, \gamma}\left(\left|X_{N}-\nu_{N, \gamma}^{L}(k,(g, o))\right|>\frac{\varepsilon}{2}\right) & \leq 2 \exp \left\{-\frac{2 \varepsilon^{2} N^{2}}{4(4 k L+1) N}\right\} \\
& =2 \exp \left\{-\frac{\varepsilon^{2} N}{8 k L+2}\right\} .
\end{aligned}
$$

The result now follows from (6.29) and (6.30) and by the triangle inequality, since for $N$ sufficiently large,

$$
\begin{aligned}
\left|X_{N}-\mu_{\gamma}^{L}(k,(g, o))\right| & \leq\left|X_{N}-\nu_{N, \gamma}^{L}(k,(g, o))\right|+\left|\nu_{N, \gamma}^{L}(k,(g, o))-\mu_{\gamma}^{L}(k,(g, o))\right| \\
& \leq\left|X_{N}-\nu_{N, \gamma}^{L}(k,(g, o))\right|+\frac{\varepsilon}{2},
\end{aligned}
$$

and thus,

$$
\begin{aligned}
\mathbb{P}_{N, \gamma}\left(\left|X_{N}-\mu_{\gamma}^{L}(k,(g, o))\right|>\varepsilon\right) & \leq \mathbb{P}_{N, \gamma}\left(\left|X_{N}-\nu_{N, \gamma}^{L}(k,(g, o))\right|>\frac{\varepsilon}{2}\right) \\
& \leq 2 \exp \left\{-\frac{\varepsilon^{2} N}{8 k L+2}\right\}
\end{aligned}
$$

as we desired.

Lemma 10. For every $\gamma>0, N>1$ and $\varepsilon>0$ we have, for $L$ large enough,

$$
\mathbb{P}_{N, \gamma}\left(G_{N} \text { has more than } \varepsilon N \text { edges with length larger than } L\right) \leq \exp \left\{-L^{\gamma / 8} N\right\} \text {. }
$$

Proof. Fix $L \geq 1$ and $g \in \mathcal{G}$ with $V(g)=\{1, \ldots, N\}$, and define

$$
\begin{aligned}
\Lambda_{N, L} & =\{\{i, j\}: 1 \leq i<i+L<j \leq N\}, \\
\Lambda_{N, L, g} & =\Lambda_{N, L} \cap E(g) .
\end{aligned}
$$


By Chernoff's inequality, for $\theta>0$,

$\mathbb{P}_{N, \gamma}\left(G_{N}\right.$ has more than $\varepsilon N$ edges with length larger than $\left.L\right) \leq$ Chernoff's inequality $\rightarrow \leq \exp \{-\theta \varepsilon N\} \cdot \mathbb{E}_{N, \gamma}\left(\exp \left\{\theta \cdot \#\left\{\{i, j\} \in \Lambda_{N, L, G_{N}}\right\}\right\}\right)$

$$
=\exp \{-\theta \varepsilon N\} \cdot \mathbb{E}_{N, \gamma}\left(\prod_{\{i, j\} \in \Lambda_{N, L}} \exp \left\{\theta \cdot \mathbb{1}_{\left\{\{i, j\} \in E\left(G_{N}\right)\right\}}\right\}\right)
$$

$$
\text { Independence } \rightarrow \leq \exp \{-\theta \varepsilon N\} \prod_{\{i, j\} \in \Lambda_{N, L}}\left(p_{\{i, j\}} \exp \{\theta\}+1-p_{\{i, j\}}\right)
$$

$$
\text { Using } x+1 \leq e^{x} \rightarrow \leq \exp \{-\theta \varepsilon N\} \prod_{\{i, j\} \in \Lambda_{N, L}} \exp \left\{p_{\{i, j\}}(\exp \{\theta\}-1)\right\} \text {; }
$$

we remind the reader that $p_{\{i, j\}}=\exp \left\{-|j-i|^{\gamma}\right\}$. The right-hand side is less than

$$
\begin{aligned}
& \exp \{-\theta \varepsilon N\} \prod_{\{i, j\} \in \Lambda_{N, L}} \exp \left\{p_{\{i, j\}}(\exp \{\theta\}-1)\right\} \\
& \leq \exp \left\{-\theta \varepsilon N+(\exp \{\theta\}-1) \sum_{\{i, j\} \in \Lambda_{N, L}} p_{\{i, j\}}\right\} .
\end{aligned}
$$

We then bound

$$
\sum_{\{i, j\} \in \Lambda_{N, L}} p_{\{i, j\}}=\sum_{k=L+1}^{N}(N-k+1) \exp \left\{-k^{\gamma}\right\} \leq N \sum_{k=L+1}^{\infty} \exp \left\{-k^{\gamma}\right\} .
$$

Then,

$$
\begin{aligned}
\sum_{k=L+1}^{\infty} \exp \left\{-k^{\gamma}\right\} & \leq \exp \left\{-L^{\gamma}\right\} \sum_{k=L+1}^{\infty} \exp \left\{-k^{\gamma}+L^{\gamma}\right\} \\
& \leq \exp \left\{-L^{\gamma}\right\} \sum_{j=1}^{\infty} \sum_{k=\lfloor j L\rfloor}^{\lceil(j+1) L\rceil} \exp \left\{-k^{\gamma}+L^{\gamma}\right\} \\
& \leq \exp \left\{-L^{\gamma}\right\} \sum_{j=1}^{\infty} 2 L \exp \left\{-(j L)^{\gamma}+L^{\gamma}\right\} \\
& \leq 2 L \exp \left\{-L^{\gamma}\right\} \sum_{j=1}^{\infty} \exp \left\{-L^{\gamma}\left(j^{\gamma}-1\right)\right\} \\
& \leq 2 \cdot L \cdot \exp \left\{-L^{\gamma}\right\} \cdot C_{\gamma}
\end{aligned}
$$

where $C_{\gamma}=\sum_{j=1}^{\infty} \exp \left\{-\left(j^{\gamma}-1\right)\right\}$. Thus, for $L$ large enough,

$$
\sum_{\{i, j\} \in \Lambda_{N, L}} p_{\{i, j\}}<N \exp \left\{-L^{\gamma / 2}\right\} .
$$

Choosing $\theta=L^{\frac{\gamma}{4}}$, the expression in (6.31) is at most

$$
\exp \left\{-L^{\frac{\gamma}{4}} \varepsilon N+\exp \left\{L^{\frac{\gamma}{4}}\right\} \cdot \exp \left\{-L^{\frac{\gamma}{2}}\right\} \cdot N\right\}<\exp \left\{-L^{\frac{\gamma}{8}} N\right\}
$$

if $L$ is large enough.

Proof of Proposition 19. For $0<\gamma<1, b \in(-\infty, 1)$ or $\gamma \geq 1, b<0$, by Lemma 9 and 10, and by 
Corollary 4, item 1, we conclude (6.11) and (6.13). For $\gamma=1, b \in(0,1) \backslash \mathcal{E}_{p}$, since the event

$$
\left\{\left|\frac{\#\left\{i \in[N]: B_{\left(G_{N}, i\right)}^{L}(k) \simeq(g, o)\right\}}{N}-\mu_{\gamma}^{L}(k,(g, o))\right|>\varepsilon\right\}
$$

depends only of edges with length at most $L$, we can apply Corollary 4 , item $2 \mathrm{~b}$, to conclude (6.11). Finally, choosing $L \geq 1$ such that $L^{\gamma / 8}>C$, where $C>0$ is the constant of Corollary 4, item 2a, by Lemma 10 we conclude (6.13).

\subsection{No local convergence for $\gamma>1, p<\infty$ and $b>p+1$}

Proof of Proposition 18. Fix $\gamma>1, p \in[1, \infty), b>p+1$ and $L>1$. Also fix $N \in \mathbb{N}$. For $g=([N], E) \in \mathcal{L}_{L}$ and $k \in \mathbb{N}$, let $M_{L, k}(g)$ be the set of graphs obtained by removing $k$ edges with length at most $L$ from $g$. For $g^{\prime} \in M_{L, k}(g)$, consider $\tilde{E}_{k}=\left\{\left\{x_{1}, y_{1}\right\}, \ldots,\left\{x_{k}, y_{k}\right\}\right\}$ be the removed edges from $g$. Since $\mathrm{d}_{g^{\prime}}(x, y) \geq \mathrm{d}_{g}(x, y)$ for every $x, y \in V$, and $\mathrm{d}_{g^{\prime}}\left(x_{l}, y_{l}\right) \geq \mathrm{d}_{g}\left(x_{l}, y_{l}\right)+1$ for every $l=1 \ldots, k$, we have

$$
\sum_{l=1}^{k}\left(\mathrm{~d}_{g^{\prime}}\left(x_{l}, y_{l}\right)\right)^{p} \geq \sum_{l=1}^{k} \sum_{t=0}^{p}\left(\begin{array}{l}
p \\
t
\end{array}\right)\left(\mathrm{d}_{g}\left(x_{l}, y_{l}\right)\right)^{t} .
$$

Then,

$$
\begin{aligned}
\left(\mathcal{H}_{p}\left(g^{\prime}\right)\right)^{p} & =\frac{1}{\left(\begin{array}{c}
N \\
2
\end{array}\right)} \sum_{\substack{x, y \in V \\
x<y \\
\{x, y\} \notin \tilde{E}_{k}}}\left(\mathrm{~d}_{g^{\prime}}(x, y)\right)^{p}+\frac{1}{\left(\begin{array}{c}
N \\
2
\end{array}\right)} \sum_{l=1}^{k}\left(\mathrm{~d}_{g^{\prime}}\left(x_{l}, y_{l}\right)\right)^{p} \\
& \geq \frac{1}{\left(\begin{array}{c}
N \\
2
\end{array}\right)} \sum_{\substack{x, y \in V \\
x<y}}\left(\mathrm{~d}_{g^{\prime}}(x, y)\right)^{p}+\frac{1}{\left(\begin{array}{c}
N \\
2
\end{array}\right)} \sum_{l=1}^{k} \sum_{t=0}^{p-1}\left(\begin{array}{c}
p \\
t
\end{array}\right)\left(\mathrm{d}_{g}\left(x_{l}, y_{l}\right)\right)^{t} \\
\mathrm{~d}_{g}\left(x_{l}, y_{l}\right) \geq 1 \rightarrow & =\frac{1}{\left(\begin{array}{c}
N \\
2
\end{array}\right)} \sum_{\substack{x, y \in V \\
x<y}}\left(\mathrm{~d}_{g}(x, y)\right)^{p}+\frac{k}{\left(\begin{array}{c}
N \\
2
\end{array}\right)} \cdot\left(2^{p}-1\right) .
\end{aligned}
$$

Thus,

$$
\left(\mathcal{H}_{p}\left(g^{\prime}\right)\right)^{p} \geq\left(\mathcal{H}_{p}(g)\right)^{p}+\frac{k}{\left(\begin{array}{c}
N \\
2
\end{array}\right)} \cdot\left(2^{p}-1\right) .
$$

Since $\mathcal{H}_{p}(g) \in[1, N]$, the mean value theorem gives

$$
\mathcal{H}_{p}\left(g^{\prime}\right)-\mathcal{H}_{p}(g) \geq \frac{\left(\mathcal{H}_{p}\left(g^{\prime}\right)\right)^{p}-\left(\mathcal{H}_{p}(g)\right)^{p}}{p \cdot N^{p-1}} \geq \frac{2^{p}-1}{p} \cdot \frac{k}{N^{p+1}} .
$$

Thus, for $g^{\prime} \in M_{L, k}(g)$,

$$
\frac{\mathbb{P}_{N, \gamma}\left(g^{\prime}\right)}{\mathbb{P}_{N, \gamma}(g)}=\prod_{l=1}^{k} \exp \left\{\left|x_{l}-y_{l}\right|^{\gamma}\right\} \leq \prod_{l=1}^{k} \exp \left\{L^{\gamma}\right\}=\exp \left\{L^{\gamma} k\right\},
$$

and then,

$$
\begin{aligned}
\frac{\mathbb{P}_{N, \gamma}^{b, p}\left(G_{N}=g^{\prime}\right)}{\mathbb{P}_{N, \gamma}^{b, p}\left(G_{N}=g\right)} & =\exp \left\{-N^{b}\left(\mathcal{H}_{p}\left(g^{\prime}\right)-\mathcal{H}_{p}(g)\right)\right\} \frac{\mathbb{P}_{N, \gamma}\left(g^{\prime}\right)}{\mathbb{P}_{N, \gamma}\left(g^{\prime}\right)} \\
& \leq \exp \left\{-k N^{b-p-1} \cdot \frac{\left(2^{p}-1\right)}{p}\right\}\left(\exp \left\{L^{\gamma}\right\}\right)^{k} .
\end{aligned}
$$


Noting that the number of edges with length at most $L$ in $\{1, \ldots, N\}$ is at most $N L$, and

$$
\# M_{L, k}(g) \leq\left(\begin{array}{c}
N L \\
k
\end{array}\right)
$$

we bound

$$
\begin{gathered}
\sum_{k=1}^{N L} \sum_{g^{\prime} \in M_{L, k}(g)} \frac{\mathbb{P}_{N, \gamma}^{b, p}\left(G_{N}=g^{\prime}\right)}{\mathbb{P}_{N, \gamma}^{b, p}\left(G_{N}=g\right)} \leq \sum_{k=1}^{N L}\left(\begin{array}{c}
N L \\
k
\end{array}\right) \exp \left\{-k N^{b-p-1} \cdot \frac{\left(2^{p}-1\right)}{p}\right\}\left(\exp \left\{L^{\gamma}\right\}\right)^{k} \\
\leq \sum_{k=1}^{\infty}\left(\exp \left\{L^{\gamma}\right\} \cdot N \cdot L \cdot \exp \left\{-N^{b-p-1} \cdot \frac{\left(2^{p}-1\right)}{p}\right\}\right)^{k} \stackrel{N \rightarrow \infty}{\longrightarrow} 0
\end{gathered}
$$

since $b>p+1$. Moreover, note that the family $\left\{M_{L, k}(g)\right\}_{k \geq 1, g \in \mathcal{L}_{L}}$ is pairwise disjoint, and

$$
\bigcup_{g \in \mathcal{L}_{L}} \bigcup_{k \geq 1} M_{L, k}(g)=\mathcal{L}_{L}^{c}
$$

Thus,

$$
\mathbb{P}_{N, \gamma}^{b, p}\left(G_{N} \notin \mathcal{L}_{L}\right) \leq \sum_{g \in \mathcal{L}_{L}} \mathbb{P}_{N, \gamma}^{b, p}\left(G_{N}=g\right) \sum_{k=1}^{N L} \sum_{g^{\prime} \in M_{L, k}(g)} \frac{\mathbb{P}_{N, \gamma}^{b, p}\left(G_{N}=g^{\prime}\right)}{\mathbb{P}_{N, \gamma}^{b, p}\left(G_{N}=g\right)} \stackrel{N \rightarrow \infty}{\longrightarrow} 0,
$$

as desired.

For the case $p=\infty$, it is possible to prove a weak statement that evidences the possibility of the sequence $\left(G_{N}, \mathcal{O}_{N}\right)$ sampled from $\mathbb{P}_{N, \gamma}^{b, \infty} \otimes \mathcal{U}_{N}$ does not converge in distribution.

Given a graph $g \in \mathcal{G}$ and $L>1$, we denote by $\hat{g}_{L} \in \mathcal{L}_{L}$ to be the graph obtained by modifying $g$ so that all edges $e$ with length $|e| \leq L$ are present. Define $\mathcal{H}_{L}^{*}(g):=\mathcal{H}_{\infty}\left(\hat{g}_{L}\right)$, and note that, for every $g$, we have $\mathcal{H}_{\infty}(g) \geq \mathcal{H}_{L}^{*}(g)$. We say that $g$ is $L$-optimal if

$$
\mathcal{H}_{\infty}(g)=\mathcal{H}_{L}^{*}(g)
$$

Theorem 44. Let $\gamma>1$ and $b>1$. For every $L>1$, we have

$$
\mathbb{P}_{N, \gamma}^{b, \infty}\left(G_{N} \text { is L-optimal }\right) \stackrel{N \rightarrow \infty}{\longrightarrow} 1 .
$$

Proof. Fix $N \geq 1, \gamma>1, b>1$ and $L>1$. Note that, if $g$ is not $L$-optimal, then

$$
\mathcal{H}_{\infty}(g) \geq \mathcal{H}_{L}^{*}(g)+1 .
$$

Let $\mathcal{P}_{L}$ be the set of all $L$-optimal graphs, and consider $\mathcal{G}^{L+}$ (resp. $\mathcal{G}^{L-}$ ) be the set of graphs $g=([N], E)$ containing only edges $e$ with length $|e|>L$ (resp. $|e| \leq L)$ and all edges $\{x, y\}$ with $|x-y|=1$. Note that the sets

$$
\mathcal{G}_{N}=\{g \in \mathcal{G}: g=([N], E), E \supset\{\{x, y\}: x, y \in[N],|x-y|=1\}\} \quad \text { and } \quad \mathcal{G}^{L+} \times \mathcal{G}^{L-}
$$

are isomorphic. In fact, the function $\psi: \mathcal{G}_{N} \rightarrow \mathcal{G}^{L+} \times \mathcal{G}^{L-}$ defined by $\psi(g)=\left(g^{+}, g^{-}\right)$, where $g^{+}$is the graph obtained by $g$ removing all edges with length $1<|e| \leq L$, and $g^{-}$is the graph obtained by $g$ removing all edges with length $|e|>L$, is a bijection. Define $H_{\infty}(\psi(g)):=H_{\infty}(g)$ and $\mathbb{P}_{N, \gamma}(\psi(g)):=\mathbb{P}_{N, \gamma}(g)$. Consider $k_{L} \in \mathcal{L}_{L}$ be the graph on $[N]$ such that all edges with length at most $L$ are present, and all edges with length larger than $L$ are absent. Thus,

$$
\mathbb{P}_{N, \gamma}^{b, \infty}\left(\mathcal{P}_{L}^{c}\right)=\frac{1}{Z_{N, \gamma}^{b, \infty}} \sum_{g \in \mathcal{P}_{L}^{c}} \exp \left\{-N^{b} \cdot \mathcal{H}_{\infty}(g)\right\} \mathbb{P}_{N, \gamma}(g)
$$




$$
\begin{aligned}
& =\frac{1}{Z_{N, \gamma}^{b, \infty}} \sum_{\left(g^{+}, g^{-}\right) \in \psi\left(\mathcal{P}_{L}^{c}\right)} \exp \left\{-N^{b} \cdot\left(\mathcal{H}_{L}^{*}\left(g^{+}, g^{-}\right)+1\right)\right\} \mathbb{P}_{N, \gamma}\left(g^{+}, g^{-}\right) \\
& \leq \frac{1}{Z_{N, \gamma}^{b, \infty}} \sum_{g^{+} \in \mathcal{G}^{L+}} \exp \left\{-N^{b} \cdot\left(H_{L}^{*}\left(g^{+}, k_{L}\right)+1\right)\right\} \sum_{\substack{g^{-} \in \mathcal{G}^{L-} \\
\left(g^{+}, g^{-}\right) \in \psi\left(\mathcal{P}_{L}^{c}\right)}} \mathbb{P}_{N, \gamma}\left(g^{+}, g^{-}\right) .
\end{aligned}
$$

Since $\# \mathcal{G}^{L-} \leq 2^{N L}$, we have

$$
\begin{aligned}
\sum_{\substack{g^{-} \in \mathcal{G}^{L-} \\
\left(g^{+}, g^{-}\right) \in \psi\left(\mathcal{P}_{L}^{c}\right)}} \mathbb{P}_{N, \gamma}\left(g^{+}, g^{-}\right) & =\mathbb{P}_{N, \gamma}\left(g^{+}, k_{L}\right) \sum_{\substack{g^{-} \in \mathcal{G}^{L-} \\
\left(g^{+}, g^{-}\right) \in \psi\left(\mathcal{P}_{L}^{c}\right)}}\left(\prod_{e \notin E\left(g^{-}\right):|e| \leq L}\left(\exp \left\{|e|^{\gamma}\right\}-1\right)\right) \\
& \leq \mathbb{P}_{N, \gamma}\left(g^{+}, k_{L}\right) \cdot \# \mathcal{G}^{L-} \cdot\left(\exp \left\{L^{\gamma+1}\right\}\right)^{N} \\
& \leq c^{N} \cdot \mathbb{P}_{N, \gamma}\left(g^{+}, k_{L}\right),
\end{aligned}
$$

where $c=c(L, \gamma)=2^{L} \cdot \exp \left\{L^{\gamma+1}\right\}$. Thus,

$$
\begin{aligned}
\mathbb{P}_{N, \gamma}^{b, \infty}\left(\mathcal{P}_{L}^{c}\right) & \leq \frac{1}{Z_{N, \gamma}^{b, \infty}} \sum_{g^{+} \in \mathcal{G}^{L+}} \exp \left\{-N^{b} \cdot\left(H_{L}^{*}\left(g^{+}, k_{L}\right)+1\right)\right\} \cdot c^{N} \cdot \mathbb{P}_{N, \gamma}\left(g^{+}, k_{L}\right) \\
& \leq c^{N} \cdot \exp \left\{-N^{b}\right\} \stackrel{N \rightarrow \infty}{\longrightarrow} 0
\end{aligned}
$$

as desired.

\subsection{Final remarks and open questions}

As mentioned after the statement of Theorem 41, the "transition window" for $b$ in case $\gamma>1$ is the interval $(0, \gamma)$ (regardless of $p$ ). Hence, if $p+1<\gamma$, Proposition 18 shows that there is no local limit even for some values of $b$ within the transition window.

For $\gamma>1$, this leaves open the cases:

$$
p=\infty, b \geq 0 \quad \text { and } \quad p \in[1, \infty), b \in[0, p+1] .
$$

We have no guess on whether or not local convergence occurs for some of these parameter values. 



\section{Bibliography}

[1] R. Ahlswede and D. E. Daykin, Inequalities for a pair of maps $S \times S \rightarrow S$ with $S$ a finite set, Math. Z. 165 (1979), 267-289. 11

[2] M. Aizenman, Translation invariance and instability of phase coexistence in the two dimensional Ising system, Communications in Mathematical Physics 73 (1980), no. 1, 83-94. 12

[3] M. Aizenman, J. T. Chayes, L. Chayes, and C. M. Newman, Discontinuity of the magnetization in one-dimensional $1 /|x-y|^{2}$ Ising and Potts models, Journal of Statistical Physics 50 (1988), no. 1, 1-40. 2, 16, 19

[4] M. Aizenman and C. M. Newman, Discontinuity of the percolation density in one-dimensional $1 /|x-y|^{2}$ percolation models, Comm. Math. Phys. 107 (1986), no. 4, 611-647. 2

[5] M. Aizenman and J. Wehr, Rounding of first-order phase transitions in systems with quenched disorder, Phys. Rev. Lett. 62 (1989), 2503-2506. 13

[6] M. Aizenman and J. Wehr, Rounding effects of quenched randomness on first-order phase transitions, Communications in Mathematical Physics 130 (1990), no. 3, 489-528. 13

[7] D. Alderson, J. Doyle, L. Li, and W. Willinger, A First-principles Approach to Understanding the Internet's Router-evel Topology, SIGCOMM Comput. Commun. Rev. 34 (2004), no. 4, 3-14. 3

[8] N. Alon, An extremal problem for sets with applications to graph theory, Journal of Combinatorial Theory, Series A 40 (1985), no. 1, 82-89. 59

[9] N. Alon, R. Bissacot, and E. O. Endo, Counting contours on trees, Letters in Mathematical Physics 107 (2017), no. 5, 887-899. 3

[10] R. G. Alves, A. Procacci, and R. Sanchis, Percolation on infinite graphs and isoperimetric inequalities, J. Stat. Phys. 149 (2012), no. 5, 831-845. 53, 54, 55

[11] E. Babson and I. Benjamini, Cut sets and normed cohomology with applications to percolation, Proc. Am. Math. Soc. 127 (1999), no. 2, 589-597. 3, 53, 55

[12] P. N. Balister and B. Bollobás, Counting regions with bounded surface area, Comm. Math. Phys. 273 (2007), no. 2, 305-315. 3, 53, 54, 63, 66, 67

[13] I. Benjamini, R. Lyons, and O. Schramm, Unimodular random trees, Ergodic Theory and Dynamical Systems 35 (2015), no. 2, 359-373. 74

[14] I. Benjamini and O. Schramm, Percolation Beyond $\mathbb{Z}^{d}$, Many Questions and a Few Answers, pp. 679-690, Springer New York, New York, NY, 2011. 54

[15] _ Recurrence of Distributional Limits of Finite Planar Graphs, pp. 533-545, Springer New York, New York, NY, 2011. 74, 75

[16] H. Berbee, Chains with infinite connections: Uniqueness and Markov representation, Probability Theory and Related Fields 76 (1987), no. 2, 243-253. 21, 22 
[17] N. Berger, C. Hoffman, and V. Sidoravicius, Non-uniqueness for specifications in $\ell^{2+\varepsilon}$, Ergodic Theory and Dynamical Systems (2017), 1-11. 21, 24

[18] S. Berghout, R. Fernández, and E. Verbitskiy, On the Relation between Gibbs and g-measures, Ergodic Theory and Dynamical Systems (2018), 1-26. 9, 21, 23, 24

[19] S. A. Bethuelsen and D. Conache, One-sided continuity properties for the Schonmann projection, ArXiv e-prints: 1802.02059 (2018). 24

[20] R. Bissacot, M. Cassandro, L. Cioletti, and E. Presutti, Phase Transitions in Ferromagnetic Ising Models with Spatially Dependent Magnetic Fields, Communications in Mathematical Physics 337 (2015), no. 1, 41-53. 3, 12, 14, 41, 42, 52

[21] R. Bissacot and L. Cioletti, Phase Transition in Ferromagnetic Ising Models with Non-uniform External Magnetic Fields, Journal of Statistical Physics 139 (2010), no. 5, 769-778. 12, 41, 42,69

[22] R. Bissacot, E. O. Endo, A. C. D. van Enter, B. Kimura, and W. M. Ruszel, Contour methods for Dyson models: weakening nearest-neighbor interactions and adding decaying fields, To appear in Annales Henri Poincaré (2018). 16, 39

[23] R. Bissacot, E. O. Endo, A. C. D. van Enter, and A. Le Ny, Entropic repulsion and lack of the g-measure property for Dyson models, ArXiv e-prints: 1705.03156 (2017). 3, 16

[24] R. Bissacot, E. O. Endo, and A. C. D. van Enter, Stability of the phase transition of criticalfield Ising model on Cayley trees under inhomogeneous external fields, Stochastic Processes and their Applications 127 (2017), no. 12, 4126-4138. 3

[25] P. M. Bleher, J. Ruiz, R. H. Schonmann, S. B. Shlosman, and V. A. Zagrebnov, Rigidity of the critical phases on a Cayley tree, Moscow Mathematical Journal 1 (2001), 345-363. 42

[26] T. Bodineau, Translation invariant Gibbs states for the Ising model, Probability Theory and Related Fields 135 (2006), no. 2, 153-168. 12

[27] B. Bollobás, On generalized graphs, Acta Mathematica Academiae Scientiarum Hungarica 16 (1965), no. 3, 447-452. 59

[28] A. Bovier, Statistical Mechanics of Disordered Systems: A Mathematical Perspective, Cambridge Series in Statistical and Probabilistic Mathematics (Book 18), Cambridge University Press; 1 edition (June 19, 2006), 2012. 7, 8, 53

[29] A. Bovier, I. Merola, E. Presutti, and M. Zahradník, On the Gibbs Phase Rule in the PirogovSinai Regime, Journal of Statistical Physics 114 (2004), no. 5, 1235-1267. 13

[30] R. Bowen, Equilibrium states and the ergodic theory of Anosov diffeomorphisms, 2 ed., vol. 470, Berlin; New York: Springer-Verlag, 2008. 9

[31] M. Bramson and S. Kalikow, Nonuniqueness in g-functions, Israel Journal of Mathematics 84 (1993), no. 1, 153-160. 21, 22, 24

[32] J. Bricmont and A. Kupiainen, Lower critical dimension for the random-field Ising model, Phys. Rev. Lett. 59 (1987), 1829-1832. 13

[33] _ Phase transition in the 3d random field Ising model, Communications in Mathematical Physics 116 (1988), no. 4, 539-572. 13

[34] J. Bricmont, J. L. Lebowitz, and C. E. Pfister, On the equivalence of boundary conditions, Journal of Statistical Physics 21 (1979), no. 5, 573-582. 31, 35 
[35] G. Brown and A. H. Dooley, Odometer actions on G-measures, Ergodic Theory and Dynamical Systems 11 (1991), no. 02, 279-307. 20, 21

[36] G. Brown and A. H. Dooley, On G-measures and product measures, Ergodic Theory and Dynamical Systems 18 (1998), no. 1, 95-107. 20, 21

[37] S. G. Brush, History of the Lenz-Ising Model, Rev. Mod. Phys. 39 (1967), 883-893. 2

[38] M. Campostrini and E. Vicari, Critical Behavior and Scaling in Trapped Systems, Phys. Rev. Lett. 102 (2009), 240601. 42

[39] M. Cassandro, P. A. Ferrari, I. Merola, and E. Presutti, Geometry of contours and Peierls estimates in $d=1$ Ising models with long range interactions, Journal of Mathematical Physics 46 (2005), no. 5, 053305. 2, 16, 19, 27, 29

[40] M. Cassandro, I. Merola, and P. Picco, Phase Separation for the Long Range One-dimensional Ising Model, Journal of Statistical Physics 167 (2017), no. 2, 351-382. 16, 27

[41] M. Cassandro, I. Merola, P. Picco, and U. Rozikov, One-Dimensional Ising Models with Long Range Interactions: Cluster Expansion, Phase-Separating Point, Communications in Mathematical Physics 327 (2014), no. 3, 951-991. 2, 16, 19, 27, 28, 29, 30, 34, 37

[42] M. Cassandro, E. Orlandi, and P. Picco, Phase Transition in the 1d Random Field Ising Model with Long Range Interaction, Communications in Mathematical Physics 288 (2009), no. 2, 731-744. 16, 19, 29

[43] _ Typical Gibbs Configurations for the 1d Random Field Ising Model with Long Range Interaction, Communications in Mathematical Physics 309 (2012), no. 1, 229-253. 16

[44] S. Chatterjee, On the Decay of Correlations in the Random Field Ising Model, To appear in Communications in Mathematical Physics, 2018. 13

[45] L. Cioletti and R. Vila, Graphical Representations for Ising and Potts Models in General External Fields, Journal of Statistical Physics 162 (2016), no. 1, 81-122. 3, 13, 52

[46] F. Comets, R. Fernández, and P. A. Ferrari, Processes with Long Memory: Regenerative Construction and Perfect Simulation, The Annals of Applied Probability 12 (2002), no. 3, 921-943. 22

[47] L. Coquille, Examples of DLR States Which are Not Weak Limits of Finite Volume Gibbs Measures with Deterministic Boundary Conditions, Journal of Statistical Physics 159 (2015), no. 4, 958-971. 9

[48] L. Coquille and Y. Velenik, A finite-volume version of Aizenman-Higuchi theorem for the 2d Ising model, Probability Theory and Related Fields 153 (2012), no. 1, 25-44. 12

[49] J. C. A. Dias and S. Friedli, Uniqueness vs. non-uniqueness for complete connections with modified majority rules, Probability Theory and Related Fields 164 (2016), no. 3, 893-929. 21,24

[50] R. L. Dobrushin, The Description of a Random Field by Means of Conditional Probabilities and Conditions of Its Regularity, Theory of Probability \& Its Applications 13 (1968), no. 2, 197-224. 9, 16

[51] R. L. Dobrushin, Analyticity of correlation functions in one-dimensional classical systems with slowly decreasing potentials, Comm. Math. Phys. 32 (1973), no. 4, 269-289. 16

[52] R. L. Dobrushin, Gibbs State Describing Coexistence of Phases for a Three-Dimensional Ising Model, Theory of Probability \& Its Applications 17 (1973), no. 4, 582-600. 12 
[53] Conditions for the absence of phase transitions in one-dimensional classical systems, Mathematics of the USSR-Sbornik 22 (1974), no. 1, 29-49. 16

[54] F. J. Dyson, Existence of a phase-transition in a one-dimensional Ising ferromagnet, Communications in Mathematical Physics 12 (1969), no. 2, 91-107. 2, 16, 19, 25

[55] _ An Ising ferromagnet with discontinuous long-range order, Communications in Mathematical Physics 21 (1971), no. 4, 269-283. 2, 19

[56] E. O. Endo and D. Valesin, Local limits of spatial Gibbs random graphs, ArXiv e-prints: 1712.03841 (2017). 3

[57] A. C. D. van Enter, V. N. Ermolaev, G. Iacobelli, and C. Külske, Gibbs-non-Gibbs properties for evolving Ising models on trees, Ann. Inst. H. Poincaré, Prob. Stat. 48 (2012), no. 3, 774791. 41

[58] A. C. D. van Enter, R. Fernández, and A. D. Sokal, Regularity properties and pathologies of position-space renormalization-group transformations: Scope and limitations of Gibbsian theory, Journal of Statistical Physics 72 (1993), no. 5, 879-1167. 8

[59] A. C. D. van Enter and A. Le Ny, Decimation of the Dyson-Ising ferromagnet, Stochastic Processes and their Applications 127 (2017), no. 11, 3776-3791. 8, 26, 27

[60] W. Evans, C. Kenyon, Y. Peres, and L. J. Schulman, Broadcasting on trees and the Ising model, Ann. Appl. Probab. 10 (2000), no. 2, 410-433. 43

[61] R. Fernández, Course 15 - Gibbsianness and Non-Gibbsianness in Lattice Random Fields, Mathematical Statistical Physics (A. Bovier, F. Dunlop, A. C. D. van Enter, F. den Hollander, and J. Dalibard, eds.), Les Houches, vol. 83, Elsevier, 2006, pp. 731-799. 7

[62] R. Fernández, S. Gallo, and G. Maillard, Regular g-measures are not always Gibbsian, Electron. Commun. Probab. 16 (2011), 732-740. 2, 3, 19, 23, 24

[63] R. Fernández and G. Maillard, Chains with Complete Connections and One-Dimensional Gibbs Measures, Electron. J. Probab. 9 (2004), 145-176. 19, 20, 23, 24

[64] _ Chains with Complete Connections: General Theory, Uniqueness, Loss of Memory and Mixing Properties, Journal of Statistical Physics 118 (2005), no. 3, 555-588. 20, 23, 24

[65] _ Construction of a Specification from its Singleton Part, ALEA (2006), no. 2, 297-315. 7

[66] R. Fernández and C.-E. Pfister, Global specifications and nonquasilocality of projections of Gibbs measures, Ann. Probab. 25 (1997), no. 3, 1284-1315. 25, 26, 27

[67] R. Fernández and A. Procacci, Cluster Expansion for Abstract Polymer Models. New Bounds from an Old Approach, Communications in Mathematical Physics 274 (2007), no. 1, 123-140. 66

[68] P. C. Fishburn and L. A. Shepp, The Ahlswede-Daykin Theorem, pp. 501-516, Springer US, Boston, MA, 2000. 11

[69] P. Flajolet and R. Sedgewick, Analytic combinatorics, Cambridge University Press, Cambridge, 2009. 56

[70] H. Föllmer, On the Global Markov Property, pp. 293-302, Springer Vienna, 1980. 25

[71] M. Formentin and C. Külske, On the purity of the free boundary condition Potts measure on random trees, Stochastic Processes and their Applications 119 (2009), no. 9, 2992-3005. 43 
[72] C. M. Fortuin, P. W. Kasteleyn, and J. Ginibre, Correlation inequalities on some partially ordered sets, Communications in Mathematical Physics 22 (1971), no. 2, 89-103. 9

[73] C. Fortuin and P. Kasteleyn, On the random-cluster model: I. Introduction and relation to other models, Physica 57 (1972), no. 4, 536 - 564. 13

[74] S. Friedli, A note on the Bramson-Kalikow process, Braz. J. Probab. Stat. 29 (2015), no. 2, 427-442. 21, 24

[75] S. Friedli and Y. Velenik, Statistical Mechanics of Lattice Systems: A Concrete Mathematical Introduction, Cambridge University Press, 2017. 7, 8, 10, 14

[76] J. Fröhlich, R. Israel, E. H. Lieb, and B. Simon, Phase transitions and reflection positivity. I. General theory and long range lattice models, Communications in Mathematical Physics 62 (1978), no. 1, 1-34. 16

[77] J. Fröhlich and T. Spencer, The phase transition in the one-dimensional Ising model with $1 / r^{2}$ interaction energy, Comm. Math. Phys. 84 (1982), no. 1, 87-101. 2, 16, 25, 27

[78] G. Gallavotti and S. Miracle-Sole, Statistical mechanics of lattice systems, Communications in Mathematical Physics 5 (1967), no. 5, 317-323. 16

[79] C. Gallesco, S. Gallo, and D. Y. Takahashi, Dynamic uniqueness for stochastic chains with unbounded memory, Stochastic Processes and their Applications 128 (2018), no. 2, 689-706. 21

[80] S. Gallo and F. Paccaut, On non-regular g-measures, Nonlinearity 26 (2013), no. 3, 763-776. 20,21

[81] A. Galves and E. Löcherbach, Stochastic chains with memory of variable length, Rissanen Festschrift (Grünwald et al. eds), TISCP Series 38 (2008), 117-133. 21

[82] D. Gandolfo, J. Ruiz, and S. Shlosman, A Manifold of Pure Gibbs States of the Ising Model on the Lobachevsky Plane, Communications in Mathematical Physics 334 (2015), no. 1, 313-330. 54

[83] N. Ganikhodjaev, Phase Transition in Ferromagnetic Lattice Models on a Cayley Tree with Non-uniform External Magnetic Fields, International Conference on Operations Research and Statistics Proceedings (2011), 106-110, Singapore Global Science and Technology Forum. 13, 42

[84] H.-O. Georgii, Gibbs measures and phase transitions; 2nd ed., De Gruyter Studies in Mathematics, De Gruyter, Berlin, 2011. 7, 8, 12, 24, 41, 43, 44, 45, 53, 65

[85] G. Giacomin, Random Polymer Models, Imperial College Press, London, 2007. 27

[86] S. Goldstein, A Note on Specifications, Z. Wahrsch. verw. Geb. 46 (1978), no. 1, 45-51. 7

[87] _ Remarks on the Global Markov Property, Comm. Math. Phys. 74 (1980), no. 3, 223234. 25

[88] M. González-Navarrete, E. Pechersky, and A. Yambartsev, Phase Transition in Ferromagnetic Ising Model with a Cell-Board External Field, Journal of Statistical Physics 162 (2016), no. 1, 139-161. 13

[89] R. B. Griffiths, Correlations in Ising Ferromagnets. I, Journal of Mathematical Physics 8 (1967), no. 3, 478-483. 11 
[90] - Correlations in Ising Ferromagnets. II. External Magnetic Fields, Journal of Mathematical Physics 8 (1967), no. 3, 484-489. 11

[91] G. R. Grimmett, The Random-Cluster Model, Grundlehren der mathematischen Wissenschaften, Springer-Verlag Berlin Heidelberg, 2006. 13

[92] T. E. Harris, On chains of infinite order, Pacific J. Math. 5 (1955), 707-724. 21, 22

[93] Y. Higuchi, On the absence of non-translation invariant Gibbs states for the two-dimensional Ising model, Random fields, Vol. I, II (Esztergom, 1979), Colloq. Math. Soc. János Bolyai, North-Holland, Amsterdam 27 (1981), no. 301, 517-534. 12

[94] W. Hoeffding, Probability inequalities for Sums of Bounded Random Variables, Journal of the American Statistical Association 58 (1963), no. 301, 13-30. 82

[95] R. Holley, Remarks on the FKG inequalities, Communications in Mathematical Physics 36 (1974), no. 3, 227-231. 11

[96] P. Hulse, On the Ergodic Properties of Gibbs States for Attractive Specifications, J. London Math. Soc. 2 (1991), no. 1, 119-124. 21, 25

[97] _ An example of non-unique g-measures, Ergodic Theory and Dynamical Systems 26 (2006), no. 2, 439-445. 21, 24

[98] J. Z. Imbrie and C. M. Newman, An intermediate phase with slow decay of correlations in one dimensional $\frac{1}{|x-y|^{2}}$ percolation, Ising and Potts models, Communications in Mathematical Physics 118 (1988), no. 2, 303-336. 19

[99] Y. Imry and S.-K. Ma, Random-Field Instability of the Ordered State of Continuous Symmetry, Physical Review Letters 35 (1975), 1399-1401. 13

[100] E. Ising, Beitrag zur Theorie des Ferromagnetismus, Zeitschrift fur Physik 31 (1925), 253-258. 2

[101] R. B. Israel, Convexity in the Theory of Lattice Gases, Princeton University Press, 1979. 8

[102] _ Some examples concerning the global Markov property, Comm. Math. Phys. 105 (1986), no. 4, 669-673. 25

[103] S. Janson, Large Deviations for Sums of Partly Dependent Random Variables, Random Struct. Algorithms 24 (2004), no. 3, 234-248. 82

[104] A. Johansson and A. Öberg, Square summability of variations of g-functions and uniqueness of g-measures, Mathematical Research Letters 10 (2003), no. 5, 587-601. 22

[105] A. Johansson, A. Öberg, and M. Pollicott, Unique Bernoulli g-measures, Journal of the European Mathematical Society 14 (2012), no. 5, 1599-1615. 21

[106] A. Johansson, A. Öberg, and M. Pollicott, Phase transitions in long-range Ising models and an optimal condition for factors of g-measures, Ergodic Theory and Dynamical Systems (2017), 1-14. 20,21

[107] K. Johansson, On separation of phases in one-dimensional gases, Communications in Mathematical Physics 169 (1995), no. 3, 521-561. 28

[108] J. Jonasson and J. E. Steif, Amenability and Phase Transition in the Ising Model, Journal of Theoretical Probability 12 (1999), no. 2, 549-559. 14, 41, 54

[109] M. Kac and C. J. Thompson, Critical Behavior of Several Lattice Models with Long-range Interaction, Journal of Mathematical Physics 10 (1969), no. 8, 1373-1386. 19 
[110] S. Kalikow, Random markov processes and uniform martingales, Israel Journal of Mathematics 71 (1990), no. 1, 33-54. 21

[111] S. Katsura and M. Takizawa, Bethe Lattice and the Bethe Approximation, Progress of Theoretical Physics 51 (1974), no. 1, 82-98. 41

[112] M. Keane, Strongly mixing g-measures, Inventiones mathematicae 16 (1972), no. 4, 309-324. $2,20,22$

[113] E. F. Keller, Revisiting "scale-free" networks, BioEssays 27 (2005), no. 10, 1060-1068. 3

[114] D. G. Kelly and S. Sherman, General Griffiths' Inequalities on Correlations in Ising Ferromagnets, Journal of Mathematical Physics 9 (1968), no. 3, 466-484. 11

[115] A. Kerimov, The one-dimensional long-range ferromagnetic Ising model with a periodic external field, Physica A: Statistical Mechanics and its Applications 391 (2012), no. 10, 2931-2935. 16

[116] D. Kerner and A. Némethi, A generalized FKG-inequality for compositions, Journal of Combinatorial Theory, Series A 146 (2017), 184-200. 11

[117] R. Kotecký and D. Preiss, Cluster expansion for abstract polymer models, Communications in Mathematical Physics 103 (1986), no. 3, 491-498. 66

[118] O. Kozlov, Gibbs Description of a System of Random Variables, Problems Inf. Transmission 10 (1974), no. 3, 258-265. 8, 23

[119] M. Krein and D. Milman, On extreme points of regular convex sets, Studia Mathematica 9 (1940), no. 1, 133-138. 9

[120] C. Kuelske and P. Schriever, Gradient Gibbs measures and fuzzy transformations on trees, ArXiv e-prints: 1609.00159 (2016). 41

[121] S. P. Lalley, Regenerative Representation for One-Dimensional Gibbs States, The Annals of Probability 14 (1986), no. 4, 1262-1271. 22

[122] O. E. Lanford and D. Ruelle, Observables at infinity and states with short range correlations in statistical mechanics, Communications in Mathematical Physics 13 (1969), no. 3, 194-215. 9

[123] J. L. Lebowitz and A. E. Mazel, Improved Peierls argument for high-dimensional Ising models, J. Statist. Phys. 90 (1998), no. 3-4, 1051-1059. 53, 63, 66

[124] F. Ledrappier, Principe variationnel et systèmes dynamiques symboliques, Z. Wahrscheinlichskeitstheorie Verw. Geb. 30 (1974), 185-202. 21

[125] T. D. Lee and C. N. Yang, Statistical Theory of Equations of State and Phase Transitions. II. Lattice Gas and Ising Model, Phys. Rev. 87 (1952), 410-419. 2, 11, 42

[126] J. Littin, Quasistationary Distributions when Infinity is an Entrance Boundary: Optimal Conditions for Phase Transition in One-Dimensional Ising Model by Peierls Argument and its Consequences, Ph.D. thesis, Marseille, 2013. 16

[127] J. Littin and P. Picco, Quasi-additive estimates on the Hamiltonian for the one-dimensional long range Ising model, Journal of Mathematical Physics 58 (2017), no. 7, 073301. 16, 39

[128] R. Lyons, The Ising model and percolation on trees and tree-like graphs, Communications in Mathematical Physics 125 (1989), no. 2, 337-353. 52 
[129] A. S. M. Iosifescu, On denumerable chains of infinite order, Z. Wahrscheinlichkeitstheorie verw. Gebiete 27 (1973), 195-214. 22

[130] C. Maes, F. Redig, A. V. Moffaert, and K. U. Leuven, Almost Gibbsian versus weakly Gibbsian measures, Stochastic Processes and their Applications 79 (1999), no. 1, 1-15. 26

[131] E. Mossel, Reconstruction on Trees: Beating the Second Eigenvalue, Ann. Appl. Probab. 11 (2001), no. 1, 285-300. 43

[132] J.-C. Mourrat and D. Valesin, Spatial Gibbs random graphs, Ann. Appl. Probab. 28 (2018), no. 2, 751-789. viii, $3,71,72,73,78,79$

[133] F. R. Nardi, E. Olivieri, and M. Zahradník, On the Ising Model with Strongly Anisotropic External Field, Journal of Statistical Physics 97 (1999), no. 1, 87-144. 13

[134] M. R. Palmer, W. Parry, and P. Walters, Large sets of endomorphisms and of $g$-measures, pp. 191-210, Springer Berlin Heidelberg, Berlin, Heidelberg, 1978. 21

[135] R. Peierls, On Ising's model of ferromagnetism, Mathematical Proceedings of the Cambridge Philosophical Society 32 (1936), no. 3, 477-481. 11, 42, 53, 63, 65

[136] C. E. Pfister, Large deviations and phase separation in the two-dimensional Ising model, Helv. Phys. Acta 64 (1991), 953-1054. 69

[137] C. E. Pfister and Y. Velenik, Mathematical theory of the wetting phenomenon in the 2D Ising model, Helv. Phys. Acta 69 (1996), no. 5-6, 949-973. 27

[138] C. Preston, Gibbs states on countable sets, Cambridge Tracts in Mathematics (Book 68), Cambridge University Press, London, 1974. 3, 41

[139] C. Preston, Construction of Specifications, Quantum Fields - Algebras, Processes (Vienna) (L. Streit, ed.), Springer Vienna, 1980, pp. 269-292. 7, 8

[140] C. J. Preston, A generalization of the FKG inequalities, Communications in Mathematical Physics 36 (1974), no. 3, 233-241. 9

[141] A. Raoufi, Translation-Invariant Gibbs States of Ising model: General Setting, ArXiv e-prints: 1710.07608 (2017). 12

[142] F. Rassoul-Agha and T. Seppäläinen, A course on large deviations with an introduction to Gibbs measures, Graduate Studies in Mathematics, vol. 162, American Mathematical Society, Providence, 2015. 53

[143] D. S. P. Richards, Algebraic methods toward higher-order probability inequalities, II, The Annals of Probability 32 (2004), no. 2, 1509-1544. 11

[144] Y. Rinott and M. Saks, Correlation inequalities and a conjecture for permanents, Combinatorica 13 (1993), no. 3, 269-277. 11

[145] J. Rissanen, A Universal Data Compression System, IEEE Trans. Inf. Theor. 29 (2006), no. 5, 656-664. 21

[146] U. A. Rozikov, Construction of an uncountable number of limiting Gibbs measures in the inhomogeneous Ising model, Theoretical and Mathematical Physics 118 (1999), no. 1, 77-84. 41

[147] U. A. Rozikov, On q-Component Models on Cayley Tree: Contour Method, Letters in Mathematical Physics 71 (2005), no. 1, 27-38. 53, 60, 67 
[148] _ An Example of One-Dimensional Phase Transition, Siberian Advances in Mathematics 16 (2006), no. 2, 121-125. 69

[149] _ A Contour Method on Cayley Trees, Journal of Statistical Physics 130 (2008), no. 4, 801-813. 53

[150] U. A. Rozikov, Gibbs Measures on Cayley Trees, World Scientific Publishing Company, 2013. $41,43,44,45,53$

[151] U. A. Rozikov, Gibbs Measures on Cayley Trees: Results and Open Problems, Reviews in Mathematical Physics 25 (2013), no. 01, 1330001. 53

[152] D. Ruelle, Statistical mechanics of a one-dimensional lattice gas, Communications in Mathematical Physics 9 (1968), no. 4, 267-278. 16

[153] D. Ruelle, On the use of "small external fields" in the problem of symmetry breakdown in statistical mechanics, Annals of Physics 69 (1972), no. 2, 364-374. 9, 42

[154] D. Ruelle, Statistical Mechanics: Rigorous Results, 2 ed., Imperial College Press, World Scientific Publishing, 1999. 53, 63

[155] _ Thermodynamic Formalism: The Mathematical Structure of Equilibrium Statistical Mechanics, 2 ed., Cambridge Mathematical Library, Cambridge University Press, 2004. 9

[156] _ The Mathematicia's Brain: A Personal Tour Through the Essentials of Mathematics and Some of the Great Minds Behind Them, Princeton University Press; First Edition edition (August 5, 2007), 2007. 2

[157] S. Sahi, The FKG Inequality for Partially Ordered Algebras, Journal of Theoretical Probability 21 (2008), no. 2, 449-458. 11

[158] A. N. Shiryaev, Probability (2Nd Ed.), Springer-Verlag New York, Inc., Secaucus, NJ, USA, 1995. 43

[159] Y. G. Sinai, Gibbs Measures in Ergodic Theory, Russian Mathematical Surveys 27 (1972), no. $4,21.9$

[160] S. Smirnov, Critical percolation in the plane: conformal invariance, Cardy's formula, scaling limits, Comptes Rendus de l'Académie des Sciences - Series I - Mathematics 333 (2001), no. 3, 239-244. 2

[161] A. D. Sokal, Existence of compatible families of proper regular conditional probabilities, Z. Wahrsch. verw. Geb. 56 (1981), no. 4, 537-548. 7

[162] O. Sporns and G. Tononi, Classes of network connectivity and dynamics, Complexity 7 (2001), no. $1,28-38.3$

[163] R. P. Stanley, Enumerative Combinatorics vol. 1, 2 ed., Cambridge Studies in Advanced Mathematics (Book 49), Cambridge University Press, 1997. 56

[164] _ Enumerative Combinatorics vol. 2, 2 ed., Cambridge Studies in Advanced Mathematics (Book 49), Cambridge University Press, 1999. 56

[165] Ö. Stenflo, Uniqueness in g-measures, Nonlinearity 16 (2003), no. 2, 403. 22

[166] W. G. Sullivan, Potentials for almost Markovian random fields, Comm. Math. Phys. 33 (1973), no. $1,61-74.8$

[167] E. Verbitskiy, On factors of g-measures, Indagationes Mathematicae 22 (2011), no. 3-4, 315329. 21 
[168] P. Walters, Ruelle's Operator Theorem and g-Measures, Transactions of the American Mathematical Society 214 (1975), 375-387. 21, 22

[169] H. von Weizsäcker, A simple example concerning the global Markov property of lattice random fields, Abstracta. 8th Winter School on Abstract Analysis, Czechoslovak Academy of Sciences, 1980, pp. 194-198. 25

[170] W. S. Yang and D. Klein, An "FKG equality" with applications to random environments, Statistics \& Probability Letters 46 (2000), no. 2, 203-209. 11 


\section{Curriculum Vitae}

\section{Personal informations:}

Full name: Eric Ossami Endo

Born: June $30^{\text {th }}, 1989$, São Paulo, Brazil

Nationality: Brazilian

Website: https://sites.google.com/view/ericendo/

\section{Education:}

2008-2011: Mathematic Bachelor: Institute of Mathematics and Statistics - University of São Paulo (IME-USP), Brazil.

2012-2014: Master in Mathematic. Advisor: Prof. Dr. Yoshiharu Kohayakawa, Institute of Mathematics and Statistics - University of São Paulo (IME-USP), Brazil. CNPq scholarship.

2014-2018: Ph. D. in Applied Mathematics. Advisors: Prof. Dr. Rodrigo Bissacot and Prof. Dr. Aernout C. D. van Enter. Coadvisor: Dr. Daniel Valesin. Institute of Mathematics and Statistics - University of São Paulo (IME-USP), Brazil; and Johann Bernoulli Institute for Mathematics and Computer Science - University of Groningen (RUG), the Netherlands. FAPESP grants: 2014/10637-9 and 2015/14434-8.

\section{Publications:}

1. N. Alon, R. Bissacot, and E.O. Endo. Counting Contours on Trees. Letters in Mathematical Physics 107, Issue 5: 887-899, 2017.

2. R. Bissacot, E.O. Endo, and A.C.D. van Enter. Stability of the Phase Transition of CriticalField Ising Model on Cayley Trees under Inhomogeneous External Fields. Stochastic Processes and their Applications 107, Issue 12: 4126-4138, 2017.

3. R. Bissacot, E.O. Endo, A.C.D. van Enter, B. Kimura and W. Ruszel. Contour methods for long-range Ising models: weakening nearest-neighbor interactions and adding decaying fields. Annales Henri Poincaré 19, Issue 8: 2557-2574, 2018.

\section{Accepted:}

1. R. Bissacot, E.O. Endo, A.C.D. van Enter, and A. Le Ny. Entropic repulsion and lack of the $g$-measure property for Dyson models. To appear in Communications in Mathematical Physics, 2018.

2. R. Bissacot, E.O. Endo, A.C.D. van Enter, B. Kimura, A. Le Ny, and W. Ruszel. Dyson models under renormalization and in weak fields. To appear in Sojourns in Probability and Statistical Physics, 2018.

\section{Submitted:}

1. E.O. Endo and D. Valesin. Local limits of spatial Gibbs random graphs. arXiv:1712.03841, 2017. 